\title{
THE SECOND COHOMOLOGY GROUPS OF NILPOTENT ORBITS IN CLASSICAL LIE ALGEBRAS
}

\author{
INDRANIL BISWAS, PRALAY CHATTERJEE, AND CHANDAN MAITY
}

\begin{abstract}
The second de Rham cohomology groups of nilpotent orbits in non-compact real forms of classical complex simple Lie algebras are explicitly computed. Furthermore, the first de Rham cohomology groups of nilpotent orbits in non-compact classical simple Lie algebras are computed; they are proven to be zero for nilpotent orbits in all the complex simple Lie algebras. A key component in these computations is a description of the second and first cohomology groups of homogeneous spaces of general connected Lie groups which is obtained here. This description, which generalizes a previous theorem of the first two authors, may be of independent interest.
\end{abstract}

\section{Contents}

1. Introduction 2

1.1. Context and motivations 2

1.2. Description of our results 2

1.3. Underlying complexities and our strategy 3

1.4. Organization of the paper 4

2. Notation and background $\quad 4$

2.1. $\epsilon-\sigma$ Hermitian forms and associated groups $\quad 5$

2.2. Some standard results associated to $\mathfrak{s l}_{2}(\mathbb{R})$-triples in semisimple Lie algebras $\quad 8$

2.3. Some notions associated to finite dimensional $\mathfrak{s l}_{2}(\mathbb{R})$-modules $\quad 8$

2.4. Partitions and (signed) Young diagrams 9

3. The second and first cohomologies of homogeneous spaces 11

4. Second cohomology groups of nilpotent orbits 17

4.1. Some preparatory results 17

4.2. Second cohomology groups of nilpotent orbits in $\mathfrak{s l}_{n}(\mathbb{R}) \quad 19$

4.3. Second cohomology groups of nilpotent orbits in $\mathfrak{s l}_{n}(\mathbb{H}) \quad 20$

4.4. Second cohomology groups of nilpotent orbits in $\mathfrak{s u}(p, q) \quad 21$

4.5. Second cohomology groups of nilpotent orbits in $\mathfrak{s o}(p, q) \quad 30$

4.6. Second cohomology groups of nilpotent orbits in $\mathfrak{s o}^{*}(2 n) \quad 40$

4.7. Second cohomology groups of nilpotent orbits in $\mathfrak{s p}(n, \mathbb{R}) \quad 47$

4.8. Second cohomology groups of nilpotent orbits in $\mathfrak{s p}(p, q) \quad 53$

5. First cohomology groups of nilpotent orbits 55

$\begin{array}{ll}\text { Appendix. Basic results on nilpotent orbits } & 57\end{array}$

2010 Mathematics Subject Classification. 57T15, $17 \mathrm{~B} 08$.

Key words and phrases. Nilpotent orbits, classical groups, homogeneous spaces. 


\section{INTRODUCTION}

1.1. Context and motivations. The nilpotent orbits in the semisimple Lie algebras, under the adjoint action of the associated semisimple Lie groups, form a rich class of homogeneous spaces which are extensively studied; see the book [CoMc] and the relatively recent article [Mc] for an account. Nevertheless, surprisingly for a very long period there seems to have been hardly any literature on the topological invariants of such orbits other than the description of fundamental groups in the case of simple Lie algebras. The computation of fundamental groups of such orbits is attributed to T. Springer, R. Steinberg [SpSt] for the classical case and A. Alexeevski [Al] for the complex exceptional case; see [CoMc, Cor 6.1.6, p. 91, pp. 128-134], [Mc, pp. 229-230]. We also refer to the works of D. King [Ki] and E. Sommers [So] in this regard.

It was only recently attention has been drawn to topological invariants of such orbits other than the fundamental groups. In [Ju], D. Juteau describes the integral cohomology of the minimal nontrivial nilpotent orbit in complex simple Lie algebras, and in [Cr], P. Crooks computed the equivariant rational cohomology algebras of the regular and minimal nilpotent orbits in complex simple Lie algebras under the action of a maximal (compact) torus. Contribution towards this by some of us began in [BC1] where the second cohomology groups of the nilpotent orbits in all the complex simple Lie algebras, under the adjoint action of the corresponding complex group, are computed; see [BC1, Theorems 5.4, 5.5, 5.6, 5.11, 5.12]. Recently in [ChMa] the second cohomology groups of nilpotent orbits are computed for most of the nilpotent orbits in non-compact non-complex exceptional Lie algebras, and for the rest of cases of nilpotent orbits, which are not covered in the above computations, upper bounds for the dimensions of the second cohomology groups are obtained; see [ChMa, Theorems 3.2-3.13].

Each orbit in a semisimple Lie algebra under the adjoint action is equipped with the KostantKirillov two form. In [BC1], besides finding invariants other than the fundamental groups, further motivation for initiating computation of the second cohomology groups naturally stem from the exactness criterion obtained in [BC1, Proposition 1.2] for the Kostant-Kirillov two-forms on adjoint orbits of arbitrary elements in real semisimple Lie groups with semisimple maximal compact subgroups. It should also be mentioned that [BC1, Proposition 1.2] generalises [ABB, Theorem 1.2] where the exactness criterion are obtained for the Kostant-Kirillov form on an adjoint orbit of a semisimple element in a complex semisimple Lie algebra.

1.2. Description of our results. As it is always the case with non-compact real forms of the complex simple Lie algebras, when the analogues questions as above are cast in this setting, because of their inherent complexities the computations become much more harder compared to the case of complex simple Lie algebras; see $\S 1.3$ for the precise description of the intricacies that occur in this case. In the present paper we carry forward what is initiated in [BC1], and using involved computations we describe the second and the first cohomology groups of all the nilpotent orbits in all the real classical Lie algebras which are non-complex and non-compact.

We first obtain a Lie theoretic formulation of the first and second cohomologies of nilpotent orbits in Theorem 4.2 when the underlying group is the connected component of the $\mathbb{R}$-points of a $\mathbb{R}$-simple algebraic group. Applying this result, and developing the computational machineries in $\S 4$ we next obtain the second cohomology groups of all the nilpotent orbits in Theorems $4.7,4.9,4.15,4.25,4.26,4.31,4.37,4.40$. Our computations, in particular, yield that the second cohomology groups vanish for all the nilpotent orbits in $\mathfrak{s l}_{n}(\mathbb{H})$, and for most of the nilpotent orbits in $\mathfrak{s l}_{n}(\mathbb{R})$. In the case of $\mathfrak{s u}(p, q)$ the second cohomology groups of the nilpotent orbits depend on their signed Young diagrams. It also follows that for the remaining cases of non-compact real forms of classical complex Lie algebras the second cohomology groups of the 
nilpotent orbits are related in a straightforward way to the partition component of the associated signed Young diagrams.

The methods involved above also steered us to describing the first cohomology groups in all the simple Lie algebras except the non-compact non-complex exceptional ones. Using the generalities in Theorem 4.2 it follows that the first cohomology groups of nilpotent orbits in noncompact simple Lie algebras are at the most one dimensional. However, more precise descriptions of the first cohomology groups of nilpotent orbits are obtained in Theorems 5.1, 5.2, 5.3, 5.4, $5.5,5.6,5.7,5.8$. In particular, we obtain that the first cohomology groups of nilpotent orbits vanish in the cases of all the complex simple Lie algebras and in $\mathfrak{s l}_{n}(\mathbb{H}), \mathfrak{s p}(p, q), \mathfrak{s l}_{n}(\mathbb{R})$ when $n \geq 3, \mathfrak{s o}(p, q)$ when $p \neq 2, q \neq 2$ and $(p, q) \neq(1,1)$. We also refer to [ChMa] for some results on the dimensions of the first cohomology groups of nilpotent orbits in non-compact non-complex exceptional Lie algebras.

The computations described above necessitate a description of the second cohomology groups of homogeneous spaces of Lie groups in full generality, which we formulate in Theorem 3.3, generalizing [BC1, Theorem 3.3]. We also obtain a description of the first cohomology groups in Theorem 3.6 in the same general set-up as above. In [BC1] to facilitate the computations in complex simple Lie algebras a suitable description of the second cohomology group of any homogeneous space of a connected Lie group was obtained in [BC1, Theorem 3.3] under the assumption that all the maximal compact subgroups of the Lie group are semisimple; see [BC2] for a relatively simple proof of [BC1, Theorem 3.3]. As only the complex simple Lie groups were considered in [BC1], this condition was not restrictive because the maximal compact subgroups in complex simple Lie groups are in fact simple Lie groups. However, in the present case of non-compact real forms of complex simple Lie algebras, the maximal compact subgroups of the associated simple real Lie groups are not necessarily semisimple, and hence [BC1, Theorem 3.3] can not be applied anymore, in general, to do the computations. Hence we need to invoke Theorem 3.3 and Theorem 3.6 where no additional conditions are imposed on the maximal compact subgroups therein. Although general theories of cohomology groups of (compact) homogeneous spaces are extensively studied in the past (see, for example [Bo1], [CE], [GHV]) we are unable to locate Theorem 3.3 and Theorem 3.6 in the literature and hence proofs are included for the sake of completeness. On the other hand, in view of their applicability the above results may be of independent interest as they are general and hold under a very mild restriction. We deduce stronger consequences of the above results in the special cases when the ambient Lie groups are complex semisimple or real simple; see Corollary 3.8 and Theorem 3.9.

1.3. Underlying complexities and our strategy. We now describe the underlying complexities that arise in the present setting of non-compact non-complex classical Lie algebras, and briefly sketch the strategy that is followed in this paper to overcome them.

In our setting without loss of generality the simple real Lie groups $G$ may as well be assumed to be the connected component of $\mathbb{R}$-points of $\mathbb{R}$-simple algebraic groups defined over $\mathbb{R}$. We set $\mathfrak{g}:=\operatorname{Lie} G$. In this setting the Lie theoretic formulation is obtained in Theorem 4.2; the computations in [ChMa] also use this result crucially. Let $X \in \mathfrak{g}$ be a non-zero nilpotent element, and $\mathcal{Z}_{G}(X)$ be the centralizer of $X$ in $G$. Let $M$ be a maximal compact subgroup in $G$, and $K$ be a maximal compact subgroup in $\mathcal{Z}_{G}(X)$ such that $K \subset M$. Let $\mathfrak{m}, \mathfrak{z}(\mathfrak{k})$ be the Lie algebras of $M$ and the center of $K$, respectively. Then Theorem 4.2 says that the computation of the second cohomology of the nilpotent orbits boils down to understanding the action of the component group $K / K^{\circ}$ on the subalgebra $\mathfrak{z}(\mathfrak{k}) \cap[\mathfrak{m}, \mathfrak{m}]$. Thus, when $M$ is semi-simple this amounts to describing the action of $K / K^{\circ}$ on $\mathfrak{z}(\mathfrak{k})$, and hence knowing the isomorphism class of $K$ is enough to compute the second cohomology groups in this case. However, when $M$ is not semisimple it is not enough to know the isomorphism classes of $K$ and $M$, rather one also needs to understand 
the embedding of $K$ in $M$; the case of $\mathfrak{s u}(p, q)$ as in Section 4.4 a typical example of such a situation. Although in the setting of non-compact classical groups the isomorphism classes of $M$ are well-known, and the isomorphism classes of $K$ can be obtained immediately either using Lemma 4.4 or by the work of Springer and Steinberg [SpSt], hardly anything can be concluded, from these isomorphism classes, on how $K$ is embedded in $M$. Thus, one needs to go above and beyond the computations done in [SpSt] to find out this embedding explicitly for all the nilpotent orbits in $\mathfrak{g}$, and major parts of the Sections 4.4, 4.5, 4.6 and 4.7 are devoted to do this.

1.4. Organization of the paper. The paper is organized as follows. In Section 2 some notation and terminologies are fixed, and some backgrounds needed in the paper are recalled. Section 3 deals with the second and first cohomology groups of general homogeneous spaces of connected Lie groups; Theorem 3.3 and Theorem 3.6 are proved, and some corollaries of them are drawn which are of independent interest. In Section 4 the second cohomology groups are computed in Theorems 4.7, 4.9, 4.15, 4.25, 4.26, 4.31, 4.37 and 4.40. In Section 5 the first cohomology groups are computed in Theorems 5.1, 5.2, 5.3, 5.4, 5.5, 5.6, 5.7 and 5.8. In the Appendix we workout certain details on nilpotent orbits that are used in Section 4.

The computations in the Appendix lead us to Remark 5.21 where we rectify an error in [CoMc, p. 139, Lemma 9.3.1] related to the parametrizations of nilpotent orbits.

\section{NotATiOn AND BACKGROUND}

In this section we fix some notation, and recall some backgrounds which will be used throughout in this paper. Subsequently, a few specialized notation are mentioned as and when they occur.

Once and for all fix a square root of -1 and call it $\sqrt{-1}$. The center of a group $G$ is denoted by $\mathcal{Z}(G)$ while the center of a Lie algebra $\mathfrak{g}$ is denoted by $\mathfrak{z}(\mathfrak{g})$. The Lie groups will be denoted by the capital letters, while the Lie algebra of a Lie group will be denoted by the corresponding lower case German letter, unless a different notation is explicitly mentioned. Sometimes, for notational convenience, the Lie algebra of a Lie group $G$ is also denoted by $\operatorname{Lie}(G)$. The connected component of $G$ containing the identity element is denoted by $G^{\circ}$. For a subgroup $H$ of $G$ and a subset $S$ of $\mathfrak{g}$, the subgroup of $H$ that fixes $S$ pointwise under the adjoint action is called the centralizer of $S$ in $H$; the centralizer of $S$ in $H$ is denoted by $\mathcal{Z}_{H}(S)$. Similarly, for a Lie subalgebra $\mathfrak{h} \subset \mathfrak{g}$ and a subset $S \subset \mathfrak{g}$, by $\mathfrak{z}_{\mathfrak{h}}(S)$ we will denote the subalgebra of $\mathfrak{h}$ consisting of all the elements that commute with every element of $S$.

Let $\Gamma$ be a group acting linearly on a vector space $V$. The subspace of $V$ fixed pointwise by the action of $\Gamma$ is denoted by $V^{\Gamma}$. If $G$ is a Lie group with Lie algebra $\mathfrak{g}$, then it is immediate that the adjoint (respectively, coadjoint) action of $G^{\circ}$ on $\mathfrak{z}(\mathfrak{g})$ (respectively, $\mathfrak{z}(\mathfrak{g})^{*}$ ) is trivial; in particular, one obtains a natural action of $G / G^{\circ}$ on $\mathfrak{z}(\mathfrak{g})$ (respectively, $\mathfrak{z}(\mathfrak{g})^{*}$ ). We denote by $[\mathfrak{z}(\mathfrak{g})]^{G / G^{\circ}}\left(\right.$ respectively, $\left[\mathfrak{z}(\mathfrak{g})^{*}\right]^{G / G^{\circ}}$ ) the space of points of $\mathfrak{z}(\mathfrak{g})$ (respectively, of $\left.\mathfrak{z}(\mathfrak{g})^{*}\right)$ fixed pointwise under the action of $G / G^{\circ}$.

For a real semisimple Lie group $G$, an element $X \in \mathfrak{g}$ is called nilpotent if the linear endomorphism

$$
\operatorname{ad}(X): \mathfrak{g} \longrightarrow \mathfrak{g}
$$

is nilpotent. The set of nilpotent elements in $\mathfrak{g}$ is denoted by $\mathcal{N}_{\mathfrak{g}}$. A nilpotent orbit is an orbit of a nilpotent element in $\mathfrak{g}$ under the adjoint action of $G$. The orbit of a nilpotent element $X \in \mathfrak{g}$ is denoted by $\mathcal{O}_{X}$. The set of all nilpotent orbits in $\mathfrak{g}$ under the adjoint action of $G$ is denoted by $\mathcal{N}(G)$. 
2.1. $\epsilon-\sigma$ Hermitian forms and associated groups. The notation $\mathbb{D}$ will stand for either $\mathbb{R}$ or $\mathbb{C}$ or $\mathbb{H}$, unless mentioned otherwise. Take a right vector space $V$ defined over $\mathbb{D}$. Let $\operatorname{End}_{\mathbb{D}}(V)$ be the right $\mathbb{R}$-algebra of $\mathbb{D}$-linear endomorphisms of $V$, and let $\mathrm{GL}(\mathrm{V})$ be the group of invertible elements of $\operatorname{End}_{\mathbb{D}}(V)$. For a $\mathbb{D}$-linear endomorphism $T \in \operatorname{End}_{\mathbb{D}}(V)$ and an ordered $\mathbb{D}$-basis $\mathcal{B}$ of $V$, the matrix of $T$ with respect to $\mathcal{B}$ is denoted by $[T]_{\mathcal{B}}$. Recall that if $\mathbb{D}=\mathbb{C}$ then $\operatorname{End}_{\mathbb{D}}(V)$ is also a (right) $\mathbb{C}$-algebra. When $\mathbb{D}$ is either $\mathbb{R}$ or $\mathbb{C}$, let

$$
\operatorname{tr}: \operatorname{End}_{\mathbb{D}}(V) \longrightarrow \mathbb{D} \text { and } \operatorname{det}: \operatorname{End}_{\mathbb{D}} V \longrightarrow \mathbb{D}
$$

respectively be the usual trace and determinant maps. Let $A$ be a central simple $\mathbb{R}$-algebra. Let

$$
\operatorname{Nrd}_{A}: A \longrightarrow \mathbb{R}
$$

be the reduced norm on $A$, and let $\operatorname{Trd}_{A}: A \longrightarrow \mathbb{R}$ be the reduced trace on $A$. When $\mathbb{D}=\mathbb{R}$ or $\mathbb{C}$, define

$$
\mathrm{SL}(V):=\{z \in \mathrm{GL}(V) \mid \operatorname{det}(z)=1\} \quad \text { and } \mathfrak{s l}(V):=\left\{y \in \operatorname{End}_{\mathbb{D}}(V) \mid \operatorname{tr}(y)=0\right\} .
$$

If $\mathbb{D}=\mathbb{H}$ then recall that $\operatorname{End}_{\mathbb{D}}(V)$ is a central simple $\mathbb{R}$-algebra. In that case, define

$$
\mathrm{SL}(V):=\left\{z \in \mathrm{GL}(V) \mid \operatorname{Nrd}_{\operatorname{End}_{\mathbb{D}} V}(z)=1\right\}
$$

and

$$
\mathfrak{s l}(V):=\left\{y \in \operatorname{End}_{\mathbb{D}}(V) \mid \operatorname{Trd}_{\operatorname{End}_{\mathbb{D}}(V)}(y)=0\right\} .
$$

Let $\mathbb{D}$ be $\mathbb{R}, \mathbb{C}$ or $\mathbb{H}$, as above. Let $\sigma$ be either the identity map Id or an involution of $\mathbb{D}$, meaning $\sigma$ is $\mathbb{R}$-linear with $\sigma^{2}=\mathrm{Id}$ and $\sigma(x y)=\sigma(y) \sigma(x)$ for all $x, y \in \mathbb{D}$. Let $\epsilon= \pm 1$. Following $[\mathrm{Bo} 2, \S 23.8$, p. 264] we call a map

$$
\langle\cdot, \cdot\rangle: V \times V \longrightarrow \mathbb{D}
$$

a $\epsilon-\sigma$ Hermitian form if

- $\langle\cdot, \cdot\rangle$ is additive in each argument,

- $\langle v, u\rangle=\epsilon \sigma(\langle u, v\rangle)$, and

- $\langle v \alpha, u\rangle=\sigma(\alpha)\langle v, u\rangle$ for all $v, u \in V$ and for all $\alpha \in \mathbb{D}$.

A $\epsilon-\sigma$ Hermitian form $\langle\cdot, \cdot\rangle$ is called non-degenerate if $\langle v, u\rangle=0$ for all $v$ if and only if $u=0$. All $\epsilon-\sigma$ Hermitian forms considered here will be assumed to be non-degenerate.

We define

$$
\mathrm{U}(V,\langle\cdot, \cdot\rangle):=\{T \in \mathrm{GL}(V) \mid\langle T v, T u\rangle=\langle v, u\rangle \forall v, u \in V\}
$$

and

$$
\mathfrak{u}(V,\langle\cdot, \cdot\rangle):=\left\{T \in \operatorname{End}_{\mathbb{D}}(V) \mid\langle T v, u\rangle+\langle v, T u\rangle=0 \forall v, u \in V\right\} .
$$

We next define

$$
\mathrm{SU}(V,\langle\cdot, \cdot\rangle):=\mathrm{U}(V,\langle\cdot, \cdot\rangle) \cap \mathrm{SL}(V) \quad \text { and } \quad \mathfrak{s u}(V,\langle\cdot, \cdot\rangle):=\mathfrak{u}(V,\langle\cdot, \cdot\rangle) \cap \mathfrak{s l}(V) .
$$

Recall that $\mathfrak{s u}(V,\langle\cdot, \cdot\rangle)$ is a simple Lie algebra (cf. [Kn, Chapter I, Section 8]).

If $\mathbb{D}=\mathbb{C}$, then multiplication by $\sqrt{-1}$ sends the non-degenerate $\epsilon-\sigma$ Hermitian forms on $V$ with $\epsilon=-1, \sigma \neq$ Id to the non-degenerate $\epsilon$ - $\sigma$ Hermitian forms with $\epsilon=1, \sigma \neq$ Id, and this mapping is a bijection. Hence, when $\mathbb{D}=\mathbb{C}$ and $\sigma \neq \mathrm{Id}$, we consider only the case where $\epsilon=1$. If $\mathbb{D}=\mathbb{H}$ and $\sigma=\mathrm{Id}$, then it can be easily seen that $\langle\cdot, \cdot\rangle \equiv 0$. As $\langle\cdot, \cdot\rangle$ is assumed to be non-degenerate, this, in particular, implies that there is no form $\langle\cdot, \cdot\rangle$ on $V$ with $\mathbb{D}=\mathbb{H}, \sigma=\mathrm{Id}$. 
We define the usual conjugations $\sigma_{c}$ on $\mathbb{C}$ by $\sigma_{c}\left(x_{1}+\sqrt{-1} x_{2}\right)=x_{1}-\sqrt{-1} x_{2}$, and on $\mathbb{H}$ by $\sigma_{c}\left(x_{1}+\mathbf{i} x_{2}+\mathbf{j} x_{3}+\mathbf{k} x_{4}\right)=x_{1}-\mathbf{i} x_{2}-\mathbf{j} x_{3}-\mathbf{k} x_{4}, x_{i} \in \mathbb{R}$ for $i=1, \cdots, 4$. Define $|z|:=\left(z \sigma_{c}(z)\right)^{1 / 2}$, for $z \in \mathbb{D}$. For $\alpha \in \mathbb{H}^{*}$ define

$$
C_{\alpha}: \mathbb{H} \longrightarrow \mathbb{H}, \quad x \longmapsto \alpha x \alpha^{-1}
$$

Clearly $C_{\alpha}$ is a $\mathbb{R}$-algebra automorphism, and $C_{\alpha}=C_{\alpha /|\alpha|}$. When $\mathbb{D}=\mathbb{H}$, the following facts justify that it is enough to consider the involution $\sigma_{c}$ instead of arbitrary involutions. The proof of the next lemma is a straightforward application of Skolem-Noether theorem which can be found in $[\mathrm{Bo} 2, \S 23.7$, p. 262].

Lemma 2.1 (cf. [Bo2, § 23.7, p. 262]). Let $\sigma: \mathbb{H} \longrightarrow \mathbb{H}$ be $\mathbb{R}$-linear with $\sigma(x y)=\sigma(y) \sigma(x)$ for all $x, y \in \mathbb{H}$. Then $\sigma$ is an involution, meaning $\sigma^{2}=\mathrm{Id}$, if and only if either $\sigma=\sigma_{c}$ or $\sigma=C_{\alpha} \circ \sigma_{c}$ for some $\alpha$ with $\alpha^{2}=-1$.

Lemma 2.2 (cf. [Bo2, §23.8, p. 264]). Let $\sigma: \mathbb{H} \longrightarrow \mathbb{H}$ be an involution, $\epsilon= \pm 1$ and

$$
\langle\cdot, \cdot\rangle: V \times V \longrightarrow \mathbb{D}
$$

$a \epsilon-\sigma$ Hermitian form. Let $\delta= \pm 1$ and $\alpha \in \mathbb{H}$ such that $|\alpha|=1$ and $\alpha \sigma(\alpha)^{-1}=\delta$. Then $\alpha\langle\cdot, \cdot\rangle$ is a $\delta \epsilon-C_{\alpha} \circ \sigma$ Hermitian form.

As a consequence of Lemma 2.2 if $\sigma: \mathbb{H} \longrightarrow \mathbb{H}$ is an involution, $\epsilon= \pm 1$ and

$$
\langle\cdot, \cdot\rangle: V \times V \longrightarrow \mathbb{D}
$$

a $\epsilon-\sigma$ Hermitian form, then $\alpha\langle\cdot, \cdot\rangle$ is a $\epsilon-\sigma_{c}$ Hermitian form with $\alpha \in \mathbb{H}$ being such that $\sigma=$ $C_{\alpha} \circ \sigma_{c}$ and $\alpha^{2}=-1$ (as in Lemma 2.1). In particular, an immediate consequence is that if $\sigma, \epsilon$ and $\langle\cdot, \cdot\rangle$ are as above, then there exists a $\epsilon-\sigma_{c}$ Hermitian form, say, $\langle\cdot, \cdot\rangle^{\prime}: V \times V \longrightarrow \mathbb{D}$ such that $\mathrm{SU}(V,\langle\cdot, \cdot\rangle)=\mathrm{SU}\left(V,\langle\cdot, \cdot\rangle^{\prime}\right)$ and $\mathfrak{s u}(V,\langle\cdot, \cdot\rangle)=\mathfrak{s u}\left(V,\langle\cdot, \cdot\rangle^{\prime}\right)$. In view of the above observations, without loss of generality, we may only consider the involution $\sigma_{c}$. From now on we will restrict to the involution $\sigma_{c}$ instead of arbitrary involutions on $\mathbb{D}$.

The case where $\mathbb{D}=\mathbb{C}, \sigma=\operatorname{Id}$ and $\epsilon= \pm 1$ is already investigated in [BC1]. Here the remaining three cases

(1) $\mathbb{D}=\mathbb{R}, \sigma=\operatorname{Id}$ and $\epsilon= \pm 1$,

(2) $\mathbb{D}=\mathbb{C}, \sigma=\sigma_{c}$ and $\epsilon=1$, and

$(3) \mathbb{D}=\mathbb{H}, \sigma=\sigma_{c}$ and $\epsilon= \pm 1$

will be investigated.

We next introduce certain standard nomenclature associated to the specific values of $\epsilon$ and $\sigma$. If $\sigma=\sigma_{c}$ and $\epsilon=1$, then $\langle\cdot, \cdot\rangle$ is called a Hermitian form. When $\sigma=\sigma_{c}$ and $\epsilon=-1$, then $\langle\cdot, \cdot\rangle$ is called a skew-Hermitian form. The form $\langle\cdot, \cdot\rangle$ is called symmetric if $\sigma=\operatorname{Id}$ and $\epsilon=1$. Lastly, if $\sigma=\operatorname{Id}$ and $\epsilon=-1$, then $\langle\cdot, \cdot\rangle$ is called a symplectic form. If $\langle\cdot, \cdot\rangle$ is a symmetric form on $V$, define

$$
\mathrm{SO}(V,\langle\cdot, \cdot\rangle):=\mathrm{SU}(V,\langle\cdot, \cdot\rangle) \text { and } \mathfrak{s o}(V,\langle\cdot, \cdot\rangle):=\mathfrak{s u}(V,\langle\cdot, \cdot\rangle) .
$$

Similarly, if $\langle\cdot, \cdot\rangle$ is a symplectic form on $V$, then define

$$
\operatorname{Sp}(V,\langle\cdot, \cdot\rangle):=\operatorname{SU}(V,\langle\cdot, \cdot\rangle) \text { and } \mathfrak{s p}(V,\langle\cdot, \cdot\rangle):=\mathfrak{s u}(V,\langle\cdot, \cdot\rangle) .
$$

When $\mathbb{D}=\mathbb{H}$ and $\langle\cdot, \cdot\rangle$ is a skew-Hermitian form on $V$, define

$$
\mathrm{SO}^{*}(V,\langle\cdot, \cdot\rangle):=\operatorname{SU}(V,\langle\cdot, \cdot\rangle) \text { and } \mathfrak{s o}^{*}(V,\langle\cdot, \cdot\rangle):=\mathfrak{s u}(V,\langle\cdot, \cdot\rangle) .
$$

As before, $V$ is a right vector space over $\mathbb{D}$. We now introduce some terminologies associated to certain types of $\mathbb{D}$-basis of $V$. When either $\mathbb{D}=\mathbb{R}, \sigma=\operatorname{Id}$ or $\mathbb{D}=\mathbb{C}, \sigma=\sigma_{c}$ or $\mathbb{D}=$ 
$\mathbb{H}, \sigma=\sigma_{c}$, for a $1-\sigma$ Hermitian form $\langle\cdot, \cdot\rangle$ on $V$, an orthogonal basis $\mathcal{A}$ of $V$ is called standard orthogonal if $\langle v, v\rangle= \pm 1$ for all $v \in \mathcal{A}$. For a standard orthogonal basis $\mathcal{A}$ of $V$, set

$$
p:=\#\{v \in \mathcal{A} \mid\langle v, v\rangle=1\} \quad \text { and } \quad q:=\#\{v \in \mathcal{A} \mid\langle v, v\rangle=-1\} .
$$

The pair $(p, q)$, which is independent of the choice of the standard orthogonal basis $\mathcal{A}$, is called the signature of $\langle\cdot, \cdot\rangle$. When $\mathbb{D}=\mathbb{C}$ and $\sigma=\sigma_{c}$, if $\langle\cdot, \cdot\rangle$ is a skew-Hermitian form on $V$ then $\sqrt{-1}\langle\cdot, \cdot\rangle$ is a Hermitian form on $V$; in this case the signature of $\langle\cdot, \cdot\rangle$ is defined to be the signature of the Hermitian form $\sqrt{-1}\langle\cdot, \cdot\rangle$.

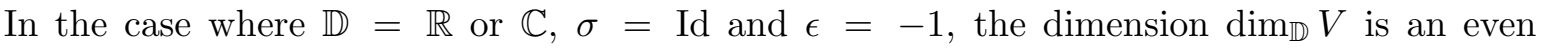
number. Let $2 n=\operatorname{dim}_{\mathbb{D}} V$. In this case an ordered basis $\mathcal{B}:=\left(v_{1}, \cdots, v_{n} ; v_{n+1}, \cdots, v_{2 n}\right)$ of $V$ is said to be symplectic if $\left\langle v_{i}, v_{n+i}\right\rangle=1$ for all $1 \leq i \leq n$ and $\left\langle v_{i}, v_{j}\right\rangle=0$ for all $j \neq n+i$. The ordered set $\left(v_{1}, \cdots, v_{n}\right)$ is called the positive part of $\mathcal{B}$ and it is denoted by $\mathcal{B}_{+}$. Similarly, the ordered set $\left(v_{n+1}, \cdots, v_{2 n}\right)$ is called the negative part of $\mathcal{B}$, and it is denoted by $\mathcal{B}_{-}$. The complex structure on $V$ associated to the above symplectic basis $\mathcal{B}$ is defined to be the $\mathbb{R}$-linear map

$$
J_{\mathcal{B}}: V \longrightarrow V, \quad v_{i} \longmapsto v_{n+i}, \quad v_{n+i} \longmapsto-v_{i} \forall 1 \leq i \leq n .
$$

If $\mathbb{D}=\mathbb{H}$ and $\langle\cdot, \cdot\rangle$ is a skew-Hermitian form on $V$, an orthogonal $\mathbb{H}$-basis

$$
\mathcal{B}:=\left(v_{1}, \cdots, v_{m}\right)
$$

of $V\left(m:=\operatorname{dim}_{\mathbb{H}} V\right)$ is said to be standard orthogonal if $\left\langle v_{r}, v_{r}\right\rangle=\mathbf{j}$ for all $1 \leq r \leq m$ and $\left\langle v_{r}, v_{s}\right\rangle=0$ for all $r \neq s$.

Take $P=\left(p_{i j}\right) \in \mathrm{M}_{r \times s}(\mathbb{D})$. Then $P^{t}$ denotes the transpose of $P$. If $\mathbb{D}=\mathbb{C}$ or $\mathbb{H}$, then define $\bar{P}:=\left(\sigma_{c}\left(p_{i j}\right)\right)$. Let

$$
\mathrm{I}_{p, q}:=\left(\begin{array}{ll}
\mathrm{I}_{p} & \\
& -\mathrm{I}_{q}
\end{array}\right), \quad \mathrm{J}_{n}:=\left(\begin{array}{cc} 
& -\mathrm{I}_{n} \\
\mathrm{I}_{n} &
\end{array}\right) .
$$

The classical groups and Lie algebras that we will be working with are:

$$
\begin{aligned}
& \mathrm{SL}_{n}(\mathbb{R}):=\left\{g \in \mathrm{GL}_{n}(\mathbb{R}) \mid \operatorname{det}(g)=1\right\}, \quad \mathfrak{s l}_{n}(\mathbb{R}):=\left\{z \in \mathrm{M}_{n}(\mathbb{R}) \mid \operatorname{tr}(z)=0\right\} ; \\
& \mathrm{SL}_{n}(\mathbb{H}):=\left\{g \in \mathrm{GL}_{n}(\mathbb{H}) \mid \operatorname{Nrd}_{\mathrm{M}_{n}(\mathbb{H})}(g)=1\right\}, \quad \mathfrak{s l}_{n}(\mathbb{H}):=\left\{z \in \mathrm{M}_{n}(\mathbb{H}) \mid \operatorname{Trd}_{\mathrm{M}_{n}(\mathbb{H})}(z)=0\right\} ; \\
& \mathrm{SU}(p, q):=\left\{g \in \mathrm{SL}_{p+q}(\mathbb{C}) \mid \bar{g}^{t} \mathrm{I}_{p, q} g=\mathrm{I}_{p, q}\right\}, \quad \mathfrak{s u}(p, q):=\left\{z \in \mathfrak{s l}_{p+q}(\mathbb{C}) \mid \bar{z}^{t} \mathrm{I}_{p, q}+\mathrm{I}_{p, q} z=0\right\} ; \\
& \mathrm{SO}(p, q):=\left\{g \in \mathrm{SL}_{p+q}(\mathbb{R}) \mid g^{t} \mathrm{I}_{p, q} g=\mathrm{I}_{p, q}\right\}, \quad \mathfrak{s o}(p, q):=\left\{z \in \mathfrak{s l}_{p+q}(\mathbb{R}) \mid z^{t} \mathrm{I}_{p, q}+\mathrm{I}_{p, q} z=0\right\} ; \\
& \mathrm{Sp}(p, q):=\left\{g \in \mathrm{SL}_{p+q}(\mathbb{H}) \mid \bar{g}^{t} \mathrm{I}_{p, q} g=\mathrm{I}_{p, q}\right\}, \quad \mathfrak{s p}(p, q):=\left\{z \in \mathfrak{s l}_{p+q}(\mathbb{H}) \mid \bar{z}^{t} \mathrm{I}_{p, q}+\mathrm{I}_{p, q} z=0\right\} ; \\
& \mathrm{Sp}(n, \mathbb{R}):=\left\{g \in \mathrm{SL}_{2 n}(\mathbb{R}) \mid g^{t} \mathrm{~J}_{n} g=\mathrm{J}_{n}\right\}, \quad \mathfrak{s p}(n, \mathbb{R}):=\left\{z \in \mathfrak{s l}_{2 n}(\mathbb{R}) \mid z^{t} \mathrm{~J}_{n}+\mathrm{J}_{n} z=0\right\} ; \\
& \mathrm{SO}^{*}(2 n):=\left\{g \in \mathrm{SL}_{n}(\mathbb{H}) \mid \bar{g}^{t} \mathbf{j} \mathrm{I}_{n} g=\mathbf{j I}_{n}\right\}, \quad \mathfrak{s o}^{*}(2 n):=\left\{z \in \mathfrak{s l}_{n}(\mathbb{H}) \mid \bar{z}^{t} \mathbf{j} \mathrm{I}_{n}+\mathrm{jI}_{n} z=0\right\} .
\end{aligned}
$$

For any group $H$, let $H_{\Delta}^{n}$ denote the diagonally embedded copy of $H$ in the $n$-fold direct product $H^{n}$. Let $V$ be a vector space over $\mathbb{D}$. Define $\mathfrak{d}_{V}: \operatorname{End}_{\mathbb{D}}(V) \longrightarrow \mathbb{D}^{*}$ to be $\mathfrak{d}_{V}:=$ det if $\mathbb{D}=\mathbb{C}$ or $\mathbb{R}$, and $\mathfrak{d}_{V}:=\operatorname{Nrd}_{\text {End }_{\mathbb{D}} V}$ if $\mathbb{D}=\mathbb{H}$. Let now $V_{i}, 1 \leq i \leq m$, be right vector spaces over $\mathbb{D}$. As before, $\mathbb{D}$ is either $\mathbb{R}$ or $\mathbb{C}$ or $\mathbb{H}$. For every $1 \leq i \leq m$, let $H_{i} \subset \operatorname{GL}\left(V_{i}\right)$ be a matrix subgroup. Now define the subgroup

$$
S\left(\prod_{i} H_{i}\right):=\left\{\left(h_{1}, \cdots, h_{m}\right) \in \prod_{i=1}^{m} H_{i} \mid \prod_{i} \mathfrak{d}_{V_{i}}\left(h_{i}\right)=1\right\} \subset \prod_{i=1}^{m} H_{i} .
$$

The following notation will allow us to write block-diagonal square matrices with many blocks in a convenient way. For $r$-many square matrices $A_{i} \in \mathrm{M}_{m_{i}}(\mathbb{D}), 1 \leq i \leq r$, the block diagonal square matrix of size $\sum m_{i} \times \sum m_{i}$, with $A_{i}$ as the $i$-th block in the diagonal, is denoted by 
$A_{1} \oplus \cdots \oplus A_{r}$. This is also abbreviated as $\oplus_{i=1}^{r} A_{i}$. Furthermore, if $B \in \mathrm{M}_{m}(\mathbb{D})$ and $s$ is a positive integer, then denote $B_{\mathbf{\Lambda}}^{s}:=\underbrace{B \oplus \cdots \oplus B}_{s \text {-many }}$.

The following lemma is a basic fact which readily follows from the Skolem-Noether theorem.

Lemma 2.3. Let $\alpha, \beta \in \mathbb{H}^{*}$ be such that $\operatorname{Re}(\alpha)=\operatorname{Re}(\beta)$ and $|\alpha|=|\beta|$. Then there exists an element $\lambda \in \mathbb{H}^{*}$ with $|\lambda|=1$ such that $\alpha=\lambda \beta \lambda^{-1}$.

2.2. Some standard results associated to $\mathfrak{s l}_{2}(\mathbb{R})$-triples in semisimple Lie algebras. For a Lie algebra $\mathfrak{g}$ over $\mathbb{R}$, a subset $\{X, H, Y\} \subset \mathfrak{g}$ is said to be a $\mathfrak{s l}_{2}(\mathbb{R})$-triple if $X \neq 0$, $[H, X]=2 X,[H, Y]=-2 Y$ and $[X, Y]=H$. It is immediate that $\operatorname{Span}_{\mathbb{R}}\{X, H, Y\}$ for a $\mathfrak{s l}_{2}(\mathbb{R})$-triple $\{X, H, Y\} \subset \mathfrak{g}$ is a $\mathbb{R}$-subalgebra of $\mathfrak{g}$ which is isomorphic to the Lie algebra $\mathfrak{s l}_{2}(\mathbb{R})$. We now recall a well-known result due to Jacobson and Morozov.

Theorem 2.4 (Jacobson-Morozov, cf. [CoMc, Theorem 9.2.1]). Let $X \in \mathfrak{g}$ be a non-zero nilpotent element in a real semisimple Lie algebra $\mathfrak{g}$. Then there exist $H, Y \in \mathfrak{g}$ such that $\{X, H, Y\} \subset \mathfrak{g}$ is a $\mathfrak{s l}_{2}(\mathbb{R})$-triple.

We now state a result relating two $\mathfrak{s l}_{2}(\mathbb{R})$-triples with common elements.

Lemma 2.5 (cf. [CoMc, Lemma 3.4.4]). Let $X$ be a nilpotent element, and let $H$ be a semisimple element, in a Lie algebra $\mathfrak{g}$ such that $\left\{X, H, Y_{1}\right\}$ and $\left\{X, H, Y_{2}\right\}$ are two $\mathfrak{s l}_{2}(\mathbb{R})$-triples in $\mathfrak{g}$. Then $Y_{1}=Y_{2}$.

We now record an immediate consequence of the above result.

Lemma 2.6. Let $\{X, H, Y\}$ be a $\mathfrak{s l}_{2}$-triple in the Lie algebra $\mathfrak{g}$ of a Lie group $G$. Then $\mathcal{Z}_{G}(X, H)=\mathcal{Z}_{G}(X, H, Y)$.

Proof. To prove the lemma it suffices to show that $\mathcal{Z}_{G}(X, H) \subset \mathcal{Z}_{G}(X, H, Y)$. Take any $g \in$ $\mathcal{Z}_{G}(X, H)$. Then $\{\operatorname{Ad}(g) X, \operatorname{Ad}(g) H, \operatorname{Ad}(g) Y\}=\{X, H, \operatorname{Ad}(g) Y\}$ is another $\mathfrak{s l}_{2}$-triple in $\mathfrak{g}$ containing $X$ and $H$. Using Lemma 2.5 we have $\operatorname{Ad}(g) Y=Y$, implying that $g \in \mathcal{Z}_{G}(X, H, Y)$.

2.3. Some notions associated to finite dimensional $\mathfrak{s l}_{2}(\mathbb{R})$-modules. Given an endomorphism $T \in \operatorname{End}_{\mathbb{R}}(W)$, where $W$ is a $\mathbb{R}$-vector space, and any $\lambda \in \mathbb{R}$, set

$$
W_{T, \lambda}:=\{w \in W \mid T w=w \lambda\} .
$$

Let $V$ be a right vector space of dimension $n$ over $\mathbb{D}$, where $\mathbb{D}$ is, as before, $\mathbb{R}$ or $\mathbb{C}$ or $\mathbb{H}$. Let $\{X, H, Y\} \subset \mathfrak{s l}(V)$ be a $\mathfrak{s l}_{2}(\mathbb{R})$-triple. Note that $V$ is also a $\mathbb{R}$-vector space using the inclusion $\mathbb{R} \hookrightarrow \mathbb{D}$. Hence $V$ is a module over $\operatorname{Span}_{\mathbb{R}}\{X, H, Y\} \simeq \mathfrak{s l}_{2}(\mathbb{R})$. For any positive integer $d$, let $M(d-1)$ denote the sum of all the $\mathbb{R}$-subspaces $A$ of $V$ such that

- $\operatorname{dim}_{\mathbb{R}} A=d$, and

- $A$ is an irreducible $\operatorname{Span}_{\mathbb{R}}\{X, H, Y\}$-submodules of $V$.

Then $M(d-1)$ is the isotypical component of $V$ containing all the irreducible submodules of $V$ with highest weight $d-1$. Let

$$
L(d-1):=V_{Y, 0} \cap M(d-1) .
$$

As the endomorphisms $X, H, Y$ of $V$ are $\mathbb{D}$-linear, the $\mathbb{R}$-subspaces $M(d-1), V_{Y, 0}$ and $L(d-1)$ of $V$ are also $\mathbb{D}$-subspaces. Let

$$
t_{d}:=\operatorname{dim}_{\mathbb{D}} L(d-1) .
$$


2.4. Partitions and (signed) Young diagrams. An ordered set of order $n$ is a $n$-tuple $\left(v_{1}, \cdots, v_{n}\right)$, where $v_{1}, \cdots, v_{n}$ are elements of some set, such that $v_{i} \neq v_{j}$ if $i \neq j$. If $w \in\left\{v_{1}, \cdots, v_{n}\right\}$, then write $w \in\left(v_{1}, \cdots, v_{n}\right)$. For two ordered sets $\left(v_{1}, \cdots, v_{n}\right)$ and $\left(w_{1}, \cdots, w_{m}\right)$, the ordered set $\left(v_{1}, \cdots, v_{n}, w_{1}, \cdots, w_{m}\right)$ will be denoted by

$$
\left(v_{1}, \cdots, v_{n}\right) \vee\left(w_{1}, \cdots, w_{m}\right) .
$$

Furthermore, for $k$-many ordered sets $\left(v_{1}^{i}, \cdots, v_{n_{i}}^{i}\right), 1 \leq i \leq k$, define the ordered set $\left(v_{1}^{1}, \cdots, v_{n_{1}}^{1}\right)$ $\vee \cdots \vee\left(v_{1}^{k}, \cdots, v_{n_{k}}^{k}\right)$ to be the following juxtaposition of ordered sets $\left(v_{1}^{i}, \cdots, v_{n_{i}}^{i}\right)$ with increasing $i$ :

$$
\left(v_{1}^{1}, \cdots, v_{n_{1}}^{1}\right) \vee \cdots \vee\left(v_{1}^{k}, \cdots, v_{n_{k}}^{k}\right):=\left(v_{1}^{1}, \cdots, v_{n_{1}}^{1}, \cdots, v_{1}^{k}, \cdots, v_{n_{k}}^{k}\right) .
$$

A partition of a positive integer $n$ is an object of the form $\left[d_{1}^{t_{1}}, \cdots, d_{s}^{t_{s}}\right]$, where $t_{i}, d_{i} \in \mathbb{N}$, $1 \leq i \leq s$, such that $\sum_{i=1}^{s} t_{i} d_{i}=n, t_{i} \geq 1$ and $d_{i+1}>d_{i}>0$ for all $i$; see [CoMc, $\S 3.1$, p. 30]. If $\left[d_{1}^{t_{1}}, \cdots, d_{s}^{t_{s}}\right]$ is a partition of $n$ in the above sense then $t_{i}$ is called the multiplicity of $d_{i}$. Henceforth, the multiplicity of $d_{i}$ will be denoted by $t_{d_{i}}$; this is to avoid any ambiguity. Let $\mathcal{P}(n)$ denote the set of all partitions of $n$. For a partition $\mathbf{d}=\left[d_{1}^{t_{d_{1}}}, \cdots, d_{s}^{t_{d_{s}}}\right]$ of $n$, define

$$
\mathbb{N}_{\mathbf{d}}:=\left\{d_{i} \mid 1 \leq i \leq s\right\}, \quad \mathbb{E}_{\mathbf{d}}:=\mathbb{N}_{\mathbf{d}} \cap(2 \mathbb{N}), \quad \mathbb{O}_{\mathbf{d}}:=\mathbb{N}_{\mathbf{d}} \backslash \mathbb{E}_{\mathbf{d}}
$$

Further define

$$
\mathbb{O}_{\mathbf{d}}^{1}:=\left\{d \mid d \in \mathbb{O}_{\mathbf{d}}, d \equiv 1 \quad(\bmod 4)\right\} \quad \text { and } \quad \mathbb{O}_{\mathbf{d}}^{3}:=\left\{d \mid d \in \mathbb{O}_{\mathbf{d}}, d \equiv 3 \quad(\bmod 4)\right\} .
$$

Following [CoMc, Theorem 9.3.3], a partition $\mathbf{d}$ of $n$ will be called even if $\mathbb{N}_{\mathbf{d}}=\mathbb{E}_{\mathbf{d}}$. Let $\mathcal{P}_{\text {even }}(n)$ be the subset of $\mathcal{P}(n)$ consisting of all even partitions of $n$. We call a partition $\mathbf{d}$ of $n$ to be very even if

- $\mathbf{d}$ is even, and

- $t_{\eta}$ is even for all $\eta \in \mathbb{N}_{\mathbf{d}}$.

Let $\mathcal{P}_{\mathrm{v} \text {.even }}(n)$ be the subset of $\mathcal{P}(n)$ consisting of all very even partitions of $n$. Now define

$$
\mathcal{P}_{1}(n):=\left\{\mathbf{d} \in \mathcal{P}(n) \mid t_{\eta} \text { is even for all } \eta \in \mathbb{E}_{\mathbf{d}}\right\}
$$

and

$$
\mathcal{P}_{-1}(n):=\left\{\mathbf{d} \in \mathcal{P}(n) \mid t_{\theta} \text { is even for all } \theta \in \mathbb{O}_{\mathbf{d}}\right\}
$$

Clearly, we have $\mathcal{P}_{\text {v.even }}(n) \subset \mathcal{P}_{1}(n)$.

Following [CoMc, p. 140] we define a Young diagram to be a left-justified array of rows of empty boxes arranged so that no row is shorter than the one below it; the size of a Young diagram is the number of empty boxes appearing in it. There is an obvious correspondence between the set of Young diagrams of size $n$ and the set $\mathcal{P}(n)$ of partitions of $n$. Hence the set of Young diagrams of size $n$ is also denoted by $\mathcal{P}(n)$. A signed Young diagram is a Young diagram in which every box is labeled with +1 or -1 such that the sign of 1 alternate across rows except when the length of the row is of the form $3(\bmod 4)$. In the latter case when the length of the row is of the form $3(\bmod 4)$ we will alternate the sign of 1 till the last but one and repeat the sign of 1 in the last box as in the last but one box; see Remark 5.21 why the choices of signs in this case deviate from that in the previous cases. The sign of 1 need not alternate down columns. Two signed Young diagrams are equivalent if and only if each can be obtained from the other by permuting rows of equal length. The signature of a signed Young diagram is the ordered pair of integers $(p, q)$ where $p$-many +1 and $q$-many -1 occur in it.

We next define certain sets using collections of matrices with entries comprising of signs \pm 1 , which are easily seen to be in bijection with sets of equivalence classes of various types of signed Young diagrams. These sets will be used in parametrizing the nilpotent orbits in the classical Lie algebras. 
For a partition $\mathbf{d} \in \mathcal{P}(n)$ and $d \in \mathbb{N}_{\mathbf{d}}$, we define the subset $\mathbf{A}_{d} \subset \mathrm{M}_{t_{d} \times d}(\mathbb{C})$ of matrices $\left(m_{i j}^{d}\right)$ with entries in the set $\{ \pm 1\}$ as follows :

$$
\mathbf{A}_{d}:=\left\{\left(m_{i j}^{d}\right) \in \mathrm{M}_{t_{d} \times d}(\mathbb{C}) \mid\left(m_{i j}^{d}\right) \text { satisfies (Yd.1) and }(\mathbf{Y d} .2)\right\} .
$$

Yd.1 There is an integer $0 \leq p_{d} \leq t_{d}$ such that

Yd.2

$$
m_{i 1}^{d}:= \begin{cases}+1 & \text { if } 1 \leq i \leq p_{d} \\ -1 & \text { if } p_{d}<i \leq t_{d}\end{cases}
$$

$$
\begin{aligned}
& m_{i j}^{d}:=(-1)^{j+1} m_{i 1}^{d} \quad \text { if } 1<j \leq d, d \in \mathbb{E}_{\mathbf{d}} \cup \mathbb{O}_{\mathbf{d}}^{1} ; \\
& m_{i j}^{d}:=\left\{\begin{array}{ll}
(-1)^{j+1} m_{i 1}^{d} & \text { if } 1<j \leq d-1 \\
-m_{i 1}^{d} & \text { if } j=d
\end{array}, d \in \mathbb{O}_{\mathbf{d}}^{3} .\right.
\end{aligned}
$$

For any $\left(m_{i j}^{d}\right) \in \mathbf{A}_{d}$ set

$$
\operatorname{sgn}_{+}\left(m_{i j}^{d}\right):=\#\left\{(i, j) \mid 1 \leq i \leq t_{d}, 1 \leq j \leq d, m_{i j}^{d}=+1\right\}
$$

and

$$
\operatorname{sgn}_{-}\left(m_{i j}^{d}\right):=\#\left\{(i, j) \mid 1 \leq i \leq t_{d}, 1 \leq j \leq d, m_{i j}^{d}=-1\right\} .
$$

Let $\mathcal{S}_{\mathbf{d}}(n):=\mathbf{A}_{d_{1}} \times \cdots \times \mathbf{A}_{d_{s}}$. For a pair of non-negative integers $(p, q)$ with $p+q=n$ we now define the subset $\mathcal{S}_{\mathbf{d}}(p, q) \subset \mathcal{S}_{\mathbf{d}}(n)$ by

$$
\mathcal{S}_{\mathbf{d}}(p, q):=\left\{\left(M_{d_{1}}, \ldots, M_{d_{s}}\right) \in \mathcal{S}_{\mathbf{d}}(n) \mid \sum_{i=1}^{s} \operatorname{sgn}_{+} M_{d_{i}}=p, \sum_{i=1}^{s} \operatorname{sgn}_{-} M_{d_{i}}=q\right\} .
$$

We also define

$$
\mathcal{Y}(p, q):=\left\{(\mathbf{d}, \mathbf{s g n}) \mid \mathbf{d} \in \mathcal{P}(n), \mathbf{s g n} \in \mathcal{S}_{\mathbf{d}}(p, q)\right\} .
$$

It is easy to see that there is a natural bijection between the set $\mathcal{Y}(p, q)$ and the equivalence classes of signed Young diagrams of size $p+q$ with signature $(p, q)$. Hence, we will call $\mathcal{Y}(p, q)$ the set of equivalence classes of signed Young diagrams of size $p+q$ with signature $(p, q)$.

For any $\mathbf{d} \in \mathcal{P}(n)$ and $d \in \mathbb{N}_{\mathbf{d}}$, define the subset $\mathbf{A}_{d, 1}$ of $\mathbf{A}_{d}$ by

$$
\mathbf{A}_{d, 1}:=\left\{\left(m_{i j}^{d}\right) \in \mathbf{A}_{d} \mid m_{i 1}^{d}=+1 \quad \forall 1 \leq i \leq t_{d}\right\} .
$$

Further define $\mathcal{S}_{\mathbf{d}}^{\text {even }}(p, q) \subset \mathcal{S}_{\mathbf{d}}(p, q)$ and $\mathcal{S}_{\mathbf{d}}^{\text {odd }}(n) \subset \mathcal{S}_{\mathbf{d}}(n)$ by

$$
\mathcal{S}_{\mathbf{d}}^{\text {even }}(p, q):=\left\{\left(M_{d_{1}}, \ldots, M_{d_{s}}\right) \in \mathcal{S}_{\mathbf{d}}(p, q) \mid M_{\eta} \in \mathbf{A}_{\eta, 1} \quad \forall \eta \in \mathbb{E}_{\mathbf{d}}\right\}
$$

and

$$
\mathcal{S}_{\mathbf{d}}^{\text {odd }}(n):=\left\{\left(M_{d_{1}}, \ldots, M_{d_{s}}\right) \in \mathcal{S}_{\mathbf{d}}(n) \mid M_{\theta} \in \mathbf{A}_{\theta, 1} \forall \theta \in \mathbb{O}_{\mathbf{d}}\right\} .
$$

For a pair $(p, q)$ of non-negative integers we define the sets $\mathcal{Y}^{\text {even }}(p, q)$ and $\mathcal{Y}_{1}^{\text {even }}(p, q)$ by

$$
\begin{aligned}
& \mathcal{Y}^{\text {even }}(p, q):=\left\{(\mathbf{d}, \mathbf{s g n}) \mid \mathbf{d} \in \mathcal{P}(n), \text { sgn } \in \mathcal{S}_{\mathbf{d}}^{\text {even }}(p, q)\right\}, \\
& \mathcal{Y}_{1}^{\text {even }}(p, q):=\left\{(\mathbf{d}, \mathbf{s g n}) \mid \mathbf{d} \in \mathcal{P}_{1}(n), \text { sgn } \in \mathcal{S}_{\mathbf{d}}^{\text {even }}(p, q)\right\} .
\end{aligned}
$$

Similarly, for a non-negative integer $n$, set

$$
\begin{gathered}
\mathcal{Y}^{\text {odd }}(n):=\left\{(\mathbf{d}, \mathbf{s g n}) \mid \mathbf{d} \in \mathcal{P}(n), \quad \text { sgn } \in \mathcal{S}_{\mathbf{d}}^{\text {odd }}(n)\right\}, \\
\mathcal{Y}_{-1}^{\text {odd }}(2 n):=\left\{(\mathbf{d}, \mathbf{s g n}) \mid \mathbf{d} \in \mathcal{P}_{-1}(2 n), \quad \text { sgn } \in \mathcal{S}_{\mathbf{d}}^{\text {odd }}(2 n)\right\} .
\end{gathered}
$$

Let $\mathbf{d} \in \mathcal{P}(n)$. For $\theta \in \mathbb{O}_{\mathbf{d}}$ and $M_{\theta}:=\left(m_{r s}^{\theta}\right) \in \mathbf{A}_{\theta}$, define

$$
l_{\theta, i}^{+}\left(M_{\theta}\right):=\#\left\{j \mid m_{i j}^{\theta}=+1\right\} \quad \text { and } \quad l_{\theta, i}^{-}\left(M_{\theta}\right):=\#\left\{j \mid m_{i j}^{\theta}=-1\right\}
$$


for all $1 \leq i \leq t_{\theta}$; set

$\mathcal{S}_{\mathbf{d}}^{\prime}(p, q):=\left\{\begin{array}{l|l}\left(M_{d_{1}}, \cdots, M_{d_{s}}\right) \in \mathcal{S}_{\mathbf{d}}^{\text {even }}(p, q) \mid \begin{array}{r}l_{\theta, i}^{+}\left(M_{\theta}\right) \text { is even for all } \theta \in \mathbb{O}_{\mathbf{d}}, 1 \leq i \leq t_{\theta} \\ \text { or } l_{\theta, i}^{-}\left(M_{\theta}\right) \text { is even for all } \theta \in \mathbb{O}_{\mathbf{d}}, 1 \leq i \leq t_{\theta}\end{array}\end{array}\right\}$.

\section{The SECOND AND FIRST COHOMOLOGIES OF HOMOGENEOUS SPACES}

In this section we obtain convenient descriptions of the second and the first cohomology groups of homogeneous spaces of general connected Lie groups with the purpose of applying them in the computations done in Sections 4 and 5. The main results are Theorems 3.3, 3.6, and they hold under the mild restriction that isotropy subgroups have finitely many connected components. Moreover, the results generalize those of $[\mathrm{BC} 1, \S 3]$ where it is assumed that the maximal compact subgroups of the ambient Lie groups are semisimple. We apply the above results to derive a stronger consequence in Theorem 3.9 where the ambient Lie group real simple.

Given a Lie algebra $\mathfrak{a}$ and an integer $n \geq 0$, let $\Omega^{n}(\mathfrak{a})$ denote the space of all $n$-forms on $\mathfrak{a}$. A $n$-form $\omega \in \Omega^{n}(\mathfrak{a})$ is said to annihilate a given subalgebra $\mathfrak{b} \subset \mathfrak{a}$ if $\omega\left(X_{1}, \cdots, X_{n}\right)=0$ whenever $X_{i} \in \mathfrak{b}$ for some $i$. Let $\Omega^{n}(\mathfrak{a} / \mathfrak{b})$ denote the space of $n$-forms on $\mathfrak{a}$ which annihilate $\mathfrak{b}$.

Let $L$ be a compact Lie group with Lie algebra l. Let $J \subset L$ be a closed subgroup with Lie algebra $\mathfrak{j} \subset \mathfrak{l}$. The space of $J$-invariant $p$-forms on $\mathfrak{l}$ will be denoted by $\Omega^{p}(\mathfrak{l})^{J}$. Note that $\omega \in \Omega^{p}(\mathfrak{l})^{J^{\circ}}$ if and only if

$$
\sum_{i=1}^{p} \omega\left(X_{1}, \cdots,\left[Y, X_{i}\right], \cdots, X_{p}\right)=0
$$

for all $Y \in \mathfrak{j}$ and all $\left(X_{1}, \cdots, X_{p}\right) \in \mathfrak{l}^{p}$. For a continuous function

$$
W: J \longrightarrow \Omega^{p}(\mathfrak{l})
$$

and a Haar measure $\mu_{J}$ on $J$, define the integral $\int_{J} W(g) d \mu_{J}(g) \in \Omega^{p}(\mathfrak{l})$ as follows:

$$
\left(\int_{J} W(g) d \mu_{J}(g)\right)\left(X_{1}, \cdots, X_{p}\right):=\int_{J} W(g)\left(X_{1}, \cdots, X_{p}\right) d \mu_{J}(g), \quad\left(X_{1}, \cdots, X_{p}\right) \in \mathfrak{l}^{p} .
$$

The above integral $\int_{J} W(g) d \mu_{J}(g)$ is also denoted by $\int_{J} W d \mu_{J}$. The following equations are straightforward.

$$
d \int_{J} W d \mu_{J}=\int_{J} d W d \mu_{J}
$$

For any $a \in L$,

$$
\operatorname{Ad}(a)^{*} \int_{J} W d \mu_{J}=\int_{J} \operatorname{Ad}(a)^{*} W d \mu_{J}
$$

For any $\omega \in \Omega^{p}(\mathfrak{l})$, from the left-invariance of the Haar measure $\mu_{J}$ on $J$ it follows that

$$
\int_{J}\left(\operatorname{Ad}(g)^{*} \omega\right) d \mu_{J}(g) \in \Omega^{p}(\mathfrak{l})^{J} .
$$

Lemma 3.1. Let $L$ be a compact Lie group with Lie algebra $\mathfrak{l}$. Let $p \geq 1$ be an integer.

(1) If $\omega \in \Omega^{p}(\mathfrak{l})$ is invariant then $d \omega=0$.

(2) Every element of $H^{p}(\mathfrak{l}, \mathbb{R})$ contains an unique invariant $\omega \in \Omega^{p}(\mathfrak{l})$.

(3) If $J \subset L$ is a closed subgroup, then

$$
\Omega^{p}(\mathfrak{l})^{J} \cap d\left(\Omega^{p-1}(\mathfrak{l})\right)=d\left(\Omega^{p-1}(\mathfrak{l})^{J}\right) .
$$

(4) If $L$ is connected and $\omega \in \Omega^{2}(\mathfrak{l})$, then $\omega \in \Omega^{2}(\mathfrak{l})^{L}$ if and only if $\omega \in \Omega^{2}(\mathfrak{l} /[\mathfrak{l}, \mathfrak{l}])$. 
Proof. Statement (1) is proved in [CE, p. 102, 12.3]. Statement (2) is proved in [CE, p. 102, Theorem 12.1].

To prove (3), note that it suffices to show that

$$
\Omega^{p}(\mathfrak{l})^{J} \cap d\left(\Omega^{p-1}(\mathfrak{l})\right) \subset d\left(\Omega^{p-1}(\mathfrak{l})^{J}\right) .
$$

Let $\mu_{J}$ denote the Haar measure on $J$ such that $\mu_{J}(J)=1$. For any $\omega \in \Omega^{p}(\mathfrak{l})^{J} \cap d\left(\Omega^{p-1}(\mathfrak{l})\right)$, we have $\omega=d \nu$ for some $\nu \in \Omega^{p-1}(\mathfrak{l})$. Now as $\omega$ is $J$-invariant, it follows that

$$
\omega=\operatorname{Ad}(g)^{*} d \nu=d \operatorname{Ad}(g)^{*} \nu
$$

for all $g \in J$. In particular, from (3.4) we have

$$
\omega=\int_{J}\left(d \operatorname{Ad}(g)^{*} \nu\right) d \mu_{J}(g)=d \int_{J}\left(\operatorname{Ad}(g)^{*} \nu\right) d \mu_{J}(g) .
$$

As $\mu_{J}$ is preserved by the left multiplication by elements of $J$, it now follows that

$$
\int_{J}\left(\operatorname{Ad}(g)^{*} \nu\right) d \mu_{J}(g) \in \Omega^{p-1}(\mathfrak{l})^{J} .
$$

This in turn implies that $\omega \in d\left(\Omega^{p-1}(\mathfrak{l})^{J}\right)$.

The proof of (4) is essentially contained in the proof of [Br, p. 309, Corollary 12.9]; we will give the details. Take any $\omega \in \Omega^{2}(\mathfrak{l})^{L}$. Lemma 3.1(1) says that $d \omega=0$. Thus, for all $x, y, z \in \mathfrak{l}$,

$$
d \omega(x, y, z)=-\omega([x, y], z)+\omega([x, z], y)-\omega([y, z], x)=0 .
$$

As $\omega$ is $L$-invariant, we also have

$$
-\omega([x, y], z)+\omega([x, z], y)=-(\omega([x, y], z)+\omega(y,[x, z])=0 .
$$

From (3.5) and (3.6) it follows that $\omega([y, z], x)=0$, therefore

$$
\omega([\mathfrak{l}, \mathfrak{l}], \mathfrak{l})=0 .
$$

This is equivalent to saying that $\omega \in \Omega^{2}(\mathfrak{l} /[\mathfrak{l}, \mathfrak{l}])$.

Conversely, if $\omega([\mathfrak{l}, \mathfrak{l}], \mathfrak{l})=0$, then it is immediate that $\omega$ satisfies $(3.1)$ for $p=2$. In particular, as $L$ is connected, we conclude that $\omega \in \Omega^{2}(\mathfrak{l})^{L}$. This completes the proof of (4).

Theorem 3.2 ([Mo]). Let $G$ be a connected Lie group, and let $H \subset G$ be a closed subgroup with finitely many connected components. Let $M$ be a maximal compact subgroup of $G$ such that $M \cap H$ is a maximal compact subgroup of $H$. Then the image of the natural embedding $M /(M \cap H) \hookrightarrow G / H$ is a deformation retraction of $G / H$.

Theorem 3.2 is proved in [Mo, p. 260, Theorem 3.1] under the assumption that $H$ is connected. However, as mentioned in [BC1], using [Ho, p. 180, Theorem 3.1], the proof as in [Mo] goes through when $H$ has finitely many connected components.

Let $G, H, M$ be as in Theorem 3.2, and let $K:=M \cap H$. As $M / K \hookrightarrow G / H$ is a deformation retraction by Theorem 3.2, we have

$$
H^{i}(G / H, \mathbb{R}) \simeq H^{i}(M / K, \mathbb{R}) \text { for all } i .
$$

Theorem 3.3. Let $G$ be a connected Lie group, and let $H \subset G$ be a closed subgroup with finitely many connected components. Let $K$ be a maximal compact subgroup of $H$, and let $M$ be a maximal compact subgroup of $G$ containing $K$. Then,

$$
H^{2}(G / H, \mathbb{R}) \simeq \Omega^{2}\left(\frac{\mathfrak{m}}{[\mathfrak{m}, \mathfrak{m}]+\mathfrak{k}}\right) \oplus\left[(\mathfrak{z}(\mathfrak{k}) \cap[\mathfrak{m}, \mathfrak{m}])^{*}\right]^{K / K^{\circ}}
$$


Proof. In view of (3.7) it is enough to show that

$$
H^{2}(M / K, \mathbb{R}) \simeq \Omega^{2}(\mathfrak{m} /([\mathfrak{m}, \mathfrak{m}]+\mathfrak{k})) \oplus\left[(\mathfrak{z}(\mathfrak{k}) \cap[\mathfrak{m}, \mathfrak{m}])^{*}\right]^{K / K^{\circ}} .
$$

As $M$ is compact and connected, from [Sp, p. 310, Theorem 30] and the formula given in [Sp, p. 313] we conclude that there are natural isomorphisms

$$
H^{i}(M / K, \mathbb{R}) \simeq \frac{\operatorname{Ker}\left(d: \Omega^{i}(\mathfrak{m} / \mathfrak{k})^{K} \rightarrow \Omega^{i+1}(\mathfrak{m} / \mathfrak{k})^{K}\right)}{d\left(\Omega^{i-1}(\mathfrak{m} / \mathfrak{k})^{K}\right)} \quad \forall i
$$

Setting $i=2$ in (3.9),

$$
H^{2}(M / K, \mathbb{R}) \simeq \frac{\operatorname{Ker}\left(d: \Omega^{2}(\mathfrak{m} / \mathfrak{k})^{K} \rightarrow \Omega^{3}(\mathfrak{m} / \mathfrak{k})^{K}\right)}{d\left(\Omega^{1}(\mathfrak{m} / \mathfrak{k})^{K}\right)} .
$$

The numerator and the denominator in (3.10) will be identified.

We claim that

$$
\operatorname{Ker}\left(d: \Omega^{2}(\mathfrak{m} / \mathfrak{k})^{K} \rightarrow \Omega^{3}(\mathfrak{m} / \mathfrak{k})^{K}\right)=\Omega^{2}(\mathfrak{m} / \mathfrak{k})^{M} \oplus d\left(\Omega^{1}(\mathfrak{m})^{K}\right) .
$$

To prove the claim, first note that $d\left(\Omega^{2}(\mathfrak{m} / \mathfrak{k})^{M}\right)=0$ by Lemma 3.1(1). Therefore, we have

$$
\Omega^{2}(\mathfrak{m} / \mathfrak{k})^{M}+d\left(\Omega^{1}(\mathfrak{m})^{K}\right) \subset \operatorname{Ker}\left(d: \Omega^{2}(\mathfrak{m} / \mathfrak{k})^{K} \rightarrow \Omega^{3}(\mathfrak{m} / \mathfrak{k})^{K}\right) .
$$

To prove the converse, take any $\omega \in \operatorname{Ker}\left(d: \Omega^{2}(\mathfrak{m} / \mathfrak{k})^{K} \rightarrow \Omega^{3}(\mathfrak{m} / \mathfrak{k})^{K}\right)$. Then by Lemma $3.1(2)$ there is an element $\widetilde{\omega} \in \Omega^{2}(\mathfrak{m})^{M}$ such that

$$
\omega-\widetilde{\omega} \in d\left(\Omega^{1}(\mathfrak{m})\right) .
$$

As $\omega \in \Omega^{2}(\mathfrak{m})^{K}$ and $\widetilde{\omega} \in \Omega^{2}(\mathfrak{m})^{M}$, it follows that $\omega-\widetilde{\omega} \in \Omega^{2}(\mathfrak{m})^{K}$. So (3.12) and Lemma $3.1(3)$ together imply that

$$
\omega-\widetilde{\omega} \in d\left(\Omega^{1}(\mathfrak{m})^{K}\right) .
$$

Take any $f \in \Omega^{1}(\mathfrak{m})^{K}$ such that $\omega-\widetilde{\omega}=d f$. As $f \in \Omega(\mathfrak{m})^{K}$, it follows that $d f \in \Omega^{2}(\mathfrak{m} / \mathfrak{k})^{K}$. Thus $\widetilde{\omega} \in \Omega^{2}(\mathfrak{m} / \mathfrak{k})^{M}$. This in turn implies that $\omega \in \Omega^{2}(\mathfrak{m} / \mathfrak{k})^{M}+d\left(\Omega^{1}(\mathfrak{m})^{K}\right)$. Therefore,

$$
\Omega^{2}(\mathfrak{m} / \mathfrak{k})^{M}+d\left(\Omega^{1}(\mathfrak{m})^{K}\right) \supset \operatorname{Ker}\left(d: \Omega^{2}(\mathfrak{m} / \mathfrak{k})^{K} \rightarrow \Omega^{3}(\mathfrak{m} / \mathfrak{k})^{K}\right) .
$$

To complete the proof of the claim, it now remains to show that

$$
\Omega^{2}(\mathfrak{m} / \mathfrak{k})^{M} \cap d\left(\Omega^{1}(\mathfrak{m})^{K}\right)=0 .
$$

To prove (3.13), take any $f_{1} \in \Omega^{1}(\mathfrak{m})^{K}$ such that $d f_{1} \in \Omega^{2}(\mathfrak{m} / \mathfrak{k})^{M}$. From Lemma 3.1(3) it follows that $d f_{1}=d f_{2}$ for some $f_{2} \in \Omega^{1}(\mathfrak{m})^{M}$. But then from Lemma 3.1(1) it follows that $d f_{2}=0$. Thus we have $d f_{1}=d f_{2}=0$. This proves (3.13), and the proof of the claim is complete.

Combining (3.10) and (3.11),

$$
H^{2}(M / K, \mathbb{R}) \simeq \Omega^{2}(\mathfrak{m} / \mathfrak{k})^{M} \oplus \frac{d\left(\Omega^{1}(\mathfrak{m})^{K}\right)}{d\left(\Omega^{1}(\mathfrak{m} / \mathfrak{k})^{K}\right)} .
$$

Moreover, as $M$ is connected, Lemma 3.1(4) implies that

$$
\Omega^{2}(\mathfrak{m} / \mathfrak{k})^{M} \simeq \Omega^{2}\left(\frac{\mathfrak{m}}{[\mathfrak{m}, \mathfrak{m}]+\mathfrak{k}}\right)
$$

We have

$$
\operatorname{Ker}\left(d: \Omega^{1}(\mathfrak{m}) \rightarrow \Omega^{2}(\mathfrak{m})\right)=\Omega^{1}(\mathfrak{m} /[\mathfrak{m}, \mathfrak{m}])
$$


In view of the above it is straightforward to check that

$$
\frac{d\left(\Omega^{1}(\mathfrak{m})^{K}\right)}{d\left(\Omega^{1}(\mathfrak{m} / \mathfrak{k})^{K}\right)} \simeq \frac{\Omega^{1}(\mathfrak{m})^{K}}{\Omega^{1}(\mathfrak{m} / \mathfrak{k})^{K}+\Omega^{1}(\mathfrak{m} /[\mathfrak{m}, \mathfrak{m}])^{K}} .
$$

We will identify the right-hand side of (3.16).

Consider the adjoint action of $K$ on $\mathfrak{m}$. As $K$ is compact, there is a $K$-invariant inner-product $\langle\cdot, \cdot\rangle$ on the $\mathbb{R}$-vector space $\mathfrak{m}$. Now decompose $\mathfrak{m}$ as follows.

$$
\begin{aligned}
\mathfrak{m} & =([\mathfrak{m}, \mathfrak{m}]+\mathfrak{k})+\mathfrak{z}(\mathfrak{m}) \\
& =([\mathfrak{m}, \mathfrak{m}]+\mathfrak{k}) \oplus\left((([\mathfrak{m}, \mathfrak{m}]+\mathfrak{k}) \cap \mathfrak{z}(\mathfrak{m}))^{\perp} \cap \mathfrak{z}(\mathfrak{m})\right) .
\end{aligned}
$$

We next decompose $[\mathfrak{m}, \mathfrak{m}]+\mathfrak{k}$ as

$$
[\mathfrak{m}, \mathfrak{m}]+\mathfrak{k}=\left(([\mathfrak{m}, \mathfrak{m}] \cap \mathfrak{k})^{\perp} \cap[\mathfrak{m}, \mathfrak{m}]\right) \oplus([\mathfrak{m}, \mathfrak{m}] \cap \mathfrak{k}) \oplus\left(([\mathfrak{m}, \mathfrak{m}] \cap \mathfrak{k})^{\perp} \cap \mathfrak{k}\right) .
$$

Using (3.17) and (3.18) the decomposition of $\mathfrak{m}$ is further refined as follows:

$$
\mathfrak{m}=([\mathfrak{m}, \mathfrak{m}]+\mathfrak{k}) \oplus\left((([\mathfrak{m}, \mathfrak{m}]+\mathfrak{k}) \cap \mathfrak{z}(\mathfrak{m}))^{\perp} \cap \mathfrak{z}(\mathfrak{m})\right)
$$

$=\left(([\mathfrak{m}, \mathfrak{m}] \cap \mathfrak{k})^{\perp} \cap[\mathfrak{m}, \mathfrak{m}]\right) \oplus([\mathfrak{m}, \mathfrak{m}] \cap \mathfrak{k}) \oplus\left(([\mathfrak{m}, \mathfrak{m}] \cap \mathfrak{k})^{\perp} \cap \mathfrak{k}\right) \oplus\left((([\mathfrak{m}, \mathfrak{m}]+\mathfrak{k}) \cap \mathfrak{z}(\mathfrak{m}))^{\perp} \cap \mathfrak{z}(\mathfrak{m})\right)$.

It is clear that all the direct summands in (3.19) are $K$-invariant. For notational convenience, set

Let

$$
\mathfrak{a}:=(([\mathfrak{m}, \mathfrak{m}]+\mathfrak{k}) \cap \mathfrak{z}(\mathfrak{m}))^{\perp} \quad \text { and } \quad \mathfrak{b}:=([\mathfrak{m}, \mathfrak{m}] \cap \mathfrak{k})^{\perp}
$$

$$
\left.\sigma: \Omega^{1}(\mathfrak{m})\right)=\mathfrak{m}^{*} \longrightarrow([\mathfrak{m}, \mathfrak{m}] \cap \mathfrak{b})^{*} \oplus([\mathfrak{m}, \mathfrak{m}] \cap \mathfrak{k})^{*} \oplus(\mathfrak{k} \cap \mathfrak{b})^{*} \oplus(\mathfrak{a} \cap \mathfrak{z}(\mathfrak{m}))^{*}
$$

be the isomorphism defined by

$$
f \longmapsto\left(\left.f\right|_{[\mathfrak{m}, \mathfrak{m}] \cap \mathfrak{b}},\left.f\right|_{[\mathfrak{m}, \mathfrak{m}] \cap \mathfrak{k}},\left.f\right|_{\mathfrak{k} \cap \mathfrak{b}},\left.f\right|_{\mathfrak{a} \cap \mathfrak{z}(\mathfrak{m})}\right) .
$$

As each of the subspaces of $\mathfrak{m}$ in (3.19) is $\operatorname{Ad}(K)$-invariant, the restriction of the isomorphism $\sigma$ in $(3.20)$ to $\Omega^{1}(\mathfrak{m})^{K}$ induces an isomorphism

$$
\tilde{\sigma}: \Omega^{1}(\mathfrak{m})^{K} \stackrel{\sim}{\longrightarrow}\left(([\mathfrak{m}, \mathfrak{m}] \cap \mathfrak{b})^{*}\right)^{K} \oplus\left(([\mathfrak{m}, \mathfrak{m}] \cap \mathfrak{k})^{*}\right)^{K} \oplus\left((\mathfrak{k} \cap \mathfrak{b})^{*}\right)^{K} \oplus\left((\mathfrak{a} \cap \mathfrak{z}(\mathfrak{m}))^{*}\right)^{K} .
$$

As $\mathfrak{k}=([\mathfrak{m}, \mathfrak{m}] \cap \mathfrak{k}) \oplus(\mathfrak{k} \cap \mathfrak{b})$ and $[\mathfrak{m}, \mathfrak{m}]=([\mathfrak{m}, \mathfrak{m}] \cap \mathfrak{k}) \oplus([\mathfrak{m}, \mathfrak{m}] \cap \mathfrak{b})$, it follows that

$$
\tilde{\sigma}\left(\Omega^{1}(\mathfrak{m} / \mathfrak{k})^{K}+\Omega^{1}(\mathfrak{m} /[\mathfrak{m}, \mathfrak{m}])^{K}\right)=\left(([\mathfrak{m}, \mathfrak{m}] \cap \mathfrak{b})^{*}\right)^{K} \oplus\left((\mathfrak{k} \cap \mathfrak{b})^{*}\right)^{K} \oplus\left((\mathfrak{a} \cap \mathfrak{z}(\mathfrak{m}))^{*}\right)^{K} .
$$

Thus from (3.21) and (3.22) it follows that

$$
\begin{aligned}
& \frac{\Omega^{1}(\mathfrak{m})^{K}}{\Omega^{1}(\mathfrak{m} / \mathfrak{k})^{K}+\Omega^{1}(\mathfrak{m} /[\mathfrak{m}, \mathfrak{m}])^{K}} \\
& \simeq \frac{\left(([\mathfrak{m}, \mathfrak{m}] \cap \mathfrak{b})^{*}\right)^{K} \oplus\left(([\mathfrak{m}, \mathfrak{m}] \cap \mathfrak{k})^{*}\right)^{K} \oplus\left((\mathfrak{k} \cap \mathfrak{b})^{*}\right)^{K} \oplus\left((\mathfrak{a} \cap \mathfrak{z}(\mathfrak{m}))^{*}\right)^{K}}{\left(([\mathfrak{m}, \mathfrak{m}] \cap \mathfrak{b})^{*}\right)^{K} \oplus\left((\mathfrak{k} \cap \mathfrak{b})^{*}\right)^{K} \oplus\left((\mathfrak{a} \cap \mathfrak{z}(\mathfrak{m}))^{*}\right)^{K}} \\
& \simeq\left(([\mathfrak{m}, \mathfrak{m}] \cap \mathfrak{k})^{*}\right)^{K} .
\end{aligned}
$$

As $[\mathfrak{m}, \mathfrak{m}] \cap \mathfrak{k}=[\mathfrak{k}, \mathfrak{k}] \oplus(\mathfrak{z}(\mathfrak{k}) \cap[\mathfrak{m}, \mathfrak{m}])$, it follows that

$$
\left(([\mathfrak{m}, \mathfrak{m}] \cap \mathfrak{k})^{*}\right)^{K} \simeq\left([\mathfrak{k}, \mathfrak{k}]^{*}\right)^{K} \oplus\left((\mathfrak{z}(\mathfrak{k}) \cap[\mathfrak{m}, \mathfrak{m}])^{*}\right)^{K} .
$$

In $[\mathrm{BC} 1, \S 3,(3.13)]$ it is proved that

$$
\left([\mathfrak{k}, \mathfrak{k}]^{*}\right)^{K}=0 .
$$

Thus from (3.23), (3.24) and (3.25) we have

$$
\frac{\Omega^{1}(\mathfrak{m})^{K}}{\Omega^{1}(\mathfrak{m} / \mathfrak{k})^{K}+\Omega^{1}(\mathfrak{m} /[\mathfrak{m}, \mathfrak{m}])^{K}} \simeq\left((\mathfrak{z}(\mathfrak{k}) \cap[\mathfrak{m}, \mathfrak{m}])^{*}\right)^{K} .
$$


Combining (3.16) and (3.26),

$$
\frac{d\left(\Omega^{1}(\mathfrak{m})^{K}\right)}{d\left(\Omega^{1}(\mathfrak{m} / \mathfrak{k})^{K}\right)} \simeq \frac{\Omega^{1}(\mathfrak{m})^{K}}{\Omega^{1}(\mathfrak{m} / \mathfrak{k})^{K}+\Omega^{1}(\mathfrak{m} /[\mathfrak{m}, \mathfrak{m}])^{K}} \simeq\left((\mathfrak{z}(\mathfrak{k}) \cap[\mathfrak{m}, \mathfrak{m}])^{*}\right)^{K}
$$

Moreover, as $K^{\circ}$ acts trivially on $\left((\mathfrak{z}(\mathfrak{k}) \cap[\mathfrak{m}, \mathfrak{m}])^{*}\right)$,

$$
\left((\mathfrak{z}(\mathfrak{k}) \cap[\mathfrak{m}, \mathfrak{m}])^{*}\right)^{K} \simeq\left((\mathfrak{z}(\mathfrak{k}) \cap[\mathfrak{m}, \mathfrak{m}])^{*}\right)^{K / K^{\circ}} .
$$

Combining (3.27) and (3.28),

$$
\frac{d\left(\Omega^{1}(\mathfrak{m})^{K}\right)}{d\left(\Omega^{1}(\mathfrak{m} / \mathfrak{k})^{K}\right)} \simeq\left((\mathfrak{z}(\mathfrak{k}) \cap[\mathfrak{m}, \mathfrak{m}])^{*}\right)^{K / K^{\circ}} .
$$

This and (3.15) together imply that the right-hand side of (3.14) coincides with the right-hand side of (3.8). This completes the proof of the theorem.

Corollary 3.4. Let $G, H, K$ and $M$ be as in Theorem 3.3. If $\operatorname{dim}_{\mathbb{R}} \mathfrak{z}(\mathfrak{m})=1$, then

$$
H^{2}(G / H, \mathbb{R}) \simeq\left[(\mathfrak{z}(\mathfrak{k}) \cap[\mathfrak{m}, \mathfrak{m}])^{*}\right]^{K / K^{\circ}} .
$$

Proof. As $M$ is a compact Lie group, we have $\mathfrak{m}=\mathfrak{z}(\mathfrak{m}) \oplus[\mathfrak{m}, \mathfrak{m}]$. Thus,

$$
\operatorname{dim}_{\mathbb{R}}(\mathfrak{m} /([\mathfrak{m}, \mathfrak{m}]+\mathfrak{k})) \leq 1 .
$$

Now the corollary follows from Theorem 3.3.

Corollary 3.5. Let $G, H, K$ and $M$ be as in Theorem 3.3. If $K$ is semisimple, then

$$
H^{2}(G / H, \mathbb{R}) \simeq \Omega^{2}\left(\frac{\mathfrak{m}}{[\mathfrak{m}, \mathfrak{m}]+\mathfrak{k}}\right) .
$$

Proof. As $K$ is semisimple, we have $\mathfrak{z}(\mathfrak{k})=0$, so it follows from Theorem 3.3.

Theorem 3.6. Let $G, H, K$ and $M$ be as in Theorem 3.3. Then

$$
H^{1}(G / H, \mathbb{R}) \simeq \Omega^{1}\left(\frac{\mathfrak{m}}{[\mathfrak{m}, \mathfrak{m}]+\mathfrak{k}}\right) .
$$

Proof. In view of $(3.7)$ it is enough to show that $H^{1}(M / K, \mathbb{R}) \simeq \Omega^{1}(\mathfrak{m} /([\mathfrak{m}, \mathfrak{m}]+\mathfrak{k}))$. As $M$ is compact and connected, from [Sp, p. 310, Theorem 30] and the formula given in [Sp, p. 313] it follows that there are natural isomorphisms

$$
H^{i}(M / K, \mathbb{R}) \simeq \frac{\operatorname{Ker}\left(d: \Omega^{i}(\mathfrak{m} / \mathfrak{k})^{K} \rightarrow \Omega^{i+1}(\mathfrak{m} / \mathfrak{k})^{K}\right)}{d\left(\Omega^{i-1}(\mathfrak{m} / \mathfrak{k})^{K}\right)} \quad \forall i
$$

Setting $i=1$ in (3.29),

$$
H^{1}(M / K, \mathbb{R}) \simeq \operatorname{Ker}\left(d: \Omega^{1}(\mathfrak{m} / \mathfrak{k})^{K} \rightarrow \Omega^{2}(\mathfrak{m} / \mathfrak{k})^{K}\right) .
$$

We claim that

$$
\operatorname{Ker}\left(d: \Omega^{1}(\mathfrak{m} / \mathfrak{k})^{K} \rightarrow \Omega^{2}(\mathfrak{m} / \mathfrak{k})^{K}\right)=\Omega^{1}(\mathfrak{m} / \mathfrak{k})^{M} .
$$

To prove (3.31), first note that $\Omega^{1}(\mathfrak{m} / \mathfrak{k})^{M} \subseteq \Omega^{1}(\mathfrak{m} / \mathfrak{k})^{K}$. From Lemma 3.1(1) it follows that $d \alpha=0$ for any $\alpha \in \Omega^{1}(\mathfrak{m} / \mathfrak{k})^{M}$. Thus

$$
\Omega^{1}(\mathfrak{m} / \mathfrak{k})^{M} \subseteq \operatorname{Ker}\left(d: \Omega^{1}(\mathfrak{m} / \mathfrak{k})^{K} \rightarrow \Omega^{2}(\mathfrak{m} / \mathfrak{k})^{K}\right) .
$$

To prove the other way inclusion, take any $\alpha \in \operatorname{Ker}\left(d: \Omega^{1}(\mathfrak{m} / \mathfrak{k})^{K} \rightarrow \Omega^{2}(\mathfrak{m} / \mathfrak{k})^{K}\right)$. Then $d \alpha=0$ which in turn implies that $\alpha([X, Y])=0$ for all $X, Y \in \mathfrak{m}$. As $M$ is connected, using (3.1) it follows that $\alpha$ is $M$-invariant. This proves the claim in (3.31). 
As $\Omega^{1}(\mathfrak{m})^{M} \simeq \Omega^{1}(\mathfrak{m} /[\mathfrak{m}, \mathfrak{m}])$, it follows that

$$
\Omega^{1}(\mathfrak{m} / \mathfrak{k})^{M} \simeq \Omega^{1}\left(\frac{\mathfrak{m}}{[\mathfrak{m}, \mathfrak{m}]+\mathfrak{k}}\right) .
$$

Combining (3.30), (3.31), (3.32) we have

$$
H^{1}(M / K, \mathbb{R}) \simeq \Omega^{1}\left(\frac{\mathfrak{m}}{[\mathfrak{m}, \mathfrak{m}]+\mathfrak{k}}\right) .
$$

As noted before, the theorem follows from it.

Corollary 3.7. Let $G, H, K$ and $M$ be as in Theorem 3.3. Moreover, if $M$ is semisimple, then

$$
\operatorname{dim}_{\mathbb{R}} H^{1}(G / H, \mathbb{R})=0 .
$$

Proof. As $M$ is semisimple, we have $\mathfrak{m}=[\mathfrak{m}, \mathfrak{m}]$, and hence the corollary follows from Theorem 3.6 .

Recall that any maximal compact subgroup of a complex semisimple Lie group is semisimple. The following corollary now follows form (3.7) and Corollary 3.7.

Corollary 3.8. Let $G$ be a connected complex semisimple Lie group, and let $H \subset G$ be a closed subgroup with finitely many connected components. Then

$$
\operatorname{dim}_{\mathbb{R}} H^{1}(G / H, \mathbb{R})=0 .
$$

In the special case where $G$ is a simple real Lie group, the following result is a stronger form of Theorem 3.3 and Theorem 3.6.

Theorem 3.9. Let $G$ be a connected simple real Lie group, and let $H \subset G$ be a closed subgroup with finitely many connected components. Let $K$ be a maximal compact subgroup of $H$ and $M$ a maximal compact subgroup of $G$ containing $K$. Then

$$
H^{2}(G / H, \mathbb{R}) \simeq\left[(\mathfrak{z}(\mathfrak{k}) \cap[\mathfrak{m}, \mathfrak{m}])^{*}\right]^{K / K^{\circ}}
$$

and

$$
\operatorname{dim}_{\mathbb{R}} H^{1}(G / H, \mathbb{R})= \begin{cases}1 & \text { if } \mathfrak{k}+[\mathfrak{m}, \mathfrak{m}] \varsubsetneqq \mathfrak{m} \\ 0 & \text { if } \mathfrak{k}+[\mathfrak{m}, \mathfrak{m}]=\mathfrak{m} .\end{cases}
$$

In particular, $\operatorname{dim}_{\mathbb{R}} H^{1}(G / H, \mathbb{R}) \leq 1$.

Proof. Since $M$ is a maximal compact subgroup of a real simple Lie group, it follows from [He, Proposition 6.2 , p. 382] that $\operatorname{dim}_{\mathbb{R}} \mathfrak{z}(\mathfrak{m})$ is either 0 or 1 . In both these cases we have $\Omega^{2}(\mathfrak{m} /([\mathfrak{m}, \mathfrak{m}]+\mathfrak{k}))=0$. In view of Theorem 3.3 and $(3.7)$, it follows that

$$
H^{2}(G / H, \mathbb{R}) \simeq\left[(\mathfrak{z}(\mathfrak{k}) \cap[\mathfrak{m}, \mathfrak{m}])^{*}\right]^{K / K^{\circ}} .
$$

As $G$ is simple, we have $\operatorname{dim}_{\mathbb{R}} \mathfrak{z}(\mathfrak{m}) \leq 1$. Thus, we have either

$$
\mathfrak{k}+[\mathfrak{m}, \mathfrak{m}] \varsubsetneqq \mathfrak{m} \quad \text { or } \quad \mathfrak{k}+[\mathfrak{m}, \mathfrak{m}]=\mathfrak{m} .
$$

Therefore, from Theorem 3.6 and (3.7) we conclude that

$$
\operatorname{dim}_{\mathbb{R}} H^{1}(G / H, \mathbb{R})= \begin{cases}1 & \text { if } \mathfrak{k}+[\mathfrak{m}, \mathfrak{m}] \varsubsetneqq \mathfrak{m} \\ 0 & \text { if } \mathfrak{k}+[\mathfrak{m}, \mathfrak{m}]=\mathfrak{m} .\end{cases}
$$




\section{Second COHOMOlogy groups of nilpotent orbits}

In this section we will compute the second de Rham cohomology groups of the nilpotent orbits in real classical simple Lie algebras. The case of complex simple Lie algebras was dealt in [BC1], so this case will not be considered here.

4.1. Some preparatory results. Here we prove Theorem 4.2 which is crucial to our computations of the second and first cohomology groups of the nilpotent orbits. We begin with the following lemma.

Lemma 4.1. Let $\mathbf{G}$ be a semisimple algebraic group defined over $\mathbb{R}$, and let $G:=\mathbf{G}(\mathbb{R})$. Let $\{X, H, Y\}$ be a $\mathfrak{s l}_{2}(\mathbb{R})$-triple in Lie $G$. Then $\mathcal{Z}_{\mathbf{G}}(X, H, Y)$ is a (reductive) Levi subgroup of $\mathcal{Z}_{\mathrm{G}}(X)$ which is defined over $\mathbb{R}$.

Proof. The nontrivial fact that the group $\mathcal{Z}_{\mathrm{G}}(X, H, Y)$ is a (reductive) Levi subgroup of $\mathcal{Z}_{\mathrm{G}}(X)$ is proved in [CoMc, p. 50, Lemma 3.7.3]. Since $X, H, Y \in \operatorname{Lie} G$, it is immediate that the group $\mathcal{Z}_{\mathbf{G}}(X, H, Y)$ is defined over $\mathbb{R}$.

As any connected adjoint simple real Lie group is always of the form $\mathbf{G}(\mathbb{R})^{\circ}$ for some ( $\mathbb{R}$ simple) algebraic group $\mathbf{G}$ defined over $\mathbb{R}$, it is enough to deal with nilpotent orbits in Lie $\mathbf{G}(\mathbb{R})$ under the adjoint action of $\mathbf{G}(\mathbb{R})^{\circ}$.

Theorem 4.2. Let $\mathbf{G}$ be an algebraic group defined over $\mathbb{R}$ such that $\mathbf{G}$ is $\mathbb{R}$-simple, and let $G:=\mathbf{G}(\mathbb{R})$. Let

$$
0 \neq X \in \operatorname{Lie} G
$$

be a nilpotent element and $\mathcal{O}_{X}$ be the orbit of $X$ under the adjoint action of the identity component $G^{\circ}$ on Lie $G$. Let $\{X, H, Y\}$ be a $\mathfrak{s l}_{2}(\mathbb{R})$-triple in Lie $G$. Let $K$ be a maximal compact subgroup in $\mathcal{Z}_{G^{\circ}}(X, H, Y)$ and $M$ a maximal compact subgroup of $G^{\circ}$ containing $K$. Then,

$$
H^{2}\left(\mathcal{O}_{X}, \mathbb{R}\right) \simeq\left[(\mathfrak{z}(\mathfrak{k}) \cap[\mathfrak{m}, \mathfrak{m}])^{*}\right]^{K / K^{\circ}}
$$

and

$$
\operatorname{dim}_{\mathbb{R}} H^{1}\left(\mathcal{O}_{X}, \mathbb{R}\right)= \begin{cases}1 & \text { if } \mathfrak{k}+[\mathfrak{m}, \mathfrak{m}] \varsubsetneqq \mathfrak{m} \\ 0 & \text { if } \mathfrak{k}+[\mathfrak{m}, \mathfrak{m}]=\mathfrak{m} .\end{cases}
$$

Proof. From Lemma 4.1 it follows that the group $\mathcal{Z}_{\mathbf{G}}(X, H, Y)$ is a (reductive) Levi subgroup of $\mathcal{Z}_{\mathrm{G}}(X)$. In particular, we have the semidirect product decomposition:

$$
\mathcal{Z}_{G^{\circ}}(X)=\mathcal{Z}_{G^{\circ}}(X, H, Y)\left(R_{u} \mathcal{Z}_{\mathbf{G}}(X)(\mathbb{R})\right),
$$

where $R_{u} \mathcal{Z}_{\mathrm{G}}(X)$ is the unipotent radical of $\mathcal{Z}_{\mathrm{G}}(X)$. As $R_{u} \mathcal{Z}_{\mathrm{G}}(X)(\mathbb{R})$ simply connected and nilpotent, this implies that any maximal compact subgroup in $\mathcal{Z}_{G^{\circ}}(X, H, Y)$ is a maximal compact subgroup in $\mathcal{Z}_{G^{\circ}}(X)$. Since $G^{\circ}$ is a connected simple real Lie group, the theorem now follows from Theorem 3.9.

Let $V$ be a right vector space of dimension $n$ over $\mathbb{D}$, where $\mathbb{D}$ is, as before, $\mathbb{R}$ or $\mathbb{C}$ or $\mathbb{H}$. Let $\{X, H, Y\} \subset \mathfrak{s l}(V)$ be a $\mathfrak{s l}_{2}(\mathbb{R})$-triple. We follow the notation established in Section 2.3. Consider the non-zero irreducible $\operatorname{Span}_{\mathbb{R}}\{X, H, Y\}$-submodules of $V$. Let $\left\{d_{1}, \cdots, d_{s}\right\}$, with $d_{1}<\cdots<d_{s}$, be the integers that occur as $\mathbb{R}$-dimension of $\operatorname{such}^{\operatorname{Span}_{\mathbb{R}}}\{X, H, Y\}$-modules. From Lemma 5.9(2) we have

Thus

$$
\sum_{i=1}^{s} t_{d_{i}} d_{i}=\operatorname{dim}_{\mathbb{D}} V=n
$$

$$
\mathbf{d}:=\left[d_{1}^{t_{d_{1}}}, \cdots, d_{s}^{t_{d_{s}}}\right] \in \mathcal{P}(n) .
$$


Consider $\mathbb{N}_{\mathbf{d}}, \mathbb{E}_{\mathbf{d}}$ and $\mathbb{O}_{\mathbf{d}}$ defined in Section 2.4. We have

$$
V=\bigoplus_{d \in \mathbb{N}_{\mathbf{d}}} M(d-1) \quad \text { and } \quad L(d-1)=V_{Y, 0} \cap V_{H, 1-d} \quad \text { for } \quad d \geq 1
$$

Let $\left(v_{1}^{d}, \cdots, v_{t_{d}}^{d}\right)$ be the ordered $\mathbb{D}$-basis of $L(d-1)$ as in Proposition 5.14 for $d \in \mathbb{N}_{\mathbf{d}}$. Then it follows from Proposition 5.10 and Proposition 5.14 that

$$
\mathcal{B}^{l}(d):=\left(X^{l} v_{1}^{d}, \cdots, X^{l} v_{t_{d}}^{d}\right)
$$

is an ordered $\mathbb{D}$-basis of $X^{l} L(d-1)$ for $0 \leq l \leq d-1$ with $d \in \mathbb{N}_{\mathbf{d}}$. Define

$$
\mathcal{B}(d):=\mathcal{B}^{0}(d) \vee \cdots \vee \mathcal{B}^{d-1}(d) \forall d \in \mathbb{N}_{\mathbf{d}} \text {, and } \mathcal{B}:=\mathcal{B}\left(d_{1}\right) \vee \cdots \vee \mathcal{B}\left(d_{s}\right) \text {. }
$$

Let

$$
\Lambda_{\mathcal{B}}: \operatorname{End}(V) \longrightarrow \mathrm{M}_{n}(\mathbb{D})
$$

be the isomorphism of $\mathbb{R}$-algebras with respect to the ordered basis $\mathcal{B}$. Next define the character

$$
\chi_{\mathbf{d}}: \prod_{d \in \mathbb{N}_{\mathbf{d}}} \operatorname{GL}(L(d-1)) \longrightarrow \mathbb{D}^{*}
$$

by

$$
\chi_{\mathbf{d}}\left(A_{t_{d_{1}}}, \cdots, A_{t_{d_{s}}}\right):= \begin{cases}\prod_{i=1}^{s}\left(\operatorname{det} A_{t_{d_{i}}}\right)^{d_{i}} & \text { if } \mathbb{D}=\mathbb{R} \text { or } \mathbb{C} \\ \prod_{i=1}^{s}\left(\operatorname{Nrd}_{\operatorname{End}_{\mathbb{H}}\left(L\left(d_{i}-1\right)\right)} A_{t_{d_{i}}}\right) d^{d_{i}} & \text { if } \mathbb{D}=\mathbb{H} .\end{cases}
$$

Henceforth, $\epsilon= \pm 1, \sigma: \mathbb{D} \longrightarrow \mathbb{D}$ will denote either the identity map or $\sigma_{c}$ (defined in Section 2.1) when $\mathbb{D}$ is $\mathbb{C}$ or $\mathbb{H}$. Let $\langle\cdot, \cdot\rangle: V \times V \longrightarrow \mathbb{D}$ be a $\epsilon-\sigma$ Hermitian form. Moreover, assume that $\{X, H, Y\}$ be a $\mathfrak{s l}_{2}(\mathbb{R})$-triple in $\mathfrak{s u}(V,\langle\cdot, \cdot\rangle)$. Define the form

$$
(\cdot, \cdot)_{d}: L(d-1) \times L(d-1) \longrightarrow \mathbb{D}, \quad(v, u)_{d}:=\left\langle v, X^{d-1} u\right\rangle
$$

as in [CoMc, p. 139].

Remark 4.3. In [CoMc, p.139], starting with a nilpotent element $X \in \mathfrak{s u}(V,\langle\cdot, \cdot\rangle)$, the form in (4.6) is defined on the highest weight space of $M(d-1)$ involving the element $Y$ of an $\mathfrak{s l}_{2}(\mathbb{R})$-triple $\{X, H, Y\}$. However, we work with a basis of $M(d-1)$ constructed using $X$ (see Proposition $5.14(2))$. Hence for our convenience the form in (4.6) is defined using $X$.

Lemma 4.4. Let $\mathrm{SL}(V)$ and $\mathrm{SU}(V,\langle\cdot, \cdot\rangle)$ be the groups as defined in Section 2.1.

(1) The following equality holds:

$$
\mathcal{Z}_{\mathrm{SL}(V)}(X, H, Y)=\left\{g \in \mathrm{SL}(V) \mid \begin{array}{c}
g\left(X^{l} L(d-1)\right) \subset X^{l} L(d-1), \\
{\left[\left.g\right|_{X^{l} L(d-1)}\right]_{\mathcal{B}^{l}(d)}=\left[\left.g\right|_{L(d-1)}\right]_{\mathcal{B}^{0}(d)} \text { for all } 0 \leq l<d, d \in \mathbb{N}_{\mathbf{d}}}
\end{array}\right\} .
$$

(2) In particular, $\mathcal{Z}_{\mathrm{SL}(V)}(X, H, Y) \simeq\left\{g \in \prod_{d \in \mathbb{N}_{\mathbf{d}}} \operatorname{GL}(L(d-1)) \mid \chi_{\mathbf{d}}(g)=1\right\}$.

(3) If $\{X, H, Y\}$ is a $\mathfrak{s l}_{2}(\mathbb{R})$-triple in $\mathfrak{s u}(V,\langle\cdot, \cdot\rangle)$, then

$$
\mathcal{Z}_{\mathrm{SU}(V,\langle\cdot, \cdot\rangle)}(X, H, Y)=\left\{\begin{array}{l|c}
g\left(X^{l} L(d-1)\right) \subset X^{l} L(d-1), \\
g \in \mathrm{SL}(V) \mid \begin{array}{c}
{\left[\left.g\right|_{X^{l} L(d-1)}\right]_{\mathcal{B}^{l}(d)}=\left[\left.g\right|_{L(d-1)}\right]_{\mathcal{B}^{0}(d)},(g x, g y)_{d}=(x, y)_{d}} \\
\text { for all } d \in \mathbb{N}_{\mathbf{d}}, 0 \leq l \leq d-1, \text { and } x, y \in L(d-1)
\end{array}
\end{array}\right\} ;
$$

here $(\cdot, \cdot)_{d}$ is the form on $L(d-1)$ defined in (4.6).

(4) In particular, $\mathcal{Z}_{\mathrm{SU}(V,\langle\cdot, \cdot\rangle)}(X, H, Y) \simeq\left\{g \in \prod_{d \in \mathbb{N}_{\mathbf{d}}} \mathrm{U}\left(L(d-1),(\cdot, \cdot)_{d}\right) \mid \chi_{\mathbf{d}}(g)=1\right\}$. 
Proof. For notational convenience, denote

$$
\mathcal{G}:=\left\{g \in \operatorname{SL}(V) \mid \begin{array}{c}
g\left(X^{l} L(d-1)\right) \subset X^{l} L(d-1) \\
{\left[\left.g\right|_{X^{l} L(d-1)}\right]_{\mathcal{B}^{l}(d)}=\left[\left.g\right|_{L(d-1)}\right]_{\mathcal{B}^{0}(d)} \forall 0 \leq l \leq d-1, d \in \mathbb{N}_{\mathbf{d}}}
\end{array}\right\} .
$$

Take any $g \in \mathcal{Z}_{\mathrm{SL}(V)}(X, H, Y)$. Then $g(L(d-1)) \subseteq L(d-1)$ by (4.2). In particular, it follows that $g\left(X^{l} L(d-1)\right) \subseteq X^{l} L(d-1)$ because $g$ commutes with $X$. Let $B_{d}:=\left[\left.g\right|_{L(d-1)}\right]_{\mathcal{B}^{0}(d)}$ for all $d \in \mathbb{N}_{\mathbf{d}}$. As $g$ commutes with $X$, it follows that $\left[\left.g\right|_{X^{l} L(d-1)}\right]_{\mathcal{B}^{l}(d)}=B_{d}$ for $0 \leq l \leq d-1$. This proves that $\mathcal{Z}_{\mathrm{SL}(V)}(X, H, Y) \subset \mathcal{G}$.

Take any $h \in \mathcal{G}$. Then $h\left(X^{l} L(d-1)\right) \subset X^{l} L(d-1)$ for all $0 \leq l \leq d-1$ and $d \in \mathbb{N}_{\mathbf{d}}$. For every $d \in \mathbb{N}_{\mathbf{d}}$, let $\left(a_{i j}^{d}\right)$ denote the matrix

$$
\left[\left.h\right|_{L(d-1)}\right]_{\mathcal{B}^{0}(d)} \in \mathrm{GL}_{t_{d}}(\mathbb{D}) .
$$

Then $\left(a_{i j}^{d}\right)=\left[\left.h\right|_{X^{l} L(d-1)}\right]_{\mathcal{B}^{l}(d)}$ for all $0 \leq l \leq d-1$.

We will show that $h$ commutes with $X$ and $H$. From (4.4) it follows that $\mathcal{B}$ is a $\mathbb{D}$-basis of $V$. Hence to prove that $X h=h X$ we need to show $X h\left(X^{l} v_{j}^{d}\right)=h X\left(X^{l} v_{j}^{d}\right)$ for all $1 \leq j \leq t_{d}$ and $0 \leq l \leq d-1$ with $d \in \mathbb{N}_{\mathbf{d}}$. However this follows from the following straightforward computation:

$$
h X\left(X^{l} v_{j}^{d}\right)=h X^{l+1} v_{j}^{d}=\sum_{i=1}^{t_{d}} X^{l+1} v_{i}^{d} a_{i j}^{d}=X\left(\sum_{i=1}^{t_{d}} X^{l} v_{i}^{d} a_{i j}^{d}\right)=X h\left(X^{l} v_{j}^{d}\right) .
$$

As $H$ acts as multiplication by a scalar in $\mathbb{R}$ (in fact, by a scalar in $\mathbb{Z}$ ) on the $\mathbb{D}$-basis $\mathcal{B}^{l}(d$ ) (of $X^{l} L(d-1)$ ) for all $0 \leq l \leq d-1$ with $d \in \mathbb{N}_{\mathbf{d}}$, it is immediate that $h$ commutes with $H$. In view of Lemma 2.6, we conclude that $h$ commutes with $Y$. This completes the proof of statement (1).

The third statement follows from statement (1) and Remark 5.15.

Remark 4.5. When $\mathbb{D}=\mathbb{R}$ or $\mathbb{C}$, the isomorphisms (2) and (4) in Lemma 4.4 were proved in [SpSt, p. 251, 1.8] and [SpSt, p. 261, 2.25] using only the Jordan canonical forms. However, as the non-commutativity of $\mathbb{H}$ creates technical difficulties in extending these results of [SpSt] to the case of $\mathbb{D}=\mathbb{H}$, we take a different approach by appealing to the Jacobson-Morozov theorem and the basic results on the structures of finite dimensional representations of $\mathfrak{s l}_{2}(\mathbb{R})$.

4.2. Second cohomology groups of nilpotent orbits in $\mathfrak{s l}_{n}(\mathbb{R})$. In this subsection we compute the second cohomology groups of nilpotent orbits in $\mathfrak{s l}_{n}(\mathbb{R})$ under the adjoint action of $\mathrm{SL}_{n}(\mathbb{R})$. We will follow notation as defined in $\S 2$. First recall a standard parametrization of $\mathcal{N}\left(\mathrm{SL}_{n}(\mathbb{R})\right)$, the set of all nilpotent orbits in $\mathfrak{s l}_{n}(\mathbb{R})$. Let $X \in \mathcal{N}_{\mathfrak{s l}_{n}(\mathbb{R})}$ be a nilpotent element in $\mathfrak{s l}_{n}(\mathbb{R})$ and $\mathcal{O}_{X}$ be the corresponding nilpotent orbit in $\mathfrak{s l}_{n}(\mathbb{R})$ under the adjoint action of $\mathrm{SL}_{n}(\mathbb{R})$. First assume that $X \neq 0$. Let $\{X, H, Y\} \subset \mathfrak{s l}_{n}(\mathbb{R})$ be a $\mathfrak{s l}_{2}(\mathbb{R})$-triple. Let $V:=\mathbb{R}^{n}$ be the right $\mathbb{R}$-vector space of column vectors. Let $\left\{d_{1}, \ldots, d_{s}\right\}$ with $d_{1}<\cdots<d_{s}$ be the finite set of natural numbers that occur as dimension of the non-zero irreducible $\operatorname{Span}_{\mathbb{R}}\{X, H, Y\}$-submodules of $V$. Recall that $M(d-1)$ is defined to be the isotypical component of $V$ containing all irreducible submodules of $V$ with highest weight $d-1$ and as in (2.2), we set $L(d-1):=V_{Y, 0} \cap M(d-1)$. Let $t_{d_{r}}:=\operatorname{dim}_{\mathbb{R}} L\left(d_{r}-1\right)$ for $1 \leq r \leq s$. Then $\left[d_{1}^{t_{d_{1}}}, \cdots, d_{s}^{t_{d_{s}}}\right] \in \mathcal{P}(n)$ because $\sum_{r=1}^{s} t_{d_{r}} d_{r}=n$. This induces a map $\Psi_{\mathrm{SL}_{n}(\mathbb{R})}: \mathcal{N}\left(\mathrm{SL}_{n}(\mathbb{R})\right) \longrightarrow \mathcal{P}(n)$. It is easy to see that $\Psi_{\mathrm{SL}_{n}(\mathbb{R})}\left(\mathcal{O}_{X}\right) \neq\left[1^{n}\right]$ as $X \neq 0$. By declaring $\Psi_{\mathrm{SL}_{n}(\mathbb{R})}\left(\mathcal{O}_{0}\right):=\left[1^{n}\right]$ we now have a surjective map

$$
\Psi_{\mathrm{SL}_{n}(\mathbb{R})}: \mathcal{N}\left(\mathrm{SL}_{n}(\mathbb{R})\right) \longrightarrow \mathcal{P}(n) \text {. }
$$

The following known result says that $\Psi_{\mathrm{SL}_{n}(\mathbb{R})}$ is "almost" a parametrization of the nilpotent orbits in $\mathfrak{s l}_{n}(\mathbb{R})$. 
Theorem 4.6 ([CoMc, Theorem 9.3.3]). For the map $\Psi_{\mathrm{SL}_{n}(\mathbb{R})}$ in (4.8),

$$
\# \Psi_{\mathrm{SL}_{n}(\mathbb{R})}^{-1}(\mathbf{d})= \begin{cases}1 & \text { for all } \mathbf{d} \in \mathcal{P}(n) \backslash \mathcal{P}_{\text {even }}(n) \\ 2 & \text { for all } \mathbf{d} \in \mathcal{P}_{\text {even }}(n) .\end{cases}
$$

Theorem 4.7. Let $X \in \mathfrak{s l}_{n}(\mathbb{R})$ be a nilpotent element. Let $\mathbf{d}=\left[d_{1}^{t_{d_{1}}}, \cdots, d_{s}^{t_{d_{s}}}\right] \in \mathcal{P}(n)$ be the partition associated to the orbit $\mathcal{O}_{X}$ (i.e., $\Psi_{\mathrm{SL}_{n}(\mathbb{R})}\left(\mathcal{O}_{X}\right)=\mathbf{d}$ in the notation of Theorem 4.6). Then the following hold:

(1) If $n \geq 3, \# \mathbb{O}_{\mathbf{d}}=1$ and $t_{\theta}=2$ for $\theta \in \mathbb{O}_{\mathbf{d}}$, then $\operatorname{dim}_{\mathbb{R}} H^{2}\left(\mathcal{O}_{X}, \mathbb{R}\right)=1$.

(2) In all the other cases $\operatorname{dim}_{\mathbb{R}} H^{2}\left(\mathcal{O}_{X}, \mathbb{R}\right)=0$.

Proof. This is obvious when $X=0$, so assume that $X \neq 0$.

The notation in Lemma 4.4 and the paragraph preceding it will be employed. Let $\{X, H, Y\}$ $\subset \mathfrak{s l}_{n}(\mathbb{R})$ be a $\mathfrak{s l}_{2}(\mathbb{R})$-triple. Let $K$ be a maximal compact subgroup of $\mathcal{Z}_{\mathrm{SL}_{n}(\mathbb{R})}(X, H, Y)$. Let $M$ be a maximal compact subgroup of $\mathrm{SL}_{n}(\mathbb{R})$ containing $K$. As $M \simeq \mathrm{SO}_{n}$, it follows that $\mathfrak{z}(\mathfrak{k}) \cap[\mathfrak{m}, \mathfrak{m}]=0$ when $n=2$, and $[\mathfrak{m}, \mathfrak{m}]=\mathfrak{m}$ when $n \geq 3$. Thus using Theorem 4.2 ,

$$
H^{2}\left(\mathcal{O}_{X}, \mathbb{R}\right) \simeq \begin{cases}0 & \text { if } n=2 \\ {\left[\mathfrak{z}(\mathfrak{k})^{*}\right]^{K / K^{\circ}}} & \text { if } n \geq 3 .\end{cases}
$$

Treating $\mathbb{R}^{n}$ as a $\operatorname{Span}_{\mathbb{R}}\{X, H, Y\}$-module through the standard action of $\mathfrak{s l}_{n}(\mathbb{R})$, construct a $\mathbb{R}$-basis $\mathcal{B}$ as in (4.4), and consider the $\mathbb{R}$-algebra isomorphism $\Lambda_{\mathcal{B}}$ in (4.5). It now follows from Lemma 4.4(2) that the restriction of $\Lambda_{\mathcal{B}}$ induces an isomorphism of Lie groups:

$$
\Lambda_{\mathcal{B}}: \mathcal{Z}_{\mathrm{SL}_{n}(\mathbb{R})}(X, H, Y) \stackrel{\sim}{\longrightarrow} S\left(\prod_{d \in \mathbb{N}_{\mathbf{d}}} \mathrm{GL}_{t_{d}}(\mathbb{R})_{\Delta}^{d}\right)
$$

As $\prod_{d \in \mathbb{N}_{\mathbf{d}}}\left(\mathrm{O}_{t_{d}}\right)_{\Delta}^{d}$ is a maximal compact subgroup of $\prod_{d \in \mathbb{N}_{\mathbf{d}}} \mathrm{GL}_{t_{d}}(\mathbb{R})_{\Delta}^{d}$, and $S\left(\prod_{d \in \mathbb{N}_{\mathbf{d}}} \mathrm{GL}_{t_{d}}(\mathbb{R})_{\Delta}^{d}\right)$ is normal in $\prod_{d \in \mathbb{N}_{\mathbf{d}}} \mathrm{GL}_{t_{d}}(\mathbb{R})_{\Delta}^{d}$, it follows that $S\left(\prod_{d \in \mathbb{N}_{\mathbf{d}}}\left(\mathrm{O}_{t_{d}}\right)_{\Delta}^{d}\right)$ is a maximal compact subgroup of $S\left(\prod_{d \in \mathbb{N}_{\mathbf{d}}} \mathrm{GL}_{t_{d}}(\mathbb{R})_{\Delta}^{d}\right)$. In view of the above observations it is now clear that for $n \geq 3$,

$$
H^{2}\left(\mathcal{O}_{X}, \mathbb{R}\right) \simeq\left[\mathfrak{z}(\mathfrak{k})^{*}\right]^{K / K^{\circ}} \quad \text { where } \quad K \simeq \prod_{\eta \in \mathbb{E}_{\mathbf{d}}} \mathrm{O}_{t_{\eta}} \times S\left(\prod_{\theta \in \mathbb{O}_{\mathbf{d}}} \mathrm{O}_{t_{\theta}}\right)
$$

Consider the group $A:=S\left(\mathrm{O}_{n_{1}} \times \cdots \times \mathrm{O}_{n_{r}}\right)$ for positive integers $n_{1}, \cdots, n_{r}$. Let $\mathfrak{a}$ be the Lie algebra of $A$. It is then easy to prove (see the proof of Case-2 in [BC1, Theorem 5.6]) that

$$
\operatorname{dim}_{\mathbb{R}}[\mathfrak{z}(\mathfrak{a})]^{A / A^{\circ}}= \begin{cases}1 & \text { if } r=1 \text { and } n_{r}=2 \\ 0 & \text { otherwise. }\end{cases}
$$

It is also immediate that if $B_{1}, B_{2}$ are Lie groups, $B_{3}:=B_{1} \times B_{2}$, and $\mathfrak{b}_{i}, 1 \leq i \leq 3$, is the Lie algebra of $B_{i}$, then

$$
\left[\mathfrak{z}\left(\mathfrak{b}_{3}\right)\right]^{B_{3} / B_{3}^{\circ}} \simeq\left[\mathfrak{z}\left(\mathfrak{b}_{1}\right)\right]^{B_{1} / B_{1}^{\circ}} \oplus\left[\mathfrak{z}\left(\mathfrak{b}_{2}\right)\right]^{B_{2} / B_{2}^{\circ}} .
$$

Now the theorem follows from (4.11), (4.12) and (4.10).

4.3. Second cohomology groups of nilpotent orbits in $\mathfrak{s l}_{n}(\mathbb{H})$. In this subsection we compute the second cohomology groups of nilpotent orbits in $\mathfrak{s l}_{n}(\mathbb{H})$ under the adjoint action of $\mathrm{SL}_{n}(\mathbb{H})$. We will follow notation as defined in $\S 2$. First recall a standard parametrization of $\mathcal{N}\left(\mathrm{SL}_{n}(\mathbb{H})\right)$, the set of all nilpotent orbits in $\mathfrak{s l}_{n}(\mathbb{H})$. Let $X \in \mathfrak{s l}_{n}(\mathbb{H})$ be a nilpotent element with $\mathcal{O}_{X}$ being its orbit in $\mathfrak{s l}_{n}(\mathbb{H})$ under the adjoint action of $\mathrm{SL}_{n}(\mathbb{H})$. First assume that $X \neq 0$. Let $\{X, H, Y\} \subset \mathfrak{s l}_{n}(\mathbb{H})$ be a $\mathfrak{s l}_{2}(\mathbb{R})$-triple. Let $V:=\mathbb{H}^{n}$ be the right $\mathbb{H}$-vector space of column vectors. Let $\left\{d_{1}, \ldots, d_{s}\right\}$ with $d_{1}<\cdots<d_{s}$ be the integers that occur as $\mathbb{R}$-dimensions of 
non-zero irreducible $\operatorname{Span}_{\mathbb{R}}\{X, H, Y\}$-submodules of $V$. Recall that $M(d-1)$ is defined to be the isotypical component of $V$ containing all irreducible $\operatorname{Span}_{\mathbb{R}}\{X, H, Y\}$-submodules of $V$ with highest weight $(d-1)$, and as in $(2.2)$, we set $L(d-1):=V_{Y, 0} \cap M(d-1)$. Recall that the space $L\left(d_{r}-1\right)$ is a $\mathbb{H}$-subspace for $r=1, \ldots, s$. Let $t_{d_{r}}:=\operatorname{dim}_{\mathbb{H}} L\left(d_{r}-1\right)$ for $1 \leq r \leq s$. So $\left[d_{1}^{t_{d_{1}}}, \cdots, d_{s}^{t_{d_{s}}}\right] \in \mathcal{P}(n)$ as $\sum_{r=1}^{s} t_{d_{r}} d_{r}=n$. This gives a map $\Psi_{\mathrm{SL}_{n}(\mathbb{H})}: \mathcal{N}\left(\mathrm{SL}_{n}(\mathbb{H})\right) \longrightarrow \mathcal{P}(n)$. It follows easily that $\Psi_{\mathrm{SL}_{n}(\mathbb{H})}\left(\mathcal{O}_{X}\right) \neq\left[1^{n}\right]$. By declaring $\Psi_{\mathrm{SL}_{n}(\mathbb{H})}\left(\mathcal{O}_{0}\right):=\left[1^{n}\right]$ we now have a surjective map

$$
\Psi_{\mathrm{SL}_{n}(\mathbb{H})}: \mathcal{N}\left(\mathrm{SL}_{n}(\mathbb{H})\right) \longrightarrow \mathcal{P}(n)
$$

Theorem 4.8 ([CoMc, Theorem 9.3.3]). The map $\Psi_{\mathrm{SL}_{n}(\mathbb{H})}$ in (4.13) is a bijection.

As the Lie algebra $\mathfrak{s l}_{1}(\mathbb{H})$ is isomorphic to $\mathfrak{s u}(2)$ which is a compact Lie algebra, we will further assume that $n \geq 2$.

Theorem 4.9. For every nilpotent element $X \in \mathfrak{s l}_{n}(\mathbb{H})$ when $n \geq 2$,

$$
\operatorname{dim}_{\mathbb{R}} H^{2}\left(\mathcal{O}_{X}, \mathbb{R}\right)=0 .
$$

Proof. We assume that $X \neq 0$ because the theorem is obvious when $X=0$.

Suppose that $\Psi_{\mathrm{SL}_{n}(\mathbb{H})}\left(\mathcal{O}_{X}\right)=$ d. Using the notation in Lemma 4.4 and the paragraph preceding it, let $\{X, H, Y\} \subset \mathfrak{s l}_{n}(\mathbb{H})$ be a $\mathfrak{s l}_{2}(\mathbb{R})$-triple. Let $K$ be a maximal compact subgroup in $\mathcal{Z}_{\mathrm{SL}_{n}(\mathbb{H})}(X, H, Y)$. As $\mathrm{Sp}(n)$ is a maximal compact subgroup of $\mathrm{SL}_{n}(\mathbb{H})$, it follows from Theorem 4.2 that

$$
H^{2}\left(\mathcal{O}_{X}, \mathbb{R}\right) \simeq\left[\mathfrak{z}(\mathfrak{k})^{*}\right]^{K / K^{\circ}}
$$

for all $X \neq 0$. Treating $\mathbb{H}^{n}$ as a $\operatorname{Span}_{\mathbb{R}}\{X, H, Y\}$-module via the standard action of $\mathfrak{s l}_{n}(\mathbb{H})$, we construct a $\mathbb{H}$-basis $\mathcal{B}$ as in (4.4), and consider the $\mathbb{R}$-algebra isomorphism $\Lambda_{\mathcal{B}}$ in (4.5). It now follows from Lemma 4.4(2) that the restriction of $\Lambda_{\mathcal{B}}$ induces an isomorphism of Lie groups

$$
\Lambda_{\mathcal{B}}: \mathcal{Z}_{\mathrm{SL}_{n}(\mathbb{H})}(X, H, Y) \stackrel{\sim}{\longrightarrow} S\left(\prod_{d \in \mathbb{N}_{\mathbf{d}}} \mathrm{GL}_{t_{d}}(\mathbb{H})_{\Delta}^{d}\right)
$$

As $\prod_{d \in \mathbb{N}_{\mathbf{d}}} \operatorname{Sp}\left(t_{d}\right)_{\Delta}^{d}$ is a maximal compact subgroup of $\prod_{d \in \mathbb{N}_{\mathbf{d}}} \mathrm{GL}_{t_{d}}(\mathbb{H})_{\Delta}^{d}$, and

$$
\prod_{d \in \mathbb{N}_{\mathbf{d}}} \mathrm{Sp}\left(t_{d}\right)_{\Delta}^{d} \subset S\left(\prod_{d \in \mathbb{N}_{\mathbf{d}}} \mathrm{GL}_{t_{d}}(\mathbb{H})_{\Delta}^{d}\right)
$$

it follows that $\prod_{d \in \mathbb{N}_{\mathbf{d}}} \operatorname{Sp}\left(t_{d}\right)_{\Delta}^{d}$ is a maximal compact subgroup of $S\left(\prod_{d \in \mathbb{N}_{\mathbf{d}}} \mathrm{GL}_{t_{d}}(\mathbb{H})_{\Delta}^{d}\right)$. In particular we have,

$$
K \simeq \prod_{d \in \mathbb{N}_{\mathbf{d}}} \mathrm{Sp}\left(t_{d}\right)
$$

As $\mathfrak{z}(\mathfrak{k})=0$, it now follows from $(4.14)$ that $\operatorname{dim}_{\mathbb{R}} H^{2}\left(\mathcal{O}_{X}, \mathbb{R}\right)=0$.

4.4. Second cohomology groups of nilpotent orbits in $\mathfrak{s u}(p, q)$. Let $n$ be a positive integer and $(p, q)$ be a pair of non-negative integers such that $p+q=n$. The aim in this subsection is to compute the second cohomology groups of nilpotent orbits in $\mathfrak{s u}(p, q)$ under the adjoint action of $\mathrm{SU}(p, q)$. As explained before, we need to address only non-compact groups; so it will be assumed that $p>0$ and $q>0$. Throughout this subsection $\langle\cdot, \cdot\rangle$ denotes the Hermitian form on $\mathbb{C}^{n}$ defined by $\langle x, y\rangle:=\bar{x}^{t} \mathrm{I}_{p, q} y$, where $\mathrm{I}_{p, q}$ is as in (2.1). We will follow notation as defined in $\S 2$.

First recall a standard parametrization of $\mathcal{N}\left(\mathrm{SL}_{n}(\mathbb{C})\right)$, the set of all nilpotent orbits in $\mathfrak{s l}_{n}(\mathbb{C})$. Using Proposition 5.10, this can be done as in Section 4.2 for $\mathcal{N}\left(\mathrm{SL}_{n}(\mathbb{R})\right)$. Let $X^{\prime} \in \mathcal{N}_{\mathfrak{s l}_{n}(\mathbb{C})}$ be a nilpotent element. First assume that $X^{\prime} \neq 0$, and let $\left\{X^{\prime}, H^{\prime}, Y^{\prime}\right\} \subset \mathfrak{s l}_{n}(\mathbb{C})$ be a $\mathfrak{s l}_{2}(\mathbb{R})$ triple. Let $V:=\mathbb{C}^{n}$ be the right $\mathbb{C}$-vector space of column vectors. Let $\left\{c_{1}, \cdots, c_{l}\right\}$, with 
$c_{1}<\cdots<c_{l}$, be the finitely many ordered integers that occur as $\mathbb{R}$-dimension of non-zero irreducible $\operatorname{Span}_{\mathbb{R}}\left\{X^{\prime}, H^{\prime}, Y^{\prime}\right\}$-submodules of $V$. Recall that $M(c-1)$ is defined to be the isotypical component of $V$ containing all irreducible $\operatorname{Span}_{\mathbb{R}}\left\{X^{\prime}, H^{\prime}, Y^{\prime}\right\}$-submodules of $V$ with highest weight $(c-1)$, and as in $(2.2)$, we set $L(c-1):=V_{Y^{\prime}, 0} \cap M(c-1)$. Recall that the space $L\left(c_{r}-1\right)$ is a $\mathbb{C}$-subspace for $1 \leq r \leq l$. Let $t_{c_{r}}:=\operatorname{dim}_{\mathbb{C}} L\left(c_{r}-1\right)$ for $1 \leq r \leq l$. Then as $\sum_{r=1}^{l} t_{c_{r}} c_{r}=n$, we have $\left[c_{1}^{t_{c_{1}}}, \cdots, c_{l}^{t_{c_{l}}}\right] \in \mathcal{P}(n)$. Define $\Psi_{\mathrm{SL}_{n}(\mathbb{C})}\left(\mathcal{O}_{X^{\prime}}\right):=\left[c_{1}^{t_{c_{1}}}, \cdots, c_{l}^{t_{c_{l}}}\right]$. It is easy to see that $\Psi_{\mathrm{SL}_{n}(\mathbb{C})}\left(\mathcal{O}_{X}\right) \neq\left[1^{n}\right]$ as $X \neq 0$. By declaring $\Psi_{\mathrm{SL}_{n}(\mathbb{C})}\left(\mathcal{O}_{0}\right)=\left[1^{n}\right]$ we obtain a bijection $\Psi_{\mathrm{SL}_{n}(\mathbb{C})}: \mathcal{N}\left(\mathrm{SL}_{n}(\mathbb{C})\right) \longrightarrow \mathcal{P}(n)$. As $\mathrm{SU}(p, q) \subset \mathrm{SL}_{n}(\mathbb{C})$, consequently $\mathcal{N}_{\mathfrak{s u}(p, q)} \subset \mathcal{N}_{\mathfrak{s l}_{n}(\mathbb{C})}$, we have the inclusion map $\Theta_{\mathrm{SU}(p, q)}: \mathcal{N}(\mathrm{SU}(p, q)) \longrightarrow \mathcal{N}\left(\mathrm{SL}_{n}(\mathbb{C})\right)$. Let

$$
\Psi_{\mathrm{SU}(p, q)}^{\prime}:=\Psi_{\mathrm{SL}_{n}(\mathbb{C})} \circ \Theta_{\mathrm{SU}(p, q)}: \mathcal{N}(\mathrm{SU}(p, q)) \longrightarrow \mathcal{P}(n)
$$

be the composition.

Let now $X \in \mathfrak{s u}(p, q)$ be a nilpotent element and $\mathcal{O}_{X}$ the nilpotent orbit of $X$ in $\mathfrak{s u}(p, q)$. Assume that $X \neq 0$, and let $\{X, H, Y\} \subset \mathfrak{s u}(p, q)$ be a $\mathfrak{s l}_{2}(\mathbb{R})$-triple. We now apply Proposition 5.14, Remark 5.16(2), and follow the notation used therein. Let $\left\{d_{1}, \cdots, d_{s}\right\}$, with $d_{1}<$ $\cdots<d_{s}$, be the finite ordered set of integers that arise as $\mathbb{R}$-dimension of non-zero irreducible $\operatorname{Span}_{\mathbb{R}}\{X, H, Y\}$-submodules of $V$. Let $t_{d_{r}}:=\operatorname{dim}_{\mathbb{C}} L\left(d_{r}-1\right)$ for $1 \leq r \leq s$. Then we have $\mathbf{d}:=\left[d_{1}^{t_{d_{1}}}, \cdots, d_{s}^{t_{d_{s}}}\right] \in \mathcal{P}(n)$, and moreover, $\Psi_{\mathrm{SU}(p, q)}^{\prime}\left(\mathcal{O}_{X}\right)=\mathbf{d}$.

We next assign $\operatorname{sgn}_{\mathcal{O}_{X}} \in \mathcal{S}_{\mathbf{d}}(p, q)$ to each $\mathcal{O}_{X} \in \mathcal{N}(\mathrm{SU}(p, q))$; see (2.6) for the definition of $\mathcal{S}_{\mathbf{d}}(p, q)$. For each $d \in \mathbb{N}_{\mathbf{d}}$ (see $(2.3)$ for the definition of $\mathbb{N}_{\mathbf{d}}$ ) we will define a $t_{d} \times d$ matrix $\left(m_{i j}^{d}\right)$ in $\mathbf{A}_{d}$ which depends only on the orbit $\mathcal{O}_{X}$ containing $X$; see (2.5) for the definition of $\mathbf{A}_{d}$. For this, recall that the form $(\cdot, \cdot)_{d}: L(d-1) \times L(d-1) \longrightarrow \mathbb{C}$ defined as in (4.6) is Hermitian (respectively, skew-Hermitian) when $d$ is odd (respectively, even). Denoting the signature of $(\cdot, \cdot)_{d}$ by $\left(p_{d}, q_{d}\right)$ we now define

$$
m_{i 1}^{d}:=\left\{\begin{array}{ll}
+1 & \text { if } 1 \leq i \leq p_{d} \\
-1 & \text { if } p_{d}<i \leq t_{d}
\end{array} ; d \in \mathbb{N}_{\mathbf{d}}\right.
$$

and

$$
\begin{aligned}
& m_{i j}^{d}:=(-1)^{j+1} m_{i 1}^{d} \quad \text { if } 1<j \leq d, d \in \mathbb{E}_{\mathbf{d}} \cup \mathbb{O}_{\mathbf{d}}^{1} ; \\
& m_{i j}^{\theta}:=\left\{\begin{array}{ll}
(-1)^{j+1} m_{i 1}^{\theta} & \text { if } 1<j \leq \theta-1 \\
-m_{i 1}^{\theta} & \text { if } j=\theta
\end{array}, \theta \in \mathbb{O}_{\mathbf{d}}^{3} .\right.
\end{aligned}
$$

The above matrices $\left(m_{i j}^{d}\right)$ clearly satisfy the conditions in (Yd.2). We set $\mathbf{s g n}_{\mathcal{O}_{X}}:=\left(\left(m_{i j}^{d_{1}}\right), \cdots\right.$, $\left.\left(m_{i j}^{d_{s}}\right)\right)$. It now follows from the last paragraph of Remark 5.21 that $\mathbf{s g n}_{\mathcal{O}_{X}} \in \mathcal{S}_{\mathbf{d}}(p, q)$. Thus we have the map

$$
\Psi_{\mathrm{SU}(p, q)}: \mathcal{N}(\mathrm{SU}(p, q)) \longrightarrow \mathcal{Y}(p, q), \quad \mathcal{O}_{X} \longmapsto\left(\Psi_{\mathrm{SU}(p, q)}^{\prime}\left(\mathcal{O}_{X}\right), \operatorname{sgn}_{\mathcal{O}_{X}}\right) ;
$$

where $\mathcal{Y}(p, q)$ is as in (2.7). The following theorem is standard.

Theorem 4.10. The above map $\Psi_{\mathrm{SU}(p, q)}: \mathcal{N}(\mathrm{SU}(p, q)) \longrightarrow \mathcal{Y}(p, q)$ is a bijection.

Remark 4.11. Taking into account the error in [CoMc, Lemma 9.3.1] mentioned in Remark 5.21, the parametrization in Theorem 4.10 is a modification of the one in [CoMc, Theorem 9.3.3].

Let $0 \neq X \in \mathcal{N}_{\mathfrak{s u}(p, q)}$, and $\{X, H, Y\}$ be a $\mathfrak{s l}_{2}(\mathbb{R})$-triple in $\mathfrak{s u}(p, q)$. Let $\Psi_{\mathrm{SU}(p, q)}\left(\mathcal{O}_{X}\right)=$ $\left(\mathbf{d}, \operatorname{sgn}_{\mathcal{O}_{X}}\right)$. Then $\Psi_{\mathrm{SU}(p, q)}^{\prime}\left(\mathcal{O}_{X}\right)=\mathbf{d}$. Recall that $\operatorname{sgn}_{\mathcal{O}_{X}}$ determines the signature of $(\cdot, \cdot)_{d}$ on $L(d-1)$ for every $d \in \mathbb{N}_{\mathbf{d}}$; let $\left(p_{d}, q_{d}\right)$ be the signature of $(\cdot, \cdot)_{d}$, for $d \in \mathbb{N}_{\mathbf{d}}$. Let $\left(v_{1}^{d}, \cdots, v_{t_{d}}^{d}\right)$ be an ordered $\mathbb{C}$-basis of $L(d-1)$ as in Proposition 5.14. It now follows from Proposition 5.14(3)(a) that $\left(v_{1}^{d}, \cdots, v_{t_{d}}^{d}\right)$ is an orthogonal basis for $(\cdot, \cdot)_{d}$. We also assume that the vectors in the 
ordered basis $\left(v_{1}^{d}, \cdots, v_{t_{d}}^{d}\right)$ satisfies the properties in Remark 5.16(2). In view of the signature of $(\cdot, \cdot)_{d}$ we may further assume that

$$
\begin{aligned}
\sqrt{-1}\left(v_{j}^{\eta}, v_{j}^{\eta}\right)_{\eta} & =\left\{\begin{array}{ll}
+1 & \text { if } 1 \leq j \leq p_{\eta} \\
-1 & \text { if } p_{\eta}<j \leq t_{\eta}
\end{array} ; \text { when } \eta \in \mathbb{E}_{\mathbf{d}},\right. \\
\left(v_{j}^{\theta}, v_{j}^{\theta}\right)_{\theta} & =\left\{\begin{array}{ll}
+1 & \text { if } 1 \leq j \leq p_{\theta} \\
-1 & \text { if } p_{\theta}<j \leq t_{\theta}
\end{array} ; \text { when } \theta \in \mathbb{O}_{\mathbf{d}} .\right.
\end{aligned}
$$

Let $\left\{\widetilde{w}_{j l}^{d} \mid 1 \leq j \leq t_{d}, 0 \leq l \leq d-1\right\}$ be the $\mathbb{C}$-basis of $M(d-1)$ constructed using $\left(v_{1}^{d}, \cdots, v_{t_{d}}^{d}\right)$ as done in Lemma 5.18. For each $d \in \mathbb{N}_{\mathbf{d}}, 0 \leq l \leq d-1$, set

$$
V^{l}(d):=\operatorname{Span}_{\mathbb{C}}\left\{\widetilde{w}_{1 l}^{d}, \cdots, \widetilde{w}_{t_{d} l}^{d}\right\} .
$$

The ordered basis $\left(\widetilde{w}_{1 l}^{d}, \cdots, \widetilde{w}_{t_{d} l}^{d}\right)$ of $V^{l}(d)$ will be denoted by $\mathcal{C}^{l}(d)$.

Lemma 4.12. The following holds:

$$
\mathcal{Z}_{\mathrm{SU}(p, q)}(X, H, Y)=\left\{g \in \mathrm{SU}(p, q) \mid \begin{array}{c}
g\left(V^{l}(d)\right) \subset V^{l}(d) \text { and } \\
{\left[\left.g\right|_{V^{l}(d)}\right]_{\mathcal{C}^{l}(d)}=\left[\left.g\right|_{V^{0}(d)}\right]_{\mathcal{C}^{0}(d)} \text { for all } d \in \mathbb{N}_{\mathbf{d}}, 0 \leq l<d}
\end{array}\right\} .
$$

Proof. As $\mathcal{Z}_{\mathrm{SU}(p, q)}(X, H, Y)=\mathrm{SU}(p, q) \cap \mathcal{Z}_{\mathrm{SL}_{n}(\mathbb{C})}(X, H, Y)$, using Lemma 4.4(1) it follows that

$$
\mathcal{Z}_{\mathrm{SU}(p, q)}(X, H, Y)=\left\{g \in \mathrm{SU}(p, q) \mid \begin{array}{c}
g\left(X^{l} L(d-1)\right) \subset X^{l} L(d-1) \text { and } \\
{\left[\left.g\right|_{X^{l} L(d-1)}\right]_{\mathcal{B}^{l}(d)}=\left[\left.g\right|_{L(d-1)}\right]_{\mathcal{B}^{0}(d)} \text { for all } d \in \mathbb{N}_{\mathbf{d}}, 0 \leq l<d}
\end{array}\right\} .
$$

For fixed $d \in \mathbb{N}_{\mathbf{d}}$ we consider the $t_{d} \times 1$-column matrices $\left[\widetilde{w}_{j l}^{d}\right]_{1 \leq j \leq t_{d}},\left[\widetilde{w}_{j(d-1-l)}^{d}\right]_{1 \leq j \leq t_{d}}$ and $\left[X^{l} v_{j}^{d}\right]_{1 \leq j \leq t_{d}},\left[X^{d-1-l} v_{j}^{d}\right]_{1 \leq j \leq t_{d}}$. Rewriting the definitions in Lemma 5.18 when $\eta \in \mathbb{E}_{\mathbf{d}}$,

$$
\left[\widetilde{w}_{j l}^{\eta}\right]=\left(\left[X^{l} v_{j}^{\eta}\right]+\left[X^{\eta-1-l} v_{j}^{\eta}\right] \sqrt{-1}\right) \frac{1}{\sqrt{2}} ;\left[\widetilde{w}_{j(\eta-1-l)}^{\eta}\right]=\left(\left[X^{l} v_{j}^{\eta}\right]-\left[X^{\eta-1-l} v_{j}^{\eta}\right] \sqrt{-1}\right) \frac{1}{\sqrt{2}}
$$

for $0 \leq l<\eta / 2$.

Furthermore, when $1 \leq \theta \in \mathbb{O}_{\mathbf{d}}$,

$$
\left[\widetilde{w}_{j l}^{\theta}\right]=\left(\left[X^{l} v_{j}^{\theta}\right]+\left[X^{\theta-1-l} v_{j}^{\theta}\right]\right) \frac{1}{\sqrt{2}} ;\left[\widetilde{w}_{j(\theta-1-l)}^{\theta}\right]=\left(\left[X^{l} v_{j}^{\theta}\right]-\left[X^{\theta-1-l} v_{j}^{\theta}\right]\right) \frac{1}{\sqrt{2}},
$$

for all $0 \leq l<(\theta-1) / 2$, while for $l=(\theta-1) / 2$,

$$
\left[\widetilde{w}_{j(\theta-1) / 2}^{\theta}\right]=\left[X^{(\theta-1) / 2} v_{j}^{\theta}\right] .
$$

When $\theta=1$, then $\left[\widetilde{w}_{j}^{\theta}\right]=\left[v_{j}^{\theta}\right]$.

In particular, if $d \in \mathbb{N}_{\mathbf{d}}$ is fixed, then for every $0 \leq l \leq d-1$ the following holds:

$$
g\left(X^{l} L(d-1)\right) \subset X^{l} L(d-1) \text { if and only if } g\left(V^{l}(d)\right) \subset V^{l}(d),
$$

and moreover,

$$
\left[\left.g\right|_{X^{l} L(d-1)}\right]_{\mathcal{B}^{l}(d)}=\left[\left.g\right|_{L(d-1)}\right]_{\mathcal{B}^{0}(d)} \text { if and only if } \quad\left[\left.g\right|_{V^{l}(d)}\right]_{\mathcal{C}^{l}(d)}=\left[\left.g\right|_{V^{0}(d)}\right]_{\mathcal{C}^{0}(d)} .
$$

In fact, for any $g$ as above, $\left[\left.g\right|_{L(d-1)}\right]_{\mathcal{B}^{0}(d)}=\left[\left.g\right|_{V^{0}(d)}\right]_{\mathcal{C}^{0}(d)}$.

For every $d \in \mathbb{N}_{\mathbf{d}}$ and $0 \leq l \leq d-1$, orderings on the sets $\left\{v \in \mathcal{C}^{l}(d) \mid\langle v, v\rangle>0\right\}$, $\left\{v \in \mathcal{C}^{l}(d) \mid\langle v, v\rangle<0\right\}$, will be constructed. These ordered sets will be denoted by $\mathcal{C}_{+}^{l}(d)$ and $\mathcal{C}_{-}^{l}(d)$ respectively. The construction will be done in three steps according as $d \in \mathbb{E}_{\mathbf{d}}$ or $d \in \mathbb{O}_{\mathbf{d}}^{1}$ or $d \in \mathbb{O}_{\mathbf{d}}^{3}$; see $(2.3)$ and $(2.4)$ for definitions of $\mathbb{E}_{\mathbf{d}}, \mathbb{O}_{\mathbf{d}}^{1}, \mathbb{O}_{\mathbf{d}}^{3}$. 
For each $\eta \in \mathbb{E}_{\mathbf{d}}$ and $0 \leq l \leq \eta-1$, define

$$
\begin{aligned}
& \mathcal{C}_{+}^{l}(\eta):= \begin{cases}\left(\widetilde{w}_{1 l}^{\eta}, \cdots, \widetilde{w}_{p_{\eta} l}^{\eta}\right) & \text { if } l \text { is even } \\
\left(\widetilde{w}_{\left(p_{\eta+1} l\right.}^{\eta}, \cdots, \widetilde{w}_{t_{\eta} l}^{\eta}\right) & \text { if } l \text { is odd }\end{cases} \\
& \mathcal{C}_{-}^{l}(\eta):= \begin{cases}\left(\widetilde{w}_{\left(p_{\eta+1} l\right.}^{\eta}, \cdots, \widetilde{w}_{t_{\eta} l}^{\eta}\right) & \text { if } l \text { is even } \\
\left(\widetilde{w}_{1 l}^{\eta}, \cdots, \widetilde{w}_{p_{\eta} l}^{\eta}\right) & \text { if } l \text { is odd. }\end{cases}
\end{aligned}
$$

For each $\theta \in \mathbb{O}_{\mathbf{d}}^{1}$, define

$$
\mathcal{C}_{+}^{l}(\theta):= \begin{cases}\left(\widetilde{w}_{1 l}^{\theta}, \cdots, \widetilde{w}_{p_{\theta} l}^{\theta}\right) & \text { if } l \text { is even and } 0 \leq l<(\theta-1) / 2 \\ \left(\widetilde{w}_{\left(p_{\theta}+1\right) l}^{\theta}, \cdots, \widetilde{w}_{t_{\theta} l}^{\theta}\right) & \text { if } l \text { is odd and } 0 \leq l<(\theta-1) / 2 \\ \left(\widetilde{w}_{1 l}^{\theta}, \cdots, \widetilde{w}_{p_{\theta} l}^{\theta}\right) & \text { if } l=(\theta-1) / 2 \\ \left(\widetilde{w}_{1 l}^{\theta}, \cdots, \widetilde{w}_{p_{\theta} l}^{\theta}\right) & \text { if } l \text { is odd and }(\theta+1) / 2 \leq l \leq(\theta-1) \\ \left(\widetilde{w}_{\left(p_{\theta}+1\right) l}^{\theta}, \cdots, \widetilde{w}_{t_{\theta} l}^{\theta}\right) & \text { if } l \text { is even and }(\theta+1) / 2 \leq l \leq(\theta-1)\end{cases}
$$

and

$$
\mathcal{C}_{-}^{l}(\theta):= \begin{cases}\left(\widetilde{w}_{\left(p_{\theta}+1\right) l}^{\theta}, \cdots, \widetilde{w}_{t_{\theta} l}^{\theta}\right) & \text { if } l \text { is even and } 0 \leq l<(\theta-1) / 2 \\ \left(\widetilde{w}_{1 l}^{\theta}, \cdots, \widetilde{w}_{p_{\theta} l}^{\theta}\right) & \text { if } l \text { is odd and } 0 \leq l<(\theta-1) / 2 \\ \left(\widetilde{w}_{\left(p_{\theta}+1\right) l}^{\theta}, \cdots, \widetilde{w}_{t_{\theta} l}^{\theta}\right) & \text { if } l=(\theta-1) / 2 \\ \left(\widetilde{w}_{\left(p_{\theta}+1\right) l}^{\theta}, \cdots, \widetilde{w}_{t_{\theta} l}^{\theta}\right) & \text { if } l \text { is odd and }(\theta+1) / 2 \leq l \leq(\theta-1) \\ \left(\widetilde{w}_{1 l}^{\theta}, \cdots, \widetilde{w}_{p_{\theta} l}^{\theta}\right) & \text { if } l \text { is even and }(\theta+1) / 2 \leq l \leq(\theta-1) .\end{cases}
$$

Similarly, for each $\zeta \in \mathbb{O}_{\mathbf{d}}^{3}$, define

$$
\mathcal{C}_{+}^{l}(\zeta):= \begin{cases}\left(\widetilde{w}_{1 l}^{\zeta}, \cdots, \widetilde{w}_{p_{\zeta} l}^{\zeta}\right) & \text { if } l \text { is even and } 0 \leq l<(\zeta-1) / 2 \\ \left(\widetilde{w}_{\left(p_{\zeta}+1\right) l}^{\zeta}, \cdots, \widetilde{w}_{t_{\zeta l}}^{\zeta}\right) & \text { if } l \text { is odd and } 0 \leq l<(\zeta-1) / 2 \\ \left(\widetilde{w}_{\left(p_{\zeta}+1\right) l}^{\zeta}, \cdots, \widetilde{w}_{t_{\zeta l} l}^{\zeta}\right) & \text { if } l=(\zeta-1) / 2 \\ \left(\widetilde{w}_{\left(p_{\zeta}+1\right) l}^{\zeta}, \cdots, \widetilde{w}_{t_{\zeta l} l}^{\zeta}\right) & \text { if } l \text { is even and }(\zeta+1) / 2 \leq l \leq(\zeta-1) \\ \left(\widetilde{w}_{1 l}^{\zeta}, \cdots, \widetilde{w}_{p_{\zeta} l}^{\zeta}\right) & \text { if } l \text { is odd and }(\zeta+1) / 2 \leq l \leq(\zeta-1)\end{cases}
$$

and

$$
\mathcal{C}_{-}^{l}(\zeta):= \begin{cases}\left(\widetilde{w}_{\left(p_{\zeta}+1\right) l}^{\zeta}, \cdots, \widetilde{w}_{t_{\zeta} l}^{\zeta}\right) & \text { if } l \text { is even and } 0 \leq l<(\zeta-1) / 2 \\ \left(\widetilde{w}_{1 l}^{\zeta}, \cdots, \widetilde{w}_{p_{\zeta} l}^{\zeta}\right) & \text { if } l \text { is odd and } 0 \leq l<(\zeta-1) / 2 \\ \left(\widetilde{w}_{1 l}^{\zeta}, \cdots, \widetilde{w}_{p_{\zeta} l}^{\zeta}\right) & \text { if } l=(\zeta-1) / 2 \\ \left(\widetilde{w}_{1 l}^{\zeta}, \cdots, \widetilde{w}_{p_{\zeta} l}^{\zeta}\right) & \text { if } l \text { is even and }(\zeta+1) / 2 \leq l \leq(\zeta-1) \\ \left(\widetilde{w}_{\left(p_{\zeta}+1\right) l}^{\zeta}, \cdots, \widetilde{w}_{t_{\zeta} l}^{\zeta}\right) & \text { if } l \text { is odd and }(\zeta+1) / 2 \leq l \leq(\zeta-1) .\end{cases}
$$

For all $d \in \mathbb{N}_{\mathbf{d}}$ and $0 \leq l \leq d-1$, define

$$
V_{+}^{l}(d):=\operatorname{Span}_{\mathbb{C}}\left\{v \in \mathcal{C}^{l}(d) \mid\langle v, v\rangle>0\right\}, \quad V_{-}^{l}(d):=\operatorname{Span}_{\mathbb{C}}\left\{v \in \mathcal{C}^{l}(d) \mid\langle v, v\rangle<0\right\} .
$$


It can be verified using (4.17), (4.18) together with the orthogonality relations in Lemma 5.18 that $\mathcal{C}_{+}^{l}(d)$ (respectively, $\mathcal{C}_{-}^{l}(d)$ ) is indeed an ordered set based on the (unordered) set

$$
\left\{v \in \mathcal{C}^{l}(d) \mid\langle v, v\rangle>0\right\}
$$

(respectively, $\left\{v \in \mathcal{C}^{l}(d) \mid\langle v, v\rangle<0\right\}$ ) for all $d \in \mathbb{N}_{\mathbf{d}}$ and $0 \leq l \leq d-1$. In particular, $\mathcal{C}_{+}^{l}(d)$ and $\mathcal{C}_{-}^{l}(d)$ are ordered bases of $V_{+}^{l}(d)$ and $V_{-}^{l}(d)$ respectively, for all $d \in \mathbb{N}_{\mathbf{d}}, 0 \leq l \leq d-1$.

In the next lemma we specify a maximal compact subgroup of $\mathcal{Z}_{\mathrm{SU}(p, q)}(X, H, Y)$ which will be used in Proposition 4.14. For notational convenience, we will use $(-1)^{l}$ to denote the sign ' + ' or the sign '-' depending on whether $l$ is an even integer or an odd integer.

Lemma 4.13. Let $K$ be the subgroup of $\mathcal{Z}_{\mathrm{SU}(p, q)}(X, H, Y)$ consisting of all $g \in \mathcal{Z}_{\mathrm{SU}(p, q)}(X, H, Y)$ satisfying the following conditions:

(1) $g\left(V_{+}^{l}(d)\right) \subset V_{+}^{l}(d)$ and $g\left(V_{-}^{l}(d)\right) \subset V_{-}^{l}(d)$, for all $d \in \mathbb{N}_{\mathbf{d}}$ and $0 \leq l \leq d-1$.

(2) When $\eta \in \mathbb{E}_{\mathbf{d}}$,

$$
\begin{aligned}
{\left[\left.g\right|_{V_{+}^{0}(\eta)}\right]_{\mathcal{C}_{+}^{0}(\eta)} } & =\left[\left.g\right|_{V_{(-1)^{l}}^{l}(\eta)}\right]_{\mathcal{C}_{(-1)^{l}}^{l}(\eta)} \quad ; \text { for all } 0 \leq l \leq \eta-1 . \\
{\left[\left.g\right|_{V_{-}^{0}(\eta)}\right]_{\mathcal{C}_{-}^{0}(\eta)} } & =\left[\left.g\right|_{V_{(-1)^{l}+1}^{l}(\eta)}\right]_{\mathcal{C}_{(-1)^{l} l+1}^{l}(\eta)}
\end{aligned}
$$

(3) When $\theta \in \mathbb{O}_{\mathbf{d}}^{1}$,

$$
\begin{aligned}
& {\left[\left.g\right|_{V_{+}^{0}(\theta)}\right]_{\mathcal{C}_{+}^{0}(\theta)}= \begin{cases}{\left[\left.g\right|_{V_{(-1)^{l}}^{l}(\theta)}\right]_{\mathcal{C}_{(-1)^{l}}^{l}}} & \text { for all } 0 \leq l<(\theta-1) / 2 \\
{\left[\left.g\right|_{V_{+}^{(\theta-1) / 2}(\theta)}\right]_{\mathcal{C}_{+}^{(\theta-1) / 2}(\theta)}} & \\
{\left[\left.g\right|_{V_{(-1)^{l+1}}^{l}(\theta)}\right]_{\mathcal{C}_{(-1)^{l+1}}^{l}(\theta)}} & \text { for all }(\theta-1) / 2<l \leq \theta-1,\end{cases} } \\
& {\left[\left.g\right|_{V_{-}^{0}(\theta)}\right]_{\mathcal{C}_{-}^{0}(\theta)}= \begin{cases}{\left[\left.g\right|_{V_{(-1)^{l+1}}^{l}(\theta)}\right]_{\mathcal{C}_{(-1)^{l}}^{l} l+1}(\theta)} & \text { for all } 0 \leq l<(\theta-1) / 2 \\
{\left[\left.g\right|_{V_{-}^{(\theta-1) / 2}(\theta)}\right]_{\mathcal{C}_{-}^{(\theta-1) / 2}(\theta)}} & \\
{\left[\left.g\right|_{V_{(-1)^{l}}^{l}(\theta)}\right]_{\mathcal{C}_{(-1)^{l}}^{l}(\theta)}} & \text { for all }(\theta-1) / 2<l \leq \theta-1 .\end{cases} }
\end{aligned}
$$

(4) When $\zeta \in \mathbb{O}_{\mathbf{d}}^{3}$,

$$
\begin{aligned}
& {\left[\left.g\right|_{V_{+}^{0}(\zeta)}\right]_{\mathcal{C}_{+}^{0}(\zeta)}=} \begin{cases}{\left[\left.g\right|_{V_{(-1)^{l}}^{l}(\zeta)}\right]_{\mathcal{C}_{(-1)^{l}}^{l}}} & \text { for all } 0 \leq l<(\zeta-1) / 2 \\
{\left[\left.g\right|_{V_{-}^{(\zeta-1) / 2}(\zeta)}\right]_{\mathcal{C}_{-}^{(\zeta-1) / 2}(\zeta)}} & \text { for all }(\zeta-1) / 2<l \leq \zeta-1, \\
{\left[\left.g\right|_{V_{(-1)^{l+1}}^{l}(\zeta)}\right]_{\mathcal{C}_{(-1)^{l+1}}^{l}(\zeta)}} & \text { for all } 0 \leq l<(\zeta-1) / 2\end{cases} \\
& {\left[\left.g\right|_{V_{-}^{0}(\zeta)}\right]_{\mathcal{C}_{-}^{0}(\zeta)}= \begin{cases}{\left[\left.g\right|_{V_{(-1)^{l+1}}^{l}(\zeta)}\right]_{\mathcal{C}_{(-1)^{l+1}}^{l}(\zeta)}} \\
{\left[\left.g\right|_{V_{+}^{(\zeta-1) / 2}(\zeta)}\right]_{\mathcal{C}_{+}^{(\zeta-1) / 2}(\zeta)}} \\
{\left[\left.g\right|_{V_{(-1)^{l}}^{l}(\zeta)}\right]_{\mathcal{C}_{(-1)^{l}}^{l}(\zeta)}} & \text { for all }(\zeta-1) / 2<l \leq \zeta-1 .\end{cases} }
\end{aligned}
$$


Then $K$ is a maximal compact subgroup of $\mathcal{Z}_{\mathrm{SU}(p, q)}(X, H, Y)$.

Proof. In view of the description of $\mathcal{Z}_{\mathrm{SU}(p, q)}(X, H, Y)$ in the Lemma 4.12 we see that its subgroup

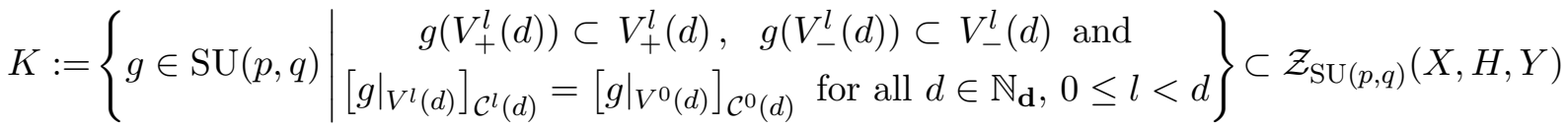
is maximal compact. Thus it suffices show that if $g \in \mathrm{SU}(p, q)$ and $g\left(V_{+}^{l}(d)\right) \subset V_{+}^{l}(d), g\left(V_{-}^{l}(d)\right)$ $\subset V_{-}^{l}(d)$, then $\left[\left.g\right|_{V^{l}(d)}\right]_{\mathcal{C}^{l}(d)}=\left[\left.g\right|_{V^{0}(d)}\right]_{\mathcal{C}^{0}(d)}$ for all $0 \leq l \leq d-1, d \in \mathbb{N}_{\mathbf{d}}$ if and only if $g$ satisfies the conditions (2), (3) and (4) in the statement of the lemma. To do this, we first record the following relations among the ordered sets $\mathcal{C}^{l}(d), \mathcal{C}_{(-1)^{l+1}}^{l}(d)$ and $\mathcal{C}_{(-1)^{l}}^{l}(d)$ for all $d \in \mathbb{N}_{\mathbf{d}}$ : When $\eta \in \mathbb{E}_{\mathbf{d}}$

$$
\mathcal{C}^{l}(\eta)=\mathcal{C}_{(-1)^{l}}^{l}(\eta) \vee \mathcal{C}_{(-1)^{l+1}}^{l}(\eta) \text { for } 0 \leq l \leq \eta-1
$$

When $\theta \in \mathbb{O}_{\mathbf{d}}^{1}$,

$$
\mathcal{C}^{l}(\theta)= \begin{cases}\mathcal{C}_{(-1)^{l}}^{l}(\theta) \vee \mathcal{C}_{(-1)^{l+1}}^{l}(\theta) & \text { for all } 0 \leq l<(\theta-1) / 2 \\ \mathcal{C}_{+1}^{(\theta-1) / 2}(\theta) \vee \mathcal{C}_{-1}^{(\theta-1) / 2}(\theta) & \text { for } l=(\theta-1) / 2 \\ \mathcal{C}_{(-1)^{l+1}}^{l}(\theta) \vee \mathcal{C}_{(-1)^{l}}^{l}(\theta) & \text { for all }(\theta-1) / 2<l \leq \theta-1\end{cases}
$$

When $\zeta \in \mathbb{O}_{\mathbf{d}}^{3}$,

$$
\mathcal{C}^{l}(\zeta)= \begin{cases}\mathcal{C}_{(-1)^{l}}^{l}(\zeta) \vee \mathcal{C}_{(-1)^{l+1}}^{l}(\zeta) & \text { for all } 0 \leq l<(\zeta-1) / 2 \\ \mathcal{C}_{-1}^{(\zeta-1) / 2}(\zeta) \vee \mathcal{C}_{+1}^{(\zeta-1) / 2}(\zeta) & \text { for } l=(\zeta-1) / 2 \\ \mathcal{C}_{(-1)^{l+1}}^{l}(\zeta) \vee \mathcal{C}_{(-1)^{l}}^{l}(\zeta) & \text { for all }(\zeta-1) / 2<l \leq \zeta-1\end{cases}
$$

Assuming that $g \in \mathrm{SU}(p, q), g\left(V_{+}^{l}(d)\right) \subset V_{+}^{l}(d), g\left(V_{-}^{l}(d)\right) \subset V_{-}^{l}(d)$ and

$$
\left[\left.g\right|_{V^{l}(d)}\right]_{\mathcal{C}^{l}(d)}=\left[\left.g\right|_{V^{0}(d)}\right]_{\mathcal{C}^{0}(d)}
$$

for all $0 \leq l \leq d-1, d \in \mathbb{N}_{\mathbf{d}}$, we next show that $g$ satisfies the conditions (2), (3) and (4) in the lemma.

In view of (4.23), for all $\eta \in \mathbb{E}_{\mathbf{d}}$,

$$
\begin{gathered}
{\left[\left.g\right|_{V^{l}(\eta)}\right]_{\mathcal{C}_{(-1)^{l}}^{l}(\eta) \vee \mathcal{C}_{(-1)^{l+1}}^{l}(\eta)}=\left[\left.g\right|_{V^{l}(\eta)}\right]_{\mathcal{C}^{l}(\eta)}=\left[\left.g\right|_{V^{0}(\eta)}\right]_{\mathcal{C}^{0}(\eta)}} \\
=\left(\begin{array}{cc}
{\left[\left.g\right|_{V_{+1}^{0}(\eta)}\right]_{\mathcal{C}_{+1}^{0}(\eta)}} & 0 \\
0 & {\left[\left.g\right|_{V_{-1}^{0}(\eta)}\right]_{\mathcal{C}_{-1}^{0}(\eta)}}
\end{array}\right)
\end{gathered}
$$

Thus for all $\eta \in \mathbb{E}_{\mathbf{d}}$ and $0 \leq l \leq \eta-1$,

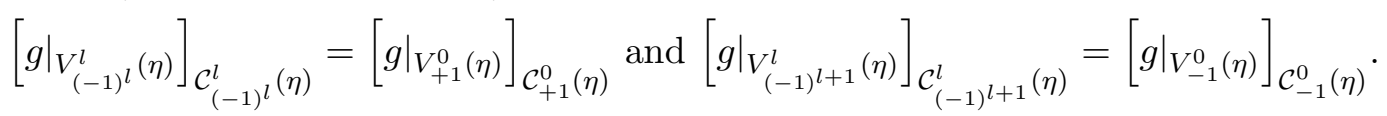

Hence, (2) of the lemma holds.

In view of $(4.24)$, for all $\theta \in \mathbb{O}_{\mathbf{d}}^{1}$ and $0 \leq l<(\theta-1) / 2$,

$$
\begin{gathered}
{\left[\left.g\right|_{V^{l}(\theta)}\right]_{\mathcal{C}_{(-1)^{l}}^{l}(\theta) \vee \mathcal{C}_{(-1)^{l+1}}^{l}(\theta)}=\left[\left.g\right|_{V^{l}(\theta)}\right]_{\mathcal{C}^{l}(\theta)}=\left[\left.g\right|_{V^{0}(\theta)}\right]_{\mathcal{C}^{0}(\theta)}} \\
=\left(\begin{array}{cc}
{\left[\left.g\right|_{V_{+1}^{0}(\theta)}\right]_{\mathcal{C}_{+1}^{0}(\theta)}} & 0 \\
0 & {\left[\left.g\right|_{V_{-1}^{0}(\theta)}\right]_{\mathcal{C}_{-1}^{0}(\theta)}}
\end{array}\right) .
\end{gathered}
$$


Therefore if $\theta \in \mathbb{O}_{\mathbf{d}}^{1}$, then for all $0 \leq l<(\theta-1) / 2$,

$$
\left[\left.g\right|_{V_{(-1)^{l}}^{l}(\theta)}\right]_{\mathcal{C}_{(-1)^{l}}^{l}(\theta)}=\left[\left.g\right|_{V_{+1}^{0}(\theta)}\right]_{\mathcal{C}_{+1}^{0}(\theta)} \text { and }\left[\left.g\right|_{V_{(-1)^{l+1}}^{l}(\theta)}\right]_{\mathcal{C}_{(-1)^{l+1}}^{l}(\theta)}=\left[\left.g\right|_{V_{-1}^{0}(\theta)}\right]_{\mathcal{C}_{-1}^{0}(\theta)} \text {. }
$$

From (4.24), we have

$$
\begin{gathered}
{\left[\left.g\right|_{V^{(\theta-1) / 2}(\theta)}\right]_{\mathcal{C}_{+}^{(\theta-1) / 2}(\theta) \vee \mathcal{C}_{-}^{(\theta-1) / 2}(\theta)}=\left[\left.g\right|_{V^{(\theta-1) / 2}(\theta)}\right]_{\mathcal{C}^{(\theta-1) / 2}(\theta)}=\left[\left.g\right|_{V^{0}(\theta)}\right]_{\mathcal{C}^{0}(\theta)}} \\
=\left(\begin{array}{cc}
{\left[\left.g\right|_{V_{+1}^{0}(\theta)}\right]_{\mathcal{C}_{+1}^{0}(\theta)}} & 0 \\
0 & {\left[\left.g\right|_{V_{-1}^{0}(\theta)}\right]_{\mathcal{C}_{-1}^{0}(\theta)}}
\end{array}\right) .
\end{gathered}
$$

Thus,

$$
\left[\left.g\right|_{V_{+}^{(\theta-1) / 2}(\theta)}\right]_{\mathcal{C}_{+}^{(\theta-1) / 2}(\theta)}=\left[\left.g\right|_{V_{+}^{0}(\theta)}\right]_{\mathcal{C}_{+}^{0}(\theta)},\left[\left.g\right|_{V_{-}^{(\theta-1) / 2}(\theta)}\right]_{\mathcal{C}_{-}^{(\theta-1) / 2}(\theta)}=\left[\left.g\right|_{V_{-}^{0}(\theta)}\right]_{\mathcal{C}_{-}^{0}(\theta)} .
$$

When $(\theta-1) / 2<l \leq \theta-1$, we have

$$
\begin{aligned}
& {\left[\left.g\right|_{V^{l}(\theta)}\right]_{\mathcal{C}_{(-1)^{l} l+1}^{l}(\theta) \vee \mathcal{C}_{(-1)^{l}}^{l}(\theta)}=\left[\left.g\right|_{V^{l}(\theta)}\right]_{\mathcal{C}^{l}(\theta)}=\left[\left.g\right|_{V^{0}(\theta)}\right]_{\mathcal{C}^{0}(\theta)}} \\
& =\left(\begin{array}{cc}
{\left[\left.g\right|_{V_{+1}^{0}(\theta)}\right]_{\mathcal{C}_{+1}^{0}(\theta)}} & 0 \\
0 & {\left[\left.g\right|_{V_{-1}^{0}(\theta)}\right]_{\mathcal{C}_{-1}^{0}(\theta)}}
\end{array}\right) .
\end{aligned}
$$

Thus if $\theta \in \mathbb{O}_{\mathbf{d}}^{1}$, then for all $(\theta-1) / 2<l \leq \theta-1$,

$$
\left[\left.g\right|_{V_{(-1)^{l+1}}^{l}(\theta)}\right]_{\mathcal{C}_{(-1)^{l} l+1}^{l}(\theta)}=\left[\left.g\right|_{V_{+1}^{0}(\theta)}\right]_{\mathcal{C}_{+1}^{0}(\theta)} \text { and }\left[\left.g\right|_{V_{(-1)^{l}}^{l}(\theta)}\right]_{\mathcal{C}_{(-1)^{l}}^{l}(\theta)}=\left[\left.g\right|_{V_{-1}^{0}(\theta)}\right]_{\mathcal{C}_{-1}^{0}(\theta)} \text {. }
$$

Hence, (3) of the lemma holds.

When $g\left(V_{+}^{l}(\zeta)\right) \subset V_{+}^{l}(\zeta), g\left(V_{-}^{l}(\zeta)\right) \subset V_{-}^{l}(\zeta)$ and $\left[\left.g\right|_{V^{l}(\zeta)}\right]_{\mathcal{C}^{l}(\zeta)}=\left[\left.g\right|_{V^{0}(\zeta)}\right]_{\mathcal{C}^{0}(\zeta)}$ for all $0 \leq l \leq$ $\zeta-1, \zeta \in \mathbb{O}_{\mathbf{d}}^{3}$, using (4.25) it follows, similarly as above, that (4) of the lemma holds.

To prove the opposite implication, we assume that $g$ satisfies the conditions $g\left(V_{+}^{l}(d)\right) \subset V_{+}^{l}(d)$, $g\left(V_{-}^{l}(d)\right) \subset V_{-}^{l}(d)$ as well as the conditions (2), (3), (4) of the lemma. Using the relations (4.23), $(4.24)$ and $(4.25)$ it is now straightforward to check that $\left[\left.g\right|_{V^{l}(d)}\right]_{\mathcal{C}^{l}(d)}=\left[\left.g\right|_{V^{0}(d)}\right]_{\mathcal{C}^{0}(d)}$ for all $0 \leq l \leq d-1, d \in \mathbb{N}_{\mathbf{d}}$. This completes the proof of the lemma.

We now introduce some notation which will be required to state Proposition 4.14. For $d \in \mathbb{N}_{\mathbf{d}}$, define

$$
\mathcal{C}_{+}(d):=\mathcal{C}_{+}^{0}(d) \vee \cdots \vee \mathcal{C}_{+}^{d-1}(d) \text { and } \mathcal{C}_{-}(d):=\mathcal{C}_{-}^{0}(d) \vee \cdots \vee \mathcal{C}_{-}^{d-1}(d) .
$$

Let $\alpha:=\# \mathbb{E}_{\mathbf{d}}, \beta:=\# \mathbb{O}_{\mathbf{d}}^{1}$ and $\gamma:=\# \mathbb{O}_{\mathbf{d}}^{3}$. We enumerate

$$
\mathbb{E}_{\mathbf{d}}=\left\{\eta_{i} \mid 1 \leq i \leq \alpha\right\}
$$

such that $\eta_{i}<\eta_{i+1}$,

$$
\mathbb{O}_{\mathbf{d}}^{1}=\left\{\theta_{j} \mid 1 \leq j \leq \beta\right\}
$$

such that $\theta_{j}<\theta_{j+1}$ and similarly

$$
\mathbb{O}_{\mathbf{d}}^{3}=\left\{\zeta_{j} \mid 1 \leq j \leq \gamma\right\}
$$

such that $\zeta_{j}<\zeta_{j+1}$. Now define

$$
\begin{aligned}
\mathcal{E}_{+}:=\mathcal{C}_{+}\left(\eta_{1}\right) \vee \cdots \vee \mathcal{C}_{+}\left(\eta_{\alpha}\right) ; \quad \mathcal{O}_{+}^{1}:=\mathcal{C}_{+}\left(\theta_{1}\right) \vee \cdots \vee \mathcal{C}_{+}\left(\theta_{\beta}\right) ; \quad \mathcal{O}_{+}^{3}:=\mathcal{C}_{+}\left(\zeta_{1}\right) \vee \cdots \vee \mathcal{C}_{+}\left(\zeta_{\gamma}\right) \\
\mathcal{E}_{-}:=\mathcal{C}_{-}\left(\eta_{1}\right) \vee \cdots \vee \mathcal{C}_{-}\left(\eta_{\alpha}\right) ; \mathcal{O}_{-}^{1}:=\mathcal{C}_{-}\left(\theta_{1}\right) \vee \cdots \vee \mathcal{C}_{-}\left(\theta_{\beta}\right) \text { and } \mathcal{O}_{-}^{3}:=\mathcal{C}_{-}\left(\zeta_{1}\right) \vee \cdots \vee \mathcal{C}_{-}\left(\zeta_{\gamma}\right)
\end{aligned}
$$


Finally we define

$$
\mathcal{H}_{+}:=\mathcal{E}_{+} \vee \mathcal{O}_{+}^{1} \vee \mathcal{O}_{+}^{3}, \quad \mathcal{H}_{-}:=\mathcal{E}_{-} \vee \mathcal{O}_{-}^{1} \vee \mathcal{O}_{-}^{3} \text { and } \mathcal{H}:=\mathcal{H}_{+} \vee \mathcal{H}_{-}
$$

It is clear that $\mathcal{H}$ is a standard orthogonal basis with $\mathcal{H}_{+}=\{v \in \mathcal{H} \mid\langle v, v\rangle=1\}$ and $\mathcal{H}_{-}=\{v \in \mathcal{H} \mid\langle v, v\rangle=-1\}$. In particular, $\# \mathcal{H}_{+}=p$ and $\# \mathcal{H}_{-}=q$. From the definition of the $\mathcal{H}_{+}$and $\mathcal{H}_{-}$we have the following relations:

$$
\sum_{i=1}^{\alpha} \frac{\eta_{i}}{2} t_{\eta_{i}}+\sum_{j=1}^{\beta}\left(\frac{\theta_{j}+1}{2} p_{\theta_{j}}+\frac{\theta_{j}-1}{2} q_{\theta_{j}}\right)+\sum_{k=1}^{\gamma}\left(\frac{\zeta_{k}-1}{2} p_{\zeta_{k}}+\frac{\zeta_{k}+1}{2} q_{\zeta_{k}}\right)=p
$$

and

$$
\sum_{i=1}^{\alpha} \frac{\eta_{i}}{2} t_{\eta_{i}}+\sum_{j=1}^{\beta}\left(\frac{\theta_{j}-1}{2} p_{\theta_{j}}+\frac{\theta_{j}+1}{2} q_{\theta_{j}}\right)+\sum_{k=1}^{\gamma}\left(\frac{\zeta_{k}+1}{2} p_{\zeta_{k}}+\frac{\zeta_{k}-1}{2} q_{\zeta_{k}}\right)=q .
$$

The $\mathbb{C}$-algebra

$$
\prod_{i=1}^{\alpha}\left(\mathrm{M}_{p_{\eta_{i}}}(\mathbb{C}) \times \mathrm{M}_{q_{\eta_{i}}}(\mathbb{C})\right) \times \prod_{j=1}^{\beta}\left(\mathrm{M}_{p_{\theta_{j}}}(\mathbb{C}) \times \mathrm{M}_{q_{\theta_{j}}}(\mathbb{C})\right) \times \prod_{k=1}^{\gamma}\left(\mathrm{M}_{p_{\zeta_{k}}}(\mathbb{C}) \times \mathrm{M}_{{\zeta_{\zeta}}_{\zeta_{k}}}(\mathbb{C})\right)
$$

is embedded into $\mathrm{M}_{p}(\mathbb{C})$ and $\mathrm{M}_{q}(\mathbb{C})$ in the following two ways:

$$
\mathbf{D}_{p}: \prod_{i=1}^{\alpha}\left(\mathrm{M}_{p_{\eta_{i}}}(\mathbb{C}) \times \mathrm{M}_{q_{\eta_{i}}}(\mathbb{C})\right) \times \prod_{j=1}^{\beta}\left(\mathrm{M}_{p_{\theta_{j}}}(\mathbb{C}) \times \mathrm{M}_{q_{\theta_{j}}}(\mathbb{C})\right) \times \prod_{k=1}^{\gamma}\left(\mathrm{M}_{p_{\zeta_{k}}}(\mathbb{C}) \times \mathrm{M}_{{\zeta_{\zeta}}_{k}}(\mathbb{C})\right) \longrightarrow \mathrm{M}_{p}(\mathbb{C})
$$

is defined by

$$
\begin{gathered}
\left(A_{\eta_{1}}, B_{\eta_{1}}, \cdots, A_{\eta_{\alpha}}, B_{\eta_{\alpha}} ; C_{\theta_{1}}, D_{\theta_{1}}, \cdots, C_{\theta_{\beta}}, D_{\theta_{\beta}} ; E_{\zeta_{1}}, F_{\zeta_{1}}, \cdots, E_{\zeta_{\gamma}}, F_{\zeta_{\gamma}}\right) \\
\longmapsto \bigoplus_{i=1}^{\alpha}\left(A_{\eta_{i}} \oplus B_{\eta_{i}}\right)^{\eta_{i} / 2} \\
\oplus \bigoplus_{j=1}^{\beta}\left(\left(C_{\theta_{j}} \oplus D_{\theta_{j}}\right)^{\frac{\theta_{j}-1}{4}} \oplus C_{\theta_{j}} \oplus\left(C_{\theta_{j}} \oplus D_{\theta_{j}}\right)^{\frac{\theta_{j}-1}{4}}\right) \oplus \bigoplus_{k=1}^{\gamma}\left(\left(E_{\zeta_{k}} \oplus F_{\zeta_{k}}\right)^{\frac{\zeta_{k}+1}{4}} \oplus\left(F_{\zeta_{k}} \oplus E_{\zeta_{k}}\right)^{\frac{\zeta_{k}-3}{4}} \oplus F_{\zeta_{k}}\right), \\
\text { and } \\
\mathbf{D}_{q}: \prod_{i=1}^{\alpha}\left(\mathrm{M}_{p_{\eta_{i}}}(\mathbb{C}) \times \mathrm{M}_{q_{\eta_{i}}}(\mathbb{C})\right) \times \prod_{j=1}^{\beta}\left(\mathrm{M}_{p_{\theta_{j}}}(\mathbb{C}) \times \mathrm{M}_{q_{\theta_{j}}}(\mathbb{C})\right) \times \prod_{k=1}^{\gamma}\left(\mathrm{M}_{p_{\zeta_{k}}}(\mathbb{C}) \times \mathrm{M}_{q_{\zeta_{k}}}(\mathbb{C})\right) \longrightarrow \mathrm{M}_{q}(\mathbb{C})
\end{gathered}
$$

is defined by

$$
\begin{aligned}
\left(A_{\eta_{1}}, B_{\eta_{1}}, \cdots,\right. & \left.A_{\eta_{\alpha}}, B_{\eta_{\alpha}} ; C_{\theta_{1}}, D_{\theta_{1}}, \cdots, C_{\theta_{\beta}}, D_{\theta_{\beta}} ; E_{\zeta_{1}}, F_{\zeta_{1}}, \cdots, E_{\zeta_{\gamma}}, F_{\zeta_{\gamma}}\right) \\
& \longmapsto \bigoplus_{i=1}^{\alpha}\left(B_{\eta_{i}} \oplus A_{\eta_{i}}\right)^{\eta_{i} / 2} \oplus \bigoplus_{j=1}^{\beta}\left(\left(D_{\theta_{j}} \oplus C_{\theta_{j}}\right)^{\frac{\theta_{j}-1}{4}} \oplus D_{\theta_{j}} \oplus\left(D_{\theta_{j}} \oplus C_{\theta_{j}}\right)^{\frac{\theta_{j}-1}{4}}\right) \\
& \oplus \bigoplus_{k=1}^{\gamma}\left(\left(F_{\zeta_{k}} \oplus E_{\zeta_{k}}\right)^{\frac{\zeta_{k}+1}{4}} \oplus\left(E_{\zeta_{k}} \oplus F_{\zeta_{k}}\right)^{\frac{\zeta_{k}-3}{4}} \oplus E_{\zeta_{k}}\right) .
\end{aligned}
$$

Define the characters

$$
\begin{gathered}
\chi_{p}: \prod_{i=1}^{\alpha}\left(\mathrm{GL}_{p_{\eta_{i}}}(\mathbb{C}) \times \mathrm{GL}_{q_{\eta_{i}}}(\mathbb{C})\right) \times \prod_{j=1}^{\beta}\left(\mathrm{GL}_{p_{\theta_{j}}}(\mathbb{C}) \times \mathrm{GL}_{q_{\theta_{j}}}(\mathbb{C})\right) \times \prod_{k=1}^{\gamma}\left(\mathrm{GL}_{p_{\zeta_{k}}}(\mathbb{C}) \times \mathrm{GL}_{q_{\zeta_{k}}}(\mathbb{C})\right) \longrightarrow \mathbb{C}^{*} \\
\left(A_{\eta_{1}}, B_{\eta_{1}}, \cdots, A_{\eta_{\alpha}}, B_{\eta_{\alpha}} ; C_{\theta_{1}}, D_{\theta_{1}}, \cdots, C_{\theta_{\beta}}, D_{\theta_{\beta}} ; E_{\zeta_{1}}, F_{\zeta_{1}}, \cdots, E_{\zeta_{\gamma}}, F_{\zeta_{\gamma}}\right)
\end{gathered}
$$




$$
\longmapsto \prod_{i=1}^{\alpha}\left(\operatorname{det} A_{\eta_{i}}^{\eta_{i} / 2} \operatorname{det} B_{\eta_{i}}^{\eta_{i} / 2}\right) \prod_{j=1}^{\beta}\left(\operatorname{det} C_{\theta_{j}{ }^{2}}^{\frac{\theta_{j}+1}{2}} \operatorname{det} D_{\theta_{j}{ }^{2}}^{\frac{\theta_{j}-1}{2}}\right) \prod_{k=1}^{\gamma}\left(\operatorname{det} E_{\zeta_{k}}^{\frac{\zeta_{k}-1}{2}} \operatorname{det} F_{\zeta_{k}}^{\frac{\zeta_{k}+1}{2}}\right)
$$

and

$$
\begin{aligned}
\chi_{q}: \prod_{i=1}^{\alpha}\left(\mathrm{GL}_{p_{\eta_{i}}}(\mathbb{C}) \times \mathrm{GL}_{q_{\eta_{i}}}(\mathbb{C})\right) \times \prod_{j=1}^{\beta}\left(\mathrm{GL}_{p_{\theta_{j}}}(\mathbb{C}) \times \mathrm{GL}_{q_{\theta_{j}}}(\mathbb{C})\right) \times \prod_{k=1}^{\gamma}\left(\mathrm{GL}_{{\zeta_{k}}}(\mathbb{C}) \times \mathrm{GL}_{q_{\zeta_{k}}}(\mathbb{C})\right) \longrightarrow \mathbb{C}^{*} \\
\left(A_{\eta_{1}}, B_{\eta_{1}}, \cdots, A_{\eta_{\alpha}}, B_{\eta_{\alpha}} ; C_{\theta_{1}}, D_{\theta_{1}}, \cdots, C_{\theta_{\beta}}, D_{\theta_{\beta}} ; E_{\zeta_{1}}, F_{\zeta_{1}}, \cdots, E_{\zeta_{\gamma}}, F_{\zeta_{\gamma}}\right) \\
\longmapsto \\
\prod_{i=1}^{\alpha}\left(\operatorname{det} A_{\eta_{i}}^{\eta_{i} / 2} \operatorname{det} B_{\eta_{i}}^{\eta_{i} / 2}\right) \prod_{j=1}^{\beta}\left(\operatorname{det} C_{\theta_{j}}^{\frac{\theta_{j}-1}{2}} \operatorname{det} D_{\theta_{j}{ }^{\frac{\theta_{j}+1}{2}}}\right) \prod_{k=1}^{\gamma}\left(\operatorname{det} E_{\zeta_{k}}^{\frac{\zeta_{k}+1}{2}} \operatorname{det} F_{\zeta_{k}}^{\frac{\zeta_{k}-1}{2}}\right) .
\end{aligned}
$$

Let $\Lambda_{\mathcal{H}}: \operatorname{End}_{\mathbb{C}} \mathbb{C}^{n} \longrightarrow \mathrm{M}_{n}(\mathbb{C})$ be the isomorphism of $\mathbb{C}$-algebras induced by the ordered basis $\mathcal{H}$ defined in (4.26). Let $M$ be the maximal compact subgroup of $\mathrm{SU}(p, q)$ which leaves invariant simultaneously the two subspace spanned by $\mathcal{H}_{+}$and $\mathcal{H}_{-}$. Clearly, $\Lambda_{\mathcal{H}}(M)=\mathrm{S}(\mathrm{U}(p) \times \mathrm{U}(q))$.

Proposition 4.14. Let $X \in \mathcal{N}_{\mathfrak{s u}(p, q)}, \Psi_{\mathrm{SU}(p, q)}\left(\mathcal{O}_{X}\right)=\left(\mathbf{d}, \operatorname{sgn}_{\mathcal{O}_{X}}\right)$. Let $\alpha:=\# \mathbb{E}_{\mathbf{d}}, \beta:=\# \mathbb{O}_{\mathbf{d}}^{1}$ and $\gamma:=\# \mathbb{O}_{\mathbf{d}}^{3}$. Let $\{X, H, Y\}$ be a $\mathfrak{s l}_{2}(\mathbb{R})$-triple in $\mathfrak{s u}(p, q)$ and $\left(p_{d}, q_{d}\right)$ the signature of the form $(\cdot, \cdot)_{d}$, $d \in \mathbb{N}_{\mathbf{d}}$, as defined in (4.6). Let $K$ be the maximal compact subgroup of $\mathcal{Z}_{\mathrm{SU}(p, q)}(X, H, Y)$ as in Lemma 4.13. Then $\Lambda_{\mathcal{H}}(K) \subset \mathrm{S}(\mathrm{U}(p) \times \mathrm{U}(q))$ is given by

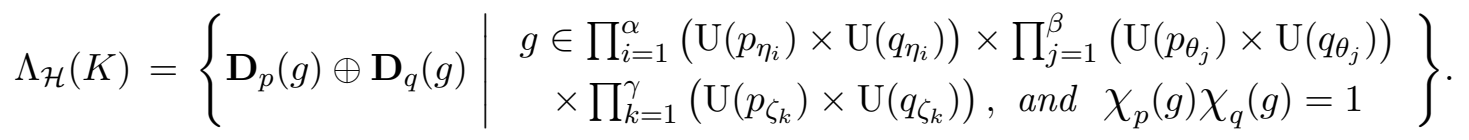

Proof. This follows by writing the matrices of the elements of the maximal compact subgroup $K$ in Lemma 4.13 with respect to the basis $\mathcal{H}$ as in (4.26).

Theorem 4.15. Let $X \in \mathfrak{s u}(p, q)$ be a nilpotent element. Let $\left(\mathbf{d}, \mathbf{s g n}_{\mathcal{O}_{X}}\right) \in \mathcal{Y}(p, q)$ be the signed Young diagram of the orbit $\mathcal{O}_{X}$ (that is, $\Psi_{\mathrm{SU}(p, q)}\left(\mathcal{O}_{X}\right)=\left(\mathbf{d}, \mathbf{s g n}_{\mathcal{O}_{X}}\right)$ as in the notation of Theorem 4.10). Let

$$
l:=\#\left\{d \in \mathbb{N}_{\mathbf{d}} \mid p_{d} \neq 0\right\}+\#\left\{d \in \mathbb{N}_{\mathbf{d}}, \mid q_{d} \neq 0\right\} .
$$

Then the following hold:

(1) If $\mathbb{N}_{\mathbf{d}}=\mathbb{E}_{\mathbf{d}}$, then $\operatorname{dim}_{\mathbb{R}} H^{2}\left(\mathcal{O}_{X}, \mathbb{R}\right)=l-1$.

(2) If $l=1$ and $\mathbb{N}_{\mathbf{d}}=\mathbb{O}_{\mathbf{d}}$, then $\operatorname{dim}_{\mathbb{R}} H^{2}\left(\mathcal{O}_{X}, \mathbb{R}\right)=0$.

(3) If $l \geq 2$ and $\# \mathbb{O}_{\mathbf{d}} \geq 1$, then $\operatorname{dim}_{\mathbb{R}} H^{2}\left(\mathcal{O}_{X}, \mathbb{R}\right)=l-2$.

Proof. This is clear when $X=0$. So assume that $X \neq 0$.

Let $\{X, H, Y\} \subset \mathfrak{s u}(p, q)$ be a $\mathfrak{s l}_{2}(\mathbb{R})$-triple. Let $K$ be the maximal compact subgroup of $\mathcal{Z}_{\mathrm{SU}(p, q)}(X, H, Y)$ as in Lemma 4.13 , and let $\mathcal{H}$ be as in (4.26). Let $M$ be the maximal compact subgroup of $\mathrm{SU}(p, q)$ which leaves invariant simultaneously the two subspace spanned by $\mathcal{H}_{+}$ and $\mathcal{H}_{-}$. Then $M$ contains $K$. It follows either from Proposition 4.14 or from Lemma 4.4 (4) that

$$
K \simeq K^{\prime}:=S\left(\prod_{d \in \mathbb{N}_{\mathbf{d}}}\left(\mathrm{U}\left(p_{d}\right) \times \mathrm{U}\left(q_{d}\right)\right)_{\Delta}^{d}\right) .
$$

This implies that $\operatorname{dim}_{\mathbb{R}} \mathfrak{z}(\mathfrak{k})=l-1$. We now appeal to Proposition 4.14 to make the following observations :

(1) If $\mathbb{N}_{\mathbf{d}}=\mathbb{E}_{\mathbf{d}}$, then $\mathfrak{k} \subset[\mathfrak{m}, \mathfrak{m}]$.

(2) If $\# \mathbb{O}_{\mathbf{d}} \geq 1$ and $l \geq 2$, then $\mathfrak{k}, \not \subset[\mathfrak{m}, \mathfrak{m}]$. 
Since $K$ is not necessarily connected, we need to show that the adjoint action of $K$ on $\mathfrak{z}(\mathfrak{k})$ is trivial. For this, first denote

$$
\mathbf{L}:=\prod_{d \in \mathbb{N}_{\mathbf{d}}}\left(\mathrm{U}\left(p_{d}\right) \times \mathrm{U}\left(q_{d}\right)\right)_{\Delta}^{d}
$$

and identify $K$ with $K^{\prime}$. Let $\mathfrak{l}$ be the Lie algebra of $\mathbf{L}$. Then

$$
[\mathbf{L}, \mathbf{L}]=\prod_{d \in \mathbb{N}_{\mathbf{d}}}\left(\mathrm{SU}\left(p_{d}\right) \times \mathrm{SU}\left(q_{d}\right)\right)_{\Delta}^{d} .
$$

In particular $[\mathbf{L}, \mathbf{L}] \subset K \subset \mathbf{L}$. Thus $[\mathfrak{l}, \mathfrak{l}]=[\mathfrak{k}, \mathfrak{k}]$, and hence $\mathfrak{z}(\mathfrak{k})=\mathfrak{k} \cap \mathfrak{z}(\mathfrak{l})$. Since $\mathbf{L}$ is connected, the adjoint action of $\mathbf{L}$ is trivial on $\mathfrak{z}(\mathfrak{l})$. So the adjoint action of $K$ on $\mathfrak{z}(\mathfrak{k})$ is trivial.

Proof of (1): From the above observations it follow that $\mathfrak{k} \subset[\mathfrak{m}, \mathfrak{m}]$ when $\mathbb{N}_{\mathbf{d}}=\mathbb{E}_{\mathbf{d}}$. As the adjoint action of $K$ on $\mathfrak{z}(\mathfrak{k})$ is trivial, we have $[(\mathfrak{z}(\mathfrak{k}) \cap[\mathfrak{m}, \mathfrak{m}])]^{K / K^{\circ}}=\mathfrak{z}(\mathfrak{k}) \cap[\mathfrak{m}, \mathfrak{m}]=\mathfrak{z}(\mathfrak{k})$. In view of Theorem 4.2 we now have $\operatorname{dim}_{\mathbb{R}} H^{2}\left(\mathcal{O}_{X}, \mathbb{R}\right)=l-1$.

Proof of (2): Suppose $\mathbf{d}=\left[d^{t_{d}}\right]$ where $t_{d} d=p+q$. Since $l=1$, it follows that either $p_{d}=t_{d}$ or $q_{d}=t_{d}$. In both cases we have $K \simeq S\left(\mathrm{U}\left(t_{d}\right)_{\Delta}^{d}\right)$. So $\mathfrak{z}(\mathfrak{k})$ is trivial. Hence, in view of Theorem 4.2 we have $\operatorname{dim}_{\mathbb{R}} H^{2}\left(\mathcal{O}_{X}, \mathbb{R}\right)=0$.

Proof of (3): From the above observations we have $\mathfrak{z}(\mathfrak{k}) \not \subset[\mathfrak{m}, \mathfrak{m}]$ when $\# \mathbb{O}_{\mathbf{d}} \geq 1$ and $l \geq 2$. Since $\operatorname{dim}_{\mathbb{R}} \mathfrak{z}(\mathfrak{m})=1$, it follows that $\operatorname{dim}_{\mathbb{R}}(\mathfrak{z}(\mathfrak{k}) \cap[\mathfrak{m}, \mathfrak{m}])=\operatorname{dim}_{\mathbb{R}} \mathfrak{z}(\mathfrak{k})-1$. By Theorem 4.2, and the fact that the adjoint action of $K$ on $\mathfrak{z}(\mathfrak{k})$ is trivial, we conclude that

$$
\operatorname{dim}_{\mathbb{R}} H^{2}\left(\mathcal{O}_{X}, \mathbb{R}\right)=\operatorname{dim}_{\mathbb{R}}\left[(\mathfrak{z}(\mathfrak{k}) \cap[\mathfrak{m}, \mathfrak{m}])^{*}\right]^{K / K^{\circ}}=l-2 .
$$

This completes the proof of the theorem.

4.5. Second cohomology groups of nilpotent orbits in $\mathfrak{s o}(p, q)$. Let $n$ be a positive integer and $(p, q)$ be a pair of non-negative integers such that $p+q=n$. The aim in this subsection is to compute the second cohomology groups of nilpotent orbits in the simple Lie algebra $\mathfrak{s o}(p, q)$ under the adjoint action of $\mathrm{SO}(p, q)^{\circ}$. We will further assume that $p, q>0$ as we deal with noncompact groups. Throughout this subsection $\langle\cdot, \cdot\rangle$ denotes the symmetric form on $\mathbb{R}^{n}$ defined by $\langle x, y\rangle:=x^{t} \mathrm{I}_{p, q} y, x, y \in \mathbb{R}^{n}$, where $\mathrm{I}_{p, q}$ is as in (2.1). In this subsection we will follow notation as defined in $\S 2$.

We first need to describe a suitable parametrization of $\mathcal{N}\left(\mathrm{SO}(p, q)^{\circ}\right)$, the set of all nilpotent orbits in $\mathfrak{s o}(p, q)$ under the adjoint action of $\mathrm{SO}(p, q)^{\circ}$. Let $\Psi_{\mathrm{SL}_{n}(\mathbb{R})}: \mathcal{N}\left(\mathrm{SL}_{n}(\mathbb{R})\right) \longrightarrow \mathcal{P}(n)$ be the parametrization of $\mathcal{N}\left(\mathrm{SL}_{n}(\mathbb{R})\right)$ as in Theorem 4.6. Since $\mathrm{SO}(p, q) \subset \mathrm{SL}_{n}(\mathbb{R})$ (consequently as, the set of nilpotent elements $\left.\mathcal{N}_{\mathfrak{s o}(p, q)} \subset \mathcal{N}_{\mathfrak{s l}(\mathbb{R})}\right)$ we have the inclusion map $\Theta_{\mathrm{SO}(p, q)^{\circ}}$ : $\mathcal{N}\left(\mathrm{SO}(p, q)^{\circ}\right) \longrightarrow \mathcal{N}\left(\mathrm{SL}_{n}(\mathbb{R})\right)$. Let

$$
\Psi_{\mathrm{SO}(p, q)^{\circ}}^{\prime}:=\Psi_{\mathrm{SL}_{n}(\mathbb{R})} \circ \Theta_{\mathrm{SO}(p, q)^{\circ}}: \mathcal{N}\left(\mathrm{SO}(p, q)^{\circ}\right) \longrightarrow \mathcal{P}(n)
$$

be the composition. Recall that $\Psi_{\mathrm{SO}(p, q)^{\circ}}^{\prime}\left(\mathcal{N}\left(\mathrm{SO}(p, q)^{\circ}\right)\right) \subset \mathcal{P}_{1}(n)$ (this follows form the first paragraph of Remark 5.16). Let $X \in \mathfrak{s o}(p, q)$ be a non-zero nilpotent element and $\mathcal{O}_{X}$ the corresponding nilpotent orbit in $\mathfrak{s o}(p, q)$ under the adjoint action of $\operatorname{SO}(p, q)^{\circ}$. Let $\{X, H, Y\} \subset$ $\mathfrak{s o}(p, q)$ be a $\mathfrak{s l}_{2}(\mathbb{R})$-triple. We now apply Proposition 5.14, Remark 5.16 (1), and follow the notation used therein. Let $V:=\mathbb{R}^{n}$ be the right $\mathbb{R}$-vector space of column vectors. Let $\left\{d_{1}, \cdots, d_{s}\right\}$, with $d_{1}<\cdots<d_{s}$, be ordered finite set of natural numbers that occur as dimension of non-zero irreducible $\operatorname{Span}_{\mathbb{R}}\{X, H, Y\}$-submodules of $V$. Recall that $M(d-1)$ is defined to be the isotypical component of $V$ containing all irreducible $\operatorname{Span}_{\mathbb{R}}\{X, H, Y\}$-submodules of $V$ with highest weight $d-1$ and as in $(2.2)$ we set $L(d-1):=V_{Y, 0} \cap M(d-1)$. Let $t_{d_{r}}:=\operatorname{dim}_{\mathbb{R}} L\left(d_{r}-1\right)$, $1 \leq r \leq s$. Then $\mathbf{d}:=\left[d_{1}^{t_{d_{1}}}, \cdots, d_{s}^{t_{d_{s}}}\right] \in \mathcal{P}_{1}(n)$, and moreover, $\Psi_{\mathrm{SO}(p, q)^{\circ}}^{\prime}\left(\mathcal{O}_{X}\right)=\mathbf{d}$. 
We next assign $\mathbf{s g n}_{\mathcal{O}_{X}} \in \mathcal{S}_{\mathbf{d}}^{\text {even }}(p, q)$ to each $\mathcal{O}_{X} \in \mathcal{N}\left(\operatorname{SO}(p, q)^{\circ}\right)$; see (2.8) for the definition of $\mathcal{S}_{\mathbf{d}}^{\text {even }}(p, q)$. For each $d \in \mathbb{N}_{\mathbf{d}}$ (see $(2.3)$ for the definition of $\mathbb{N}_{\mathbf{d}}$ ) we will define a $t_{d} \times d$ matrix $\left(m_{i j}^{d}\right)$ in $\mathbf{A}_{d}$ that depends only on the orbit $\mathcal{O}_{X}$; see (2.5) for the definition of $\mathbf{A}_{d}$. For this, recall that the form $(\cdot, \cdot)_{d}: L(d-1) \times L(d-1) \longrightarrow \mathbb{R}$, defined in (4.6), is symmetric or symplectic according as $d$ is odd or even. Denoting the signature of $(\cdot, \cdot)_{d}$ by $\left(p_{d}, q_{d}\right)$ when $d \in \mathbb{O}_{\mathbf{d}}$, we now define

$$
\begin{aligned}
& m_{i 1}^{\eta}:=+1 \quad \text { if } 1 \leq i \leq t_{\eta}, \quad \eta \in \mathbb{E}_{\mathbf{d}} ; \\
& m_{i 1}^{\theta}:=\left\{\begin{array}{ll}
+1 & \text { if } 1 \leq i \leq p_{\theta} \\
-1 & \text { if } p_{\theta}<i \leq t_{\theta}
\end{array}, \theta \in \mathbb{O}_{\mathbf{d}} ;\right.
\end{aligned}
$$

and for $j>1$, define $\left(m_{i j}^{d}\right)$ as in (4.15) and (4.16). Then the matrices $\left(m_{i j}^{d}\right)$ clearly verify $(\mathbf{Y d} .2)$. Set $\mathbf{s g n}_{\mathcal{O}_{X}}:=\left(\left(m_{i j}^{d_{1}}\right), \cdots,\left(m_{i j}^{d_{s}}\right)\right)$. It now follows from the last paragraph of Remark 5.21 and the above definition of $m_{i 1}^{\eta}$ for $\eta \in \mathbb{E}_{\mathbf{d}}$ that $\operatorname{sgn}_{\mathcal{O}_{X}} \in \mathcal{S}_{\mathbf{d}}^{\text {even }}(p, q)$. Thus we have the map

$$
\Psi_{\mathrm{SO}(p, q)^{\circ}}: \mathcal{N}\left(\mathrm{SO}(p, q)^{\circ}\right) \longrightarrow \mathcal{Y}_{1}^{\text {even }}(p, q), \mathcal{O}_{X} \longmapsto\left(\Psi_{\mathrm{SO}(p, q)^{\circ}}^{\prime}\left(\mathcal{O}_{X}\right), \operatorname{sgn}_{\mathcal{O}_{X}}\right) ;
$$

where $\mathcal{Y}_{1}^{\text {even }}(p, q)$ is as in $(2.11)$. The map $\Psi_{\mathrm{SO}(p, q)^{\circ}}$ is surjective.

Theorem 4.16. For the above map $\Psi_{\mathrm{SO}(p, q)^{\circ}}$,

$$
\# \Psi_{\mathrm{SO}(p, q)^{\circ}}^{-1}(\mathbf{d}, \mathbf{s g n})= \begin{cases}4 & \text { for all } \mathbf{d} \in \mathcal{P}_{\mathrm{v} . \text { even }}(n) \\ 2 & \text { for all } \mathbf{d} \in \mathcal{P}_{1}(n) \backslash \mathcal{P}_{\mathrm{v} . \text { even }}(n), \text { sgn } \in \mathcal{S}_{\mathbf{d}}^{\prime}(p, q) \\ 1 & \text { otherwise. }\end{cases}
$$

Remark 4.17. Taking into account the error in [CoMc, Lemma 9.3.1] pointed out in Remark 5.21 , the above parametrization in Theorem 4.16 is a modification of Theorem 9.3.4 in [CoMc].

Let $0 \neq X \in \mathcal{N}_{\mathfrak{s o}(p, q)}$ and $\{X, H, Y\} \subset \mathfrak{s o}(p, q)$ be a $\mathfrak{s l}_{2}(\mathbb{R})$-triple. Let $\Psi_{\mathrm{SO}(p, q)^{\circ}}\left(\mathcal{O}_{X}\right)=$ $\left(\mathbf{d}, \operatorname{sgn}_{\mathcal{O}_{X}}\right)$. Then $\Psi_{\mathrm{SO}(p, q)^{\circ}}^{\prime}\left(\mathcal{O}_{X}\right)=\mathbf{d}$. Recall that $\operatorname{sgn}_{\mathcal{O}_{X}}$ determines the signature of $(\cdot, \cdot)_{\theta}$ on $L(\theta-1), \theta \in \mathbb{O}_{\mathbf{d}}$; let $\left(p_{\theta}, q_{\theta}\right)$ be the signature of $(\cdot, \cdot)_{\theta}$.

First assume that $\mathbb{N}_{\mathbf{d}}=\mathbb{O}_{\mathbf{d}}$. Let $\left(v_{1}^{\theta}, \cdots, v_{t_{\theta}}^{\theta}\right)$ be an ordered $\mathbb{R}$-basis of $L(\theta-1)$ as in Proposition 5.14. It now follows from Proposition 5.14(3)(b) that $\left(v_{1}^{\theta}, \cdots, v_{t_{\theta}}^{\theta}\right)$ is an orthogonal basis for $(\cdot, \cdot)_{\theta}$ when $\theta \in \mathbb{O}_{\mathbf{d}}$. We also assume that the vectors in the ordered basis $\left(v_{1}^{\theta}, \cdots, v_{t_{\theta}}^{\theta}\right)$ satisfies the properties in Remark 5.16(1). In view of the signature of $(\cdot, \cdot)_{\theta}, \theta \in \mathbb{O}_{\mathbf{d}}$, we may further assume that

$$
\left(v_{j}^{\theta}, v_{j}^{\theta}\right)_{\theta}= \begin{cases}+1 & \text { if } 1 \leq j \leq p_{\theta} \\ -1 & \text { if } p_{\theta}<j \leq t_{\theta}\end{cases}
$$

For $\theta \in \mathbb{O}_{\mathbf{d}}$, let $\left\{w_{j l}^{\theta} \mid 1 \leq j \leq t_{\theta}, 0 \leq l \leq \theta-1\right\}$ be the $\mathbb{R}$-basis of $M(\theta-1)$ as in Lemma 5.17. For each $0 \leq l \leq \theta-1$, define

$$
V^{l}(\theta):=\operatorname{Span}_{\mathbb{R}}\left\{w_{1 l}^{\theta}, \cdots, w_{t_{\theta} l}^{\theta}\right\} .
$$

The ordered basis $\left(w_{1 l}^{\theta}, \cdots, w_{t_{\theta} l}^{\theta}\right)$ of $V^{l}(\theta)$ is denoted by $\mathcal{C}^{l}(\theta)$.

Lemma 4.18. For $\mathbb{N}_{\mathrm{d}}=\mathbb{O}_{\mathrm{d}}$,

$$
\mathcal{Z}_{\mathrm{SO}(p, q)}(X, H, Y)=\left\{g \in \mathrm{SO}(p, q) \mid \begin{array}{c}
g\left(V^{l}(\theta)\right) \subset V^{l}(\theta) \text { and } \\
{\left[\left.g\right|_{V^{l}(\theta)}\right]_{\mathcal{C}^{l}(\theta)}=\left[\left.g\right|_{V^{0}(\theta)}\right]_{\mathcal{C}^{0}(\theta)} \text { for all } \theta \in \mathbb{O}_{\mathbf{d}}, 0 \leq l<\theta}
\end{array}\right\} .
$$

Proof. We omit the proof as it is identical to that of Lemma 4.12. 
We next impose orderings on the sets $\left\{v \in \mathcal{C}^{l}(\theta) \mid\langle v, v\rangle>0\right\},\left\{v \in \mathcal{C}^{l}(\theta) \mid\langle v, v\rangle<0\right\}$. Define the ordered sets by $\mathcal{C}_{+}^{l}(\theta), \mathcal{C}_{-}^{l}(\theta), \mathcal{C}_{+}^{l}(\zeta)$ and $\mathcal{C}_{-}^{l}(\zeta)$ as in (4.19), (4.20), (4.21), (4.22), respectively according as $\theta \in \mathbb{O}_{\mathbf{d}}^{1}$ or $\zeta \in \mathbb{O}_{\mathbf{d}}^{3}$. For all $\theta \in \mathbb{O}_{\mathbf{d}}$ and $0 \leq l \leq \theta-1$, set

$$
V_{+}^{l}(\theta):=\operatorname{Span}_{\mathbb{R}}\left\{v \mid v \in \mathcal{C}^{l}(\theta),\langle v, v\rangle>0\right\}, \quad V_{-}^{l}(\theta):=\operatorname{Span}_{\mathbb{R}}\left\{v \mid v \in \mathcal{C}^{l}(\theta),\langle v, v\rangle<0\right\} .
$$

It is straightforward from (4.27), and the orthogonality relations in Lemma 5.17, that $\mathcal{C}_{+}^{l}(\theta)$ and $\mathcal{C}_{-}^{l}(\theta)$ are indeed ordered bases of $V_{+}^{l}(\theta)$ and $V_{-}^{l}(\theta)$, respectively.

In the next lemma we specify a maximal compact subgroup of $\mathcal{Z}_{\mathrm{SO}(p, q)}(X, H, Y)$ which will be used in Proposition 4.21. As before, the notation $(-1)^{l}$ stands for the sign ' + ' or the sign '-' according as $l$ is an even or odd integer.

Lemma 4.19. Suppose that $\mathbb{N}_{\mathbf{d}}=\mathbb{O}_{\mathbf{d}}$. Let $K$ be the subgroup of $\mathcal{Z}_{\mathrm{SO}(p, q)}(X, H, Y)$ consisting of all $g \in \mathcal{Z}_{\mathrm{SO}(p, q)}(X, H, Y)$ such that the following hold:

(1) $g\left(V_{+}^{l}(\theta)\right) \subset V_{+}^{l}(\theta)$ and $g\left(V_{-}^{l}(\theta)\right) \subset V_{-}^{l}(\theta)$, for all $\theta \in \mathbb{O}_{\mathbf{d}}$ and $0 \leq l \leq \theta-1$.

(2) When $\theta \in \mathbb{O}_{\mathbf{d}}^{1}$,

$$
\begin{aligned}
{\left[\left.g\right|_{V_{+}^{0}(\theta)}\right]_{\mathcal{C}_{+}^{0}(\theta)}= \begin{cases}{\left[\left.g\right|_{V_{(-1)^{l}}^{l}(\theta)}\right]_{\mathcal{C}_{(-1) l}^{l}}(\theta)} & \text { for all } 0 \leq l<(\theta-1) / 2 \\
{\left[\left.g\right|_{V_{+}^{(\theta-1) / 2}(\theta)}\right]_{\mathcal{C}_{+}^{(\theta-1) / 2}(\theta)}} & \text { for all }(\theta-1) / 2<l \leq \theta-1, \\
{\left[\left.g\right|_{V_{(-1)^{l+1}}^{l}(\theta)}\right]_{\mathcal{C}_{(-1)^{l+1}}^{l}(\theta)}} & \text { for all } 0 \leq l<(\theta-1) / 2\end{cases} } \\
{\left[\left.g\right|_{V_{-}^{0}(\theta)}\right]_{\mathcal{C}_{-}^{0}(\theta)}= \begin{cases}{\left[\left.g\right|_{V_{(-1)^{l+1}}^{l}(\theta)}\right]_{\mathcal{C}_{(-1)^{l+1}}^{l}(\theta)}} & \\
{\left[\left.g\right|_{V_{-}^{(\theta-1) / 2}(\theta)}\right]_{\mathcal{C}_{-}^{(\theta-1) / 2}(\theta)}} & \text { for all }(\theta-1) / 2<l \leq \theta-1 . \\
{\left[\left.g\right|_{V_{(-1)^{l}}^{l}(\theta)}\right]_{\mathcal{C}_{(-1)^{l}}^{l}}(\theta)} & \end{cases} }
\end{aligned}
$$

(3) When $\zeta \in \mathbb{O}_{\mathbf{d}}^{3}$,

$$
\begin{aligned}
& {\left[\left.g\right|_{V_{+}^{0}(\zeta)}\right]_{\mathcal{C}_{+}^{0}(\zeta)}= \begin{cases}{\left[\left.g\right|_{V_{(-1)^{l}}^{l}(\zeta)}\right]_{\mathcal{C}_{(-1)^{l}}^{l}(\zeta)}} & \text { for all } 0 \leq l<(\zeta-1) / 2 \\
{\left[\left.g\right|_{V_{-}^{(\zeta-1) / 2}(\zeta)}\right]_{\mathcal{C}_{-}^{(\zeta-1) / 2}(\zeta)}} & \text { for all }(\zeta-1) / 2<l \leq \zeta-1, \\
{\left[\left.g\right|_{V_{(-1)^{l+1}}^{l}(\zeta)}\right]_{\mathcal{C}_{(-1)^{l+1}}^{l}(\zeta)}} & \text { for all } 0 \leq l<(\zeta-1) / 2\end{cases} } \\
& {\left[\left.g\right|_{V_{-}^{0}(\zeta)}\right]_{\mathcal{C}_{-}^{0}(\zeta)}= \begin{cases}{\left[\left.g\right|_{V_{(-1)^{l+1}}^{l}(\zeta)}\right]_{\mathcal{C}_{(-1)^{l+1}}^{l}(\zeta)}} & \\
{\left[\left.g\right|_{V_{+}^{(\zeta-1) / 2}(\zeta)}\right]_{\mathcal{C}_{+}^{(\zeta-1) / 2}(\zeta)}} & \text { for all }(\zeta-1) / 2<l \leq \zeta-1 . \\
{\left[\left.g\right|_{V_{(-1)^{l}}^{l}(\zeta)}\right]_{\mathcal{C}_{(-1)^{l}}^{l}(\zeta)}} & \end{cases} }
\end{aligned}
$$

Then $K$ is a maximal compact subgroup of $\mathcal{Z}_{\mathrm{SO}(p, q)}(X, H, Y)$.

Proof. We omit the proof as it is identical to the proof of Lemma 4.13. 
The following lemma is required in the proof of Theorem $4.26(2)(\mathrm{iv})$. Recall that $\mathcal{B}^{0}(d)$ is an ordered basis of $L(d-1)$ as in (4.3) with $l=0$ and satisfying Remark 5.16 (1).

Lemma 4.20. Suppose that $\Psi_{\mathrm{SO}(p, 2)^{\circ}}\left(\mathcal{O}_{X}\right)=\left(\left[1^{p-2}, 2^{2}\right],\left(\left(m_{i j}^{1}\right),\left(m_{i j}^{2}\right)\right)\right)$, where $\left(m_{i j}^{1}\right)$ and $\left(m_{i j}^{2}\right)$ are $(p-2) \times 1$ and $2 \times 2$ matrices, respectively, satisfying $m_{i 1}^{1}=+1$ with $1 \leq i \leq p-2$, $m_{i 1}^{2}=+1$ with $1 \leq i \leq 2$, and $\mathbf{Y d}$.2. Let $K$ be the subgroup of $\mathcal{Z}_{\mathrm{SO}(p, 2)}(X, H, Y)$ consisting of all $g \in \mathcal{Z}_{\mathrm{SO}(p, 2)}(X, H, Y)$ such that the following hold:

(1) $g(L(1)) \subset L(1), g(X L(1)) \subset X L(1),\left[\left.g\right|_{L(1)}\right]_{\mathcal{B}^{0}(2)}=\left[\left.g\right|_{X L(1)}\right]_{\mathcal{B}^{1}(2)}$ and

$$
\left[\left.g\right|_{L(1)}\right]_{\mathcal{B}^{0}(2)}\left(\begin{array}{cc}
0 & -1 \\
1 & 0
\end{array}\right)=\left(\begin{array}{cc}
0 & -1 \\
1 & 0
\end{array}\right)\left[\left.g\right|_{L(1)}\right]_{\mathcal{B}^{0}(2)}
$$

(2) $g(L(0)) \subset L(0)$.

Then $K$ is a maximal compact subgroup of $\mathcal{Z}_{\mathrm{SO}(p, 2)}(X, H, Y)$.

Proof. Note that the form $(\cdot, \cdot)_{1}$ defined as in (4.6) is symmetric on $L(1-1) \times L(1-1)$ with signature $(p-2,0)$, and the form $(\cdot, \cdot)_{2}$ defined as in (4.6) is symplectic on $L(2-1) \times L(2-1)$. Moreover, it follows from Proposition 5.14 that $\mathcal{B}^{0}(2)=\left(v_{1}^{2} ; v_{2}^{2}\right)$ is a symplectic basis of $L(2-1)$ for $(\cdot, \cdot)_{2}$. Now the lemma follows from Lemma 4.4(4) and Lemma 4.34(1).

We next introduce some notation which will be needed in Proposition 4.21 and in Proposition 4.22. We assume that $\mathbb{N}_{\mathbf{d}}=\mathbb{O}_{\mathbf{d}}$. For $\theta \in \mathbb{O}_{\mathbf{d}}$, define

$$
\mathcal{C}_{+}(\theta):=\mathcal{C}_{+}^{0}(\theta) \vee \cdots \vee \mathcal{C}_{+}^{\theta-1}(\theta) \text { and } \mathcal{C}_{-}(\theta):=\mathcal{C}_{-}^{0}(\theta) \vee \cdots \vee \mathcal{C}_{-}^{\theta-1}(\theta) .
$$

Let $\beta:=\# \mathbb{O}_{\mathbf{d}}^{1}$ and $\gamma:=\# \mathbb{O}_{\mathbf{d}}^{3}$. We enumerate $\mathbb{O}_{\mathbf{d}}^{1}=\left\{\theta_{j} \mid 1 \leq j \leq \beta\right\}$ such that $\theta_{j}<\theta_{j+1}$ and similarly $\mathbb{O}_{\mathbf{d}}^{3}=\left\{\zeta_{j} \mid 1 \leq j \leq \gamma\right\}$ such that $\zeta_{j}<\zeta_{j+1}$. Set

$$
\begin{gathered}
\mathcal{O}_{+}^{1}:=\mathcal{C}_{+}\left(\theta_{1}\right) \vee \cdots \vee \mathcal{C}_{+}\left(\theta_{\beta}\right) ; \quad \mathcal{O}_{+}^{3}:=\mathcal{C}_{+}\left(\zeta_{1}\right) \vee \cdots \vee \mathcal{C}_{+}\left(\zeta_{\gamma}\right) ; \\
\mathcal{O}_{-}^{1}:=\mathcal{C}_{-}\left(\theta_{1}\right) \vee \cdots \vee \mathcal{C}_{-}\left(\theta_{\beta}\right) \quad \text { and } \quad \mathcal{O}_{-}^{3}:=\mathcal{C}_{-}\left(\zeta_{1}\right) \vee \cdots \vee \mathcal{C}_{-}\left(\zeta_{\gamma}\right) .
\end{gathered}
$$

Now define

$$
\mathcal{H}_{+}:=\mathcal{O}_{+}^{1} \vee \mathcal{O}_{+}^{3}, \quad \mathcal{H}_{-}:=\mathcal{O}_{-}^{1} \vee \mathcal{O}_{-}^{3} \text { and } \mathcal{H}:=\mathcal{H}_{+} \vee \mathcal{H}_{-} .
$$

It is clear that $\mathcal{H}$ is a standard orthogonal basis of $V$ such that $\mathcal{H}_{+}=\{v \in \mathcal{H} \mid\langle v, v\rangle=1\}$ and $\mathcal{H}_{-}=\{v \in \mathcal{H} \mid\langle v, v\rangle=-1\}$. In particular, $\# \mathcal{H}_{+}=p$ and $\# \mathcal{H}_{-}=q$. From the definition of $\mathcal{H}_{+}$and $\mathcal{H}_{-}$as given in (4.28) we have the following relations:

$$
\sum_{j=1}^{\beta}\left(\frac{\theta_{j}+1}{2} p_{\theta_{j}}+\frac{\theta_{j}-1}{2} q_{\theta_{j}}\right)+\sum_{k=1}^{\gamma}\left(\frac{\zeta_{k}-1}{2} p_{\zeta_{k}}+\frac{\zeta_{k}+1}{2} q_{\zeta_{k}}\right)=p
$$

and

$$
\sum_{j=1}^{\beta}\left(\frac{\theta_{j}-1}{2} p_{\theta_{j}}+\frac{\theta_{j}+1}{2} q_{\theta_{j}}\right)+\sum_{k=1}^{\gamma}\left(\frac{\zeta_{k}+1}{2} p_{\zeta_{k}}+\frac{\zeta_{k}-1}{2} q_{\zeta_{k}}\right)=q .
$$

The $\mathbb{R}$-algebra $\prod_{j=1}^{\beta}\left(\mathrm{M}_{p_{\theta_{j}}}(\mathbb{R}) \times \mathrm{M}_{q_{\theta_{j}}}(\mathbb{R})\right) \times \prod_{k=1}^{\gamma}\left(\mathrm{M}_{{\zeta_{k}}_{\zeta_{k}}}(\mathbb{R}) \times \mathrm{M}_{q_{\zeta_{k}}}(\mathbb{R})\right)$ is embedded in $\mathrm{M}_{p}(\mathbb{R})$ and in $\mathrm{M}_{q}(\mathbb{R})$ as follows:

$$
\begin{gathered}
\mathbf{D}_{p}: \prod_{j=1}^{\beta}\left(\mathrm{M}_{p_{\theta_{j}}}(\mathbb{R}) \times \mathrm{M}_{q_{\theta_{j}}}(\mathbb{R})\right) \times \prod_{k=1}^{\gamma}\left(\mathrm{M}_{p_{\zeta_{k}}}(\mathbb{R}) \times \mathrm{M}_{q_{\zeta_{k}}}(\mathbb{R})\right) \longrightarrow \mathrm{M}_{p}(\mathbb{R}) \\
\left(C_{\theta_{1}}, D_{\theta_{1}}, \cdots, C_{\theta_{\beta}}, D_{\theta_{\beta}} ; E_{\zeta_{1}}, F_{\zeta_{1}}, \cdots, E_{\zeta_{\gamma}}, F_{\zeta_{\gamma}}\right) \longmapsto
\end{gathered}
$$


$\bigoplus_{j=1}^{\beta}\left(\left(C_{\theta_{j}} \oplus D_{\theta_{j}}\right)^{\frac{\theta_{j}-1}{4}} \oplus C_{\theta_{j}} \oplus\left(C_{\theta_{j}} \oplus D_{\theta_{j}}\right)^{\frac{\theta_{j}-1}{4}}\right) \oplus \bigoplus_{k=1}^{\gamma}\left(\left(E_{\zeta_{k}} \oplus F_{\zeta_{k}}\right)^{\frac{\zeta_{k}+1}{4}} \oplus\left(F_{\zeta_{k}} \oplus E_{\zeta_{k}}\right)^{\frac{\zeta_{k}-3}{4}} \oplus F_{\zeta_{k}}\right)$

and

$$
\begin{gathered}
\mathbf{D}_{q}: \prod_{j=1}^{\beta}\left(\mathrm{M}_{p_{\theta_{j}}}(\mathbb{R}) \times \mathrm{M}_{q_{\theta_{j}}}(\mathbb{R})\right) \times \prod_{k=1}^{\gamma}\left(\mathrm{M}_{p_{\zeta_{k}}}(\mathbb{R}) \times \mathrm{M}_{q_{\zeta_{k}}}(\mathbb{R})\right) \longrightarrow \mathrm{M}_{q}(\mathbb{R}) \\
\left(C_{\theta_{1}}, D_{\theta_{1}}, \cdots, C_{\theta_{\beta}}, D_{\theta_{\beta}} ; E_{\zeta_{1}}, F_{\zeta_{1}}, \cdots, E_{\zeta_{\gamma}}, F_{\zeta_{\gamma}}\right) \longmapsto \\
\bigoplus_{j=1}^{\beta}\left(\left(D_{\theta_{j}} \oplus C_{\theta_{j}}\right)^{\frac{\theta_{j}-1}{4}} \oplus D_{\theta_{j}} \oplus\left(D_{\theta_{j}} \oplus C_{\theta_{j}}\right)^{\frac{\theta_{j}-1}{4}}\right) \oplus \bigoplus_{k=1}^{\gamma}\left(\left(F_{\zeta_{k}} \oplus E_{\zeta_{k}}\right)^{\frac{\zeta_{k}+1}{4}} \oplus\left(E_{\zeta_{k}} \oplus F_{\zeta_{k}}\right)^{\frac{\zeta_{k}-3}{4}} \oplus E_{\zeta_{k}}\right) .
\end{gathered}
$$

Define two characters

$$
\begin{aligned}
& \chi_{p}: \prod_{j=1}^{\beta}\left(\mathrm{O}_{p_{\theta_{j}}} \times \mathrm{O}_{q_{\theta_{j}}}\right) \times \prod_{k=1}^{\gamma}\left(\mathrm{O}_{p_{\zeta_{k}}} \times \mathrm{O}_{q_{\zeta_{k}}}\right) \longrightarrow \mathbb{R} \backslash\{0\} \\
& \left(C_{\theta_{1}}, D_{\theta_{1}}, \cdots, C_{\theta_{\beta}}, D_{\theta_{\beta}} ; E_{\zeta_{1}}, F_{\zeta_{1}}, \cdots, E_{\zeta_{\gamma}}, F_{\zeta_{\gamma}}\right) \longmapsto \\
& \prod_{j=1}^{\beta}\left(\operatorname{det} C_{\theta_{j}}^{\frac{\theta_{j}+1}{2}} \operatorname{det} D_{\theta_{j}}^{\frac{\theta_{j}-1}{2}}\right) \prod_{k=1}^{\gamma}\left(\operatorname{det} E_{\zeta_{k}}^{\frac{\zeta_{k}-1}{2}} \operatorname{det} F_{\zeta_{k}}^{\frac{\zeta_{k}+1}{2}}\right)=\prod_{j=1}^{\beta} \operatorname{det} C_{\theta_{j}} \prod_{k=1}^{\gamma} \operatorname{det} E_{\zeta_{k}}
\end{aligned}
$$

and

$$
\begin{gathered}
\chi_{q}: \prod_{j=1}^{\beta}\left(\mathrm{O}_{p_{\theta_{j}}} \times \mathrm{O}_{q_{\theta_{j}}}\right) \times \prod_{k=1}^{\gamma}\left(\mathrm{O}_{p_{\zeta_{k}}} \times \mathrm{O}_{q_{\zeta_{k}}}\right) \longrightarrow \mathbb{R} \backslash\{0\} \\
\left(C_{\theta_{1}}, D_{\theta_{1}}, \cdots, C_{\theta_{\beta}}, D_{\theta_{\beta}} ; E_{\zeta_{1}}, F_{\zeta_{1}}, \cdots, E_{\zeta_{\gamma}}, F_{\zeta_{\gamma}}\right) \longmapsto \\
\prod_{j=1}^{\beta}\left(\operatorname{det} C_{\theta_{i}}^{\frac{\theta_{i}-1}{2}} \operatorname{det} D_{\theta_{i}}^{\frac{\theta_{i}+1}{2}}\right) \prod_{k=1}^{\gamma}\left(\operatorname{det} E_{\zeta_{k}}^{\frac{\zeta_{k}+1}{2}} \operatorname{det} F_{\zeta_{k}}^{\frac{\zeta_{k}-1}{2}}\right)=\prod_{j=1}^{\beta} \operatorname{det} D_{\theta_{j}} \prod_{k=1}^{\gamma} \operatorname{det} F_{\zeta_{k}} .
\end{gathered}
$$

Let $\Lambda_{\mathcal{H}}: \operatorname{End}_{\mathbb{R}} \mathbb{R}^{n} \longrightarrow \mathrm{M}_{n}(\mathbb{R})$ be the isomorphism of $\mathbb{R}$-algebras induced by the ordered basis $\mathcal{H}$ in (4.28). Let $M$ be the maximal compact subgroup of $\mathrm{SO}(p, q)$ which leaves invariant simultaneously the two subspaces spanned by $\mathcal{H}_{+}$and $\mathcal{H}_{-}$. Clearly, $\Lambda_{\mathcal{H}}(M)=\mathrm{S}(\mathrm{O}(p) \times \mathrm{O}(q))$.

Proposition 4.21. Let $X \in \mathcal{N}_{\mathfrak{s o}(p, q)}, \Psi_{\mathrm{SO}(p, q)^{\circ}}\left(\mathcal{O}_{X}\right)=\left(\mathbf{d}, \mathbf{s g n}_{\mathcal{O}_{X}}\right)$. Assume that $\mathbb{N}_{\mathbf{d}}=\mathbb{O}_{\mathbf{d}}$. Let $\beta:=\# \mathbb{O}_{\mathbf{d}}^{1}$ and $\gamma:=\# \mathbb{O}_{\mathbf{d}}^{3}$. Let $\{X, H, Y\} \subset \mathfrak{s o}(p, q)$ be a $\mathfrak{s l}_{2}(\mathbb{R})$-triple, and let $\left(p_{\theta}, q_{\theta}\right)$ be the signature of the form $(\cdot, \cdot)_{\theta}$ for all $\theta \in \mathbb{O}_{\mathbf{d}}$ as defined in (4.6). Let $K$ be the maximal compact subgroup of $\mathcal{Z}_{\mathrm{SO}(p, q)}(X, H, Y)$ as in Lemma 4.19. Then $\Lambda_{\mathcal{H}}(K) \subset \mathrm{S}(\mathrm{O}(p) \times \mathrm{O}(q))$ is given by

$$
\Lambda_{\mathcal{H}}(K)=\left\{\mathbf{D}_{p}(g) \oplus \mathbf{D}_{q}(g) \mid \begin{array}{c}
g \in \prod_{j=1}^{\beta}\left(\mathrm{O}_{p_{\theta_{j}}} \times \mathrm{O}_{q_{\theta_{j}}}\right) \times \prod_{k=1}^{\gamma}\left(\mathrm{O}_{p_{\zeta_{k}}} \times \mathrm{O}_{q_{\zeta_{k}}}\right) \\
\text { and } \chi_{p}(g) \chi_{q}(g)=1
\end{array}\right\} .
$$

Proof. This follows by writing the matrices of the elements of the maximal compact subgroup $K$ in Lemma 4.19 with respect to the basis $\mathcal{H}$ as in (4.28).

As the subgroup $\mathrm{SO}(p, q)^{\circ}$ is normal in $\mathrm{SO}(p, q)$, so is $\mathcal{Z}_{\mathrm{SO}(p, q)^{\circ}}(X, H, Y)$ in $\mathcal{Z}_{\mathrm{SO}(p, q)}(X, H, Y)$. In particular, as $K$ is a maximal compact subgroup in $\mathcal{Z}_{\mathrm{SO}(p, q)}(X, H, Y)$, it follows that

$$
K_{\mathbb{O}}:=K \cap \mathcal{Z}_{\mathrm{SO}(p, q)^{\circ}}(X, H, Y)=K \cap \mathrm{SO}(p, q)^{\circ}
$$

is a maximal compact subgroup of $\mathcal{Z}_{\mathrm{SO}(p, q)^{\circ}}(X, H, Y)$. The next proposition gives an explicit description of $\Lambda_{\mathcal{H}}\left(K_{\mathbb{O}}\right)$ in $\mathrm{SO}(p) \times \mathrm{SO}(q)$. 
Proposition 4.22. Let $X \in \mathcal{N}_{\mathfrak{s o}(p, q)}, \Psi_{\mathrm{SO}(p, q)^{\circ}}\left(\mathcal{O}_{X}\right)=\left(\mathbf{d}, \operatorname{sgn}_{\mathcal{O}_{X}}\right)$. We assume that $\mathbb{N}_{\mathbf{d}}=\mathbb{O}_{\mathbf{d}}$. Let $\beta:=\# \mathbb{O}_{\mathbf{d}}^{1}$ and $\gamma:=\# \mathbb{O}_{\mathbf{d}}^{3}$. Let $\{X, H, Y\} \subset \mathfrak{s o}(p, q)$ be $a \mathfrak{s l}_{2}(\mathbb{R})$-triple, and let $\left(p_{\theta}, q_{\theta}\right)$ be the signature of the form $(\cdot, \cdot)_{\theta}$ for all $\theta \in \mathbb{O}_{\mathbf{d}}$ as defined in (4.6). Let $K_{\mathbb{O}}$ be the maximal compact subgroup of $\mathcal{Z}_{\mathrm{SO}(p, q)^{\circ}}(X, H, Y)$ as in the preceding paragraph. Then $\Lambda_{\mathcal{H}}\left(K_{\mathbb{O}}\right) \subset \mathrm{SO}(p) \times \mathrm{SO}(q)$ is given by

$$
\left\{\mathbf{D}_{p}(g) \oplus \mathbf{D}_{q}(g) \mid \begin{array}{c}
g \in \prod_{j=1}^{\beta}\left(\mathrm{O}_{p_{\theta_{j}}} \times \mathrm{O}_{q_{\theta_{j}}}\right) \times \prod_{k=1}^{\gamma}\left(\mathrm{O}_{p_{\zeta_{k}}} \times \mathrm{O}_{q_{\zeta_{k}}}\right) \\
\text { and } \chi_{p}(g)=1, \chi_{q}(g)=1
\end{array}\right\} .
$$

Moreover, the above group is isomorphic to

$$
S\left(\prod_{j=1}^{\beta} \mathrm{O}_{p_{\theta_{j}}} \times \prod_{k=1}^{\gamma} \mathrm{O}_{p_{\zeta_{k}}}\right) \times S\left(\prod_{j=1}^{\beta} \mathrm{O}_{q_{\theta_{j}}} \times \prod_{k=1}^{\gamma} \mathrm{O}_{q_{\zeta_{k}}}\right) .
$$

Proof. Let $V_{+}$and $V_{-}$be the $\mathbb{R}$-spans of $\mathcal{H}_{+}$and $\mathcal{H}_{-}$respectively. Let $M$ be the maximal compact subgroup in $\mathrm{SO}(p, q)$ which simultaneously leaves the subspaces $V_{+}$and $V_{-}$invariant. It is clear that $M^{\circ}$ is a maximal compact subgroup of $\mathrm{SO}(p, q)^{\circ}$. Hence

$$
M^{\circ}=\mathrm{SO}(p, q)^{\circ} \cap M=\left\{g \in \mathrm{SO}(p, q)|\operatorname{det} g|_{V_{+}}=1,\left.\operatorname{det} g\right|_{V_{-}}=1\right\} .
$$

As $K \subset M$, we have that $K \cap \mathrm{SO}(p, q)^{\circ}=K \cap M^{\circ}$. The proposition now follows.

Set

$\mathbb{O}_{\mathbf{d}}^{-}:=\left\{\theta \mid \theta \in \mathbb{O}_{\mathbf{d}},(\cdot, \cdot)_{\theta}\right.$ is negative definite $\}$ and $\mathbb{O}_{\mathbf{d}}^{+}:=\left\{\theta \mid \theta \in \mathbb{O}_{\mathbf{d}},(\cdot, \cdot)_{\theta}\right.$ is positive definite $\}$.

Lemma 4.23. Let $X \in \mathfrak{s o}(p, q)$ be a nilpotent element. Let $\left(\mathbf{d}, \mathbf{s g n}_{\mathcal{O}_{X}}\right) \in \mathcal{Y}_{1}^{\text {even }}(p, q)$ be the signed Young diagram of the orbit $\mathcal{O}_{X}$ (that is, $\Psi_{\mathrm{SO}(p, q)}{ }^{\circ}\left(\mathcal{O}_{X}\right)=\left(\mathbf{d}, \mathbf{s g n}_{\mathcal{O}_{X}}\right)$ as in the notation of Theorem 4.16). We moreover assume that $\mathbb{N}_{\mathbf{d}} \stackrel{=}{=} \mathbb{O}_{\mathbf{d}}$. Let $K_{\mathbb{O}}$ be the maximal compact

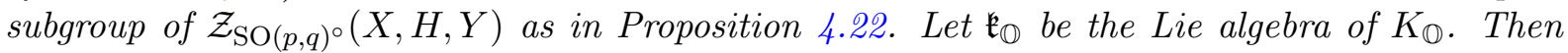
the following hold:

(1) If $\#\left(\mathbb{O}_{\mathbf{d}} \backslash \mathbb{O}_{\mathbf{d}}^{-}\right)=1, \#\left(\mathbb{O}_{\mathbf{d}} \backslash \mathbb{O}_{\mathbf{d}}^{+}\right)=1$ and $p_{\theta_{1}}=q_{\theta_{2}}=2$ for $\theta_{1} \in \mathbb{O}_{\mathbf{d}} \backslash \mathbb{O}_{\mathbf{d}}^{-}, \theta_{2} \in \mathbb{O}_{\mathbf{d}} \backslash \mathbb{O}_{\mathbf{d}}^{+}$, then $\operatorname{dim}_{\mathbb{R}}\left[\mathfrak{z}\left(\mathfrak{k}_{\mathbb{O}}\right)\right]^{K_{\mathbb{O}} / K_{\mathbb{O}}^{\circ}}=2$.

(2) Suppose that either $\#\left(\mathbb{O}_{\mathbf{d}} \backslash \mathbb{O}_{\mathbf{d}}^{-}\right)=1, p_{\theta_{1}}=2$ for $\theta_{1} \in \mathbb{O}_{\mathbf{d}} \backslash \mathbb{O}_{\mathbf{d}}^{-}$, or $\#\left(\mathbb{O}_{\mathbf{d}} \backslash \mathbb{O}_{\mathbf{d}}^{+}\right)=1$, $q_{\theta_{2}}=2$ for $\theta_{2} \in \mathbb{O}_{\mathbf{d}} \backslash \mathbb{O}_{\mathbf{d}}^{+}$. Moreover, suppose that both the conditions do not hold simultaneously. Then $\operatorname{dim}_{\mathbb{R}}\left[\mathfrak{z}\left(\mathfrak{k}_{\mathbb{O}}\right)\right]^{K_{\mathbb{O}} / K_{\mathbb{O}}^{\circ}}=1$.

(3) In all other cases, $\operatorname{dim}_{\mathbb{R}}\left[\mathfrak{z}\left(\mathfrak{k}_{\mathbb{O}}\right)\right]^{K_{\mathbb{O}} / K_{\mathbb{O}}^{\circ}}=0$.

Proof. In view of (4.11), (4.12) and Proposition 4.22, the lemma is clear.

Lemma 4.24. Let $W$ be a finite dimensional vector space over $\mathbb{R}$, and let $\langle\cdot, \cdot\rangle^{\prime}$ be a nondegenerate symmetric bilinear form on $W$. Let $W_{1}, W_{2} \subset W$ be subspaces such that $W_{1} \perp W_{2}$ and $W=W_{1} \oplus W_{2}$. Let $\langle\cdot, \cdot\rangle_{2}^{\prime}$ be the restriction of $\langle\cdot, \cdot\rangle^{\prime}$ to $W_{2}$. Then

$$
\begin{aligned}
& \operatorname{SO}\left(W,\langle\cdot, \cdot\rangle^{\prime}\right)^{\circ} \cap\left\{g \in \mathrm{SO}\left(W,\langle\cdot, \cdot\rangle^{\prime}\right)\left|g\left(W_{1}\right) \subset W_{1}, g\left(W_{2}\right) \subset W_{2}, g\right|_{W_{1}}=\operatorname{Id}_{W_{1}}\right\} \\
= & \left\{g \in \mathrm{SO}\left(W,\langle\cdot, \cdot\rangle^{\prime}\right)\left|g\left(W_{1}\right) \subset W_{1}, g\left(W_{2}\right) \subset W_{2}, g\right|_{W_{1}}=\operatorname{Id}_{W_{1}},\left.g\right|_{W_{2}} \in \operatorname{SO}\left(W_{2},\langle\cdot, \cdot\rangle_{2}^{\prime}\right)^{\circ}\right\} .
\end{aligned}
$$

In particular,

$$
\mathrm{SO}\left(W,\langle\cdot, \cdot\rangle^{\prime}\right)^{\circ} \cap\left\{g \in \mathrm{SO}\left(W,\langle\cdot, \cdot\rangle^{\prime}\right)\left|g\left(W_{1}\right) \subset W_{1}, g\left(W_{2}\right) \subset W_{2}, g\right|_{W_{1}}=\operatorname{Id}_{W_{1}}\right\}
$$

is isomorphic to $\mathrm{SO}\left(W_{2},\langle\cdot, \cdot\rangle_{2}^{\prime}\right)^{\circ}$. 
Proof. Let $\left(p_{2}, q_{2}\right)$ be the signature of $\langle\cdot, \cdot\rangle_{2}^{\prime}$. If either $p_{2}=0$ or $q_{2}=0$, then as $\operatorname{SO}\left(W_{2},\langle\cdot, \cdot\rangle_{2}^{\prime}\right)$ $=\mathrm{SO}\left(W_{2},\langle\cdot, \cdot\rangle_{2}^{\prime}\right)^{\circ}$ the lemma follows immediately.

Assumption that $p_{2}>0$ and $q_{2}>0$. In this case, considering an orthogonal basis of $W_{2}$ for the form $\langle\cdot, \cdot\rangle_{2}^{\prime}$ we easily construct a linear map $A: W \longrightarrow W$ such that $\left.A\right|_{W_{1}}=\operatorname{Id}_{W_{1}}$, $A\left(W_{2}\right) \subset W_{2},\left(\left.A\right|_{W_{2}}\right)^{2}=\operatorname{Id}_{W_{2}}$, and $\left.A\right|_{W_{2}} \in \mathrm{SO}\left(W_{2},\langle\cdot, \cdot\rangle_{2}^{\prime}\right) \backslash \mathrm{SO}\left(W_{2},\langle\cdot, \cdot\rangle_{2}^{\prime}\right)^{\circ}$. It is then clear that

$$
A \in \mathrm{SO}\left(W,\langle\cdot, \cdot\rangle^{\prime}\right) \backslash \mathrm{SO}\left(W,\langle\cdot, \cdot\rangle^{\prime}\right)^{\circ} .
$$

Let $\Gamma \subset \operatorname{GL}(W)$ be the subgroup generated by $A$ and $\Gamma^{\prime} \subset \operatorname{GL}\left(W_{2}\right)$ the subgroup generated by $\left.A\right|_{W_{2}}$. It then follows that $\mathrm{SO}\left(W,\langle\cdot, \cdot\rangle^{\prime}\right)=\Gamma \mathrm{SO}\left(W,\langle\cdot, \cdot\rangle^{\prime}\right)^{\circ}$ and $\mathrm{SO}\left(W_{2},\langle\cdot, \cdot\rangle_{2}^{\prime}\right)=$ $\Gamma^{\prime} \mathrm{SO}\left(W_{2},\langle\cdot, \cdot\rangle_{2}^{\prime}\right)^{\circ}$. Now the lemma follows.

We now describe the second cohomology groups of nilpotent orbits in $\mathfrak{s o}(p, q)$ when $p>0, q>$ 0 . As we will consider only simple Lie algebras, to ensure simplicity of $\mathfrak{s o}(p, q)$, in view of $[\mathrm{Kn}$, Theorem 6.105, p. 421] and isomorphisms (iv), (v), (vi), (ix), (x) in [He, Chapter X, §6, pp. $519-520]$, we need the additional restriction that $(p, q) \notin\{(1,1),(2,2)\}$.

Theorem 4.25. Let $p \neq 2, q \neq 2$ and $(p, q) \neq(1,1)$. Let $X \in \mathfrak{s o}(p, q)$ be a nilpotent element. Let $\left(\mathbf{d}, \mathbf{s g n}_{\mathcal{O}_{X}}\right) \in \mathcal{Y}_{1}^{\text {even }}(p, q)$ be the signed Young diagram of the orbit $\mathcal{O}_{X}$ (that is, $\Psi_{\mathrm{SO}(p, q)^{\circ}}\left(\mathcal{O}_{X}\right)=\left(\mathbf{d}, \mathbf{s g n}_{\mathcal{O}_{X}}\right)$ as in the notation of Theorem 4.16). Then the following hold:

(1) If $\#\left(\mathbb{O}_{\mathbf{d}} \backslash \mathbb{O}_{\mathbf{d}}^{-}\right)=1, \#\left(\mathbb{O}_{\mathbf{d}} \backslash \mathbb{O}_{\mathbf{d}}^{+}\right)=1$ and $p_{\theta_{1}}=q_{\theta_{2}}=2$ when $\theta_{1} \in \mathbb{O}_{\mathbf{d}} \backslash \mathbb{O}_{\mathbf{d}}^{-}$and $\theta_{2} \in \mathbb{O}_{\mathbf{d}} \backslash \mathbb{O}_{\mathbf{d}}^{+}$, then $\operatorname{dim}_{\mathbb{R}} H^{2}\left(\mathcal{O}_{X}, \mathbb{R}\right)=\left(\# \mathbb{E}_{\mathbf{d}}+2\right)$.

(2) Suppose that either $\#\left(\mathbb{O}_{\mathbf{d}} \backslash \mathbb{O}_{\mathbf{d}}^{-}\right)=1$ and $p_{\theta_{1}}=2$ for $\theta_{1} \in \mathbb{O}_{\mathbf{d}} \backslash \mathbb{O}_{\mathbf{d}}^{-}$, or $\#\left(\mathbb{O}_{\mathbf{d}} \backslash \mathbb{O}_{\mathbf{d}}^{+}\right)=1$ and $q_{\theta_{2}}=2$ for $\theta_{2} \in \mathbb{O}_{\mathbf{d}} \backslash \mathbb{O}_{\mathbf{d}}^{+}$. Moreover, suppose that the above two conditions do not hold simultaneously. Then $\operatorname{dim}_{\mathbb{R}} H^{2}\left(\mathcal{O}_{X}, \mathbb{R}\right)=\left(\# \mathbb{E}_{\mathbf{d}}+1\right)$.

(3) In all other cases $\operatorname{dim}_{\mathbb{R}} H^{2}\left(\mathcal{O}_{X}, \mathbb{R}\right)=\# \mathbb{E}_{\mathbf{d}}$.

Proof. Let $p+q=n$. As the theorem is evident when $X=0$, we assume that $X \neq 0$.

Let $\{X, H, Y\} \subset \mathfrak{s o}(p, q)$ be a $\mathfrak{s l}_{2}(\mathbb{R})$-triple. Let $V:=\mathbb{R}^{n}$ be the right $\mathbb{R}$-vector space of column vectors. We consider $V$ as a $\operatorname{Span}_{\mathbb{R}}\{X, H, Y\}$-module via its natural $\mathfrak{s o}(p, q)$-module structure. Let

$$
V_{\mathbb{E}}:=\bigoplus_{\eta \in \mathbb{E}_{\mathbf{d}}} M(\eta-1) ; \quad V_{\mathbb{O}}:=\bigoplus_{\theta \in \mathbb{O}_{\mathbf{d}}} M(\theta-1) .
$$

Using Lemma 5.12 it follows that $V=V_{\mathbb{E}} \oplus V_{\mathbb{O}}$ is an orthogonal decomposition of $V$ with respect to $\langle\cdot, \cdot\rangle$. Let $\langle\cdot, \cdot\rangle_{\mathbb{E}}:=\left.\langle\cdot, \cdot\rangle\right|_{V_{\mathbb{E}} \times V_{\mathbb{E}}}$ and $\langle\cdot, \cdot\rangle_{\mathbb{O}}:=\left.\langle\cdot, \cdot\rangle\right|_{V_{\mathbb{O}} \times V_{\mathbb{O}}}$. Let $X_{\mathbb{E}}:=\left.X\right|_{V_{\mathbb{E}}}, X_{\mathbb{O}}:=\left.X\right|_{V_{\mathbb{O}}}$, $H_{\mathbb{E}}:=\left.H\right|_{V_{\mathbb{E}}}, H_{\mathbb{O}}:=\left.H\right|_{V_{\mathbb{O}}}, Y_{\mathbb{E}}:=\left.Y\right|_{V_{\mathbb{E}}}$ and $Y_{\mathbb{O}}:=\left.Y\right|_{V_{\mathbb{O}}}$. Then we have the following natural isomorphism

$$
\mathcal{Z}_{\mathrm{SO}(p, q)}(X, H, Y) \simeq \mathcal{Z}_{\mathrm{SO}\left(V_{\mathbb{E}},\langle\cdot, \cdot\rangle_{\mathbb{E}}\right)}\left(X_{\mathbb{E}}, H_{\mathbb{E}}, Y_{\mathbb{E}}\right) \times \mathcal{Z}_{\mathrm{SO}\left(V_{\mathbb{O}},\langle\cdot, \cdot\rangle_{\mathbb{O}}\right)}\left(X_{\mathbb{O}}, H_{\mathbb{O}}, Y_{\mathbb{O}}\right)
$$

As, the form $(\cdot, \cdot)_{\eta}$ on $L(\eta-1)$ is non-degenerate and symplectic for all $\eta \in \mathbb{E}_{\mathbf{d}}$, it follows from Lemma 4.4 (4) that

$$
\mathcal{Z}_{\mathrm{SO}\left(V_{\mathbb{E}},\langle\cdot, \cdot\rangle_{\mathbb{E}}\right)}\left(X_{\mathbb{E}}, H_{\mathbb{E}}, Y_{\mathbb{E}}\right) \simeq \prod_{\eta \in \mathbb{E}_{\mathbf{d}}} \operatorname{Sp}\left(t_{\eta} / 2, \mathbb{R}\right)
$$

In particular, $\mathcal{Z}_{\mathrm{SO}\left(V_{\mathbb{E}},\langle\cdot, \cdot\rangle_{\mathbb{E}}\right)}\left(X_{\mathbb{E}}, H_{\mathbb{E}}, Y_{\mathbb{E}}\right)$ is connected, and hence using Lemma 4.24, (4.29) and (4.30) it follows that

$$
\mathcal{Z}_{\mathrm{SO}(p, q)^{\circ}}(X, H, Y) \simeq \mathcal{Z}_{\mathrm{SO}\left(V_{\mathbb{E}},\langle\cdot, \cdot\rangle_{\mathbb{E}}\right)}\left(X_{\mathbb{E}}, H_{\mathbb{E}}, Y_{\mathbb{E}}\right) \times \mathcal{Z}_{\mathrm{SO}\left(V_{\mathbb{O}},\langle\cdot, \cdot\rangle_{\mathbb{O}}\right)^{\circ}}\left(X_{\mathbb{O}}, H_{\mathbb{O}}, Y_{\mathbb{O}}\right) .
$$

Let $K_{\mathbb{E}}$ be a maximal compact subgroup of $\mathcal{Z}_{\mathrm{SO}\left(V_{\mathbb{E}},\langle\cdot, \cdot\rangle_{\mathbb{E}}\right)}\left(X_{\mathbb{E}}, H_{\mathbb{E}}, Y_{\mathbb{E}}\right) \simeq \prod_{\eta \in \mathbb{E}_{\mathbf{d}}} \operatorname{Sp}\left(t_{\eta} / 2, \mathbb{R}\right)$. Setting $\# \mathbb{O}_{\mathbf{d}}:=r$, enumerate $\mathbb{O}_{\mathbf{d}}=\left\{a_{1}, \cdots, a_{r}\right\}$ such that $a_{i}<a_{i+1}$ for all $i$. We next 
set $\mathbf{d}_{\mathbb{O}}:=\left[a_{1}^{t_{a_{1}}}, \cdots, a_{r}^{t_{a_{r}}}\right]$. As $\sum_{d \in \mathbb{O}_{\mathbf{d}}} t_{d} d=\operatorname{dim}_{\mathbb{R}} V_{\mathbb{O}}$, we have $\mathbf{d}_{\mathbb{O}} \in \mathcal{P}\left(\operatorname{dim}_{\mathbb{R}} V_{\mathbb{O}}\right)$. We recall that $K_{\mathbb{O}}:=K \cap \mathcal{Z}_{\mathrm{SO}(p, q)^{\circ}}(X, H, Y)=K \cap \mathrm{SO}(p, q)^{\circ}$ is a maximal compact subgroup of $\mathcal{Z}_{\mathrm{SO}(p, q)^{\circ}}(X, H, Y)$, where $K$ is the maximal compact subgroup of $\mathcal{Z}_{\mathrm{SO}(p, q)}(X, H, Y)$ as in Lemma 4.19. Let $\widetilde{K}$ be the image of $K_{\mathbb{O}} \times K_{\mathbb{E}}$ under the isomorphism in (4.31). It is evident that $\widetilde{K}$ is a maximal compact subgroup of $\mathcal{Z}_{\mathrm{SO}(p, q)^{\circ}}(X, H, Y)$. Let $\widetilde{M}$ be a maximal compact subgroup of $\mathrm{SO}(p, q)^{\circ}$ containing $\widetilde{K}$. Let $\widetilde{\mathfrak{k}}$ and $\widetilde{\mathfrak{m}}$ be the Lie algebras of $\widetilde{K}$ and $\widetilde{M}$ respectively. As $p \neq 2, q \neq 2$, we have $\widetilde{\mathfrak{m}}=[\widetilde{\mathfrak{m}}, \widetilde{\mathfrak{m}}]$. Then using Theorem 4.2 it follows that, for all $X \neq 0$,

$$
H^{2}\left(\mathcal{O}_{X}, \mathbb{R}\right) \simeq\left[\mathfrak{z}(\widetilde{\mathfrak{k}})^{*}\right]^{\widetilde{K} / \widetilde{K}^{\circ}} .
$$

Let $\mathfrak{k}_{\mathbb{E}}, \mathfrak{k}_{\mathbb{O}}$ be the Lie algebras of $K_{\mathbb{E}}, K_{\mathbb{O}}$ respectively. As $K_{\mathbb{E}}$ is connected, in view of (4.12) we conclude that

$$
\left[\tilde{\mathfrak{z}}(\widetilde{\mathfrak{k}})^{*}\right]^{\widetilde{K} / \widetilde{K}^{\circ}} \simeq \mathfrak{z}\left(\mathfrak{k}_{\mathbb{E}}\right) \oplus\left[\mathfrak{z}\left(\mathfrak{k}_{\mathbb{O}}\right)\right]^{K_{\mathbb{O}} / K_{\mathbb{O}}^{\circ}}
$$

From (4.30) we have $\mathfrak{k}_{\mathbb{E}} \simeq \bigoplus_{\eta \in \mathbb{E}_{\mathbf{d}}} \mathfrak{u}\left(t_{\eta} / 2\right)$. In particular, $\operatorname{dim}_{\mathbb{R}} \mathfrak{z}\left(\mathfrak{k}_{\mathbb{E}}\right)=\# \mathbb{E}_{\mathbf{d}}$. As $\mathbb{N}_{\mathbf{d}_{\mathbb{O}}}=\mathbb{O}_{\mathbf{d}_{\mathbb{Q}}}$,

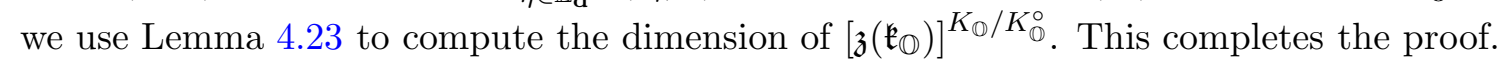

We will next consider the remaining cases which are not covered in Theorem 4.25. These cases are: $(p, q) \in\{(2,1),(1,2)\} ; p>2, q=2$ and $p=2, q>2$. Recall the definition of $\mathcal{Y}_{1}^{\text {even }}(p, q)$ as given in (2.11). If $p>2$, then we list below the set of signed Young diagrams $\mathcal{Y}_{1}^{\text {even }}(p, 2)$ which correspond to non-zero nilpotent orbits in $\mathfrak{s o}(p, 2)$.

a.1 $\left(\left[1^{p-1}, 3^{1}\right],\left(\left(m_{i j}^{1}\right),\left(m_{i j}^{3}\right)\right)\right)$, where $\left(m_{i j}^{1}\right)$ and $\left(m_{i j}^{3}\right)$ are $(p-1) \times 1$ and $1 \times 3$ matrices respectively, satisfying $m_{i 1}^{1}=+1,1 \leq i \leq p-1 ; m_{i 1}^{3}=+1, i=1$ and Yd.2.

a.2 $\left(\left[1^{p-1}, 3^{1}\right],\left(\left(m_{i j}^{1}\right),\left(m_{i j}^{3}\right)\right)\right)$, where $\left(m_{i j}^{1}\right)$ and $\left(m_{i j}^{3}\right)$ are $(p-1) \times 1$ and $1 \times 3$ matrices respectively, satisfying $m_{i 1}^{1}=+1,1 \leq i \leq p-2, m_{i 1}^{1}=-1, i=p-1 ; m_{i 1}^{3}=-1, i=1$ and Yd.2.

a.3 $\left(\left[1^{p-3}, 5^{1}\right],\left(\left(m_{i j}^{1}\right),\left(m_{i j}^{5}\right)\right)\right)$, where $\left(m_{i j}^{1}\right)$ and $\left(m_{i j}^{5}\right)$ are $(p-3) \times 1$ and $1 \times 5$ matrices respectively, satisfying $m_{i 1}^{1}=+1,1 \leq i \leq p-3 ; m_{i 1}^{5}=+1, i=1$ and $\mathbf{Y d} .2$.

a.4 $\left(\left[1^{p-2}, 2^{2}\right],\left(\left(m_{i j}^{1}\right),\left(m_{i j}^{2}\right)\right)\right)$, where $\left(m_{i j}^{1}\right)$ and $\left(m_{i j}^{2}\right)$ are $(p-2) \times 1$ and $2 \times 2$ matrices respectively, satisfying $m_{i 1}^{1}=+1,1 \leq i \leq p-2 ; m_{i 1}^{2}=+1,1 \leq i \leq 2$ and Yd.2.

Similarly as above, if $q>2$, then set $\mathcal{Y}_{1}^{\text {even }}(2, q)$ consists of four elements which correspond to non-zero nilpotent orbits in $\mathfrak{s o}(2, q)$. These are listed below:

b.1 $\left(\left[1^{q-1}, 3^{1}\right],\left(\left(m_{i j}^{1}\right),\left(m_{i j}^{3}\right)\right)\right)$, where $\left(m_{i j}^{1}\right)$ and $\left(m_{i j}^{3}\right)$ are $(q-1) \times 1$ and $1 \times 3$ matrices respectively, satisfying $m_{i 1}^{1}=-1,1 \leq i \leq q-1 ; m_{i 1}^{3}=-1, i=1$ and Yd.2.

b.2 $\left(\left[1^{q-1}, 3^{1}\right],\left(\left(m_{i j}^{1}\right),\left(m_{i j}^{3}\right)\right)\right)$, where $\left(m_{i j}^{1}\right)$ and $\left(m_{i j}^{3}\right)$ are $(q-1) \times 1$ and $1 \times 3$ matrices respectively, satisfying $m_{i 1}^{1}=+1, i=1, m_{i 1}^{1}=-1,2 \leq i \leq q-1 ; m_{i 1}^{3}=+1, i=1$ and Yd.2.

b.3 $\left(\left[1^{q-3}, 5^{1}\right],\left(\left(m_{i j}^{1}\right),\left(m_{i j}^{5}\right)\right)\right)$, where $\left(m_{i j}^{1}\right)$ and $\left(m_{i j}^{5}\right)$ are $(q-3) \times 1$ and $1 \times 5$ matrices respectively, satisfying $m_{i 1}^{1}=-1,1 \leq i \leq q-3 ; m_{i 1}^{5}=-1, i=1$ and Yd.2.

b.4 $\left(\left[1^{q-2}, 2^{2}\right],\left(\left(m_{i j}^{1}\right),\left(m_{i j}^{2}\right)\right)\right)$, where $\left(m_{i j}^{1}\right)$ and $\left(m_{i j}^{2}\right)$ are $(q-2) \times 1$ and $2 \times 2$ matrices respectively, satisfying $m_{i 1}^{1}=-1,1 \leq i \leq q-2 ; m_{i 1}^{2}=+1,1 \leq i \leq 2$ and Yd.2.

Theorem 4.26. Let $\Psi_{\mathrm{SO}(p, q)^{\circ}}: \mathcal{N}\left(\mathrm{SO}(p, q)^{\circ}\right) \longrightarrow \mathcal{Y}_{1}^{\text {even }}(p, q)$ be the parametrization in Theorem 4.16. Let $\mathcal{O}_{X} \in \mathcal{N}\left(\mathrm{SO}(p, q)^{\circ}\right)$. Then the following hold:

(1) Suppose $(p, q) \in\{(2,1),(1,2)\}$, then $H^{2}\left(\mathcal{O}_{X}, \mathbb{R}\right)=0$. 
(2) Assume that $p>2, q=2$.

(i) If $\Psi_{\mathrm{SO}(p, 2)^{\circ}}\left(\mathcal{O}_{X}\right)$ is as in (a.1), then $\operatorname{dim}_{\mathbb{R}} H^{2}\left(\mathcal{O}_{X}, \mathbb{R}\right)=0$.

(ii) If $\Psi_{\mathrm{SO}(p, 2)^{\circ}}\left(\mathcal{O}_{X}\right)$ is as in $(\mathbf{a} .2)$, then $\operatorname{dim}_{\mathbb{R}} H^{2}\left(\mathcal{O}_{X}, \mathbb{R}\right)= \begin{cases}1 & \text { if } p=4 \\ 0 & \text { otherwise. }\end{cases}$

(iii) If $\Psi_{\mathrm{SO}(p, 2)^{\circ}}\left(\mathcal{O}_{X}\right)$ is as in (a.3), then $\operatorname{dim}_{\mathbb{R}} H^{2}\left(\mathcal{O}_{X}, \mathbb{R}\right)=0$.

(iv) If $\Psi_{\mathrm{SO}(p, 2)^{\circ}}\left(\mathcal{O}_{X}\right)$ is as in (a.4), then $\operatorname{dim}_{\mathbb{R}} H^{2}\left(\mathcal{O}_{X}, \mathbb{R}\right)= \begin{cases}1 & \text { if } p=4 \\ 0 & \text { otherwise. }\end{cases}$

(3) Assume $p=2$ and $q>2$.

(i) If $\Psi_{\mathrm{SO}(2, q)^{\circ}}\left(\mathcal{O}_{X}\right)$ is as in (b.1), then $\operatorname{dim}_{\mathbb{R}} H^{2}\left(\mathcal{O}_{X}, \mathbb{R}\right)=0$.

(ii) If $\Psi_{\mathrm{SO}(2, q)^{\circ}}\left(\mathcal{O}_{X}\right)$ is as in $(\mathbf{b} .2)$, then $\operatorname{dim}_{\mathbb{R}} H^{2}\left(\mathcal{O}_{X}, \mathbb{R}\right)= \begin{cases}1 & \text { if } q=4 \\ 0 & \text { otherwise. }\end{cases}$

(iii) If $\Psi_{\mathrm{SO}(2, q)^{\circ}}\left(\mathcal{O}_{X}\right)$ is as in (b.3), then $\operatorname{dim}_{\mathbb{R}} H^{2}\left(\mathcal{O}_{X}, \mathbb{R}\right)=0$.

(iv) If $\Psi_{\mathrm{SO}(2, q)^{\circ}}\left(\mathcal{O}_{X}\right)$ is as in (b.4), then $\operatorname{dim}_{\mathbb{R}} H^{2}\left(\mathcal{O}_{X}, \mathbb{R}\right)= \begin{cases}1 & \text { if } q=4 \\ 0 & \text { otherwise. }\end{cases}$

Proof. As $X \neq 0$, we may assume that $X$ lies in a $\mathfrak{s l}_{2}(\mathbb{R})$-triple, say $\{X, H, Y\}$, in $\mathfrak{s o}(p, q)$.

Proof of (1): Let $\mathfrak{m}$ be the Lie algebra of a maximal compact subgroup of $\mathrm{SO}(p, q)^{\circ}$. As $(p, q) \in$ $\{(2,1),(1,2)\}$, we have $[\mathfrak{m}, \mathfrak{m}]=0$. Thus using Theorem 4.2 it follows that $H^{2}\left(\mathcal{O}_{X}, \mathbb{R}\right)=0$.

Proof of (2): As $\mathbb{N}_{\mathbf{d}}=\mathbb{O}_{\mathbf{d}}$ in each of the cases (i), (ii) and (iii), we will use Proposition 4.22. Let $K$ be the maximal compact subgroup of $\mathcal{Z}_{\mathrm{SO}(p, 2)}(X, H, Y)$ as given in Lemma 4.19. Let $M$ be the maximal compact subgroup of $\mathrm{SO}(p, 2)$ which leaves invariant simultaneously the two subspaces spanned by $\mathcal{H}_{+}$and $\mathcal{H}_{-}$, where $\mathcal{H}_{+}$and $\mathcal{H}_{-}$are as in $(4.28)$ with $q=2$. Then $M^{\circ}=M \cap \mathrm{SO}(p, 2)$ is a maximal compact subgroup of $\mathrm{SO}(p, 2)^{\circ}$. Recall that

$$
K_{\mathbb{O}}:=K \cap M^{\circ}=K \cap \mathrm{SO}(p, 2)^{\circ}
$$

is a maximal compact subgroup of $\mathcal{Z}_{\mathrm{SO}(p, 2)^{\circ}}(X, H, Y)$. Then, in the notation of Proposition $4.22, \Lambda_{\mathcal{H}}\left(K_{\mathbb{O}}\right) \subset \mathrm{SO}(p) \times \mathrm{SO}(2)$. Let $\mathfrak{k}_{\mathbb{O}}$ and $\mathfrak{m}$ be the Lie algebras of $K_{\mathbb{O}}$ and $M^{\circ}$ respectively.

We now prove (i) of (2). Suppose $\Psi_{\mathrm{SO}(p, 2)^{\circ}}\left(\mathcal{O}_{X}\right)$ is as in (a.1). Using Proposition 4.22 it follows that

$$
\begin{aligned}
\Lambda_{\mathcal{H}}\left(K_{\mathbb{O}}\right) & =\left\{\mathbf{D}_{p}(g) \oplus \mathbf{D}_{q}(g) \mid g \in \mathrm{O}_{p-1} \times \mathrm{O}_{1}, \chi_{p}(g)=1, \chi_{q}(g)=1\right\} \\
& =\left\{C \oplus E \bigoplus E \oplus E \mid C \in \mathrm{O}_{p-1}, E \in \mathrm{O}_{1}, \operatorname{det} C \operatorname{det} E=1\right\} .
\end{aligned}
$$

Therefore, $\mathfrak{z}\left(\mathfrak{k}_{\mathbb{O}}\right) \cap[\mathfrak{m}, \mathfrak{m}]=\mathfrak{s o}_{2}$ when $p=3$, and $\mathfrak{z}\left(\mathfrak{k}_{\mathbb{O}}\right)=0$ when $p>3$. From (4.32) it follows that $K_{\mathbb{O}} \simeq S\left(\mathrm{O}_{2} \times \mathrm{O}_{1}\right)$ when $p=3$. Since $\mathrm{O}_{2} / \mathrm{SO}_{2}$ acts non-trivially on $\mathfrak{s o}_{2}$, when $p=3$ we have $\left[\mathfrak{z}\left(\mathfrak{k}_{\mathbb{O}}\right) \cap[\mathfrak{m}, \mathfrak{m}]\right]^{K / K^{\circ}}=0$. Thus using Theorem 4.2,

$$
H^{2}\left(\mathcal{O}_{X}, \mathbb{R}\right)=0
$$

for all $p>2$.

We next give a proof of (ii) of (2). Assume that $\Psi_{\mathrm{SO}(p, 2)^{\circ}}\left(\mathcal{O}_{X}\right)$ is as in (a.2). Using Proposition 4.22 and notation therein,

$$
\begin{aligned}
\Lambda_{\mathcal{H}}\left(K_{\mathbb{O}}\right) & =\left\{\mathbf{D}_{p}(g) \oplus \mathbf{D}_{q}(g) \mid g \in \mathrm{O}_{p-2} \times \mathrm{O}_{1} \times \mathrm{O}_{1}, \chi_{p}(g)=1, \chi_{q}(g)=1\right\} \\
& =\left\{C \oplus F \oplus F \bigoplus D \oplus F \mid C \in \mathrm{O}_{p-2} ; D, F \in \mathrm{O}_{1} ; \operatorname{det} C=1, \operatorname{det} D \operatorname{det} F=1\right\} .
\end{aligned}
$$


It is clear from above that $\mathfrak{z}\left(\mathfrak{k}_{\mathbb{O}}\right) \cap[\mathfrak{m}, \mathfrak{m}]=\mathfrak{s o}_{2}$ when $p=4$ and $\mathfrak{z}\left(\mathfrak{k}_{\mathbb{O}}\right)=0$ when $p \neq 4, p>2$. When $p=4$, then $K_{\mathbb{O}} \simeq \mathrm{SO}_{2} \times S\left(\mathrm{O}_{1} \times \mathrm{O}_{1}\right)$ from (4.33). As $\mathrm{SO}_{2}$ acts trivially on $\mathfrak{s o}_{2}$, using Theorem 4.2 we conclude that

$$
\operatorname{dim}_{\mathbb{R}} H^{2}\left(\mathcal{O}_{X}, \mathbb{R}\right)= \begin{cases}1 & \text { if } p=4 \\ 0 & \text { otherwise }\end{cases}
$$

We now give a proof of (iii) of (2). Assume that $\Psi_{\mathrm{SO}(p, 2)^{\circ}}\left(\mathcal{O}_{X}\right)$ is as in (a.3). Using Proposition 4.22 and notation therein,

$$
\begin{aligned}
\Lambda_{\mathcal{H}}\left(K_{\mathbb{O}}\right) & =\left\{\mathbf{D}_{p}(g) \oplus \mathbf{D}_{q}(g) \mid g \in \mathrm{O}_{p-3} \times \mathrm{O}_{1}, \chi_{p}(g)=1, \chi_{q}(g)=1\right\} \\
& =\left\{C \oplus E \oplus E \oplus E \bigoplus E \oplus E \mid C \in \mathrm{O}_{p-3}, E \in \mathrm{O}_{1}, \operatorname{det} C \operatorname{det} E=1\right\} .
\end{aligned}
$$

Therefore, we have $\mathfrak{z}\left(\mathfrak{k}_{\mathbb{O}}\right) \cap[\mathfrak{m}, \mathfrak{m}]=\mathfrak{s o}_{2}$ when $p=5$, and $\mathfrak{z}\left(\mathfrak{k}_{\mathbb{O}}\right)=0$ for $p>2, p \neq 5$. It follows from (4.34) that $K_{\mathbb{O}} \simeq S\left(\mathrm{O}_{2} \times \mathrm{O}_{1}\right)$ when $p=5$. Since $\mathrm{O}_{2} / \mathrm{SO}_{2}$ acts non-trivially on $\mathfrak{s o}_{2}$, in the case when $p=5$ we have $\left[\mathfrak{z}\left(\mathfrak{k}_{\mathbb{O}}\right) \cap[\mathfrak{m}, \mathfrak{m}]\right]^{K / K^{\circ}}=0$. Thus in view of Theorem 4.2,

$$
H^{2}\left(\mathcal{O}_{X}, \mathbb{R}\right)=0
$$

for all $p>2$.

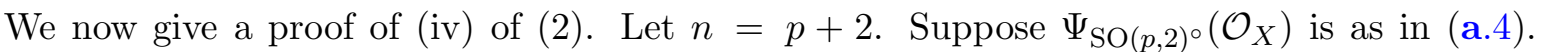
We need to construct a standard orthogonal basis as done before. We follow the notation as in Lemma 4.20. Define, $\mathcal{A}_{+}(2):=\left(\left(v_{1}^{2}+X v_{2}^{2}\right) / \sqrt{2},\left(v_{2}^{2}-X v_{1}^{2}\right) / \sqrt{2}\right)$ and

$$
\mathcal{A}_{-}(2):=\left(\left(v_{1}^{2}-X v_{2}^{2}\right) / \sqrt{2},\left(v_{2}^{2}+X v_{1}^{2}\right) / \sqrt{2}\right) .
$$

Finally set $\mathcal{H}_{+}:=\mathcal{B}^{0}(1) \vee \mathcal{A}_{+}(2), \mathcal{H}_{-}:=\mathcal{A}_{-}(2)$ and $\mathcal{H}:=\mathcal{H}_{+} \vee \mathcal{H}_{-}$. Then it is clear that $\mathcal{H}$ is a standard orthogonal basis of $V$ such that

$$
\mathcal{H}_{+}=\{v \in \mathcal{H} \mid\langle v, v\rangle=1\}
$$

and $\mathcal{H}_{-}=\{v \in \mathcal{H} \mid\langle v, v\rangle=-1\}$. In particular, $\# \mathcal{H}_{+}=p$ and $\# \mathcal{H}_{-}=2$. Let $V_{+}(2)$, $V_{-}(2)$ be the spans of $\mathcal{A}_{+}(2), \mathcal{A}_{-}(2)$ respectively. Let $K$ be the maximal compact subgroup of $\mathcal{Z}_{\mathrm{SO}(p, 2)}(X, H, Y)$ as in Lemma 4.20. We observe that if $g \in K$, then $g\left(V_{+}(2)\right) \subset V_{+}(2)$, $g\left(V_{-}(2)\right) \subset V_{-}(2)$ and

$$
\left[\left.g\right|_{V_{+}(2)}\right]_{\mathcal{A}_{+}(2)}=\left[\left.g\right|_{V_{-}(2)}\right]_{\mathcal{A}_{-}(2)}=\left[\left.g\right|_{L(1)}\right]_{\mathcal{B}^{0}(2)} .
$$

Let $\Lambda_{\mathcal{H}}: \operatorname{End}_{\mathbb{R}} \mathbb{R}^{n} \longrightarrow \mathrm{M}_{n}(\mathbb{R})$ be the isomorphism of $\mathbb{R}$-algebras induced by the above ordered basis $\mathcal{H}$. Let $M$ be the maximal compact subgroup in $\operatorname{SO}(p, 2)$ which simultaneously leaves the subspaces spanned by $\mathcal{H}_{+}$and $\mathcal{H}_{-}$invariant. Then $M^{\circ}=M \cap \mathrm{SO}(p, 2)^{\circ}$ is a maximal compact subgroup of $\mathrm{SO}(p, 2)^{\circ}$, and $\widetilde{K}:=K \cap M^{\circ}$ is a maximal compact subgroup of $\mathcal{Z}_{\mathrm{SO}(p, 2)^{\circ}}(X, H, Y)$. We have the following explicit description of $\Lambda_{\mathcal{H}}(\widetilde{K}) \subset \mathrm{SO}(p) \times \mathrm{SO}(2)$ :

$$
\Lambda_{\mathcal{H}}(\widetilde{K})=\left\{A \oplus B \bigoplus B \mid A \in \mathrm{O}_{p-2}, B \in \mathrm{O}_{2} ; \operatorname{det} A \operatorname{det} B=1 \text { and } \operatorname{det} B=1\right\} .
$$

In particular, $\widetilde{K} \simeq \mathrm{SO}_{p-2} \times \mathrm{SO}_{2}$. Let $\widetilde{\mathfrak{k}}$ and $\mathfrak{m}$ be the Lie algebras of $\widetilde{K}$ and $M^{\circ}$ respectively. From (4.35),

$$
\mathfrak{z}(\widetilde{\mathfrak{k}}) \cap[\mathfrak{m}, \mathfrak{m}]= \begin{cases}\mathfrak{s o}_{2} & \text { if } p=4 \\ 0 & \text { otherwise. }\end{cases}
$$

As $\widetilde{K}$ is connected, the conclusion follows from Theorem 4.2. This completes the proof of (2).

The proofs of (3)(i), (3)(ii), (3)(iii) and (3)(iv) are similar to those of (2)(i), (2)(ii), (2)(iii) and $(2)$ (iv) respectively and hence the details are omitted. 
4.6. Second cohomology groups of nilpotent orbits in $\mathfrak{s o}^{*}(2 n)$. Let $n$ be a positive integer. The aim in this subsection is to compute the second cohomology groups of nilpotent orbits in the simple real Lie algebra $\mathfrak{s o}^{*}(2 n)$ under the adjoint action of $\mathrm{SO}^{*}(2 n)$. Throughout this subsection $\langle\cdot, \cdot\rangle$ denotes the skew-Hermitian form on $\mathbb{H}^{n}$ defined by $\langle x, y\rangle:=\bar{x}^{t} \mathbf{j} \mathrm{I}_{n} y$, for $x, y \in \mathbb{H}^{n}$. We will follow notation as defined in $\S 2$.

We first need to describe a suitable parametrization of $\mathcal{N}\left(\mathrm{SO}^{*}(2 n)\right)$, the set of all nilpotent orbits in $\mathfrak{s o}^{*}(2 n)$. Let $\Psi_{\mathrm{SL}_{n}(\mathbb{H})}: \mathcal{N}\left(\mathrm{SL}_{n}(\mathbb{H})\right) \longrightarrow \mathcal{P}(n)$ be the parametrization as in Theorem 4.8. As $\mathrm{SO}^{*}(2 n) \subset \mathrm{SL}_{n}(\mathbb{H})$ (consequently, $\left.\mathcal{N}_{\mathfrak{s o}^{*}(2 n)} \subset \mathcal{N}_{\mathfrak{s l}_{n}(\mathbb{H})}\right)$ we have the inclusion map $\Theta_{\mathrm{SO}^{*}(2 n)}: \mathcal{N}\left(\mathrm{SO}^{*}(2 n)\right) \longrightarrow \mathcal{N}\left(\mathrm{SL}_{n}(\mathbb{H})\right)$. Let

$$
\Psi_{\mathrm{SO}^{*}(2 n)}^{\prime}:=\Psi_{\mathrm{SL}_{n}(\mathbb{H})} \circ \Theta_{\mathrm{SO}^{*}(2 n)}: \mathcal{N}\left(\mathrm{SO}^{*}(2 n)\right) \longrightarrow \mathcal{P}(n)
$$

be the composition. Let $X \in \mathfrak{s o}^{*}(2 n)$ be a non-zero nilpotent element and $\mathcal{O}_{X}$ be the corresponding nilpotent orbit in $\mathfrak{s o}^{*}(2 n)$. Let $\{X, H, Y\} \subset \mathfrak{s o}^{*}(2 n)$ be a $\mathfrak{s l}_{2}(\mathbb{R})$-triple. We now apply Proposition 5.14, Remark 5.16(3), and follow the notation used therein. Let $V:=\mathbb{H}^{n}$ be the right $\mathbb{H}$-vector space of column vectors. Let $\left\{d_{1}, \cdots, d_{s}\right\}$, with $d_{1}<\cdots<d_{s}$, be the ordered finite set of natural numbers that arise as $\mathbb{R}$-dimension of non-zero irreducible $\operatorname{Span}_{\mathbb{R}}\{X, H, Y\}$ submodules of $V$. Recall that $M(d-1)$ is defined to be the isotypical component of $V$ containing all irreducible $\operatorname{Span}_{\mathbb{R}}\{X, H, Y\}$-submodules of $V$ with highest weight $(d-1)$, and as in (2.2), we set $L(d-1):=V_{Y, 0} \cap M(d-1)$. Recall that the space $L\left(d_{r}-1\right)$ is a $\mathbb{H}$-subspace for $1 \leq r \leq s$. Let $t_{d_{r}}:=\operatorname{dim}_{\mathbb{H}} L\left(d_{r}-1\right), 1 \leq r \leq s$. Then $\mathbf{d}:=\left[d_{1}^{t_{d_{1}}}, \cdots, d_{s}^{t_{d_{s}}}\right] \in \mathcal{P}(n)$, and moreover, $\Psi_{\mathrm{SO}^{*}(2 n)}^{\prime}\left(\mathcal{O}_{X}\right)=\mathbf{d}$.

We next assign $\operatorname{sgn}_{\mathcal{O}_{X}} \in \mathcal{S}_{\mathbf{d}}^{\text {odd }}(n)$ to each $\mathcal{O}_{X} \in \mathcal{N}\left(\mathrm{SO}^{*}(2 n)\right)$; see (2.9) for the definition of $\mathcal{S}_{\mathbf{d}}^{\text {odd }}(n)$. For each $d \in \mathbb{N}_{\mathbf{d}}$ (see $(2.3)$ for the definition of $\mathbb{N}_{\mathbf{d}}$ ) we will define a $t_{d} \times d$ matrix $\left(m_{i j}^{d}\right)$ in $\mathbf{A}_{d}$ that depends only on the orbit $\mathcal{O}_{X}$; see (2.5) for the definition of $\mathbf{A}_{d}$. For this, recall that the form $(\cdot, \cdot)_{d}: L(d-1) \times L(d-1) \longrightarrow \mathbb{H}$ defined as in (4.6) is skew-Hermitian or Hermitian according as $d$ is odd or even. Denoting the signature of $(\cdot, \cdot)_{\eta}$ by $\left(p_{\eta}, q_{\eta}\right)$ when $\eta \in \mathbb{E}_{\mathbf{d}}$, we now define

$$
\begin{aligned}
& m_{i 1}^{\theta}:=+1 \quad \text { if } 1 \leq i \leq t_{\theta}, \quad \theta \in \mathbb{O}_{\mathbf{d}} \\
& m_{i 1}^{\eta}:=\left\{\begin{array}{ll}
+1 & \text { if } 1 \leq i \leq p_{\eta} \\
-1 & \text { if } p_{\eta}<i \leq t_{\eta}
\end{array}, \eta \in \mathbb{E}_{\mathbf{d}}\right.
\end{aligned}
$$

and for $j>1$ we define $\left(m_{i j}^{d}\right)$ as in (4.15) and (4.16). Then the matrices $\left(m_{i j}^{d}\right)$ clearly verify $(\mathbf{Y d} .2)$. Set $\mathbf{s g n}_{\mathcal{O}_{X}}:=\left(\left(m_{i j}^{d_{1}}\right), \cdots,\left(m_{i j}^{d_{s}}\right)\right)$. It now follows from the above definitions of $m_{i 1}^{\theta}, \theta \in \mathbb{O}_{\mathbf{d}}$ that $\mathbf{s g n}_{\mathcal{O}_{X}} \in \mathcal{S}_{\mathbf{d}}^{\text {odd }}(n)$. Thus we have the map

$$
\Psi_{\mathrm{SO}^{*}(2 n)}: \mathcal{N}\left(\mathrm{SO}^{*}(2 n)\right) \longrightarrow \mathcal{Y}^{\text {odd }}(n), \mathcal{O}_{X} \longmapsto\left(\Psi_{\mathrm{SO}^{*}(2 n)}^{\prime}\left(\mathcal{O}_{X}\right), \operatorname{sgn}_{\mathcal{O}_{X}}\right) ;
$$

where $\mathcal{Y}^{\text {odd }}(n)$ is as in (2.12). The following theorem is standard.

Theorem 4.27 ([CoMc, Theorem 9.3.4]). The above map $\Psi_{\mathrm{SO}^{*}(2 n)}$ is a bijection.

Let $0 \neq X \in \mathcal{N}_{\mathfrak{s o}^{*}(2 n)}$ and $\{X, H, Y\}$ be a $\mathfrak{s l}_{2}(\mathbb{R})$-triple in $\mathfrak{s o}^{*}(2 n)$. Let $\Psi_{\mathrm{SO}^{*}(2 n)}\left(\mathcal{O}_{X}\right)=$ $\left(\mathbf{d}, \operatorname{sgn}_{\mathcal{O}_{X}}\right)$. Then $\Psi_{\mathrm{SO}^{*}(2 n)}^{\prime}\left(\mathcal{O}_{X}\right)=\mathbf{d}$. Recall that $\operatorname{sgn}_{\mathcal{O}_{X}}$ determines the signature of $(\cdot, \cdot)_{\eta}$ on $L(\eta-1)$ for all $\eta \in \mathbb{E}_{\mathbf{d}}$; let $\left(p_{\eta}, q_{\eta}\right)$ be the signature of $(\cdot, \cdot)_{\eta}$ on $L(\eta-1)$. Let $\left(v_{1}^{d}, \cdots, v_{t_{d}}^{d}\right)$ be an ordered $\mathbb{H}$-basis of $L(d-1)$ as in Proposition 5.14. It now follows from Proposition 5.14(3)(a) that $\left(v_{1}^{d}, \cdots, v_{t_{d}}^{d}\right)$ is an orthogonal basis of $L(d-1)$ for the form $(\cdot, \cdot)_{d}$ for all $d \in \mathbb{N}_{\mathbf{d}}$. We also assume that the vectors in the ordered basis $\left(v_{1}^{d}, \cdots, v_{t_{d}}^{d}\right)$ satisfy the properties in Remark $5.16(3)$. Since $(\cdot, \cdot)_{\theta}$ is skew-Hermitian for all $\theta \in \mathbb{O}_{\mathbf{d}}$, using Lemma 2.3, we may assume that 
$\left(v_{1}^{\theta}, \cdots, v_{t_{\theta}}^{\theta}\right)$ is a standard orthogonal basis for all $\theta \in \mathbb{O}_{\mathbf{d}}$. Thus

$$
\left(v_{j}^{\theta}, v_{j}^{\theta}\right)_{\theta}=\mathbf{j} \quad \text { for all } 1 \leq j \leq t_{\theta}, \theta \in \mathbb{O}_{\mathbf{d}} .
$$

In view of the signature of $(\cdot, \cdot)_{\eta}, \eta \in \mathbb{E}_{\mathbf{d}}$, we may assume that

$$
\left(v_{j}^{\eta}, v_{j}^{\eta}\right)_{\eta}= \begin{cases}+1 & \text { if } 1 \leq j \leq p_{\eta} \\ -1 & \text { if } p_{\eta}<j \leq t_{\eta} .\end{cases}
$$

For $\eta \in \mathbb{E}_{\mathbf{d}}, 1 \leq r \leq p_{\eta}$, define

$$
w_{r l}^{\eta}:= \begin{cases}\left(X^{l} v_{r}^{\eta}+X^{\eta-1-l} v_{r}^{\eta} \mathbf{j}\right) / \sqrt{2} & \text { if } l \text { is even, } 0 \leq l \leq \eta / 2-1 \\ \left(X^{l} v_{r}^{\eta}+X^{\eta-1-l} v_{r}^{\eta} \mathbf{j}\right) \mathbf{i} / \sqrt{2} & \text { if } l \text { is odd, } 0 \leq l \leq \eta / 2-1 \\ \left(X^{\eta-1-l} v_{r}^{\eta}-X^{l} v_{r}^{\eta} \mathbf{j}\right) \mathbf{i} / \sqrt{2} & \text { if } l \text { is odd, } \eta / 2 \leq l \leq \eta-1 \\ \left(X^{\eta-1-l} v_{r}^{\eta}-X^{l} v_{r}^{\eta} \mathbf{j}\right) / \sqrt{2} & \text { if } l \text { is even, } \eta / 2 \leq l \leq \eta-1 .\end{cases}
$$

Similarly for $\eta \in \mathbb{E}_{\mathbf{d}}, p_{\eta}<r \leq t_{\eta}$, define

$$
w_{r l}^{\eta}:= \begin{cases}\left(X^{l} v_{r}^{\eta}+X^{\eta-1-l} v_{r}^{\eta} \mathbf{j}\right) \mathbf{i} / \sqrt{2} & \text { if } l \text { is even, } 0 \leq l \leq \eta / 2-1 \\ \left(X^{l} v_{r}^{\eta}+X^{\eta-1-l} v_{r}^{\eta} \mathbf{j}\right) / \sqrt{2} & \text { if } l \text { is odd, } 0 \leq l \leq \eta / 2-1 \\ \left(X^{\eta-1-l} v_{r}^{\eta}-X^{l} v_{r}^{\eta} \mathbf{j}\right) / \sqrt{2} & \text { if } l \text { is odd, } \eta / 2 \leq l \leq \eta-1 \\ \left(X^{\eta-1-l} v_{r}^{\eta}-X^{l} v_{r}^{\eta} \mathbf{j}\right) \mathbf{i} / \sqrt{2} & \text { if } l \text { is even, } \eta / 2 \leq l \leq \eta-1 .\end{cases}
$$

Using (4.37) we observe that for all $\eta \in \mathbb{E}_{\mathbf{d}}$,

$$
\left\{w_{r l}^{\eta} \mid 0 \leq l \leq \eta-1,1 \leq r \leq t_{\eta}\right\}
$$

is an orthogonal basis of $M(\eta-1)$ with respect to $\langle\cdot, \cdot\rangle$, where $\left\langle w_{r l}^{\eta}, w_{r l}^{\eta}\right\rangle=\mathbf{j}$ for $0 \leq l \leq \eta-1$, $1 \leq r \leq t_{\eta}$. For $\eta \in \mathbb{E}_{\mathbf{d}}, 0 \leq l \leq \eta / 2-1$, set

$$
W^{l}(\eta):=\operatorname{Span}_{\mathbb{H}}\left\{w_{r l}^{\eta}, w_{r \eta-1-l}^{\eta} \mid 1 \leq r \leq t_{\eta}\right\} .
$$

Moreover, we define a standard orthogonal basis $\mathcal{D}^{l}(\eta)$ of $W^{l}(\eta)$ with respect to $\langle\cdot, \cdot\rangle$ as follows:

$$
\mathcal{D}^{l}(\eta):= \begin{cases}\left(w_{1 l}^{\eta}, \cdots, w_{p_{\eta} l}^{\eta}\right) \vee\left(w_{1(\eta-1-l)}^{\eta}, \cdots, w_{p_{\eta}(\eta-1-l)}^{\eta}\right) \\ \bigvee\left(w_{\left(p_{\eta}+1\right)(\eta-1-l)}^{\eta}, \cdots, w_{t_{\eta}(\eta-1-l)}^{\eta}\right) \vee\left(w_{\left(p_{\eta}+1\right) l}^{\eta}, \cdots, w_{t_{\eta} l}^{\eta}\right) & \text { if } l \text { is even } \\ \left(w_{1(\eta-1-l)}^{\eta}, \cdots, w_{p_{\eta}(\eta-1-l)}^{\eta}\right) \vee\left(w_{1 l}^{\eta}, \cdots, w_{p_{\eta} l}^{\eta}\right) & \\ \vee\left(w_{\left(p_{\eta}+1\right) l}^{\eta}, \cdots, w_{t_{\eta} l}^{\eta}\right) \vee\left(w_{\left(p_{\eta}+1\right)(\eta-1-l)}^{\eta}, \cdots, w_{t_{\eta}(\eta-1-l)}^{\eta}\right) & \text { if } l \text { is odd } .\end{cases}
$$

Now fixing $\theta \in \mathbb{O}_{\mathbf{d}}^{1}$, for all $1 \leq r \leq t_{\theta}$, define

$$
w_{r l}^{\theta}:= \begin{cases}\left(X^{l} v_{r}^{\theta}+X^{\theta-1-l} v_{r}^{\theta}\right) / \sqrt{2} & \text { if } l \text { is even, } 0 \leq l<(\theta-1) / 2 \\ \left(X^{l} v_{r}^{\theta}+X^{\theta-1-l} v_{r}^{\theta}\right) \mathbf{i} / \sqrt{2} & \text { if } l \text { is odd, } 0 \leq l<(\theta-1) / 2 \\ X^{l} v_{r}^{\theta} & \text { if } l=(\theta-1) / 2 \\ \left(X^{\theta-1-l} v_{r}^{\theta}-X^{l} v_{r}^{\theta}\right) / \sqrt{2} & \text { if } l \text { is odd, }(\theta+1) / 2 \leq l \leq \theta-1 \\ \left(X^{\theta-1-l} v_{r}^{\theta}-X^{l} v_{r}^{\theta}\right) \mathbf{i} / \sqrt{2} & \text { if } l \text { is even, }(\theta+1) / 2 \leq l \leq \theta-1 .\end{cases}
$$


For all $\zeta \in \mathbb{O}_{\mathbf{d}}^{3}$ and $1 \leq r \leq t_{\zeta}$, define

$$
w_{r l}^{\zeta}:= \begin{cases}\left(X^{l} v_{r}^{\zeta}+X^{\zeta-1-l} v_{r}^{\zeta}\right) / \sqrt{2} & \text { if } l \text { is even, } 0 \leq l<(\zeta-1) / 2 \\ \left(X^{l} v_{r}^{\zeta}+X^{\zeta-1-l} v_{r}^{\zeta}\right) \mathbf{i} / \sqrt{2} & \text { if } l \text { is odd, } 0 \leq l<(\zeta-1) / 2 \\ X^{l} v_{r}^{\zeta} \mathbf{i} & \text { if } l=(\zeta-1) / 2 \\ \left(X^{\zeta-1-l} v_{r}^{\zeta}-X^{l} v_{r}^{\zeta}\right) / \sqrt{2} & \text { if } l \text { is odd, }(\zeta+1) / 2 \leq l \leq \zeta-1 \\ \left(X^{\zeta-1-l} v_{r}^{\zeta}-X^{l} v_{r}^{\zeta}\right) \mathbf{i} / \sqrt{2} & \text { if } l \text { is even, }(\zeta+1) / 2 \leq l \leq \zeta-1 .\end{cases}
$$

Using (4.36) we observe that for all $\theta \in \mathbb{O}_{\mathbf{d}}$,

$$
\left\{w_{r l}^{\theta} \mid 0 \leq l \leq \theta-1,1 \leq r \leq t_{\theta}\right\}
$$

is an orthogonal basis of $M(\theta-1)$ with respect to $\langle\cdot, \cdot\rangle$, where $\left\langle w_{r l}^{\theta}, w_{r l}^{\theta}\right\rangle=\mathbf{j}$ for $0 \leq l \leq \theta-1$, $1 \leq r \leq t_{\theta}$. For each $\theta \in \mathbb{O}_{\mathbf{d}}, 0 \leq l \leq \theta-1$, set

$$
V^{l}(\theta):=\operatorname{Span}_{\mathbb{H}}\left\{w_{r l}^{\theta} \mid 1 \leq r \leq t_{\theta}\right\} .
$$

The standard orthogonal ordered basis $\left(w_{1 l}^{\theta}, \cdots, w_{t_{\theta} l}^{\theta}\right)$ of $V^{l}(\theta)$ with respect to $\langle\cdot, \cdot\rangle$ is denoted by $\mathcal{C}^{l}(\theta)$.

Let $W$ be a right $\mathbb{H}$-vector space and $\langle\cdot, \cdot\rangle^{\prime}$ a non-degenerate skew-Hermitian form on $W$. Let $\operatorname{dim}_{\mathbb{H}} W=m$, and let $\mathcal{B}^{\prime}:=\left(v_{1}, \cdots, v_{m}\right)$ be a standard orthogonal basis of $W$ such that $\left\langle v_{r}, v_{r}\right\rangle^{\prime}=\mathbf{j}$ for all $1 \leq r \leq m$. Define

$$
\mathrm{J}_{\mathcal{B}^{\prime}}: W \longrightarrow W, \sum_{r} v_{r} z_{r} \longmapsto \sum_{r} v_{r} \mathbf{j} z_{r}
$$

for all column vectors $\left(z_{1}, \cdots, z_{m}\right)^{t} \in \mathbb{H}^{m}$. Set

$$
K_{\mathcal{B}^{\prime}}:=\left\{g \in \mathrm{SO}^{*}\left(W,\langle\cdot, \cdot\rangle^{\prime}\right) \mid g \mathrm{~J}_{\mathcal{B}^{\prime}}=\mathrm{J}_{\mathcal{B}^{\prime}} g\right\} .
$$

The following lemma is standard; its proof is omitted.

Lemma 4.28. Let $W,\langle\cdot, \cdot\rangle^{\prime}$ and $\mathcal{B}^{\prime}$ be as above. Then the following hold:

(1) $K_{\mathcal{B}^{\prime}}$ is a maximal compact subgroup of $\mathrm{SO}^{*}\left(W,\langle\cdot, \cdot\rangle^{\prime}\right)$.

(2) $K_{\mathcal{B}^{\prime}}=\left\{g \in \mathrm{SL}(W) \mid[g]_{\mathcal{B}^{\prime}}=A+\mathbf{j} B\right.$ with $\left.A, B \in \mathrm{M}_{m}(\mathbb{R}), A+\sqrt{-1} B \in \mathrm{U}(m)\right\}$.

Recall that $\left\{x \in \operatorname{End}_{\mathbb{H}} W \mid x \mathrm{~J}_{\mathcal{B}^{\prime}}=\mathrm{J}_{\mathcal{B}^{\prime}} x\right\}=\left\{x \in \operatorname{End}_{\mathbb{H}} W \mid[x]_{\mathcal{B}^{\prime}} \in \mathrm{M}_{m}(\mathbb{R})+\mathbf{j} \mathrm{M}_{m}(\mathbb{R})\right\}$. We now consider the $\mathbb{R}$-algebra isomorphism

$$
\Lambda_{\mathcal{B}^{\prime}}^{\prime}:\left\{x \in \operatorname{End}_{\mathbb{H}} W \mid x \mathrm{~J}_{\mathcal{B}^{\prime}}=\mathrm{J}_{\mathcal{B}^{\prime}} x\right\} \longrightarrow \mathrm{M}_{m}(\mathbb{C}), x \longmapsto A+\sqrt{-1} B,
$$

where $A, B \in \mathrm{M}_{m}(\mathbb{R})$ are the unique elements such that $[x]_{\mathcal{B}}=A+\mathbf{j} B$. In view of the above lemma it is clear that $\Lambda_{\mathcal{B}^{\prime}}^{\prime}\left(K_{\mathcal{B}^{\prime}}\right)=\mathrm{U}(m)$, and hence $\Lambda_{\mathcal{B}^{\prime}}^{\prime}: K_{\mathcal{B}^{\prime}} \longrightarrow \mathrm{U}(m)$ is an isomorphism of Lie groups.

In the next lemma we specify a maximal compact subgroup of $\mathcal{Z}_{\mathrm{SO}^{*}(2 n)}(X, H, Y)$ which will be used in Proposition 4.30. Recall that $\bar{Z}:=\left(\sigma_{c}\left(z_{r l}\right)\right) \in \mathrm{M}_{m}(\mathbb{H})$; see Section 2.1.

Lemma 4.29. Let $K$ be the subgroup of $\mathcal{Z}_{\mathrm{SO}^{*}(2 n)}(X, H, Y)$ consisting of elements $g$ in $\mathcal{Z}_{\mathrm{SO}^{*}(2 n)}(X, H, Y)$ satisfying the following conditions:

(1) $g\left(V^{l}(\theta)\right) \subset V^{l}(\theta)$ for all $\theta \in \mathbb{O}_{\mathbf{d}}$ and $0 \leq l \leq \theta-1$. 
(2) For all $\theta \in \mathbb{O}_{\mathbf{d}}^{1}$, there exist $A_{\theta}, B_{\theta} \in \mathrm{M}_{t_{\theta}}(\mathbb{R})$ with $A_{\theta}+\sqrt{-1} B_{\theta} \in \mathrm{U}\left(t_{\theta}\right)$ such that

$$
\left[\left.g\right|_{V^{l}(\theta)}\right]_{\mathcal{C}^{l}(\theta)}= \begin{cases}A_{\theta}+\mathbf{j} B_{\theta} & \text { if } l \text { is even, } 0 \leq l<(\theta-1) / 2 \\ A_{\theta}-\mathbf{j} B_{\theta} & \text { if } l \text { is odd, } 0 \leq l<(\theta-1) / 2 \\ A_{\theta}+\mathbf{j} B_{\theta} & \text { if } l=(\theta-1) / 2 \\ A_{\theta}+\mathbf{j} B_{\theta} & \text { if } l \text { is odd, }(\theta+1) / 2 \leq l \leq \theta-1 \\ A_{\theta}-\mathbf{j} B_{\theta} & \text { if } l \text { is even, }(\theta+1) / 2 \leq l \leq \theta-1 .\end{cases}
$$

(3) For all $\zeta \in \mathbb{O}_{\mathbf{d}}^{3}$, there exist $A_{\zeta}, B_{\zeta} \in \mathrm{M}_{t_{\zeta}}(\mathbb{R})$ with $A_{\zeta}+\sqrt{-1} B_{\zeta} \in \mathrm{U}\left(t_{\zeta}\right)$ such that

$$
\left[\left.g\right|_{V^{l}(\zeta)}\right]_{\mathcal{C}^{l}(\zeta)}= \begin{cases}A_{\zeta}+\mathbf{j} B_{\zeta} & \text { if } l \text { is even, } 0 \leq l<(\zeta-1) / 2 \\ A_{\zeta}-\mathbf{j} B_{\zeta} & \text { if } l \text { is odd, } 0 \leq l<(\zeta-1) / 2 \\ A_{\zeta}-\mathbf{j} B_{\zeta} & \text { if } l=(\zeta-1) / 2 \\ A_{\zeta}+\mathbf{j} B_{\zeta} & \text { if } l \text { is odd, }(\zeta+1) / 2 \leq l \leq \zeta-1 \\ A_{\zeta}-\mathbf{j} B_{\zeta} & \text { if } l \text { is even, }(\zeta+1) / 2 \leq l \leq \zeta-1 .\end{cases}
$$

(4) $g\left(W^{l}(\eta)\right) \subset W^{l}(\eta)$ for all $\eta \in \mathbb{E}_{\mathbf{d}}$ and $0 \leq l \leq \eta / 2-1$.

(5) For all $\eta \in \mathbb{E}_{\mathbf{d}}$, there exist $A_{p_{\eta}}, B_{p_{\eta}}, C_{p_{\eta}}, D_{p_{\eta}} \in \mathrm{M}_{p_{\eta}}(\mathbb{R}), A_{q_{\eta}}^{\prime}, B_{q_{\eta}}^{\prime}, C_{q_{\eta}}^{\prime}, D_{q_{\eta}}^{\prime} \in \mathrm{M}_{q_{\eta}}(\mathbb{R})$ with $A_{p_{\eta}}+\mathbf{j} B_{p_{\eta}}+\mathbf{i}\left(C_{p_{\eta}}+\mathbf{j} D_{p_{\eta}}\right) \in \operatorname{Sp}\left(p_{\eta}\right)$ and $A_{q_{\eta}}^{\prime}+\mathbf{j} B_{q_{\eta}}^{\prime}+\mathbf{i}\left(C_{q_{\eta}}^{\prime}+\mathbf{j} D_{q_{\eta}}^{\prime}\right) \in \operatorname{Sp}\left(q_{\eta}\right)$ such that

$$
\left[\left.g\right|_{W^{l}(\eta)}\right]_{\mathcal{D}^{l}(\eta)}=\left(\begin{array}{cccc}
A_{p_{\eta}}+\mathbf{j} B_{p_{\eta}} & -C_{p_{\eta}}+\mathbf{j} D_{p_{\eta}} & \\
C_{p_{\eta}}+\mathbf{j} D_{p_{\eta}} & A_{p_{\eta}}-\mathbf{j} B_{p_{\eta}} & & \\
& & A_{q_{\eta}}^{\prime}+\mathbf{j} B_{q_{\eta}}^{\prime} & -C_{q_{\eta}}^{\prime}+\mathbf{j} D_{q_{\eta}}^{\prime} \\
& & C_{q_{\eta}}^{\prime}+\mathbf{j} D_{q_{\eta}}^{\prime} & A_{q_{\eta}}^{\prime}-\mathbf{j} B_{q_{\eta}}^{\prime}
\end{array}\right)
$$

Then $K$ is a maximal compact subgroup of $\mathcal{Z}_{\mathrm{SO}^{*}(2 n)}(X, H, Y)$.

Proof. Let $\mathcal{B}^{l}(d)=\left(X^{l} v_{1}^{d}, \cdots, X^{l} v_{t_{d}}^{d}\right)$ be the ordered basis of $X^{l} L(d-1)$ for $0 \leq l \leq d-1$, $d \in \mathbb{N}_{\mathbf{d}}$, as in (4.3). Let $K^{\prime}$ be the subgroup consisting of all $g \in \mathcal{Z}_{\mathrm{SO}^{*}(2 n)}(X, H, Y)$ satisfying 
the following properties:

For $\theta \in \mathbb{O}_{\mathbf{d}}, 0 \leq l \leq \theta-1, g\left(V^{l}(\theta)\right) \subset V^{l}(\theta)$,

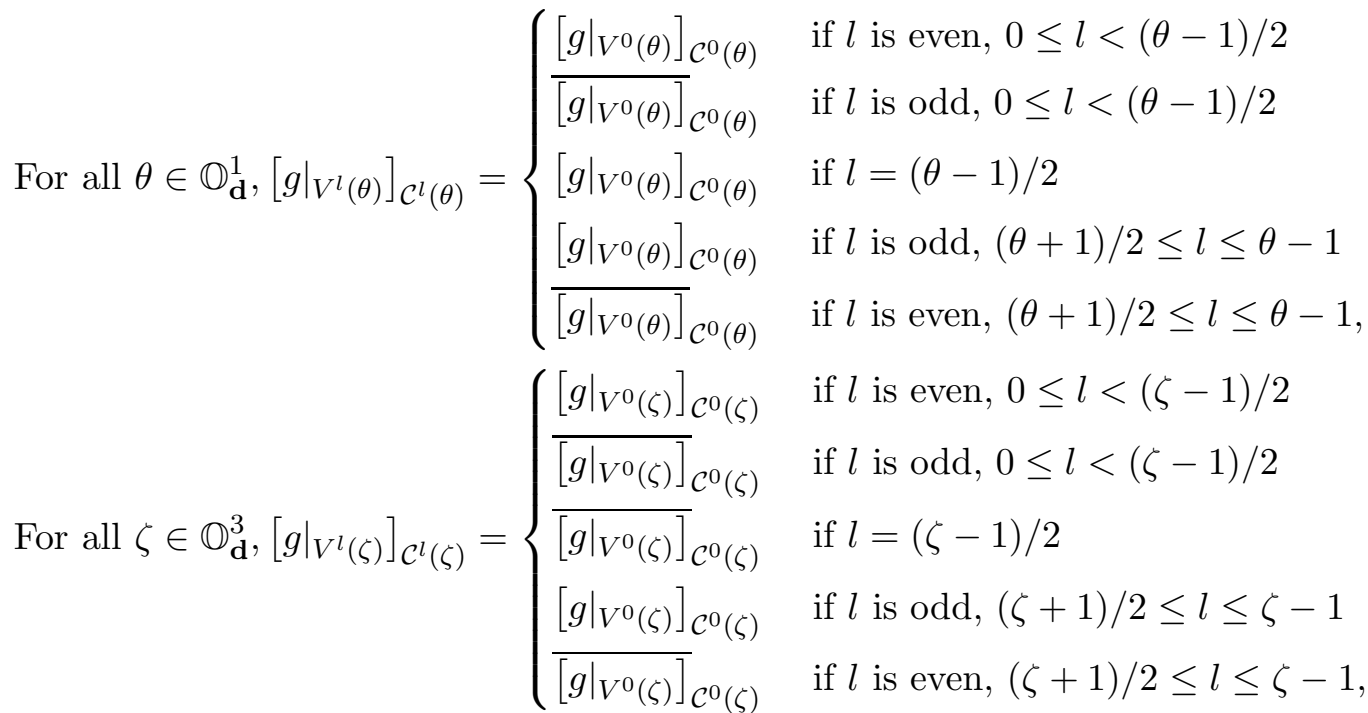

$\left.g\right|_{V^{0}(\theta)}$ commutes with $\mathrm{J}_{\mathcal{C}^{0}(\theta)}$,

$g\left(X^{l} L(\eta-1)\right) \subset X^{l} L(\eta-1),\left[\left.g\right|_{X^{l} L(\eta-1)}\right]_{\mathcal{B}^{l}(\eta)}=\left[\left.g\right|_{L(\eta-1)}\right]_{\mathcal{B}^{0}(\eta)}$ if $\eta \in \mathbb{E}_{\mathbf{d}}, 0 \leq l \leq \eta-1 ;$

$g\left(W^{l}(\eta)\right) \subset W^{l}(\eta)$ for $\eta \in \mathbb{E}_{\mathbf{d}}, 0 \leq l \leq \eta / 2-1$, and $\left.g\right|_{W^{0}(\eta)}$ commutes with $\mathrm{J}_{\mathcal{D}^{0}(\eta)}$.

Using Lemma 4.28(1) it is evident that $K^{\prime}$ is a maximal compact subgroup of $\mathcal{Z}_{\mathrm{SO}^{*}(2 n)}(X, H, Y)$. Hence to prove the lemma it suffices to show that $K=K^{\prime}$. Let $g \in \mathrm{SO}^{*}(2 n)$. From Lemma $4.28(2)$ it is straightforward that $g$ satisfies (1), (2), (3) of Lemma 4.29 if and only if $g$ satisfies (4.44), (4.45), (4.46) and (4.47). Now suppose that $g \in \mathrm{SO}^{*}(2 n)$ and $g$ satisfying (4), (5) of Lemma 4.29. It is clear that (4.49) holds. We observe that

$$
\left[\left.g\right|_{L(\eta-1)}\right]_{\mathcal{B}^{0}(\eta)}=\left(\begin{array}{cc}
A_{p_{\eta}}+\mathbf{j} B_{p_{\eta}}+\mathbf{i}\left(C_{p_{\eta}}+\mathbf{j} D_{p_{\eta}}\right) & 0 \\
0 & A_{q_{\eta}}^{\prime}+\mathbf{j} B_{q_{\eta}}^{\prime}+\mathbf{i}\left(C_{q_{\eta}}^{\prime}+\mathbf{j} D_{q_{\eta}}^{\prime}\right)
\end{array}\right)
$$

This proves that (4.48) holds.

Now we assume that $g$ satisfies (4.48) and (4.49). Let $A:=\left[\left.g\right|_{L(\eta-1)}\right]_{\mathcal{B}^{0}(\eta)}$. Then $A=$ $\left[\left.g\right|_{X^{l} L(\eta-1)}\right]_{\mathcal{B}^{l}(\eta)}$ for $1 \leq l \leq \eta-1$. We observe that

$$
\left[\mathrm{J}_{\mathcal{D}^{0}(\eta)}\right]_{\mathcal{B}^{0}(\eta) \vee \mathcal{B}^{\eta-1}(\eta)}=\left(\begin{array}{cc} 
& \mathrm{I}_{p_{\eta}, q_{\eta}} \\
-\mathrm{I}_{p_{\eta}, q_{\eta}} &
\end{array}\right) \text { and }\left[\left.g\right|_{W^{0}(\eta)}\right]_{\mathcal{B}^{0}(\eta) \vee \mathcal{B}^{\eta-1}(\eta)}=\left(\begin{array}{ll}
A & \\
& A
\end{array}\right) .
$$

From (4.49) it follows that the above two matrices commute, which in turn implies that $A$ commutes with $\left(\begin{array}{cc}\mathrm{I}_{p_{\eta}} & \\ & -\mathrm{I}_{q_{\eta}}\end{array}\right)$. Thus $A$ is of the form $A=\left(\begin{array}{cc}E_{p_{\eta}} & 0 \\ 0 & F_{q_{\eta}}\end{array}\right)$ for some matrices $E_{p_{\eta}} \in$ $\mathrm{GL}_{p_{\eta}}(\mathbb{H})$ and $F_{q_{\eta}} \in \mathrm{GL}_{q_{\eta}}(\mathbb{H})$. Write $E_{p_{\eta}}=A_{p_{\eta}}+\mathbf{j} B_{p_{\eta}}+\mathbf{i}\left(C_{p_{\eta}}+\mathbf{j} D_{p_{\eta}}\right)$ and $F_{q_{\eta}}=A_{q_{\eta}}^{\prime}+\mathbf{j} B_{q_{\eta}}^{\prime}+$ $\mathbf{i}\left(C_{q_{\eta}}^{\prime}+\mathbf{j} D_{q_{\eta}}^{\prime}\right)$ where $A_{p_{\eta}}, B_{p_{\eta}}, C_{p_{\eta}}, D_{p_{\eta}} \in \mathrm{M}_{p_{\eta}}(\mathbb{R}), A_{q_{\eta}}^{\prime}, B_{q_{\eta}}^{\prime}, C_{q_{\eta}}^{\prime}, D_{q_{\eta}}^{\prime} \in \mathrm{M}_{q_{\eta}}(\mathbb{R})$. We now observe 
that

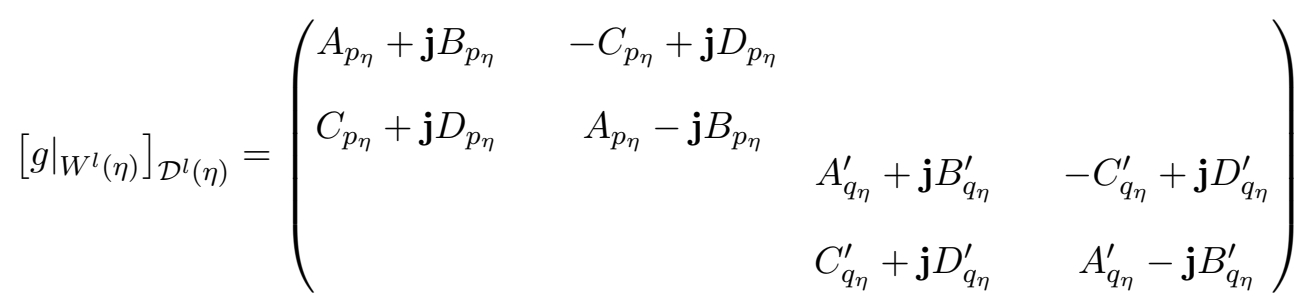

where $\mathcal{D}^{l}(\eta)$ is defined as in (4.41).

Recall that $M(\eta-1)=\bigoplus_{l=0}^{\eta / 2} W^{l}(\eta)$ is an orthogonal decomposition of $M(\eta-1)$ with respect to $\langle\cdot, \cdot\rangle$; see (4.40) and the paragraph preceding it. As $\mathcal{D}^{0}(\eta)$ is a standard orthogonal basis of $W^{0}(\eta)$, and $\left.g\right|_{W^{0}(\eta)}$ commutes with $\mathrm{J}_{\mathcal{D}^{0}(\eta)}$, it follows that $\Lambda_{\mathcal{D}^{0}(\eta)}^{\prime}\left(\left.g\right|_{W^{0}(\eta)}\right) \in \mathrm{U}\left(2 t_{\eta}\right)$. In other words,

$$
\left(\begin{array}{llll}
A_{p_{\eta}}+\sqrt{-1} B_{p_{\eta}} & -C_{p_{\eta}}+\sqrt{-1} D_{p_{\eta}} & & \\
C_{p_{\eta}}+\sqrt{-1} D_{p_{\eta}} & A_{p_{\eta}}-\sqrt{-1} B_{p_{\eta}} & & \\
& & A_{q_{\eta}}^{\prime}+\sqrt{-1} B_{q_{\eta}}^{\prime} & -C_{q_{\eta}}^{\prime}+\sqrt{-1} D_{q_{\eta}}^{\prime} \\
& & C_{q_{\eta}}^{\prime}+\sqrt{-1} D_{q_{\eta}}^{\prime} & A_{q_{\eta}}^{\prime}-\sqrt{-1} B_{q_{\eta}}^{\prime}
\end{array}\right) \in \mathrm{U}\left(2 t_{\eta}\right) .
$$

This implies that $E_{p_{\eta}} \in \operatorname{Sp}\left(p_{\eta}\right)$ and $F_{q_{\eta}} \in \operatorname{Sp}\left(q_{\eta}\right)$ and (5) of lemma (4.29) holds. This completes the proof.

We now introduce some notation which will be required to state Proposition 4.30. For $\eta \in \mathbb{E}_{\mathbf{d}}$, set

$$
\mathcal{D}(\eta):=\mathcal{D}^{0}(\eta) \vee \cdots \vee \mathcal{D}^{\eta / 2-1}(\eta)
$$

and for $\theta \in \mathbb{O}_{\mathbf{d}}$, set

$$
\mathcal{C}(\theta):=\mathcal{C}^{0}(\theta) \vee \cdots \vee \mathcal{C}^{\theta-1}(\theta)
$$

Let $\alpha:=\# \mathbb{E}_{\mathbf{d}}, \beta:=\# \mathbb{O}_{\mathbf{d}}^{1}$ and $\gamma:=\# \mathbb{O}_{\mathbf{d}}^{3}$. We enumerate $\mathbb{E}_{\mathbf{d}}=\left\{\eta_{i} \mid 1 \leq i \leq \alpha\right\}$ such that $\eta_{i}<\eta_{i+1}, \mathbb{O}_{\mathbf{d}}^{1}=\left\{\theta_{j} \mid 1 \leq j \leq \beta\right\}$ such that $\theta_{j}<\theta_{j+1}$ and similarly $\mathbb{O}_{\mathbf{d}}^{3}=\left\{\zeta_{j} \mid 1 \leq j \leq \gamma\right\}$ such that $\zeta_{j}<\zeta_{j+1}$. Now define

$$
\mathcal{E}:=\mathcal{D}\left(\eta_{1}\right) \vee \cdots \vee \mathcal{D}\left(\eta_{\alpha}\right) ; \mathcal{O}^{1}:=\mathcal{C}\left(\theta_{1}\right) \vee \cdots \vee \mathcal{C}\left(\theta_{\beta}\right) ; \text { and } \mathcal{O}^{3}:=\mathcal{C}\left(\zeta_{1}\right) \vee \cdots \vee \mathcal{C}\left(\zeta_{\gamma}\right)
$$

Also define

$$
\mathcal{H}=\mathcal{E} \vee \mathcal{O}^{1} \vee \mathcal{O}^{3}
$$

For an integer $m$ define the $\mathbb{R}$-algebra embedding

$$
\wp_{m, \mathbb{H}}: \mathrm{M}_{m}(\mathbb{H}) \longrightarrow \mathrm{M}_{2 m}(\mathbb{C}), \quad R \longmapsto\left(\begin{array}{cc}
S & -\bar{T} \\
T & \bar{S}
\end{array}\right)
$$

where $S, T \in \mathrm{M}_{m}(\mathbb{C})$ are the unique elements such that $R=S+\mathbf{j} T$. The following map is an $\mathbb{R}$-algebra embedding of $\prod_{i=1}^{\alpha}\left(\mathrm{M}_{p_{\eta_{i}}}(\mathbb{H}) \times \mathrm{M}_{q_{\eta_{i}}}(\mathbb{H})\right) \times \prod_{j=1}^{\beta} \mathrm{M}_{t_{\theta_{j}}}(\mathbb{C}) \times \prod_{k=1}^{\gamma} \mathrm{M}_{t_{\zeta_{k}}}(\mathbb{C})$ into $\mathrm{M}_{n}(\mathbb{C})$. Define

$$
\mathbf{D}: \prod_{i=1}^{\alpha}\left(\mathrm{M}_{p_{\eta_{i}}}(\mathbb{H}) \times \mathrm{M}_{q_{\eta_{i}}}(\mathbb{H})\right) \times \prod_{j=1}^{\beta} \mathrm{M}_{t_{\theta_{j}}}(\mathbb{C}) \times \prod_{k=1}^{\gamma} \mathrm{M}_{t_{\zeta_{k}}}(\mathbb{C}) \longrightarrow \mathrm{M}_{n}(\mathbb{C})
$$


by

$$
\begin{aligned}
\left(C_{\eta_{1}}, D_{\eta_{1}}, \cdots, C_{\eta_{\alpha}}, D_{\eta_{\alpha}} ; A_{\theta_{1}}, \cdots, A_{\theta_{\beta}} ; B_{\zeta_{1}}, \cdots, B_{\zeta_{\gamma}}\right) & \longmapsto \bigoplus_{i=1}^{\alpha}\left(\wp_{\eta_{i}, \mathbb{H}}\left(C_{\eta_{i}}\right) \oplus \wp_{\eta_{\eta_{i}}, \mathbb{H}}\left(D_{\eta_{i}}\right)\right)_{\mathbf{\Lambda}}^{\frac{\eta}{2}} \\
& \oplus \bigoplus_{j=1}^{\beta}\left(\left(A_{\theta_{j}} \oplus \bar{A}_{\theta_{j}}\right)^{\frac{\theta_{j}-1}{4}} \oplus A_{\theta_{j}} \oplus\left(A_{\theta_{j}} \oplus \bar{A}_{\theta_{j}}\right)^{\frac{\theta_{j}-1}{4}}\right) \\
& \oplus \bigoplus_{k=1}^{\gamma}\left(\left(B_{\zeta_{k}} \oplus \bar{B}_{\zeta_{k}}\right)^{\frac{\zeta_{k}+1}{4}} \oplus\left(B_{\zeta_{k}} \oplus \bar{B}_{\zeta_{k}}\right)^{\frac{\zeta_{k}-3}{4}} \oplus \bar{B}_{\zeta_{k}}\right) .
\end{aligned}
$$

It is clear that $\mathcal{H}$ in (4.50) is a standard orthogonal basis of $V$ with respect to $\langle\cdot, \cdot\rangle$. Let

$$
\Lambda_{\mathcal{H}}^{\prime}:\left\{x \in \operatorname{End}_{\mathbb{H}} \mathbb{H}^{n} \mid x \mathrm{~J}_{\mathcal{H}}=\mathrm{J}_{\mathcal{H}} x\right\} \longrightarrow \mathrm{M}_{n}(\mathbb{C})
$$

be the isomorphism of $\mathbb{R}$-algebras induced by the above ordered basis $\mathcal{H}$. Recall that $\Lambda_{\mathcal{H}}^{\prime}: K_{\mathcal{H}}$ $\longrightarrow \mathrm{U}(n)$ is an isomorphism of Lie groups.

Proposition 4.30. Let $X \in \mathcal{N}_{\mathfrak{s o}^{*}(2 n)}, \Psi_{\mathrm{SO}^{*}(2 n)}\left(\mathcal{O}_{X}\right)=\left(\mathbf{d}, \operatorname{sgn}_{\mathcal{O}_{X}}\right)$. Let $\alpha:=\# \mathbb{E}_{\mathbf{d}}, \beta:=\# \mathbb{O}_{\mathbf{d}}^{1}$ and $\gamma:=\# \mathbb{O}_{\mathbf{d}}^{3}$. Let $\{X, H, Y\} \subset \mathfrak{s o}^{*}(2 n)$ be a $\mathfrak{s l}_{2}(\mathbb{R})$-triple; let $\left(p_{\eta}, q_{\eta}\right)$ be the signature of the form $(\cdot, \cdot)_{\eta}$, for $\eta \in \mathbb{E}_{\mathbf{d}}$, as defined in (4.6). Let $K$ be the maximal compact subgroup of $\mathcal{Z}_{\mathrm{SO}^{*}(2 n)}(X, H, Y)$ as in Lemma 4.29. Then $\Lambda_{\mathcal{H}}^{\prime}(K) \subset \mathrm{U}(n)$ is given by

$$
\Lambda_{\mathcal{H}}^{\prime}(K)=\left\{\mathbf{D}(g) \mid g \in \prod_{i=1}^{\alpha}\left(\operatorname{Sp}\left(p_{\eta_{i}}\right) \times \operatorname{Sp}\left(q_{\eta_{i}}\right)\right) \times \prod_{j=1}^{\beta} \mathrm{U}\left(t_{\theta_{j}}\right) \times \prod_{k=1}^{\gamma} \mathrm{U}\left(t_{\zeta_{k}}\right)\right\} .
$$

Proof. This follows by writing the matrices of the elements of the maximal compact subgroup $K$ with respect to the basis $\mathcal{H}$ in $(4.50)$.

As we only consider simple Lie algebras, to ensure simplicity of $\mathfrak{s o}^{*}(2 n)$, in view of [Kn, Theorem 6.105, p. 421] and the isomorphisms (vii), (xi) in [He, Chapter X, §6, pp.519-520], we will further need to assume that $n \geq 3$.

Theorem 4.31. Let $X \in \mathfrak{s o}^{*}(2 n)$ be a nilpotent element when $n \geq 3$. Let $\left(\mathbf{d}, \mathbf{s g n}_{\mathcal{O}_{X}}\right) \in \mathcal{Y}^{\text {odd }}(n)$ be the signed Young diagram of the orbit $\mathcal{O}_{X}$ (that is, $\Psi_{\mathrm{SO}^{*}(2 n)}\left(\mathcal{O}_{X}\right)=\left(\mathbf{d}, \mathbf{s g n}_{\mathcal{O}_{X}}\right)$ in the notation of Theorem 4.27). Then

$$
\operatorname{dim}_{\mathbb{R}} H^{2}\left(\mathcal{O}_{X}, \mathbb{R}\right)= \begin{cases}0 & \text { if } \# \mathbb{O}_{\mathbf{d}}=0 \\ \# \mathbb{O}_{\mathbf{d}}-1 & \text { if } \# \mathbb{O}_{\mathbf{d}} \geq 1\end{cases}
$$

Proof. As the theorem is evident when $X=0$, we assume that $X \neq 0$.

In the proof we will use the notation established above. Let $\{X, H, Y\} \subset \mathfrak{s o}^{*}(2 n)$ be a $\mathfrak{s l}_{2}(\mathbb{R})$ triple. Let $K$ be the maximal compact subgroup of $\mathcal{Z}_{\mathrm{SO}^{*}(2 n)}(X, H, Y)$ as in Lemma 4.29. Let $\mathcal{H}$ be as in (4.50), and let $K_{\mathcal{H}}$ be the maximal compact subgroup of $\mathrm{SO}^{*}(2 n)$ as in the Lemma 4.28(1). Then $K \subset K_{\mathcal{H}}$. It follows either from Proposition 4.30 or from Lemma 4.4(4) that

$$
K \simeq \prod_{\eta \in \mathbb{E}_{\mathbf{d}}}\left(\operatorname{Sp}\left(p_{\eta}\right) \times \mathrm{Sp}\left(q_{\eta}\right)\right) \times \prod_{\theta \in \mathbb{O}_{\mathbf{d}}} \mathrm{U}\left(t_{\theta}\right) .
$$

In particular, $K$ is connected and $\operatorname{dim}_{\mathbb{R}} \mathfrak{z}(\mathfrak{k})=\# \mathbb{O}_{\mathbf{d}}$. Let $\mathfrak{k}_{\mathcal{H}}$ be the Lie algebra of $K_{\mathcal{H}}$. We now appeal to Proposition 4.30 to conclude that $\mathfrak{z}(\mathfrak{k}) \subset\left[\mathfrak{k}_{\mathcal{H}}, \mathfrak{k}_{\mathcal{H}}\right]$ when $\# \mathbb{O}_{\mathbf{d}}=0$, and $\mathfrak{z}(\mathfrak{k}) \not \subset\left[\mathfrak{k}_{\mathcal{H}}, \mathfrak{k}_{\mathcal{H}}\right]$ 
when $\# \mathbb{O}_{\mathbf{d}}>0$. As $\operatorname{dim}_{\mathbb{R}} \mathfrak{z}\left(\mathfrak{k}_{\mathcal{H}}\right)=1$, in view of Theorem 4.2 we have that for all $X \neq 0$,

$$
\operatorname{dim}_{\mathbb{R}} H^{2}\left(\mathcal{O}_{X}, \mathbb{R}\right)=\operatorname{dim}_{\mathbb{R}} \mathfrak{z}(\mathfrak{k}) \cap\left[\mathfrak{k}_{\mathcal{H}}, \mathfrak{k}_{\mathcal{H}}\right]= \begin{cases}0 & \text { if } \# \mathbb{O}_{\mathbf{d}}=0 \\ \# \mathbb{O}_{\mathbf{d}}-1 & \text { if } \# \mathbb{O}_{\mathbf{d}} \geq 1 .\end{cases}
$$

This completes the proof of the theorem.

4.7. Second cohomology groups of nilpotent orbits in $\mathfrak{s p}(n, \mathbb{R})$. Let $n$ be a positive integer. The aim in this subsection is to compute the second cohomology groups of nilpotent orbits in the simple real Lie algebra $\mathfrak{s p}(n, \mathbb{R})$ under the adjoint action of $\operatorname{Sp}(n, \mathbb{R})$. Throughout this subsection $\langle\cdot, \cdot\rangle$ denotes the symplectic form on $\mathbb{R}^{2 n}$ defined by $\langle x, y\rangle:=x^{t} \mathrm{~J}_{n} y, x, y \in \mathbb{R}^{2 n}$, where $J_{n}$ is as in (2.1). We will follow notation as defined in $\S 2$.

Let $\Psi_{\mathrm{SL}_{2 n}(\mathbb{R})}: \mathcal{N}\left(\mathrm{SL}_{2 n}(\mathbb{R})\right) \longrightarrow \mathcal{P}(2 n)$ be the parametrization of nilpotent orbits in $\mathfrak{s l} 2 n(\mathbb{R})$; see Theorem 4.6. As $\operatorname{Sp}(n, \mathbb{R}) \subset \mathrm{SL}_{2 n}(\mathbb{R})$ (consequently, $\mathcal{N}_{\mathfrak{s p}(n, \mathbb{R})} \subset \mathcal{N}_{\mathfrak{s l}_{2 n}(\mathbb{R})}$ ) we have the inclusion map $\Theta_{\mathrm{Sp}(n, \mathbb{R})}: \mathcal{N}(\mathrm{Sp}(n, \mathbb{R})) \longrightarrow \mathcal{N}\left(\mathrm{SL}_{2 n}(\mathbb{R})\right)$. Let

$$
\Psi_{\mathrm{Sp}(n, \mathbb{R})}^{\prime}:=\Psi_{\mathrm{SL}_{2 n}(\mathbb{R})} \circ \Theta_{\mathrm{Sp}(n, \mathbb{R})}: \mathcal{N}(\mathrm{Sp}(n, \mathbb{R})) \longrightarrow \mathcal{P}(2 n)
$$

be the composition. From Remark 5.16(1) it follows that $\Psi_{\operatorname{Sp}(n, \mathbb{R})}^{\prime}(\mathcal{N}(\operatorname{Sp}(n, \mathbb{R}))) \subset \mathcal{P}_{-1}(2 n)$. Let $0 \neq X \in \mathfrak{s p}(n, \mathbb{R})$ be a nilpotent element and $\mathcal{O}_{X}$ be the corresponding nilpotent orbit in $\mathfrak{s p}(n, \mathbb{R})$. Let $\{X, H, Y\} \subset \mathfrak{s p}(n, \mathbb{R})$ be a $\mathfrak{s l}_{2}(\mathbb{R})$-triple. We now apply Proposition 5.14, Remark 5.16 (1), and follow the notation used therein. Let $V:=\mathbb{R}^{2 n}$ be the right $\mathbb{R}$-vector space of column vectors. Let $\left\{d_{1}, \cdots, d_{s}\right\}$, with $d_{1}<\cdots<d_{s}$, be the ordered finite set of natural numbers that arise as dimension of non-zero irreducible $\operatorname{Span}_{\mathbb{R}}\{X, H, Y\}$-submodules of $V$. Recall that $M(d-1)$ is defined to be the isotypical component of $V$ containing all irreducible submodules of $V$ with highest weight $d-1$ and as in (2.2), we set $L(d-1):=V_{Y, 0} \cap M(d-1)$. Let $t_{d_{r}}:=\operatorname{dim}_{\mathbb{R}} L\left(d_{r}-1\right)$ for $1 \leq r \leq s$. Then $\mathbf{d}:=\left[d_{1}^{t_{d_{1}}}, \cdots, d_{s}^{t_{d_{s}}}\right] \in \mathcal{P}_{-1}(2 n)$, and moreover, $\Psi_{\mathrm{Sp}(n, \mathbb{R})}^{\prime}\left(\mathcal{O}_{X}\right)=\mathbf{d}$.

We next assign $\operatorname{sgn}_{\mathcal{O}_{X}} \in \mathcal{S}_{\mathbf{d}}^{\text {odd }}(2 n)$ to each $\mathcal{O}_{X} \in \mathcal{N}(\operatorname{Sp}(n, \mathbb{R}))$; see (2.9) for the definition of $\mathcal{S}_{\mathbf{d}}^{\text {odd }}(2 n)$. For each $d \in \mathbb{N}_{\mathbf{d}}$ (see (2.3) for the definition of $\mathbb{N}_{\mathbf{d}}$ ) we will define a $t_{d} \times d$ matrix $\left(m_{i j}^{d}\right)$ in $\mathbf{A}_{d}$ that depends only on the orbit $\mathcal{O}_{X}$; see (2.5) for the definition of $\mathbf{A}_{d}$. For this, recall that the form $(\cdot, \cdot)_{d}: L(d-1) \times L(d-1) \longrightarrow \mathbb{R}$ defined as in (4.6) is symmetric or symplectic according as $d$ is even or odd. Denoting the signature of $(\cdot, \cdot)_{\eta}$ by $\left(p_{\eta}, q_{\eta}\right)$ when $\eta \in \mathbb{E}_{\mathbf{d}}$, we now define

$$
\begin{aligned}
& m_{i 1}^{\theta}:=+1 \quad \text { if } 1 \leq i \leq t_{\theta}, \quad \theta \in \mathbb{O}_{\mathbf{d}} \\
& m_{i 1}^{\eta}:=\left\{\begin{array}{ll}
+1 & \text { if } 1 \leq i \leq p_{\eta} \\
-1 & \text { if } p_{\eta}<i \leq t_{\eta}
\end{array}, \quad \eta \in \mathbb{E}_{\mathbf{d}} ;\right.
\end{aligned}
$$

and for $j>1$, define $\left(m_{i j}^{d}\right)$ as in (4.15) and (4.16). Then the matrices $\left(m_{i j}^{d}\right)$ clearly verify (Yd.2). Set $\operatorname{sgn}_{\mathcal{O}_{X}}:=\left(\left(m_{i j}^{d_{1}}\right), \cdots,\left(m_{i j}^{d_{s}}\right)\right)$. It now follows from the above definition of $m_{i j}^{\theta}, \theta \in \mathbb{O}_{\mathbf{d}}$ that $\operatorname{sgn}_{\mathcal{O}_{X}} \in \mathcal{S}_{\mathbf{d}}^{\text {odd }}(2 n)$. Thus we have a map

$$
\Psi_{\mathrm{Sp}(n, \mathbb{R})}: \mathcal{N}(\operatorname{Sp}(n, \mathbb{R})) \longrightarrow \mathcal{Y}_{-1}^{\text {odd }}(2 n), \quad \mathcal{O}_{X} \longmapsto\left(\Psi_{\operatorname{Sp}(n, \mathbb{R})}^{\prime}\left(\mathcal{O}_{X}\right), \mathbf{s g n}_{\mathcal{O}_{X}}\right) ;
$$

where $\mathcal{Y}_{-1}^{\text {odd }}(2 n)$ is as in $(2.13)$.

Theorem 4.32 ([CoMc, Theorem 9.3.5]). The above map $\Psi_{\mathrm{Sp}(n, \mathbb{R})}$ is a bijection.

Let $0 \neq X \in \mathcal{N}_{\mathfrak{s p}(n, \mathbb{R})}$ and $\{X, H, Y\}$ be a $\mathfrak{s l}_{2}(\mathbb{R})$-triple in $\mathfrak{s p}(n, \mathbb{R})$. Let $\Psi_{\operatorname{Sp}(n, \mathbb{R})}\left(\mathcal{O}_{X}\right)=$ $\left(\mathbf{d}, \mathbf{s g n}_{\mathcal{O}_{X}}\right)$. Recall that $\mathbf{s g n}_{\mathcal{O}_{X}}$ determines the signature of $(\cdot, \cdot)_{\eta}$ on $L(\eta-1)$ for all $\eta \in \mathbb{E}_{\mathbf{d}}$; let $\left(p_{\eta}, q_{\eta}\right)$ be the signature of $(\cdot, \cdot)_{\eta}$ on $L(\eta-1)$. Let $\left(v_{1}^{d}, \cdots, v_{t_{d}}^{d}\right)$ be a $\mathbb{R}$-basis of $L(d-1)$ as in 
Proposition 5.14. It now follows from Proposition 5.14(3)(c) that $\left(v_{1}^{\eta}, \cdots, v_{t_{\eta}}^{\eta}\right)$ is an orthogonal basis of $L(\eta-1)$ for the form $(\cdot, \cdot)_{\eta}$. We also assume that the vectors in the basis $\left(v_{1}^{d}, \cdots, v_{t_{d}}^{d}\right)$ satisfy properties in Remark 5.16(1). In view of the signature of $(\cdot, \cdot)_{\eta}$, we may further assume that

$$
\left(v_{j}^{\eta}, v_{j}^{\eta}\right)_{\eta}=\left\{\begin{array}{ll}
+1 & \text { if } 1 \leq j \leq p_{\eta} \\
-1 & \text { if } p_{\eta}<j \leq t_{\eta}
\end{array} ; \eta \in \mathbb{E}_{\mathbf{d}} .\right.
$$

For all $\theta \in \mathbb{O}_{\mathbf{d}}$, as $(\cdot, \cdot)_{\theta}$ is a symplectic form, we may assume that $\left(v_{1}^{\theta}, \cdots, v_{t_{\theta} / 2}^{\theta} ; v_{t_{\theta} / 2+1}^{\theta}, \cdots\right.$, $\left.v_{t_{\theta}}^{\theta}\right)$ is a symplectic basis of $L(\theta-1)$; see Section 2.1 for the definition of a symplectic basis. This is equivalent to saying that, for all $\theta \in \mathbb{O}_{\mathbf{d}}$,

$$
\left(v_{j}^{\theta}, v_{t_{\theta} / 2+j}^{\theta}\right)_{\theta}=1 \text { for } 1 \leq j \leq t_{\theta} / 2 \text { and }\left(v_{j}^{\theta}, v_{i}^{\theta}\right)_{\theta}=0 \text { for all } i \neq j+t_{\theta} / 2 .
$$

Now fixing $\theta \in \mathbb{O}_{\mathbf{d}}$, for all $1 \leq j \leq t_{\theta}$, define

$$
w_{j l}^{\theta}:= \begin{cases}\left(X^{l} v_{j}^{\theta}+X^{\theta-1-l} v_{j}^{\theta}\right) \frac{1}{\sqrt{2}} & \text { if } 0 \leq l<(\theta-1) / 2 \\ X^{l} v_{j}^{\theta} & \text { if } l=(\theta-1) / 2 \\ \left(X^{\theta-1-l} v_{j}^{\theta}-X^{l} v_{j}^{\theta}\right) \frac{1}{\sqrt{2}} & \text { if }(\theta-1) / 2<l \leq \theta-1 .\end{cases}
$$

For $\theta \in \mathbb{O}_{\mathbf{d}}, 0 \leq l \leq \theta-1$, set

$$
V^{l}(\theta):=\operatorname{Span}_{\mathbb{R}}\left\{w_{j l}^{\theta} \mid 1 \leq j \leq t_{\theta}\right\} .
$$

The ordered basis $\left(w_{1 l}^{\theta}, \cdots, w_{t_{\theta} l}^{\theta}\right)$ of $V^{l}(\theta)$ is denoted by $\mathcal{A}^{l}(\theta)$. Let $\mathcal{B}^{l}(d)=\left(X^{l} v_{1}^{d}, \cdots, X^{l} v_{t_{d}}^{d}\right)$ be the ordered basis of $X^{l} L(d-1)$ for $0 \leq l \leq d-1, d \in \mathbb{N}_{\mathbf{d}}$ as in (4.3).

Lemma 4.33. The following holds:

$$
\mathcal{Z}_{\mathrm{Sp}(n, \mathbb{R})}(X, H, Y)=\left\{\begin{array}{c|c}
g\left(V^{l}(\theta)\right) \subset V^{l}(\theta) \text { and } \\
g \in \operatorname{Sp}(n, \mathbb{R}) \mid \begin{array}{c}
{\left[\left.g\right|_{V^{l}(\theta)}\right]_{\mathcal{A}^{l}(\theta)}=\left[\left.g\right|_{V^{0}(\theta)}\right]_{\mathcal{A}^{0}(\theta)} \text { for all } \theta \in \mathbb{O}_{\mathbf{d}}, 0 \leq l<\theta ;} \\
g\left(X^{l} L(\eta-1)\right) \subset X^{l} L(\eta-1) \text { and } \\
{\left[\left.g\right|_{X^{l} L(\eta-1)}\right]_{\mathcal{B}^{l}(\eta)}=\left[\left.g\right|_{L(\eta-1)}\right]_{\mathcal{B}^{0}(\eta)} \text { for all } \eta \in \mathbb{E}_{\mathbf{d}}, 0 \leq l<\eta}
\end{array}
\end{array}\right\} .
$$

Proof. The proof is similar to that of the Lemma 4.12; the details are omitted.

Using (4.52) and (4.53) we observe that for each $\theta \in \mathbb{O}_{\mathbf{d}}$ the space $M(\theta-1)$ is a direct sum of the subspaces $V^{l}(\theta), 0 \leq l \leq \theta-1$, which are mutually orthogonal with respect to $\langle\cdot, \cdot\rangle$. We now re-arrange the ordered basis $\mathcal{A}^{l}(\theta)$ of $V^{l}(\theta)$ to obtain a symplectic basis $\mathcal{C}^{l}(\theta)$ of $V^{l}(\theta)$ with respect to $\langle\cdot, \cdot\rangle$ as follows. For $\theta \in \mathbb{O}_{\mathbf{d}}^{1}$, define

$$
\mathcal{C}^{l}(\theta):= \begin{cases}\left(w_{1 l}^{\theta}, \cdots, w_{t_{\theta} / 2 l}^{\theta}\right) \vee\left(w_{\left(t_{\theta} / 2+1\right) l}^{\theta}, \cdots, w_{t_{\theta} l}^{\theta}\right) & \text { if } l \text { is even and } 0 \leq l<(\theta-1) / 2 \\ \left(w_{\left(t_{\theta} / 2+1\right) l}^{\theta}, \cdots, w_{t_{\theta} l}^{\theta}\right) \vee\left(w_{1 l}^{\theta}, \cdots, w_{t_{\theta} / 2 l}^{\theta}\right) & \text { if } l \text { is odd and } 0 \leq l<(\theta-1) / 2 \\ \left(w_{1 l}^{\theta}, \cdots, w_{t_{\theta} / 2 l}^{\theta}\right) \vee\left(w_{\left(t_{\theta} / 2+1\right) l}^{\theta}, \cdots, w_{t_{\theta} l}^{\theta}\right) & \text { if } l=(\theta-1) / 2 \\ \left(w_{\left(t_{\theta} / 2+1\right) l}^{\theta}, \cdots, w_{t_{\theta} l}^{\theta}\right) \vee\left(w_{1 l}^{\theta}, \cdots, w_{t_{\theta} / 2 l}^{\theta}\right) & \text { if } l \text { is even and }(\theta+1) / 2 \leq l \leq(\theta-1) \\ \left(w_{1 l}^{\theta}, \cdots, w_{t_{\theta} / 2 l}^{\theta}\right) \vee\left(w_{\left(t_{\theta} / 2+1\right) l}^{\theta}, \cdots, w_{t_{\theta} l}^{\theta}\right) & \text { if } l \text { is odd and }(\theta+1) / 2 \leq l \leq(\theta-1) .\end{cases}
$$


Similarly, for each $\zeta \in \mathbb{O}_{\mathbf{d}}^{3}$, define

$$
\mathcal{C}^{l}(\zeta):= \begin{cases}\left(w_{1 l}^{\zeta}, \cdots, w_{t_{\zeta} / 2 l}^{\zeta}\right) \vee\left(w_{\left(t_{\zeta} / 2+1\right) l}^{\zeta}, \cdots, w_{t_{\zeta} l}^{\zeta}\right) & \text { if } l \text { is even and } 0 \leq l<(\zeta-1) / 2 \\ \left(w_{\left(t_{\zeta} / 2+1\right) l}^{\zeta}, \cdots, w_{t_{\zeta} l}^{\zeta}\right) \vee\left(w_{1 l}^{\zeta}, \cdots, w_{t_{\zeta} / 2 l}^{\zeta}\right) & \text { if } l \text { is odd and } 0 \leq l<(\zeta-1) / 2 \\ \left(w_{\left(t_{\zeta} / 2+1\right) l}^{\zeta}, \cdots, w_{t_{\zeta} l}^{\zeta}\right) \vee\left(w_{1 l}^{\zeta}, \cdots, w_{t_{\zeta} / 2 l}^{\zeta}\right) & \text { if } l=(\zeta-1) / 2 \\ \left(w_{\left(t_{\zeta} / 2+1\right) l}^{\zeta}, \cdots, w_{t_{\zeta} l}^{\zeta}\right) \vee\left(w_{1 l}^{\zeta}, \cdots, w_{t_{\zeta} / 2 l}^{\zeta}\right) & \text { if } l \text { is even and }(\zeta+1) / 2 \leq l \leq(\zeta-1) \\ \left(w_{1 l}^{\zeta}, \cdots, w_{t_{\zeta} / 2 l}^{\zeta}\right) \vee\left(w_{\left(t_{\zeta} / 2+1\right) l}^{\zeta}, \cdots, w_{t_{\zeta} l}^{\zeta}\right) & \text { if } l \text { is odd and }(\zeta+1) / 2 \leq l \leq(\zeta-1) .\end{cases}
$$

For $\eta \in \mathbb{E}_{\mathbf{d}}, 0 \leq l \leq \eta / 2-1$, set

$$
W^{l}(\eta):=X^{l} L(\eta-1)+X^{\eta-1-l} L(\eta-1) .
$$

We moreover re-arrange the ordered basis $\mathcal{B}^{l}(\eta) \vee \mathcal{B}^{\eta-1-l}(\eta)$ of $W^{l}(\eta)$ and obtain new basis $\mathcal{D}^{l}(\eta)$ as follows:

$$
\mathcal{D}^{l}(\eta):= \begin{cases}\left(X^{l} v_{1}, \cdots, X^{l} v_{p_{\eta}}\right) \vee\left(X^{\eta-1-l} v_{p_{\eta}+1}, \cdots, X^{\eta-1-l} v_{t_{\eta}}\right) & \\ \bigvee\left(X^{\eta-1-l} v_{1}, \cdots, X^{\eta-1-l} v_{p_{\eta}}\right) \vee\left(X^{l} v_{p_{\eta}+1}, \cdots, X^{l} v_{t_{\eta}}\right) & \text { if } l \text { is even } \\ \left(X^{\eta-1-l} v_{1}, \cdots, X^{\eta-1-l} v_{p_{\eta}}\right) \vee\left(X^{l} v_{p_{\eta}+1}, \cdots, X^{l} v_{t_{\eta}}\right) & \\ \bigvee\left(X^{l} v_{1}, \cdots, X^{l} v_{p_{\eta}}\right) \vee\left(X^{\eta-1-l} v_{p_{\eta}+1}, \cdots, X^{\eta-1-l} v_{t_{\eta}}\right) & \text { if } l \text { is odd. }\end{cases}
$$

Using (4.51) it can be easily verified that $\mathcal{D}^{l}(\eta)$ is a symplectic basis with respect to $\langle\cdot, \cdot\rangle$.

Let $J_{\mathcal{C}^{l}(\theta)}$ be the complex structure on $V^{l}(\theta)$ associated to the basis $\mathcal{C}^{l}(\theta)$ for $\theta \in \mathbb{O}_{\mathbf{d}}, 0 \leq$ $l \leq \theta-1$, and let $J_{\mathcal{D}^{l}(\eta)}$ be the complex structure on $W^{l}(\eta)$ associated to the basis $\mathcal{D}^{l}(\eta)$ for $\eta \in \mathbb{E}_{\mathbf{d}}, 0 \leq l \leq \eta-1$; see Section 2.1 for the definition of such complex structures.

The next lemma is a standard fact where we recall, without a proof, an explicit description of a maximal compact subgroup in a symplectic group. Let $V^{\prime}$ be a $\mathbb{R}$-vector space, $\langle\cdot, \cdot\rangle^{\prime}$ be a non-degenerate symplectic form on $V^{\prime}$ and $\mathcal{B}^{\prime}$ be a symplectic basis of $V^{\prime}$. Let $J_{\mathcal{B}^{\prime}}$ be the complex structure on $V^{\prime}$ associated to $\mathcal{B}^{\prime}$. Let $2 m:=\operatorname{dim}_{\mathbb{R}} V^{\prime}$. We set

$$
K_{\mathcal{B}^{\prime}}:=\left\{g \in \operatorname{Sp}\left(V^{\prime},\langle\cdot, \cdot\rangle^{\prime}\right) \mid g J_{\mathcal{B}^{\prime}}=J_{\mathcal{B}^{\prime}} g\right\} .
$$

Lemma 4.34. Let $V^{\prime},\langle\cdot, \cdot\rangle^{\prime}, \mathcal{B}^{\prime}$ and $J_{\mathcal{B}^{\prime}}$ be as above. Then

(1) $K_{\mathcal{B}^{\prime}}$ is a maximal compact subgroup in $\operatorname{Sp}\left(V^{\prime},\langle\cdot, \cdot\rangle^{\prime}\right)$.

(2) $K_{\mathcal{B}^{\prime}}=\left\{g \in \mathrm{SL}\left(V^{\prime}\right) \mid[g]_{\mathcal{B}^{\prime}}=\left(\begin{array}{cc}A & -B \\ B & A\end{array}\right)\right.$ where $\left.A+\sqrt{-1} B \in \mathrm{U}(m)\right\}$.

Define the $\mathbb{R}$-algebra isomorphism

$$
\widetilde{\Lambda}_{\mathcal{B}^{\prime}}:\left\{x \in \operatorname{End}_{\mathbb{R}} V^{\prime} \mid x J_{\mathcal{B}^{\prime}}=J_{\mathcal{B}^{\prime}} x\right\} \longrightarrow \mathrm{M}_{m}(\mathbb{C}), \quad x \longmapsto A+\sqrt{-1} B
$$

where $[x]_{\mathcal{B}^{\prime}}=\left(\begin{array}{cc}A & -B \\ B & A\end{array}\right)$. In view of Lemma 4.34 it is clear that $\widetilde{\Lambda}_{\mathcal{B}^{\prime}}\left(K_{\mathcal{B}^{\prime}}\right)=\mathrm{U}(m)$, and thus $\widetilde{\Lambda}_{\mathcal{B}^{\prime}}: K_{\mathcal{B}^{\prime}} \rightarrow \mathrm{U}(m)$ is an isomorphism of Lie groups.

In the next lemma we describe a suitable maximal compact subgroup of $\mathcal{Z}_{\operatorname{Sp}(n, \mathbb{R})}(X, H, Y)$ which will be used in Proposition 4.36. Define the $\mathbb{R}$-algebra embedding

$$
\wp_{m, \mathbb{C}}: \mathrm{M}_{m}(\mathbb{C}) \longrightarrow \mathrm{M}_{2 m}(\mathbb{R}), \quad R \longmapsto\left(\begin{array}{cc}
S & -T \\
T & S
\end{array}\right)
$$

where $S, T \in \mathrm{M}_{m}(\mathbb{R})$ are the unique elements such that $R=S+\sqrt{-1} T$. 
Lemma 4.35. Let $K$ be the subgroup of $\mathcal{Z}_{\mathrm{Sp}(n, \mathbb{R})}(X, H, Y)$ consisting of elements $g$ in $\mathcal{Z}_{\mathrm{Sp}(n, \mathbb{R})}(X, H, Y)$ satisfying the following conditions:

(1) For all $\theta \in \mathbb{O}_{\mathbf{d}}$ and $0 \leq l \leq \theta-1, \quad g\left(V^{l}(\theta)\right) \subset V^{l}(\theta)$.

(2) For all $\theta \in \mathbb{O}_{\mathbf{d}}^{1}$, there exist $A_{\theta}, B_{\theta} \in \mathrm{M}_{t_{\theta} / 2}(\mathbb{R})$ with $A_{\theta}+\sqrt{-1} B_{\theta} \in \mathrm{U}\left(t_{\theta} / 2\right)$ such that

$$
\left[\left.g\right|_{V^{l}(\theta)}\right]_{\mathcal{C}^{l}(\theta)}= \begin{cases}\wp_{t_{\theta} / 2, \mathbb{C}}\left(A_{\theta}+\sqrt{-1} B_{\theta}\right) & \text { if } l \text { is even, } 0 \leq l<(\theta-1) / 2 \\ \wp_{t_{\theta} / 2, \mathbb{C}}\left(A_{\theta}-\sqrt{-1} B_{\theta}\right) & \text { if } l \text { is odd, } 0 \leq l<(\theta-1) / 2 \\ \wp_{t_{\theta} / 2, \mathbb{C}}\left(A_{\theta}+\sqrt{-1} B_{\theta}\right) & \text { if } l=(\theta-1) / 2 \\ \wp_{t_{\theta} / 2, \mathbb{C}}\left(A_{\theta}+\sqrt{-1} B_{\theta}\right) & \text { if } l \text { is odd, }(\theta+1) / 2 \leq l \leq \theta-1 \\ \wp_{t_{\theta} / 2, \mathbb{C}}\left(A_{\theta}-\sqrt{-1} B_{\theta}\right) & \text { if } l \text { is even, }(\theta+1) / 2 \leq l \leq \theta-1 .\end{cases}
$$

(3) For all $\zeta \in \mathbb{O}_{\mathbf{d}}^{3}$, there exist $A_{\zeta}, B_{\zeta} \in \mathrm{M}_{t_{\zeta} / 2}(\mathbb{R})$ with $A_{\zeta}+\sqrt{-1} B_{\zeta} \in \mathrm{U}\left(t_{\zeta} / 2\right)$ such that

$$
\left[\left.g\right|_{V^{l}(\zeta)}\right]_{\mathcal{C}^{l}(\zeta)}= \begin{cases}\wp_{t_{\zeta} / 2, \mathbb{C}}\left(A_{\zeta}+\sqrt{-1} B_{\zeta}\right) & \text { if } l \text { is even, } 0 \leq l<(\zeta-1) / 2 \\ \wp_{t_{\zeta} / 2, \mathbb{C}}\left(A_{\zeta}-\sqrt{-1} B_{\zeta}\right) & \text { if } l \text { is odd, } 0 \leq l<(\zeta-1) / 2 \\ \wp_{t_{\zeta} / 2, \mathbb{C}}\left(A_{\zeta}-\sqrt{-1} B_{\zeta}\right) & \text { if } l=(\zeta-1) / 2 \\ \wp_{t_{\zeta} / 2, \mathbb{C}}\left(A_{\zeta}+\sqrt{-1} B_{\zeta}\right) & \text { if } l \text { is odd, }(\zeta+1) / 2 \leq l \leq \zeta-1 \\ \wp_{t_{\zeta} / 2, \mathbb{C}}\left(A_{\zeta}-\sqrt{-1} B_{\zeta}\right) & \text { if } l \text { is even, }(\zeta+1) / 2 \leq l \leq \zeta-1 .\end{cases}
$$

(4) For all $\eta \in \mathbb{E}_{\mathbf{d}}$ and $0 \leq l \leq \eta-1, \quad g\left(X^{l} L(\eta-1)\right) \subset X^{l} L(\eta-1)$.

(5) For all $\eta \in \mathbb{E}_{\mathbf{d}}$, there exist $C_{\eta} \in \mathrm{O}_{p_{\eta}}$ and $D_{\eta} \in \mathrm{O}_{q_{\eta}}$ such that

$$
\left[\left.g\right|_{X^{l} L(\eta-1)}\right]_{\mathcal{B}^{l}(\eta)}=\left(\begin{array}{cc}
C_{\eta} & 0 \\
0 & D_{\eta}
\end{array}\right) \text {. }
$$

Then $K$ is a maximal compact subgroup of $\mathcal{Z}_{\mathrm{Sp}(n, \mathbb{R})}(X, H, Y)$.

Proof. For our convenience we begin by introducing a new notation. Let $m$ be an integer. For a matrix $Z$ in $\mathrm{M}_{2 m}(\mathbb{R})$, define

$$
Z^{\dagger}:=\left(\begin{array}{cc}
0 & \mathrm{I}_{m} \\
\mathrm{I}_{m} & 0
\end{array}\right) Z\left(\begin{array}{cc}
0 & \mathrm{I}_{m} \\
\mathrm{I}_{m} & 0
\end{array}\right)^{-1}
$$

Note that $\left(\begin{array}{cc}P & -R \\ R & P\end{array}\right)^{\dagger}=\left(\begin{array}{cc}P & R \\ -R & P\end{array}\right)$ for matrices $P, R \in \mathrm{M}_{m}(\mathbb{R})$. 
Let $K^{\prime} \subset \mathcal{Z}_{\mathrm{Sp}(n, \mathbb{R})}(X, H, Y)$ be the subgroup consisting of all elements $g$ satisfying the following conditions:

For all $\theta \in \mathbb{O}_{\mathbf{d}}, 0 \leq l \leq \theta-1, g\left(V^{l}(\theta)\right) \subset V^{l}(\theta)$.

For all $\theta \in \mathbb{O}_{\mathbf{d}}^{1},\left[\left.g\right|_{V^{l}(\theta)}\right]_{\mathcal{C}^{l}(\theta)}= \begin{cases}{\left[\left.g\right|_{V^{0}(\theta)}\right]_{\mathcal{C}^{0}(\theta)}} & \text { if } l \text { is even, } 0 \leq l<(\theta-1) / 2 \\ {\left[\left.g\right|_{V^{0}(\theta)}\right]_{\mathcal{C}^{0}(\theta)}^{\dagger}} & \text { if } l \text { is odd, } 0 \leq l<(\theta-1) / 2 \\ {\left[\left.g\right|_{V^{0}(\theta)}\right]_{\mathcal{C}^{0}(\theta)}} & \text { if } l=(\theta-1) / 2 \\ {\left[\left.g\right|_{V^{0}(\theta)}\right]_{\mathcal{C}^{0}(\theta)}} & \text { if } l \text { is odd, }(\theta+1) / 2 \leq l \leq \theta-1 \\ {\left[\left.g\right|_{V^{0}(\theta)}\right]_{\mathcal{C}^{0}(\theta)}^{\dagger}} & \text { if } l \text { is even, }(\theta+1) / 2 \leq l \leq \theta-1,\end{cases}$

For all $\zeta \in \mathbb{O}_{\mathbf{d}}^{3},\left[\left.g\right|_{V^{l}(\zeta)}\right]_{\mathcal{C}^{l}(\zeta)}= \begin{cases}{\left[\left.g\right|_{V^{0}(\zeta)}\right]_{\mathcal{C}^{0}(\zeta)}} & \text { if } l \text { is even, } 0 \leq l<(\zeta-1) / 2 \\ {\left[\left.g\right|_{V^{0}(\zeta)}\right]_{\mathcal{C}^{0}(\zeta)}^{\dagger}} & \text { if } l \text { is odd, } 0 \leq l<(\zeta-1) / 2 \\ {\left[\left.g\right|_{V^{0}(\zeta)}\right]_{\mathcal{C}^{0}(\zeta)}^{\dagger}} & \text { if } l=(\zeta-1) / 2 \\ {\left[\left.g\right|_{V^{0}(\zeta)}\right]_{\mathcal{C}^{0}(\zeta)}} & \text { if } l \text { is odd, }(\zeta+1) / 2 \leq l \leq \zeta-1 \\ {\left[\left.g\right|_{V^{0}(\zeta)}\right]_{\mathcal{C}^{0}(\zeta)}^{\dagger}} & \text { if } l \text { is even, }(\zeta+1) / 2 \leq l \leq \zeta-1,\end{cases}$ $\left.g\right|_{V^{0}(\theta)}$ commutes with $J_{\mathcal{C}^{0}(\theta)}$,

$g\left(X^{l} L(\eta-1)\right) \subset X^{l} L(\eta-1),\left[\left.g\right|_{X^{l} L(\eta-1)}\right]_{\mathcal{B}^{l}(\eta)}=\left[\left.g\right|_{L(\eta-1)}\right]_{\mathcal{B}^{0}(\eta)}$ for all $\eta \in \mathbb{E}_{\mathbf{d}}$, $0 \leq l \leq \eta-1$, $\left.g\right|_{W^{0}(\eta)}$ commutes with $J_{\mathcal{D}^{0}(\eta)}$.

Using Lemma 4.33 and Lemma 4.34(1) it is clear that $K^{\prime}$ is a maximal compact subgroup of $\mathcal{Z}_{\mathrm{Sp}(n, \mathbb{R})}(X, H, Y)$. Hence to prove the lemma it suffices to show that $K=K^{\prime}$. Let $g \in \operatorname{Sp}(n, \mathbb{R})$. Using Lemma 4.34 (2) it is straightforward to check that $g$ satisfies (1), (2), (3) in the statement of the lemma if and only if $g$ satisfies (4.58), (4.59), (4.60) and (4.61). Now suppose that $g \in \operatorname{Sp}(n, \mathbb{R})$ and $g$ satisfies (4) and (5) in the statement of the lemma. It is clear that (4.62) holds. We observe that

$$
\left[J_{\mathcal{D}^{0}(\eta)}\right]_{\mathcal{D}^{0}(\eta)}=\left(\begin{array}{cc}
0 & -\mathrm{I}_{t_{\eta}} \\
\mathrm{I}_{t_{\eta}} & 0
\end{array}\right) \text { and }\left[\left.g\right|_{W^{0}(\eta)}\right]_{\mathcal{D}^{0}(\eta)}=\left(\begin{array}{cccc}
C_{\eta} & & & \\
& D_{\eta} & & \\
& & C_{\eta} & \\
& & & D_{\eta}
\end{array}\right)
$$

where $\mathcal{D}^{0}(\eta)$ is defined by setting $l=0$ in (4.56). From the matrix representations as above, it is clear that $J_{\mathcal{D}^{0}(\eta)}$ and $\left.g\right|_{W^{0}(\eta)}$ commute. This proves that (4.63) holds.

Now we assume that $g$ satisfies (4.62) and (4.63). It is clear that (4) in the statement of the lemma holds. Note that $A:=\left[\left.g\right|_{L(\eta-1)}\right]_{\mathcal{B}^{0}(\eta)}=\left[\left.g\right|_{X^{l} L(\eta-1)}\right]_{\mathcal{B}^{l}(\eta)}$ for $1 \leq l \leq \eta-1$. We observe that

$$
\left[J_{\mathcal{D}^{0}(\eta)}\right]_{\mathcal{B}^{0}(\eta) \vee \mathcal{B}^{\eta-1}(\eta)}=\left(\begin{array}{cc}
0 & -\mathrm{I}_{p_{\eta}, q_{\eta}} \\
\mathrm{I}_{p_{\eta}, q_{\eta}} & 0
\end{array}\right) \text { and }\left[\left.g\right|_{W^{0}(\eta)}\right]_{\mathcal{B}^{0}(\eta) \vee \mathcal{B}^{\eta-1}(\eta)}=\left(\begin{array}{ll}
A & \\
& A
\end{array}\right)
$$

From (4.63) it follows that the above two matrices commute, which in turn implies that $A$ commutes with $\left(\begin{array}{cc}\mathrm{I}_{p_{\eta}} & \\ & -\mathrm{I}_{q_{\eta}}\end{array}\right)$. Thus $A$ is of the form $A=\left(\begin{array}{cc}C & 0 \\ 0 & D\end{array}\right)$ for some matrices $C \in$ 
$\mathrm{GL}_{p_{\eta}}(\mathbb{R})$ and $D \in \mathrm{GL}_{q_{\eta}}(\mathbb{R})$. Now observe that

$$
\left[\left.g\right|_{W^{0}(\eta)}\right]_{\mathcal{D}^{0}(\eta)}=\left(\begin{array}{llll}
C & & & \\
& D & & \\
& & C & \\
& & & D
\end{array}\right) .
$$

As $\left.g\right|_{W^{0}(\eta)}$ commutes with $J_{\mathcal{D}^{0}(\eta)}$, it follows that

$$
\left(\begin{array}{cc}
C & 0 \\
0 & D
\end{array}\right)+\sqrt{-1}\left(\begin{array}{ll}
0 & 0 \\
0 & 0
\end{array}\right) \in \mathrm{U}\left(t_{\eta}\right)
$$

Thus, $C \in \mathrm{O}_{p_{\eta}}$ and $D \in \mathrm{O}_{q_{\eta}}$ and (5) in the statement of the lemma holds. This completes the proof.

We next introduce some notation which will be needed in Proposition 4.36. Recall that the positive parts of the symplectic basis $\mathcal{D}(\eta), \mathcal{C}(\theta)$ are denoted by $\mathcal{D}_{+}(\eta), \mathcal{C}_{+}(\theta)$ respectively; see Section 2.1. Similarly, the negative parts of $\mathcal{D}(\eta), \mathcal{C}(\theta)$ are denoted by $\mathcal{D}_{-}(\eta), \mathcal{C}_{-}(\theta)$ respectively. For $\eta \in \mathbb{E}_{\mathbf{d}}$, set

$$
\mathcal{D}_{+}(\eta):=\mathcal{D}_{+}^{0}(\eta) \vee \cdots \vee \mathcal{D}_{+}^{\eta / 2-1}(\eta) \text { and } \mathcal{D}_{-}(\eta):=\mathcal{D}_{-}^{0}(\eta) \vee \cdots \vee \mathcal{D}_{-}^{\eta / 2-1}(\eta)
$$

For $\theta \in \mathbb{O}_{\mathbf{d}}$, set

$$
\mathcal{C}_{+}(\theta):=\mathcal{C}_{+}^{0}(\theta) \vee \cdots \vee \mathcal{C}_{+}^{\theta-1}(\theta) \text { and } \mathcal{C}_{-}(\theta):=\mathcal{C}_{-}^{0}(\theta) \vee \cdots \vee \mathcal{C}_{-}^{\theta-1}(\theta)
$$

Let $\alpha:=\# \mathbb{E}_{\mathbf{d}}, \beta:=\# \mathbb{O}_{\mathbf{d}}^{1}$ and $\gamma:=\# \mathbb{O}_{\mathbf{d}}^{3}$. We enumerate $\mathbb{E}_{\mathbf{d}}=\left\{\eta_{i} \mid 1 \leq i \leq \alpha\right\}$ such that $\eta_{i}<\eta_{i+1}$, and $\mathbb{O}_{\mathbf{d}}^{1}=\left\{\theta_{j} \mid 1 \leq j \leq \beta\right\}$ such that $\theta_{j}<\theta_{j+1}$; similarly enumerate $\mathbb{O}_{\mathbf{d}}^{3}=\left\{\zeta_{j} \mid 1 \leq j \leq \gamma\right\}$ such that $\zeta_{j}<\zeta_{j+1}$. Now define

$$
\begin{aligned}
& \mathcal{E}_{+}:=\mathcal{D}_{+}\left(\eta_{1}\right) \vee \cdots \vee \mathcal{D}_{+}\left(\eta_{\alpha}\right) ; \quad \mathcal{O}_{+}^{1}:=\mathcal{C}_{+}\left(\theta_{1}\right) \vee \cdots \vee \mathcal{C}_{+}\left(\theta_{\beta}\right) ; \quad \mathcal{O}_{+}^{3}:=\mathcal{C}_{+}\left(\zeta_{1}\right) \vee \cdots \vee \mathcal{C}_{+}\left(\zeta_{\gamma}\right) ; \\
& \mathcal{E}_{-}:=\mathcal{D}_{-}\left(\eta_{1}\right) \vee \cdots \vee \mathcal{D}_{-}\left(\eta_{\alpha}\right) ; \mathcal{O}_{-}^{1}:=\mathcal{C}_{-}\left(\theta_{1}\right) \vee \cdots \vee \mathcal{C}_{-}\left(\theta_{\beta}\right) \text { and } \mathcal{O}_{-}^{3}:=\mathcal{C}_{-}\left(\zeta_{1}\right) \vee \cdots \vee \mathcal{C}_{-}\left(\zeta_{\gamma}\right) .
\end{aligned}
$$

Also we define

$$
\mathcal{H}_{+}:=\mathcal{E}_{+} \vee \mathcal{O}_{+}^{1} \vee \mathcal{O}_{+}^{3}, \quad \mathcal{H}_{-}:=\mathcal{E}_{-} \vee \mathcal{O}_{-}^{1} \vee \mathcal{O}_{-}^{3} \text { and } \mathcal{H}:=\mathcal{H}_{+} \vee \mathcal{H}_{-}
$$

As before, for a matrix $A=\left(a_{i j}\right) \in \mathrm{M}_{r}(\mathbb{C})$, define $\bar{A}:=\left(\bar{a}_{i j}\right) \in \mathrm{M}_{r}(\mathbb{C})$. Let

$$
\mathbf{D}: \prod_{i=1}^{\alpha}\left(\mathrm{M}_{p_{\eta_{i}}}(\mathbb{R}) \times \mathrm{M}_{q_{\eta_{i}}}(\mathbb{R})\right) \times \prod_{j=1}^{\beta} \mathrm{M}_{t_{\theta_{j}} / 2}(\mathbb{C}) \times \prod_{k=1}^{\gamma} \mathrm{M}_{t_{\zeta_{k}} / 2}(\mathbb{C}) \longrightarrow \mathrm{M}_{n}(\mathbb{C})
$$

be the $\mathbb{R}$-algebra embedding defined by

$$
\begin{aligned}
\left(C_{\eta_{1}}, D_{\eta_{1}}, \cdots, C_{\eta_{\alpha}}, D_{\eta_{\alpha}} ; A_{\theta_{1}}, \cdots, A_{\theta_{\beta}} ; B_{\zeta_{1}}, \cdots, B_{\zeta_{\gamma}}\right) & \\
& \longmapsto \bigoplus_{i=1}^{\alpha}\left(C_{\eta_{i}} \oplus D_{\eta_{i}}\right)_{\mathbf{\Lambda}}^{\eta_{i} / 2} \oplus \bigoplus_{j=1}^{\beta}\left(\left(A_{\theta_{j}} \oplus \bar{A}_{\theta_{j}}\right)^{\frac{\theta_{j}-1}{4}} \oplus A_{\theta_{j}} \oplus\left(A_{\theta_{j}} \oplus \bar{A}_{\theta_{j}}\right)^{\frac{\theta_{j}-1}{4}}\right) \\
& \oplus \bigoplus_{k=1}^{\gamma}\left(\left(B_{\zeta_{k}} \oplus \bar{B}_{\zeta_{k}}\right)^{\frac{\zeta_{k}+1}{4}} \oplus\left(B_{\zeta_{k}} \oplus \bar{B}_{\zeta_{k}}\right)^{\frac{\zeta_{k}-3}{4}} \oplus \bar{B}_{\zeta_{k}}\right) .
\end{aligned}
$$

It is clear that the basis $\mathcal{H}$ in (4.64) is a symplectic basis of $V$ with respect to $\langle\cdot, \cdot\rangle$. Let $\widetilde{\Lambda}_{\mathcal{H}}:\left\{x \in \operatorname{End}_{\mathbb{R}} \mathbb{R}^{2 n} \mid x J_{\mathcal{H}}=J_{\mathcal{H}} x\right\} \longrightarrow \mathrm{M}_{n}(\mathbb{C})$ be the isomorphism of $\mathbb{R}$-algebras induced by the above symplectic basis $\mathcal{H}$. Recall that $\widetilde{\Lambda}_{\mathcal{H}}: K_{\mathcal{H}} \longrightarrow \mathrm{U}(n)$ is an isomorphism of Lie groups. Using (4.61) and (4.63) we observe that the group $K$ defined in Lemma 4.35 satisfies the condition $K \subset K_{\mathcal{H}}$. 
Proposition 4.36. Let $X \in \mathcal{N}_{\mathfrak{s p}(n, \mathbb{R})}$ and $\Psi_{\mathrm{Sp}(n, \mathbb{R})}\left(\mathcal{O}_{X}\right)=\left(\mathbf{d}, \operatorname{sgn}_{\mathcal{O}_{X}}\right)$. Let $\alpha:=\# \mathbb{E}_{\mathbf{d}}$, $\beta:=\# \mathbb{O}_{\mathbf{d}}^{1}$ and $\gamma:=\# \mathbb{O}_{\mathbf{d}}^{3}$. Let $\{X, H, Y\}$ be a $\mathfrak{s l}_{2}(\mathbb{R})$-triple in $\mathfrak{s p}(n, \mathbb{R})$, and let $\left(p_{\eta}, q_{\eta}\right)$ be the signature of $(\cdot, \cdot)_{\eta}, \eta \in \mathbb{E}_{\mathbf{d}}$, as defined in (4.6). Let $K$ be the maximal compact subgroup of $\mathcal{Z}_{\mathrm{Sp}(n, \mathbb{R})}(X, H, Y)$ as in Lemma 4.35. Then $\widetilde{\Lambda}_{\mathcal{H}}(K) \subset \mathrm{U}(n)$ is given by

$$
\widetilde{\Lambda}_{\mathcal{H}}(K)=\left\{\mathbf{D}(g) \mid g \in \prod_{i=1}^{\alpha}\left(\mathrm{O}_{p_{\eta_{i}}} \times \mathrm{O}_{q_{\eta_{i}}}\right) \times \prod_{j=1}^{\beta} \mathrm{U}\left(t_{\theta_{j}} / 2\right) \times \prod_{k=1}^{\gamma} \mathrm{U}\left(t_{\zeta_{k}} / 2\right)\right\} .
$$

Proof. This follows by writing the matrices of the elements of the maximal compact subgroup $K$ in Lemma 4.35 with respect to the symplectic basis $\mathcal{H}$ in (4.64).

Theorem 4.37. Let $X \in \mathfrak{s p}(n, \mathbb{R})$ be a nilpotent element. Let $\left(\mathbf{d}, \operatorname{sgn}_{\mathcal{O}_{X}}\right) \in \mathcal{Y}_{-1}^{\text {odd }}(2 n)$ be the signed Young diagram of the orbit $\mathcal{O}_{X}$ (that is, $\Psi_{\mathrm{Sp}(n, \mathbb{R})}\left(\mathcal{O}_{X}\right)=\left(\mathbf{d}, \mathbf{s g n}_{\mathcal{O}_{X}}\right)$ as in the notation of Theorem 4.32). Then

$$
\operatorname{dim}_{\mathbb{R}} H^{2}\left(\mathcal{O}_{X}, \mathbb{R}\right)= \begin{cases}0 & \text { if } \# \mathbb{O}_{\mathbf{d}}=0 \\ \# \mathbb{O}_{\mathbf{d}}-1 & \text { if } \# \mathbb{O}_{\mathbf{d}} \geq 1\end{cases}
$$

Proof. As the theorem is evident when $X=0$ we assume that $X \neq 0$.

Let $\{X, H, Y\} \subset \mathfrak{s p}(n, \mathbb{R})$ be a $\mathfrak{s l}_{2}(\mathbb{R})$-triple. Let $K$ be the maximal compact subgroup of $\mathcal{Z}_{\mathrm{Sp}(n, \mathbb{R})}(X, H, Y)$ as in Lemma 4.35 . Let $\mathcal{H}$ be as in (4.64) and $K_{\mathcal{H}}$ the maximal compact subgroup of $\operatorname{Sp}(n, \mathbb{R})$ as in Lemma $4.34(1)$. Then $K \subset K_{\mathcal{H}}$. Let $\mathfrak{k}_{\mathcal{H}}$ be the Lie algebra of $K_{\mathcal{H}}$. Using Proposition 4.36 it follows that $\mathfrak{z}(\mathfrak{k}) \subset\left[\mathfrak{k}_{\mathcal{H}}, \mathfrak{k}_{\mathcal{H}}\right]$ when $\# \mathbb{O}_{\mathbf{d}}=0$, and $\mathfrak{z}(\mathfrak{k}) \not \subset\left[\mathfrak{k}_{\mathcal{H}}, \mathfrak{k}_{\mathcal{H}}\right]$ when $\# \mathbb{O}_{\mathbf{d}} \geq 1$. As $\operatorname{dim}_{\mathbb{R}} \mathfrak{z}\left(\mathfrak{k}_{\mathcal{H}}\right)=1$, it follows that

$$
\operatorname{dim}_{\mathbb{R}} \mathfrak{z}(\mathfrak{k}) \cap\left[\mathfrak{k}_{\mathcal{H}}, \mathfrak{k}_{\mathcal{H}}\right]=\operatorname{dim}_{\mathbb{R}} \mathfrak{z}(\mathfrak{k})-1
$$

when $\# \mathbb{O}_{\mathbf{d}} \geq 1$. The group $\mathrm{O}_{2} / \mathrm{SO}_{2}=\mathbb{Z} / 2 \mathbb{Z}$ acts non-trivially on $\mathfrak{s o}_{2}$ and the group $\mathrm{U}(\mathrm{m})$ acts trivially on $\mathfrak{z}(\mathfrak{u}(m))$. We next use the observation in (4.12) to conclude that

$$
\operatorname{dim}_{\mathbb{R}}\left[\mathfrak{z}(\mathfrak{k}) \cap\left[\mathfrak{k}_{\mathcal{H}}, \mathfrak{k}_{\mathcal{H}}\right]\right]^{K / K^{\circ}}= \begin{cases}0 & \text { if } \# \mathbb{O}_{\mathbf{d}}=0 \\ \# \mathbb{O}_{\mathbf{d}}-1 & \text { if } \# \mathbb{O}_{\mathbf{d}} \geq 1 .\end{cases}
$$

Now the theorem follows from Theorem 4.2.

4.8. Second cohomology groups of nilpotent orbits in $\mathfrak{s p}(p, q)$. Let $n$ be a positive integer and $(p, q)$ be a pair of non-negative integers with $p+q=n$. In this subsection we compute the second cohomology groups of nilpotent orbits in $\mathfrak{s p}(p, q)$ under the adjoint action of $\operatorname{Sp}(p, q)$. As we do not need to deal with compact groups, we will further assume that $p>0$ and $q>0$. Throughout this subsection $\langle\cdot, \cdot\rangle$ denotes the Hermitian form on $\mathbb{H}^{n}$ defined by $\langle x, y\rangle:=\bar{x}^{t} \mathrm{I}_{p, q} y$, $x, y \in \mathbb{H}^{n}$, where $\mathrm{I}_{p, q}$ is as in (2.1). We will follow notation as defined in $\S 2$.

Let $\Psi_{\mathrm{SL}_{n}(\mathbb{H})}: \mathcal{N}\left(\mathrm{SL}_{n}(\mathbb{H})\right) \longrightarrow \mathcal{P}(n)$ be the parametrization as in Theorem 4.8. As $\operatorname{Sp}(p, q) \subset$ $\mathrm{SL}_{n}(\mathbb{H})$ (consequently, $\left.\mathcal{N}_{\mathfrak{s p}(p, q)} \subset \mathcal{N}_{\mathfrak{s l}_{n}(\mathbb{H})}\right)$ we have the inclusion map $\Theta_{\mathrm{Sp}(p, q)}: \mathcal{N}(\operatorname{Sp}(p, q)) \longrightarrow$ $\mathcal{N}\left(\mathrm{SL}_{n}(\mathbb{H})\right)$. Let

$$
\Psi_{\mathrm{Sp}(p, q)}^{\prime}:=\Psi_{\mathrm{SL}_{n}(\mathbb{H})} \circ \Theta_{\mathrm{Sp}(p, q)}: \mathcal{N}(\mathrm{Sp}(p, q)) \longrightarrow \mathcal{P}(n)
$$

be the composition. Let $0 \neq X \in \mathfrak{s p}(p, q)$ be a nilpotent element and $\mathcal{O}_{X}$ be the corresponding nilpotent orbit in $\mathfrak{s p}(p, q)$. Let $\{X, H, Y\} \subset \mathfrak{s p}(p, q)$ be a $\mathfrak{s l}_{2}(\mathbb{R})$-triple. We now apply Proposition 5.14, Remark 5.16(3), and follow the notation used therein. Let $V:=\mathbb{H}^{n}$ be the right $\mathbb{H}$-vector space of column vectors. Let $\left\{d_{1}, \cdots, d_{s}\right\}$, with $d_{1}<\cdots<d_{s}$, be a ordered finite subset of natural numbers that arise as $\mathbb{R}$-dimension of non-zero irreducible $\operatorname{Span}_{\mathbb{R}}\{X, H, Y\}$ submodules of $V$. Recall that $M(d-1)$ is defined to be the isotypical component of $V$ containing all irreducible $\operatorname{Span}_{\mathbb{R}}\{X, H, Y\}$-submodules of $V$ with highest weight $(d-1)$, and as in (2.2), we 
set $L(d-1):=V_{Y, 0} \cap M(d-1)$. Recall that the space $L\left(d_{r}-1\right)$ is a $\mathbb{H}$-subspace for $1 \leq r \leq s$. Let $t_{d_{r}}:=\operatorname{dim}_{\mathbb{H}} L\left(d_{r}-1\right)$ for $1 \leq r \leq s$. Then $\mathbf{d}:=\left[d_{1}^{t_{d_{1}}}, \cdots, d_{s}^{t_{d_{s}}}\right] \in \mathcal{P}(n)$, and moreover, $\Psi_{\operatorname{Sp}(p, q)}^{\prime}\left(\mathcal{O}_{X}\right)=\mathbf{d}$.

We next assign $\operatorname{sgn}_{\mathcal{O}_{X}} \in \mathcal{S}_{\mathbf{d}}^{\text {even }}(p, q)$ to each $\mathcal{O}_{X} \in \mathcal{N}(\operatorname{Sp}(p, q))$; see (2.8) for the definition of $\mathcal{S}_{\mathbf{d}}^{\text {even }}(p, q)$. For each $d \in \mathbb{N}_{\mathbf{d}}$ (see $(2.3)$ for the definition of $\mathbb{N}_{\mathbf{d}}$ ) we will define a $t_{d} \times d$ matrix $\left(m_{i j}^{d}\right)$ in $\mathbf{A}_{d}$ which depends only on the orbit $\mathcal{O}_{X}$ containing $X$; see (2.5) for the definition of $\mathbf{A}_{d}$. For this, recall that the form $(\cdot, \cdot)_{d}: L(d-1) \times L(d-1) \longrightarrow \mathbb{H}$ defined as in (4.6) is Hermitian or skew-Hermitian according as $d$ is odd or even. Denoting the signature of $(\cdot, \cdot)_{\theta}$ by $\left(p_{\theta}, q_{\theta}\right)$ when $\theta \in \mathbb{O}_{\mathbf{d}}$, we now define

$$
\begin{aligned}
& m_{i 1}^{\eta}:=+1 \quad \text { if } 1 \leq i \leq t_{\eta}, \quad \eta \in \mathbb{E}_{\mathbf{d}} ; \\
& m_{i 1}^{\theta}:=\left\{\begin{array}{ll}
+1 & \text { if } 1 \leq i \leq p_{\theta} \\
-1 & \text { if } p_{\theta}<i \leq t_{\theta}
\end{array}, \theta \in \mathbb{O}_{\mathbf{d}} ;\right.
\end{aligned}
$$

and for $j>1$, define $\left(m_{i j}^{d}\right)$ as in (4.15) and (4.16). Then the matrices $\left(m_{i j}^{d}\right)$ clearly verify $(\mathbf{Y d} .2)$. Set $\mathbf{s g n}_{\mathcal{O}_{X}}:=\left(\left(m_{i j}^{d_{1}}\right), \cdots,\left(m_{i j}^{d_{s}}\right)\right)$. It now follows from the last paragraph of Remark 5.21 that $\operatorname{sgn}_{\mathcal{O}_{X}} \in \mathcal{S}_{\mathbf{d}}^{\text {even }}(p, q)$. Thus we have the map

$$
\Psi_{\mathrm{Sp}(p, q)}: \mathcal{N}(\operatorname{Sp}(p, q)) \longrightarrow \mathcal{Y}^{\mathrm{even}}(p, q), \mathcal{O}_{X} \longmapsto\left(\Psi_{\mathrm{Sp}(p, q)}^{\prime}\left(\mathcal{O}_{X}\right), \mathbf{s g n}_{\mathcal{O}_{X}}\right)
$$

where $\mathcal{Y}^{\text {even }}(p, q)$ is as in $(2.10)$.

Theorem 4.38. The above map $\Psi_{\mathrm{Sp}(p, q)}$ is a bijection.

Remark 4.39. On account of the error in [CoMc, Lemma 9.3.1] (mentioned in Remark 5.21), the above parametrization in Theorem 4.38 is a modification of the one given in [CoMc, Theorem 9.3.5].

Theorem 4.40. Let $X \in \mathfrak{s p}(p, q)$ be a nilpotent element. Let $\left(\mathbf{d}, \operatorname{sgn}_{\mathcal{O}_{X}}\right) \in \mathcal{Y}^{\text {even }}(p, q)$ be the signed Young diagram of the orbit $\mathcal{O}_{X}$ (that is, $\Psi_{\operatorname{Sp}(p, q)}\left(\mathcal{O}_{X}\right)=\left(\mathbf{d}, \mathbf{s g n}_{\mathcal{O}_{X}}\right)$ in the notation of Theorem 4.38). Then $\operatorname{dim}_{\mathbb{R}} H^{2}\left(\mathcal{O}_{X}, \mathbb{R}\right)=\# \mathbb{E}_{\mathbf{d}}$.

Proof. As the theorem is evident for $X=0$, we assume that $X \neq 0$.

Let $\{X, H, Y\} \subset \mathfrak{s p}(p, q)$ be a $\mathfrak{s l}_{2}(\mathbb{R})$-triple. Let $V:=\mathbb{H}^{n}$ be the right $\mathbb{H}$-vector space of column vectors. We consider $V$ as a $\operatorname{Span}_{\mathbb{R}}\{X, H, Y\}$-module via its natural $\mathfrak{s p}(p, q)$-module structure. Let

$$
V_{\mathbb{E}}:=\bigoplus_{\eta \in \mathbb{E}_{\mathbf{d}}} M(\eta-1) ; \quad V_{\mathbb{O}}:=\bigoplus_{\theta \in \mathbb{O}_{\mathbf{d}}} M(\theta-1) .
$$

Using Lemma 5.12 we see that $V=V_{\mathbb{E}} \oplus V_{\mathbb{O}}$ is an orthogonal decomposition of $V$ with respect to $\langle\cdot, \cdot\rangle$. Let $\langle\cdot, \cdot\rangle_{\mathbb{E}}:=\left.\langle\cdot, \cdot\rangle\right|_{V_{\mathbb{E}} \times V_{\mathbb{E}}}$ and $\langle\cdot, \cdot\rangle_{\mathbb{O}}:=\left.\langle\cdot, \cdot\rangle\right|_{V_{\mathbb{O}} \times V_{\mathbb{O}}}$. Let $X_{\mathbb{E}}:=\left.X\right|_{V_{\mathbb{E}}}, X_{\mathbb{O}}:=\left.X\right|_{V_{\mathbb{O}}}$, $H_{\mathbb{E}}:=\left.H\right|_{V_{\mathbb{E}}}, H_{\mathbb{O}}:=\left.H\right|_{V_{\mathbb{O}}}, Y_{\mathbb{E}}:=\left.Y\right|_{V_{\mathbb{E}}}$ and $Y_{\mathbb{O}}:=\left.Y\right|_{V_{\mathbb{O}}}$. Then we have the following natural isomorphism:

$$
\mathcal{Z}_{\mathrm{Sp}(p, q)}(X, H, Y) \simeq \mathcal{Z}_{\mathrm{SU}\left(V_{\mathbb{E}},\langle\cdot, \cdot\rangle_{\mathbb{E}}\right)}\left(X_{\mathbb{E}}, H_{\mathbb{E}}, Y_{\mathbb{E}}\right) \times \mathcal{Z}_{\mathrm{SU}\left(V_{\mathbb{O}},\langle\cdot, \cdot\rangle_{\mathbb{O}}\right)}\left(X_{\mathbb{O}}, H_{\mathbb{O}}, Y_{\mathbb{O}}\right)
$$

Recall that the non-degenerate form $(\cdot, \cdot)_{d}$ on $L(d-1)$ is skew-Hermitian for all $d \in \mathbb{E}_{\mathbf{d}}$ and Hermitian for all $d \in \mathbb{O}_{\mathbf{d}}$; see Remark 5.16. Moreover, for any $\theta \in \mathbb{O}_{\mathbf{d}}$, the signature of $(\cdot, \cdot)_{\theta}$ is $\left(p_{\theta}, q_{\theta}\right)$. It follows from Lemma $4.4(4)$ that

$$
\mathcal{Z}_{\mathrm{SU}\left(V_{\mathbb{E}},\langle\cdot, \cdot\rangle_{\mathbb{E}}\right)}\left(X_{\mathbb{E}}, H_{\mathbb{E}}, Y_{\mathbb{E}}\right) \simeq \prod_{\eta \in \mathbb{E}_{\mathbf{d}}} \mathrm{SO}^{*}\left(2 t_{\eta}\right) \text { and } \mathcal{Z}_{\mathrm{SU}\left(V_{\mathbb{O}},\langle\cdot, \cdot\rangle_{\mathbb{O}}\right)}\left(X_{\mathbb{O}}, H_{\mathbb{O}}, Y_{\mathbb{O}}\right) \simeq \prod_{\theta \in \mathbb{O}_{\mathbf{d}}} \operatorname{Sp}\left(p_{\theta}, q_{\theta}\right)
$$

In particular, $\mathcal{Z}_{\mathrm{SU}\left(V_{\mathbb{E}},\langle\cdot, \cdot\rangle_{\mathbb{E}}\right)}\left(X_{\mathbb{E}}, H_{\mathbb{E}}, Y_{\mathbb{E}}\right)$ and $\mathcal{Z}_{\mathrm{SU}\left(V_{\mathbb{O}},\langle\cdot, \cdot\rangle_{\mathbb{O}}\right)}\left(X_{\mathbb{O}}, H_{\mathbb{O}}, Y_{\mathbb{O}}\right)$ are both connected groups. Let $K_{\mathbb{E}}$ be a maximal compact subgroup of $\mathcal{Z}_{\mathrm{SU}\left(V_{\mathbb{E}},\langle\cdot, \cdot\rangle_{\mathbb{E}}\right)}\left(X_{\mathbb{E}}, H_{\mathbb{E}}, Y_{\mathbb{E}}\right) \simeq \prod_{\eta \in \mathbb{E}_{\mathrm{d}}} \mathrm{SO}^{*}\left(2 t_{\eta}\right)$, and let 
$K_{\mathbb{O}}$ be a maximal compact subgroup of $\mathcal{Z}_{\mathrm{SU}\left(V_{\mathbb{O}},\langle\cdot, \cdot\rangle_{\mathbb{O}}\right)}\left(X_{\mathbb{O}}, H_{\mathbb{O}}, Y_{\mathbb{O}}\right) \simeq \prod_{\theta \in \mathbb{O}_{\mathbf{d}}} \operatorname{Sp}\left(p_{\theta}, q_{\theta}\right)$. Let $K$ be the image of $K_{\mathbb{E}} \times K_{\mathbb{O}}$ under the isomorphism as in (4.65). It is clear that $K$ is a maximal compact subgroup of $\mathcal{Z}_{\mathrm{Sp}(p, q)}(X, H, Y)$. Let $M$ be a maximal compact subgroup of $\operatorname{Sp}(p, q)$ containing $K$. As $M \simeq \mathrm{Sp}(p) \times \mathrm{Sp}(q)$ is semisimple, and $K$ is connected, using Theorem 4.2 we have

$$
H^{2}\left(\mathcal{O}_{X}, \mathbb{R}\right) \simeq \mathfrak{z}(\mathfrak{k}), \quad \text { for all } X \neq 0
$$

Let $\mathfrak{k}_{\mathbb{O}}$ and $\mathfrak{k}_{\mathbb{E}}$ be the Lie algebras of $K_{\mathbb{O}}$ and $K_{\mathbb{E}}$ respectively. As $K_{\mathbb{O}}$ is semisimple, we have $\mathfrak{z}\left(\mathfrak{k}_{\mathbb{O}}\right)=0$. Hence, $\mathfrak{z}(\mathfrak{k}) \simeq \mathfrak{z}\left(\mathfrak{k}_{\mathbb{E}}\right) \oplus \mathfrak{z}\left(\mathfrak{k}_{\mathbb{O}}\right)=\mathfrak{z}\left(\mathfrak{k}_{\mathbb{E}}\right)$. Since $\mathfrak{k}_{\mathbb{E}} \simeq \bigoplus_{\eta \in \mathbb{E}_{\mathfrak{d}}} \mathfrak{u}\left(t_{\eta}\right)$, we have $\operatorname{dim}_{\mathbb{R}} \mathfrak{z}\left(\mathfrak{k}_{\mathbb{E}}\right)=$ $\# \mathbb{E}_{\mathbf{d}}$. This completes the proof.

\section{First COHOMOlOGY Groups of NilPotent orbits}

In this section we apply the results of the previous section to compute the first cohomology groups of the nilpotent orbits in the classical simple Lie algebras. We begin by showing that in the case of complex simple Lie algebras the first cohomology groups of all the nilpotent orbits vanish.

Theorem 5.1. Let $\mathfrak{g}$ be a complex simple Lie algebra. Then $H^{1}\left(\mathcal{O}_{X}, \mathbb{R}\right)=0$ for every nilpotent element $X \in \mathfrak{g}$.

Proof. Any maximal compact subgroup of a simple complex Lie Group is simple. The theorem follows from Corollary 3.8.

Theorem 5.2. Let $\mathfrak{g}$ be either $\mathfrak{s l}_{n}(\mathbb{H})$ or $\mathfrak{s p}(p, q)$. Then $H^{1}\left(\mathcal{O}_{X}, \mathbb{R}\right)=0$ for every nilpotent element $X \in \mathfrak{g}$.

Proof. Let $G$ be $\mathrm{SL}_{n}(\mathbb{H})$ or $\operatorname{Sp}(p, q)$ according as $\mathfrak{g}$ is $\mathfrak{s l}_{n}(\mathbb{H})$ or $\mathfrak{s p}(p, q)$. Then any maximal compact subgroup of $G$ is simple. The theorem now follows from Theorem 4.2.

Theorem 5.3. Let $X \in \mathfrak{s l}_{n}(\mathbb{R})$ be a non-zero nilpotent element. Then

$$
\operatorname{dim}_{\mathbb{R}} H^{1}\left(\mathcal{O}_{X}, \mathbb{R}\right)= \begin{cases}1 & \text { if } n=2 \\ 0 & \text { if } n \geq 3 .\end{cases}
$$

Proof. We follow the notation of the proof of Theorem 4.7. When $n \geq 3$, it is clear that $\mathfrak{m}=[\mathfrak{m}, \mathfrak{m}]$. When $n=2$, we have $\mathfrak{m} \simeq \mathfrak{s o}_{2}$ and $\Psi_{\mathrm{SL}_{n}(\mathbb{R})}\left(\mathcal{O}_{X}\right)=\left[2^{1}\right]$. Thus, using (4.9) we see that $\mathfrak{k}=0$. Now the theorem follows from Theorem 4.2 .

Theorem 5.4. Let $X \in \mathfrak{s u}(p, q)$ be a nilpotent element. Let $\left(\mathbf{d}, \mathbf{s g n}_{\mathcal{O}_{X}}\right) \in \mathcal{Y}(p, q)$ be the signed Young diagram of the orbit $\mathcal{O}_{X}$ (that is, $\Psi_{\mathrm{SU}(p, q)}\left(\mathcal{O}_{X}\right)=\left(\mathbf{d}, \mathbf{s g n}_{\mathcal{O}_{X}}\right)$ in the notation of Theorem 4.10). Let $l:=\#\left\{d \in \mathbb{N}_{\mathbf{d}} \mid p_{d} \neq 0\right\}+\#\left\{d \in \mathbb{N}_{\mathbf{d}} \mid q_{d} \neq 0\right\}$. Then the following hold:

(1) If $\mathbb{N}_{\mathbf{d}}=\mathbb{E}_{\mathbf{d}}$, then $\operatorname{dim}_{\mathbb{R}} H^{1}\left(\mathcal{O}_{X}, \mathbb{R}\right)=1$.

(2) If $l=1$ and $\mathbb{N}_{\mathbf{d}}=\mathbb{O}_{\mathbf{d}}$, then $\operatorname{dim}_{\mathbb{R}} H^{1}\left(\mathcal{O}_{X}, \mathbb{R}\right)=1$.

(3) If $l \geq 2$ and $\# \mathbb{O}_{\mathbf{d}} \geq 1$, then $\operatorname{dim}_{\mathbb{R}} H^{1}\left(\mathcal{O}_{X}, \mathbb{R}\right)=0$.

Proof. We follow the notation of the proof of Theorem 4.15. We now appeal to Proposition 4.14 to make the following observations:

(1) If $\mathbb{N}_{\mathbf{d}}=\mathbb{E}_{\mathbf{d}}$, then $\mathfrak{k} \subset[\mathfrak{m}, \mathfrak{m}]$. Hence, $\mathfrak{k}+[\mathfrak{m}, \mathfrak{m}] \varsubsetneqq \mathfrak{m}$.

(2) If $\mathbf{d}=\left[d^{t_{d}}\right]$, then $\mathfrak{z}(\mathfrak{k})=0$. Hence, $\mathfrak{k}+[\mathfrak{m}, \mathfrak{m}] \varsubsetneqq \mathfrak{m}$.

(3) If $\# \mathbb{O}_{\mathbf{d}} \geq 1$ and $l \geq 2$, then $\mathfrak{k}+[\mathfrak{m}, \mathfrak{m}]=\mathfrak{m}$.

As $\operatorname{dim}_{\mathbb{R}} \mathfrak{z}(\mathfrak{m})=1$, the theorem follows from Theorem 4.2. 
We next describe the first cohomology groups of nilpotent orbits in the simple Lie algebra $\mathfrak{s o}(p, q)$ when $p>0, q>0$. Recall that in view of [Kn, Theorem $6.105, \mathrm{p} .421]$ and isomorphisms (iv), (v), (vi), (ix), (x) in [He, Chapter X, §6, pp. 519-520], to ensure simplicity of $\mathfrak{s o}(p, q)$, we further assume that $(p, q) \notin\{(1,1),(2,2)\}$; see $\S 4.5$ also.

Theorem 5.5. Consider $\mathfrak{s o}(p, q)$, and assume that $p \neq 2, q \neq 2$ and $(p, q) \neq(1,1)$. Then $H^{1}\left(\mathcal{O}_{X}, \mathbb{R}\right)=0$ for all nilpotent elements $X$ in $\mathfrak{s o}(p, q)$.

Proof. Let $\mathfrak{m}, \mathfrak{k}$ be as in the proof of Theorem 4.25. Since $p \neq 2, q \neq 2$, we have $\mathfrak{m}=[\mathfrak{m}, \mathfrak{m}]$. Using Theorem 4.2 we conclude that $H^{1}\left(\mathcal{O}_{X}, \mathbb{R}\right)=0$.

We will now consider the remaining cases of $\mathfrak{s o}(p, q)$ which are not covered in Theorem 5.5; they are: $p>2, q=2 ; p=2, q>2$ and $(p, q) \in\{(2,1),(1,2)\}$. In Section 4.5 it was observed that when $p>2, q=2$, the non-zero nilpotent orbits correspond to only four possible signed Young diagrams as given in (a.1), (a.2), (a.3), (a.4), and similarly, when $p=2, q>2$, the non-zero nilpotent orbits correspond to only four possible signed Young diagrams as given in (b.1), (b.2), (b.3), (b.4).

Theorem 5.6. Let $\Psi_{\mathrm{SO}(p, q)^{\circ}}$ be the parametrization in Theorem 4.16. Let $\mathcal{O}_{X} \in \mathcal{N}\left(\mathrm{SO}(p, q)^{\circ}\right)$. Then the following hold:

(1) Suppose $(p, q) \in\{(2,1),(1,2)\}$, then $H^{1}\left(\mathcal{O}_{X}, \mathbb{R}\right)=1$.

(2) Assume that $p>2$ and $q=2$.

(i) If $\Psi_{\mathrm{SO}(p, 2)^{\circ}}\left(\mathcal{O}_{X}\right)$ is as in either (a.1) or (a.2) or $(\mathbf{a} .3)$, then $\operatorname{dim}_{\mathbb{R}} H^{1}\left(\mathcal{O}_{X}, \mathbb{R}\right)=1$.

(ii) If $\Psi_{\mathrm{SO}(p, 2)^{\circ}}\left(\mathcal{O}_{X}\right)$ is as in (a.4), then $H^{1}\left(\mathcal{O}_{X}, \mathbb{R}\right)=0$.

(3) Assume that $p=2$ and $q>2$.

(i) If $\Psi_{\mathrm{SO}(2, q)^{\circ}}\left(\mathcal{O}_{X}\right)$ is as in (b.1) or (b.2) or (b.3), then $\operatorname{dim}_{\mathbb{R}} H^{1}\left(\mathcal{O}_{X}, \mathbb{R}\right)=1$.

(ii) If $\Psi_{\mathrm{SO}(2, q)^{\circ}}\left(\mathcal{O}_{X}\right)$ is as in (b.4), then $H^{1}\left(\mathcal{O}_{X}, \mathbb{R}\right)=0$.

Proof. As $X \neq 0$, it lies in a $\mathfrak{s l}_{2}(\mathbb{R})$-triple, say $\{X, H, Y\}$, in $\mathfrak{s o}(p, q)$.

Proof of (1): Let $K^{\prime}$ be a maximal compact subgroup of $\mathcal{Z}_{\mathrm{SO}(p, q)^{\circ}}(X, H, Y)$. Let $\mathfrak{k}^{\prime}$ be the Lie algebra of $K^{\prime}$ and $\mathfrak{m}$ the Lie algebra of a maximal compact $\operatorname{subgroup~of~} \mathrm{SO}(p, q)^{\circ}$ which contains $K^{\prime}$. When $(p, q) \in\{(2,1),(1,2)\}$, we have $\operatorname{dim}_{\mathbb{R}} \mathfrak{m}=1$ and $\Psi_{\mathrm{SO}(p, q)^{\circ}}^{\prime}\left(\mathcal{O}_{X}\right)=\left[3^{1}\right]$. In particular, $\operatorname{dim}_{\mathbb{R}} L(3-1)=1$. Using Lemma 4.4 (4) we have $\mathfrak{k}^{\prime}=0$. Hence, using Theorem 4.2, we have $\operatorname{dim}_{\mathbb{R}} H^{1}\left(\mathcal{O}_{X}, \mathbb{R}\right)=1$.

Proof of (2): We first prove (2)(i). Let $\Psi_{\mathrm{SO}(p, 2)^{\circ}}\left(\mathcal{O}_{X}\right)$ be as in (a.1), (a.2) or (a.3). Let $K$ and $M$ be the maximal compact subgroups of $\mathcal{Z}_{\mathrm{SO}(p, 2)}(X, H, Y)$ and $\mathrm{SO}(p, 2)$ respectively, as defined in the first paragraph of the proof of Theorem 4.26(2). Recall that $K_{\mathbb{O}}:=K \cap M^{\circ}=$ $K \cap \mathrm{SO}(p, 2)^{\circ}$ is a maximal compact subgroup of $\mathcal{Z}_{\mathrm{SO}(p, 2)^{\circ}}(X, H, Y)$. Let $\mathfrak{k}_{\mathbb{O}}$ and $\mathfrak{m}$ be the Lie algebras of $K_{\mathbb{O}}$ and $M^{\circ}$ respectively. Using (4.32), (4.33), (4.34) for the signed Young diagrams (a.1), (a.2), (a.3) respectively, we observe that in all the cases $\mathfrak{k}_{\mathbb{O}} \subset[\mathfrak{m}, \mathfrak{m}]$. Now (2)(i) follows from Theorem 4.2 .

We next prove (2)(ii). Let $\widetilde{\mathfrak{k}}$ and $\mathfrak{m}$ be as in the proof of (2)(iv) of Theorem 4.26. Then using (4.35), we have $\widetilde{\mathfrak{k}}+[\mathfrak{m}, \mathfrak{m}]=\mathfrak{m}$. The statement (2)(ii) now follows using Theorem 4.2.

The proofs of (3)(i) and (3)(ii) are similar to those of (2)(i) and (2)(ii) respectively.

As we deal with nilpotent orbits in simple Lie algebras, to ensure simplicity of $\mathfrak{s 0 ^ { * }}(2 n)$, in our next result we further assume that $n \geq 3$; see $\S 4.6$ also.

Theorem 5.7. Let $X \in \mathfrak{s o}^{*}(2 n)$ be a nilpotent element when $n \geq 3$. Let $\left(\mathbf{d}, \mathbf{s g n}_{\mathcal{O}_{X}}\right) \in \mathcal{Y}^{\text {odd }}(n)$ be the signed Young diagram of the orbit $\mathcal{O}_{X}$ (that is, $\Psi_{\mathrm{SO}^{*}(2 n)}\left(\mathcal{O}_{X}\right)=\left(\mathbf{d}, \mathbf{s g n}_{\mathcal{O}_{X}}\right)$ in the 
notation of Theorem 4.27). Then

$$
\operatorname{dim}_{\mathbb{R}} H^{1}\left(\mathcal{O}_{X}, \mathbb{R}\right)= \begin{cases}1 & \text { if } \# \mathbb{O}_{\mathbf{d}}=0 \\ 0 & \text { if } \# \mathbb{O}_{\mathbf{d}} \geq 1\end{cases}
$$

Proof. We follow the notation of the proof of Theorem 4.31. Using Proposition 4.30 we have $\mathfrak{k} \subset\left[\mathfrak{k}_{\mathcal{H}}, \mathfrak{k}_{\mathcal{H}}\right]$ when $\# \mathbb{O}_{\mathbf{d}}=0$, and $\mathfrak{k}+\left[\mathfrak{k}_{\mathcal{H}}, \mathfrak{k}_{\mathcal{H}}\right]=\mathfrak{k}_{\mathcal{H}}$ when $\# \mathbb{O}_{\mathbf{d}} \geq 1$. As $\operatorname{dim}_{\mathbb{R}} \mathfrak{z}\left(\mathfrak{k}_{\mathcal{H}}\right)=1$, the proof is completed by Theorem 4.2.

Theorem 5.8. Let $X \in \mathfrak{s p}(n, \mathbb{R})$ be a nilpotent element. Let $\left(\mathbf{d}, \mathbf{s g n}_{\mathcal{O}_{X}}\right) \in \mathcal{Y}_{-1}^{\text {odd }}(2 n)$ be the signed Young diagram of the orbit $\mathcal{O}_{X}$ (that is, $\Psi_{\mathrm{Sp}(n, \mathbb{R})}\left(\mathcal{O}_{X}\right)=\left(\mathbf{d}, \mathbf{s g n}_{\mathcal{O}_{X}}\right)$ in the notation of Theorem 4.32). Then

$$
\operatorname{dim}_{\mathbb{R}} H^{1}\left(\mathcal{O}_{X}, \mathbb{R}\right)= \begin{cases}1 & \text { if } \# \mathbb{O}_{\mathbf{d}}=0 \\ 0 & \text { if } \# \mathbb{O}_{\mathbf{d}} \geq 1\end{cases}
$$

Proof. We follow the notation of the proof of Theorem 4.37. Using Proposition 4.36, we conclude that $\mathfrak{k} \subset\left[\mathfrak{k}_{\mathcal{H}}, \mathfrak{k}_{\mathcal{H}}\right]$ when $\# \mathbb{O}_{\mathbf{d}}=0$ and $\mathfrak{k}+\left[\mathfrak{k}_{\mathcal{H}}, \mathfrak{k}_{\mathcal{H}}\right]=\mathfrak{k}_{\mathcal{H}}$ when $\# \mathbb{O}_{\mathbf{d}} \geq 1$. As $\operatorname{dim}_{\mathbb{R}} \mathfrak{z}\left(\mathfrak{k}_{\mathcal{H}}\right)=1$, the proof is completed by Theorem 4.2 .

\section{ApPEndix. BASIC RESUlts ON NILPOTENT ORBITS}

This appendix is devoted to working out certain details on the structures of the nilpotent orbits in classical Lie algebras so that they can be applied in our computations in Section 4.

We recall that the structures of the nilpotent orbits in classical Lie algebras over $\mathbb{R}$ or $\mathbb{C}$ are due to Springer and Steinberg and they are obtained in [SpSt, p. 249, 1.6; p. 259, 2.19] using elementary linear algebra. However, when classical Lie algebras over $\mathbb{H}$ are considered, due to non-commutativity of $\mathbb{H}$ the above results do not seem to allow direct extensions. Here, by applying the Jacobson-Morozov Theorem and basics of $\mathfrak{s l}_{2}(\mathbb{R})$-representation theory, we overcome this and moreover, work on Lie algebras involving $\mathbb{R}, \mathbb{C}$ and $\mathbb{H}$ simultaneously. This method is suggested in [Mc, § 3.1-3.3, pp. 174-180] and in [CoMc, § 9.3, p. 139].

Following the above approach we also detect an error in [CoMc, Lemma 9.3.1, p. 139] which we point out in Remark 5.21. This led us to modify the definition of signed Young diagrams as given in [CoMc, p. 140] and choose different signs in the last columns of the associated matrices, as done in $\mathbf{Y d} .2$.

We follow the notation established in Section 2.3. The next lemma is an elementary application of the standard structure theory of irreducible $\mathfrak{s l}_{2}(\mathbb{R})$-modules. Let $\mathbb{D}$ denote either $\mathbb{R}, \mathbb{C}$ or $\mathbb{H}$, as before.

Lemma 5.9. Let $V$ be a right $\mathbb{D}$-vector space, and let $\{X, H, Y\} \subset \mathfrak{s l}(V)$ be a $\mathfrak{s l}_{2}(\mathbb{R})$-triple. Let $d$ be a positive integer such that $M(d-1) \neq 0$. Let $\left\{w_{1}, w_{2}, \cdots, w_{t_{d}}\right\}$ be any $\mathbb{D}$-basis of $L(d-1)$. Then

(1) $X^{d} w_{j}=0$ and $H\left(X^{l} w_{j}\right)=X^{l} w_{j}(2 l+1-d)$ for all $1 \leq j \leq t_{d}$;

(2) the set $\left\{X^{l} w_{j} \mid 1 \leq j \leq t_{d}, \quad 0 \leq l \leq d-1\right\}$ is a $\mathbb{D}$-basis of $M(d-1)$;

(3) the $\mathbb{R}$-Span of $\left\{w_{j}, X w_{j}, \cdots, X^{d-1} w_{j}\right\}$ is an irreducible $\operatorname{Span}_{\mathbb{R}}\{X, H, Y\}$-submodule of $M(d-1)$, and moreover, if $W_{j}$ is the $\mathbb{D}$-Span of $\left\{w_{j}, X w_{j}, \cdots, X^{d-1} w_{j}\right\}$, then

$$
M(d-1)=W_{1} \oplus W_{2} \oplus \cdots \oplus W_{t_{d}}=L(d-1) \oplus X L(d-1) \oplus \cdots \oplus X^{d-1} L(d-1) .
$$

Proof. As $M(d-1), V_{Y, 0}$ and $L(d-1)$ of are $\mathbb{D}$-subspaces of $V$, it suffices to prove the lemma for $\mathbb{D}=\mathbb{R}$. We have the following relations: for $1 \leq j \leq t_{d}$ and $0 \leq l \leq d-1$,

$$
H w_{j}=w_{j}(1-d), \quad H\left(X^{l} w_{j}\right)=w_{j}(2 l+1-d) .
$$


Using induction on $l$, it follows from the relations $[H, X]=2 X,[H, Y]=-2 Y,[X, Y]=H$, that $Y X^{l} v=\left(X^{l-1} v\right) l(d-l)$ for all $v \in L(d-1)$ and $l>0$. This in turn implies that

$$
Y^{l} X^{l} w_{j}=w_{j}(l !)(d-1)(d-2) \cdots(d-l) .
$$

Note that $X^{d} w_{j}=0$ because $d-1$ is the highest weight. From (5.2) it follows that $X^{l} w_{j}$ and $X^{k} w_{i}$ are linearly independent if $l \neq k ; 0 \leq l, k \leq d-1 ; 1 \leq j, i \leq t_{d}$. Furthermore, (5.3) implies that for each $l$ with $0 \leq l<d$, the vectors $\left\{X^{l} w_{j} \mid 1 \leq j \leq t_{d}\right\}$ are $\mathbb{R}$-linearly independent. It is a basic fact that $\operatorname{dim}_{\mathbb{R}} M(d-1)=d \operatorname{dim}_{\mathbb{R}} L(d-1)$. Consequently,

$$
\left\{X^{l} w_{j} \mid 1 \leq j \leq t_{d}, 0 \leq l \leq d-1\right\}
$$

is a $\mathbb{R}$-basis of $M(d-1)$. This proves (2). Part (3) follows immediately from (2).

When $\mathbb{D}=\mathbb{R}$ or $\mathbb{C}$ Proposition 5.10 follows from [SpSt, p. 249, 1.6].

Proposition 5.10. Let $\{X, H, Y\} \subset \mathfrak{s l}(V)$ be a $\mathfrak{s l}_{2}(\mathbb{R})$-triple, where $V$ is a right $\mathbb{D}$-vector space. For all $d \in \mathbb{N}_{\mathbf{d}}$ and for any $\mathbb{D}$-basis of $L(d-1)$, say, $\left\{v_{j}^{d} \mid 1 \leq j \leq t_{d}:=\operatorname{dim}_{\mathbb{D}} L(d-1)\right\}$ the following two hold:

(1) $X^{d} v_{j}^{d}=0$ and $H\left(X^{l} v_{j}^{d}\right)=X^{l} v_{j}^{d}(2 l+1-d)$ for $1 \leq j \leq t_{d}, 0 \leq l \leq d-1, d \in \mathbb{N}_{\mathbf{d}}$.

(2) For all $d \in \mathbb{N}_{\mathbf{d}}$, the set $\left\{X^{l} v_{j}^{d} \mid 1 \leq j \leq t_{d}, 0 \leq l \leq d-1\right\}$ is a $\mathbb{D}$-basis of $M(d-1)$. In particular, $\left\{X^{l} v_{j}^{d} \mid 1 \leq j \leq t_{d}, 0 \leq l \leq d-1, d \in \mathbb{N}_{\mathbf{d}}\right\}$ is a $\mathbb{D}$-basis of $V$.

Proof. This follows from Lemma 5.9 and (4.2).

Henceforth, $\sigma: \mathbb{D} \longrightarrow \mathbb{D}$ will denote either the identity map or $\sigma_{c}$ (defined in Section 2.1) when $\mathbb{D}$ is $\mathbb{C}$ or $\mathbb{H}$. Let $V$ be a right $\mathbb{D}$-vector space, and let $\langle\cdot, \cdot\rangle: V \times V \longrightarrow \mathbb{D}$ be a $\epsilon-\sigma$ Hermitian form. Let $X$ be a non-zero nilpotent element in $\mathfrak{s u}(V,\langle\cdot, \cdot\rangle)$. Using Theorem 2.4, there exists $H, Y \in \mathfrak{s u}(V,\langle\cdot, \cdot\rangle)$ such that $\operatorname{Span}_{\mathbb{R}}\{X, H, Y\}$ is isomorphic to $\mathfrak{s l}_{2}(\mathbb{R})$. Thus, $V$ becomes a $\operatorname{Span}_{\mathbb{R}}\{X, H, Y\}$-module.

We record the following straightforward but useful fact.

Lemma 5.11 (cf. [Mc, $\S 2.4$, p. 171]). Let $\sigma: \mathbb{D} \longrightarrow \mathbb{D}$ be either the identity map or $\sigma_{c}$ when $\mathbb{D}$ is $\mathbb{C}$ or $\mathbb{H}$. Let $\langle\cdot, \cdot\rangle: V \times V \longrightarrow \mathbb{D}$ be a $\epsilon-\sigma$ Hermitian form. Suppose $A \in \operatorname{End}_{\mathbb{D}}(V)$ such that $\langle A x, y\rangle+\langle x, A y\rangle=0$ for all $x, y \in V$. Let $v$ and $w$ be two nonzero elements in $V$ such that $A v=v \lambda$ and $A w=w \mu$ for some $\lambda, \mu \in \mathbb{R}$. If $\lambda+\mu \neq 0$, then $\langle v, w\rangle=0$.

Proof. As $\langle A v, w\rangle+\langle v, A w\rangle=0$, it follows immediately that $\langle v, w\rangle(\lambda+\mu)=0$. Now the lemma follows because $\lambda+\mu \neq 0$.

Lemma 5.12 (cf. [Mc, $\S 3.2$, p. 178]). Let $V$ be a right $\mathbb{D}$-vector space, and $\epsilon= \pm 1$. Let $\sigma$ be as in Lemma 5.11, and let

$$
\langle\cdot, \cdot\rangle: V \times V \longrightarrow \mathbb{D}
$$

be a $\epsilon-\sigma$ Hermitian form. Let $\{X, H, Y\} \subset \mathfrak{s u}(V,\langle\cdot, \cdot\rangle)$ be $a \mathfrak{s l}_{2}(\mathbb{R})$-triple. Let $\mathbf{d}$ be as in (4.1), and let $d, d^{\prime} \in \mathbb{N}_{\mathbf{d}}$ be such that $d \neq d^{\prime}$. Then $M(d-1)$ and $M\left(d^{\prime}-1\right)$ are orthogonal with respect to $\langle\cdot, \cdot\rangle$. In particular, the Hermitian form $\langle\cdot, \cdot\rangle$ on $M(d-1)$ is non-degenerate for all $d$.

Proof. We may assume that $d>d^{\prime}$. Let $v \in L(d-1)$ and $u \in L\left(d^{\prime}-1\right)$. By Lemma 5.11 we have that $\left\langle v, X^{l} u\right\rangle=0$ when $0 \leq l \leq d^{\prime}-1$. Moreover, $X^{l} u=0$ if $l \geq d^{\prime}$. Thus $\left\langle v, X^{l} u\right\rangle=0$ for all $l \geq 0$. Hence, $\left\langle X^{h} v, X^{l} u\right\rangle=(-1)^{h}\left\langle v, X^{l+h} u\right\rangle=0$. Now the lemma follows from (5.1) of Lemma 5.9. 
The following lemma, which further decomposes each isotypical component $M(d-1) \subset V$ into orthogonal subspaces, seems basic. However, as we are unable to locate it in the literature, we include a proof here.

Lemma 5.13. Let $V$ be a right $\mathbb{D}$-vector space, and $\epsilon= \pm 1$. Let $\sigma: \mathbb{D} \longrightarrow \mathbb{D}$ be either the identity map or $\sigma_{c}$ when $\mathbb{D}$ is $\mathbb{C}$ or $\mathbb{H}$. Let $\langle\cdot, \cdot\rangle: V \times V \longrightarrow \mathbb{D}$ be a (non-degenerate) $\epsilon-\sigma$ Hermitian form. Let $\{X, H, Y\} \subset \mathfrak{s u}(V,\langle\cdot, \cdot\rangle)$ be a $\mathfrak{s l}_{2}(\mathbb{R})$-triple. Let $\mathbf{d}$ be as in $(4.1), d \in \mathbb{N}_{\mathbf{d}}$ and $t_{d}:=\operatorname{dim}_{\mathbb{D}} L(d-1)$. Then there exists a $\mathbb{D}$-basis $\left\{w_{1}, \cdots, w_{t_{d}}\right\}$ of $L(d-1)$ such that the set

$$
\left\{X^{l} w_{j} \mid 1 \leq j \leq t_{d}, 0 \leq l \leq d-1\right\}
$$

is a $\mathbb{D}$-basis of $M(d-1)$, and moreover, the value of $\langle\cdot, \cdot\rangle$ on a pair of these basis vector is 0 , except in the following cases:

(1) If $\sigma=\sigma_{c}$, then $\left\langle X^{l} w_{j}, X^{d-1-l} w_{j}\right\rangle \in \mathbb{D}^{*}$.

(2) If $\sigma=$ Id and $\epsilon=1$, then $\left\langle X^{l} w_{j}, X^{d-1-l} w_{j}\right\rangle \in \mathbb{D}^{*}$ for $d$ odd, and

$$
\left\langle X^{l} w_{j}, X^{d-1-l} w_{j+1}\right\rangle \in \mathbb{D}^{*}
$$

for $d$ even and $j$ odd.

(3) If $\sigma=\operatorname{Id}$ and $\epsilon=-1$, then $\left\langle X^{l} w_{j}, X^{d-1-l} w_{j}\right\rangle \in \mathbb{D}^{*}$ for $d$ even, and

$$
\left\langle X^{l} w_{j}, X^{d-1-l} w_{j+1}\right\rangle \in \mathbb{D}^{*}
$$

for $d$ odd and $j$ odd.

Proof. We use induction on $\operatorname{dim}_{\mathbb{D}} V$. The proof is divided into two parts.

Part 1. In this part assume that one of the following two holds:

- $\mathbb{D}=\mathbb{R}, \sigma=\mathrm{Id}$, and $(-1)^{d-1} \epsilon=1$;

- $\mathbb{D}=\mathbb{H}$ or $\mathbb{C}$ and $\sigma=\sigma_{c}$.

We claim that there is an element $x_{1} \in L(d-1)$ such that $\left\langle x_{1}, X^{d-1} x_{1}\right\rangle \neq 0$.

To prove the claim by contradiction, assume that $\left\langle x, X^{d-1} x\right\rangle=0$ for all $x \in L(d-1)$. Lemma 5.11 implies that $\left\langle z_{1}, X^{l} z_{2}\right\rangle=0$ for $l \neq d-1$ and $z_{1}, z_{2} \in L(d-1)$. Fix a nonzero element $x \in L(d-1)$. Since $\langle\cdot, \cdot\rangle$ is non-degenerate on $M(d-1) \times M(d-1)$, there exists an element $y \in L(d-1)$ such that $\left\langle x, X^{d-1} y\right\rangle \neq 0$. As $x+y \in L(d-1)$, we also know that $\left\langle x+y, X^{d-1}(x+y)\right\rangle=0$.

Now we will arrive at a contradiction considering the two cases separately.

First assume that $\mathbb{D}=\mathbb{R}, \sigma=\operatorname{Id}$ and $(-1)^{d-1} \epsilon=1$. Then

$$
0=\left\langle x+y, \quad X^{d-1}(x+y)\right\rangle=2\left\langle x, X^{d-1} y\right\rangle .
$$

This is evidently a contradiction.

Next assume that $\mathbb{D}=\mathbb{C}$. Writing $\left\langle x, X^{d-1} y\right\rangle=a_{1}+\sqrt{-1} b_{1}$ where $a_{1}, b_{1} \in \mathbb{R}$ and multiplying $y$ by an appropriate scalar from $\mathbb{C}$ if required, we may assume that $a_{1} \neq 0$ as well as $b_{1} \neq 0$. Now the condition $\left\langle x+y, X^{d-1}(x+y)\right\rangle=0$ implies that $\left(a_{1}+\sqrt{-1} b_{1}\right)+(-1)^{d-1} \epsilon\left(a_{1}-\sqrt{-1} b_{1}\right)=$ 0 . This contradicts the fact that both $a_{1}$ and $b_{1}$ are non-zero.

Finally, assume that $\mathbb{D}=\mathbb{H}$. Writing $\left\langle x, X^{d-1} y\right\rangle=a_{1}+\mathbf{i} b_{1}+\mathbf{j} c_{1}+\mathbf{k} d_{1}$ where $a_{1}, b_{1}, c_{1}, d_{1} \in \mathbb{R}$ and multiplying $y$ by an appropriate scalar from $\mathbb{H}$ if needed, we may assume that $a_{1} \neq 0$ and $b_{1} \neq 0$. Then,

$$
\left\langle x, X^{d-1} y\right\rangle+(-1)^{d-1} \epsilon \sigma\left(\left\langle x, X^{d-1} y\right\rangle\right)=0 .
$$

From this it follows that $\left(a_{1}+\mathbf{i} b_{1}+\mathbf{j} c_{1}+\mathbf{k} d_{1}\right)+(-1)^{d-1} \epsilon\left(a_{1}-\mathbf{i} b_{1}-\mathbf{j} c_{1}-\mathbf{k} d_{1}\right)=0$. This gives a contradiction as both $a_{1}$ and $b_{1}$ are nonzero. This completes the proof of the claim. 
Let $W$ be the $\mathbb{D}$-Span of $\left\{X^{l} x_{1} \mid 0 \leq l \leq d-1\right\}$, where $x_{1}$ is the element of $L(d-1)$ in the above claim. As the vectors $\left\{X^{l} x_{1} \mid 0 \leq l \leq d-1\right\}$ are $\mathbb{D}$-linearly independent, and $\left\langle x_{1}, X^{d-1} x_{1}\right\rangle \neq 0$, it follows that $\langle\cdot, \cdot\rangle$ is non-degenerate on $W$. Hence,

$$
V=W \oplus W^{\perp},
$$

where $W^{\perp}:=\{v \in V \mid\langle v, W\rangle=0\}$. As $\{X, H, Y\} \subset \mathfrak{s u}(V,\langle\cdot, \cdot\rangle)$, it follows immediately that $X, H, Y$ leave $W^{\perp}$ invariant. Let

$$
X_{1}:=\left.X\right|_{W^{\perp}}, \quad H_{1}:=\left.H\right|_{W^{\perp}}, \quad Y_{1}:=\left.Y\right|_{W^{\perp}} .
$$

Let $\langle\cdot, \cdot\rangle^{\prime}$ be the restriction of $\langle\cdot, \cdot\rangle$ to $W^{\perp}$. Then

$$
\left\{X_{1}, H_{1}, Y_{1}\right\} \subset \mathfrak{s u}\left(W^{\perp},\langle\cdot, \cdot\rangle^{\prime}\right)
$$

is a $\mathfrak{s l}_{2}(\mathbb{R})$-triple. Let $M_{W^{\perp}}(d-1)$ be the isotypical component of $W^{\perp}$ consisting of sum of all $\mathbb{R}$ subspaces $B$ of $W^{\perp}$ with $\operatorname{dim}_{\mathbb{R}} B=d$ which are also irreducible $\operatorname{Span}_{\mathbb{R}}\left\{X_{1}, H_{1}, Y_{1}\right\}$-submodules of $W^{\perp}$. Then we have $M_{W \perp}(d-1)=W^{\perp} \cap M(d-1)$ and $M(d-1)=W \oplus M_{W \perp}(d-1)$. Since $\operatorname{dim}_{\mathbb{D}} W^{\perp}<\operatorname{dim}_{\mathbb{D}} V$, from the induction hypothesis, $M_{W^{\perp}}(d-1)$ has a $\mathbb{D}$-basis satisfying (1), (2), (3) of the lemma. This $\mathbb{D}$-basis of $M_{W^{\perp}}(d-1)$ together with the $\mathbb{D}$-basis

$$
\left\{X^{l} x_{1} \mid 0 \leq l \leq d-1\right\}
$$

of $W$ will give the required $\mathbb{D}$-basis of $M(d-1)$. This completes the proof using induction on $\operatorname{dim}_{\mathbb{D}} V$.

Part 2 : Here we deal with the remaining case where $\mathbb{D}=\mathbb{R}, \sigma=\operatorname{Id}$ and $(-1)^{d-1} \epsilon=-1$.

For all $x \in L(d-1)$, as

$$
\left\langle x, X^{d-1} x\right\rangle=(-1)^{d-1} \epsilon\left\langle x, X^{d-1} x\right\rangle=-\left\langle x, X^{d-1} x\right\rangle,
$$

it is clear that $\left\langle x, X^{d-1} x\right\rangle=0$. Lemma 5.11 gives that

$$
\left\langle z_{1}, X^{l} z_{2}\right\rangle=0
$$

for $l \neq d-1, z_{1}, z_{2} \in L(d-1)$. Fix any nonzero $x_{1} \in L(d-1)$. Since $\langle\cdot, \cdot\rangle$ is non-degenerate on $M(d-1) \times M(d-1)$, there exists $y_{1} \in L(d-1) \backslash x_{1} \mathbb{D}$ such that $\left\langle x_{1}, X^{d-1} y_{1}\right\rangle \neq 0$. Let $W^{\prime}$ be the $\mathbb{D}$-Span of $\left\{X^{l} x_{1}, X^{l} y_{1} \mid 0 \leq l \leq d-1\right\}$. As the vectors $\left\{X^{l} x_{1}, X^{l} y_{1} \mid 0 \leq l \leq d-1\right\}$ are $\mathbb{D}$-linearly independent, and $\left\langle x_{1}, X^{d-1} y_{1}\right\rangle \neq 0$, it follows that $\langle\cdot, \cdot\rangle$ is non-degenerate on $W^{\prime}$. As before, define $W^{\prime \perp}:=\left\{v \in V \mid\left\langle v, W^{\prime}\right\rangle=0\right\}$. As $V=W^{\prime} \oplus W^{\prime \perp}$, and $\operatorname{dim}_{\mathbb{D}} W^{\prime \perp}<\operatorname{dim}_{\mathbb{D}} V$, repeating the argument in part 1 the proof is completed.

The next result is an analogue of Proposition 5.10 in the presence of a $\epsilon-\sigma$ Hermitian form. When $\mathbb{D}=\mathbb{R}$ or $\mathbb{C}$ Proposition 5.14 follows from [SpSt, p. 259, 2.19].

Proposition 5.14. Let $V$ be a right $\mathbb{D}$-vector space, $\epsilon= \pm 1, \sigma: \mathbb{D} \longrightarrow \mathbb{D}$ is either the identity map or it is $\sigma_{c}$ when $\mathbb{D}$ is $\mathbb{C}$ or $\mathbb{H}$. Let $\langle\cdot, \cdot\rangle: V \times V \longrightarrow \mathbb{D}$ be a $\epsilon-\sigma$ Hermitian form. Let $\{X, H, Y\} \subset \mathfrak{s u}(V,\langle\cdot, \cdot\rangle)$ be a $\mathfrak{s l}_{2}(\mathbb{R})$-triple. Let $d \in \mathbb{N}_{\mathbf{d}}$ and $t_{d}:=\operatorname{dim}_{\mathbb{D}} L(d-1)$. Then for all $d \in \mathbb{N}_{\mathbf{d}}$, there exists a $\mathbb{D}$-basis $\left\{v_{j}^{d} \mid 1 \leq j \leq t_{d}\right\}$ of $L(d-1)$ such that the following three hold:

(1) $X^{d} v_{j}^{d}=0$ and $H\left(X^{l} v_{j}^{d}\right)=X^{l} v_{j}^{d}(2 l+1-d)$ for all $1 \leq j \leq t_{d}, 0 \leq l \leq d-1$ and $d \in \mathbb{N}_{\mathbf{d}}$.

(2) For all $d \in \mathbb{N}_{\mathbf{d}}$, the set $\left\{X^{l} v_{j}^{d} \mid 1 \leq j \leq t_{d}, 0 \leq l \leq d-1\right\}$ is a $\mathbb{D}$-basis of $M(d-1)$. In particular,

$$
\left\{X^{l} v_{j}^{d} \mid 1 \leq j \leq t_{d}, 0 \leq l \leq d-1, d \in \mathbb{N}_{\mathbf{d}}\right\}
$$

is a $\mathbb{D}$-basis of $V$. 
(3) The value of $\langle\cdot, \cdot\rangle$ on any pair of the above basis vectors is 0 , except in the following cases:

- If $\sigma=\sigma_{c}$, then $\left\langle X^{l} v_{j}^{d}, X^{d-1-l} v_{j}^{d}\right\rangle \in \mathbb{D}^{*}$.

- If $\sigma=\operatorname{Id}$ and $\epsilon=1$, then $\left\langle X^{l} v_{j}^{d}, X^{d-1-l} v_{j}^{d}\right\rangle \in \mathbb{D}^{*}$ when $d \in \mathbb{O}_{\mathbf{d}}$, and

$$
\left\langle X^{l} v_{j}^{d}, X^{d-1-l} v_{j+1}^{d}\right\rangle \in \mathbb{D}^{*}
$$

when $d \in \mathbb{E}_{\mathbf{d}}$ and $j$ is odd.

- If $\sigma=\operatorname{Id}$ and $\epsilon=-1$, then $\left\langle X^{l} v_{j}^{d}, X^{d-1-l} v_{j}^{d}\right\rangle \in \mathbb{D}^{*}$ when $d \in \mathbb{E}_{\mathbf{d}}$, and

$$
\left\langle X^{l} v_{j}^{d}, X^{d-1-l} v_{j+1}^{d}\right\rangle \in \mathbb{D}^{*}
$$

when $d \in \mathbb{O}_{\mathbf{d}}$ and $j$ is odd.

Proof. Lemma 5.12 gives the orthogonal decomposition $V=\bigoplus_{d \in \mathbb{N}_{\mathbf{d}}} M(d-1)$ with respect to the non-degenerate form $\langle\cdot, \cdot\rangle$ on $V$. The proposition now follows from Lemma 5.13.

Let $(\cdot, \cdot)_{d}$ be the form on $L(d-1)$ as defined in (4.6).

Remark 5.15. Observe that if $\{X, H, Y\}$ is a $\mathfrak{s l}_{2}(\mathbb{R})$-triple in the Lie algebra $\mathfrak{s u}(V,\langle\cdot, \cdot\rangle)$, and $g \in \mathcal{Z}_{\mathrm{GL}(V)}(X, H, Y)$, then $(g x, g y)_{d}=(x, y)_{d}$ for all $x, y \in L(d-1)$ if and only if $\langle g v, g w\rangle=\langle v, w\rangle$ for all $v, w \in M(d-1)$.

Remark 5.16. It is easy to see that when $\langle\cdot, \cdot\rangle$ is Hermitian, then the form $(\cdot, \cdot)_{d}$ is Hermitian (respectively, skew-Hermitian) if $d$ is odd (respectively, even). When $\langle\cdot, \cdot\rangle$ is symmetric, then $(\cdot, \cdot)_{d}$ is symmetric (respectively, symplectic) if $d$ is odd (respectively, even). When $\langle\cdot, \cdot\rangle$ is symplectic, then $(\cdot, \cdot)_{d}$ is symplectic (respectively, symmetric) if $d$ is odd (respectively, even). Lastly, when $\langle\cdot, \cdot\rangle$ is skew-Hermitian, then $(\cdot, \cdot)_{d}$ is skew-Hermitian (respectively, Hermitian) if $d$ is odd (respectively, even).

From Lemma 5.13 it follows that $(\cdot, \cdot)_{d}$ is non-degenerate. The $\mathbb{D}$-basis elements

$$
\left\{v_{j}^{d} \mid 1 \leq j \leq t_{d}\right\}
$$

of $L(d-1)$ in Proposition 5.14 are modified as follows:

(1) If $\mathbb{D}=\mathbb{R}$ and $\epsilon=1$, by suitable rescaling each element of $\left\{v_{j}^{d} \mid 1 \leq j \leq t_{d}\right\}$ we may assume that

- $\left\langle v_{j}^{d}, X^{d-1} v_{j}^{d}\right\rangle= \pm 1$ when $d \in \mathbb{O}_{\mathbf{d}}$, and

- $\left\langle v_{j}^{d}, X^{d-1} v_{j+1}^{d}\right\rangle=1$ when $d \in \mathbb{E}_{\mathbf{d}}$ and $j$ is odd.

In particular, $\left(v_{1}^{d}, \cdots, v_{t_{d}}^{d}\right)$ is an standard orthogonal basis of $L(d-1)$ with respect to $(\cdot, \cdot)_{d}$ for $d \in \mathbb{O}_{\mathbf{d}}$. If $\mathbb{D}=\mathbb{R}$ and $\epsilon=-1$, analogously we may assume that the elements of the $\mathbb{R}$-basis

$$
\left\{v_{j}^{d} \mid 1 \leq j \leq t_{d}\right\}
$$

of $L(d-1)$ in Proposition 5.14 satisfy the condition that

- $\left\langle v_{j}^{d}, X^{d-1} v_{j}^{d}\right\rangle= \pm 1$ when $d \in \mathbb{E}_{\mathbf{d}}$, and

- $\left\langle v_{j}^{d}, X^{d-1} v_{t_{d} / 2+j}^{d}\right\rangle=1$ when $d \in \mathbb{O}_{\mathbf{d}}$ and $1 \leq j \leq t_{d} / 2$.

In particular, $\left(v_{1}^{d}, \cdots, v_{t_{d}}^{d}\right)$ is an orthogonal basis for $d \in \mathbb{E}_{\mathbf{d}}$, and

$$
\left(v_{1}^{d}, \cdots, v_{t_{d} / 2}^{d} ; v_{t_{d} / 2+1}^{d}, \cdots, v_{t_{d}}^{d}\right)
$$

is a symplectic basis for $d \in \mathbb{O}_{\mathbf{d}}$ of $L(d-1)$ with respect to $(\cdot, \cdot)_{d}$.

(2) If $\mathbb{D}=\mathbb{C}, \epsilon=1$ and $\sigma=\sigma_{c}$, rescaling the elements of the $\mathbb{C}$-basis $\left\{v_{j}^{d} \mid 1 \leq j \leq t_{d}\right\}$ we may assume that

- $\left\langle v_{j}^{d}, X^{d-1} v_{j}^{d}\right\rangle= \pm 1$ when $d \in \mathbb{O}_{\mathbf{d}}$, and

- $\left\langle v_{j}^{d}, X^{d-1} v_{j}^{d}\right\rangle= \pm \sqrt{-1}$ when $d \in \mathbb{E}_{\mathbf{d}}$. 
In particular, $\left(v_{1}^{d}, \cdots, v_{t_{d}}^{d}\right)$ is an orthogonal basis of $L(d-1)$ with respect to $(\cdot, \cdot)_{d}$ for $d \in \mathbb{N}_{\mathbf{d}}$.

(3) If $\mathbb{D}=\mathbb{H}, \epsilon=1$ and $\sigma=\sigma_{c}$, after rescaling and conjugating the elements of the $\mathbb{H}$-basis $\left\{v_{j}^{d} \mid 1 \leq j \leq t_{d}\right\}$ of $L(d-1)$ by suitable scalars (see Lemma 2.3) the elements of the $\mathbb{H}$-basis, we may assume that

- $\left\langle v_{j}^{d}, X^{d-1} v_{j}^{d}\right\rangle= \pm 1$ when $d \in \mathbb{O}_{\mathbf{d}}$, and

- $\left\langle v_{j}^{d}, X^{d-1} v_{j}^{d}\right\rangle=\mathbf{j}$ when $d \in \mathbb{E}_{\mathbf{d}}$.

If $\mathbb{D}=\mathbb{H}, \epsilon=-1$ and $\sigma=\sigma_{c}$, analogously we may assume that the elements of the $\mathbb{H}$-basis $\left\{v_{j}^{d} \mid 1 \leq j \leq t_{d}\right\}$ of $L(d-1)$ satisfy

- $\left\langle v_{j}^{d}, X^{d-1} v_{j}^{d}\right\rangle= \pm 1$ when $d \in \mathbb{E}_{\mathbf{d}}$, and

- $\left\langle v_{j}^{d}, X^{d-1} v_{j}^{d}\right\rangle=\mathbf{j}$ when $d \in \mathbb{O}_{\mathbf{d}}$.

Let $\left(v_{1}^{d}, \cdots, v_{t_{d}}^{d}\right)$ be an ordered $\mathbb{D}$-basis of $L(d-1)$ as in Proposition 5.14 satisfying the properties as Remark 5.16. The proofs of the following lemmas are straightforward and they are omitted.

Lemma 5.17. Let $\mathbb{D}=\mathbb{R}, \sigma=\operatorname{Id}$ and $\epsilon=1$. Fix $d \in \mathbb{N}_{\mathbf{d}}$ and $1 \leq j \leq t_{d}$.

(1) If $\eta \in \mathbb{E}_{\mathbf{d}}$ and $j$ is odd, define

$$
w_{j l}^{\eta}:= \begin{cases}\left(X^{l} v_{j}^{\eta}+X^{\eta-1-l} v_{j+1}^{\eta}\right) \frac{1}{\sqrt{2}} & \text { if } 0 \leq l \leq \eta-1 \\ \left(X^{2 \eta-1-l} v_{j}^{\eta}-X^{l-\eta} v_{j+1}^{\eta}\right) \frac{1}{\sqrt{2}} & \text { if } \eta \leq l \leq 2 \eta-1 .\end{cases}
$$

Then $\left\langle w_{j l}^{\eta}, w_{j(2 \eta-1-l)}^{\eta}\right\rangle=0$, and $\left\langle w_{j l}^{\eta}, w_{j l}^{\eta}\right\rangle=(-1)^{l}\left\langle v_{j}^{\eta}, X^{\eta-1} v_{j+1}^{\eta}\right\rangle$.

(2) For $\theta \in \mathbb{O}_{\mathbf{d}}$, define

$$
w_{j l}^{\theta}:= \begin{cases}\left(X^{l} v_{j}^{\theta}+X^{\theta-1-l} v_{j}^{\theta}\right) \frac{1}{\sqrt{2}} & \text { if } 0 \leq l<(\theta-1) / 2 \\ X^{l} v_{j}^{\theta} & \text { if } l=(\theta-1) / 2 \\ \left(X^{\theta-1-l} v_{j}^{\theta}-X^{l} v_{j}^{\theta}\right) \frac{1}{\sqrt{2}} & \text { if }(\theta-1) / 2<l \leq \theta-1 .\end{cases}
$$

Then $\left\langle w_{j l}^{\theta}, w_{j(\theta-1-l)}^{\theta}\right\rangle=0$ and

$$
\left\langle w_{j l}^{\theta}, w_{j l}^{\theta}\right\rangle= \begin{cases}(-1)^{l}\left\langle v_{j}^{\theta}, X^{\theta-1} v_{j}^{\theta}\right\rangle & \text { if } 0 \leq l<(\theta-1) / 2 \\ (-1)^{l}\left\langle v_{j}^{\theta}, X^{\theta-1} v_{j}^{\theta}\right\rangle & \text { if } l=(\theta-1) / 2 \\ (-1)^{l+1}\left\langle v_{j}^{\theta}, X^{\theta-1} v_{j}^{\theta}\right\rangle & \text { if }(\theta-1) / 2<l \leq \theta-1 .\end{cases}
$$

Therefore, for any $\theta \in \mathbb{O}_{\mathbf{d}}$,

$$
\left\langle w_{j l}^{\theta}, w_{\left.j l^{\prime}\right)}^{\theta}\right\rangle=0
$$

when $l \neq l^{\prime}$ and $0 \leq l, l^{\prime} \theta-1$.

Lemma 5.18. Let $\mathbb{D}=\mathbb{C}, \sigma=\sigma_{c}$ and $\epsilon=1$. Fix $d \in \mathbb{N}_{\mathbf{d}}$ and $1 \leq j \leq t_{d}$.

(1) For $\eta \in \mathbb{E}_{\mathbf{d}}$, define

$$
\widetilde{w}_{j l}^{\eta}:= \begin{cases}\left(X^{l} v_{j}^{\eta}+X^{\eta-1-l} v_{j}^{\eta} \sqrt{-1}\right) \frac{1}{\sqrt{2}} & \text { if } 0 \leq l<\eta / 2 \\ \left(X^{\eta-1-l} v_{j}^{\eta}-X^{l} v_{j}^{\eta} \sqrt{-1}\right) \frac{1}{\sqrt{2}} & \text { if } \eta / 2 \leq l \leq \eta-1 .\end{cases}
$$

Then $\left\langle\widetilde{w}_{j l}^{\eta}, \widetilde{w}_{j(\eta-1-l)}^{\eta}\right\rangle=0$ and $\left\langle\widetilde{w}_{j l}^{\eta}, \widetilde{w}_{j l}^{\eta}\right\rangle=(-1)^{l} \sqrt{-1}\left\langle v_{j}^{\eta}, X^{\eta-1} v_{j}^{\eta}\right\rangle$. 
(2) For $\theta \in \mathbb{O}_{\mathbf{d}}$, define

$$
\widetilde{w}_{j l}^{\theta}:= \begin{cases}\left(X^{l} v_{j}^{\theta}+X^{\theta-1-l} v_{j}^{\theta}\right) \frac{1}{\sqrt{2}} & \text { if } 0 \leq l<(\theta-1) / 2 \\ X^{l} v_{j}^{\theta} & \text { if } l=(\theta-1) / 2 \\ \left(X^{\theta-1-l} v_{j}^{\theta}-X^{l} v_{j}^{\theta}\right) \frac{1}{\sqrt{2}} & \text { if }(\theta-1) / 2<l \leq \theta-1 .\end{cases}
$$

Then $\left\langle\widetilde{w}_{j l}^{\theta}, \widetilde{w}_{j(\theta-1-l)}^{\theta}\right\rangle=0$, and

$$
\left\langle\widetilde{w}_{j l}^{\theta}, \widetilde{w}_{j l}^{\theta}\right\rangle= \begin{cases}(-1)^{l}\left\langle v_{j}^{\theta}, X^{\theta-1} v_{j}^{\theta}\right\rangle & \text { if } 0 \leq l<(\theta-1) / 2 \\ (-1)^{l}\left\langle v_{j}^{\theta}, X^{\theta-1} v_{j}^{\theta}\right\rangle & \text { if } l=(\theta-1) / 2 \\ (-1)^{l+1}\left\langle v_{j}^{\theta}, X^{\theta-1} v_{j}^{\theta}\right\rangle & \text { if }(\theta-1) / 2<l \leq \theta-1 .\end{cases}
$$

Therefore, for any $\theta \in \mathbb{O}_{\mathbf{d}}$,

$$
\left\langle\widetilde{w}_{j l}^{\theta}, \widetilde{w}_{\left.j l^{\prime}\right)}^{\theta}\right\rangle=0
$$

when $l \neq l^{\prime}$ and $0 \leq l, l^{\prime} \leq \theta-1$.

Lemma 5.19. Let $\mathbb{D}=\mathbb{H}, \sigma=\sigma_{c}$ and $\epsilon=1$. Fix $d$ and $1 \leq j \leq t_{d}$.

(1) For $\eta \in \mathbb{E}_{\mathbf{d}}$, define

$$
\widehat{w}_{j l}^{\eta}:= \begin{cases}\left(X^{l} v_{j}^{\eta}+X^{\eta-1-l} v_{j}^{\eta} \alpha_{j}\right) \frac{1}{\sqrt{2}} & \text { if } 0 \leq l<\eta / 2 \\ \left(X^{\eta-1-l} v_{j}^{\eta}-X^{l} v_{j}^{\eta} \alpha_{j}\right) \frac{1}{\sqrt{2}} & \text { if } \eta / 2 \leq l \leq \eta-1\end{cases}
$$

where $\alpha_{j}=\left\langle v_{j}^{\eta}, X^{\eta-1} v_{j}^{\eta}\right\rangle$. Then

$$
\left\langle\widehat{w}_{j l}^{\eta}, \widehat{w}_{j(\eta-1-l)}^{\eta}\right\rangle=0 \quad \text { and } \quad\left\langle\widehat{w}_{j l}^{\eta}, \widehat{w}_{j l}^{\eta}\right\rangle=(-1)^{l+1} \operatorname{Nrd}\left(\left\langle v_{j}^{\eta}, X^{\eta-1} v_{j}^{\eta}\right\rangle\right) .
$$

(2) When $\theta \in \mathbb{O}_{\mathbf{d}}$, define

$$
\widehat{w}_{j l}^{\theta}:= \begin{cases}\left(X^{l} v_{j}^{\theta}+X^{\theta-1-l} v_{j}^{\theta}\right) \frac{1}{\sqrt{2}} & \text { if } 0 \leq l<(\theta-1) / 2 \\ X^{l} v_{j}^{\theta} & \text { if } l=(\theta-1) / 2 \\ \left(X^{\theta-1-l} v_{j}^{\theta}-X^{l} v_{j}^{\theta}\right) \frac{1}{\sqrt{2}} & \text { if }(\theta-1) / 2<l \leq \theta-1 .\end{cases}
$$

Then $\left\langle\widehat{w}_{j l}^{\theta}, \widehat{w}_{j(\theta-1-l)}^{\theta}\right\rangle=0$, and

$$
\left\langle\widehat{w}_{j l}^{\theta}, \widehat{w}_{j l}^{\theta}\right\rangle= \begin{cases}(-1)^{l}\left\langle v_{j}^{\theta}, X^{\theta-1} v_{j}^{\theta}\right\rangle & \text { if } 0 \leq l<(\theta-1) / 2 \\ (-1)^{l}\left\langle v_{j}^{\theta}, X^{\theta-1} v_{j}^{\theta}\right\rangle & \text { if } l=(\theta-1) / 2 \\ (-1)^{l+1}\left\langle v_{j}^{\theta}, X^{\theta-1} v_{j}^{\theta}\right\rangle & \text { if }(\theta-1) / 2<l \leq \theta-1 .\end{cases}
$$

Therefore, for any $d \in \mathbb{N}_{\mathbf{d}}$,

$$
\left\langle\widehat{w}_{j l}^{d}, \widehat{w}_{j l^{\prime}}^{d}\right\rangle=0
$$

when $l \neq l^{\prime}$ and $0 \leq l, l^{\prime} \leq d-1$.

The next corollary, which closely follows [CoMc, Lemma 9.3.1], gives a direct correspondence between the signature of $(\cdot, \cdot)_{d}$ on $L(d-1)$ and the signature of $\langle\cdot, \cdot\rangle$ on $M(d-1)$ when both $\langle\cdot, \cdot\rangle$ and $(\cdot, \cdot)_{d}$ have signatures. In part $(3)$ of the corollary we record a correct version of a result in [CoMc, Lemma 9.3.1]. Recall that $\mathbb{O}_{\mathbf{d}}^{1}$ and $\mathbb{O}_{\mathbf{d}}^{3}$ are as in (2.4).

Corollary 5.20. Let $\langle\cdot, \cdot\rangle$ be a $\epsilon-\sigma$ Hermitian form on $V$. Assume that $\epsilon=1$, that is, the form $\langle\cdot, \cdot\rangle$ is symmetric or Hermitian. 
(1) If $d \in \mathbb{E}_{\mathbf{d}}$, then the signature of $\langle\cdot, \cdot\rangle$ on $M(d-1)$ is $\left(\operatorname{dim}_{\mathbb{D}} M(d-1) / 2, \operatorname{dim}_{\mathbb{D}} M(d-\right.$ 1) $/ 2)$.

(2) If $d \in \mathbb{O}_{\mathbf{d}}^{1}$, and $\left(p_{d}, q_{d}\right)$ is the signature of $(\cdot, \cdot)_{d}$, then the signature of $\langle\cdot, \cdot\rangle$ on $M(d-1)$ is

$$
\left(\left(\operatorname{dim}_{\mathbb{D}} M(d-1)+p_{d}-q_{d}\right) / 2,\left(\operatorname{dim}_{\mathbb{D}} M(d-1)+q_{d}-p_{d}\right) / 2\right) .
$$

(3) If $d \in \mathbb{O}_{\mathbf{d}}^{3}$, and $\left(p_{d}, q_{d}\right)$ is the signature of $(\cdot, \cdot)_{d}$, then the signature of $\langle\cdot, \cdot\rangle$ on $M(d-1)$ is

$$
\left(\left(\operatorname{dim}_{\mathbb{D}} M(d-1)+q_{d}-p_{d}\right) / 2,\left(\operatorname{dim}_{\mathbb{D}} M(d-1)+p_{d}-q_{d}\right) / 2\right) .
$$

Proof. This follows directly from Lemmas 5.17, 5.18 and 5.19.

Remark 5.21. We will now point out an error in [CoMc, p. 139, Lemma 9.3.1], and also explain why the definition of $m_{i d}^{d}$ in the case of $d \in \mathbb{O}_{\mathbf{d}}^{3}$ as in $\mathbf{Y d} .2$ (in Section 2.4) is different from that in the case of $d \in \mathbb{E}_{\mathbf{d}} \cup \mathbb{O}_{\mathbf{d}}^{1}$. Let $V$ be a right $\mathbb{D}$-vector space, and let $\langle\cdot, \cdot\rangle$ be a Hermitian (respectively symmetric) form on $V$ if $\mathbb{D}=\mathbb{H}, \mathbb{C}$ (respectively, $\mathbb{D}=\mathbb{R}$ ). Take a $\mathfrak{s l}_{2}(\mathbb{R})$-triple $\{X, H, Y\} \subset \mathfrak{s u}(V,\langle\cdot, \cdot\rangle)$. Note that if $d \in \mathbb{O}_{\mathbf{d}}^{3}$, then the form $(\cdot, \cdot)_{d}$ in (4.6) is Hermitian (respectively, symmetric) when $\mathbb{D}=\mathbb{H}, \mathbb{C}$ (respectively, $\mathbb{D}=\mathbb{R}$ ). Let $\left(p_{d}, q_{d}\right)$ be the signature of $(\cdot, \cdot)_{d}$ when $d \in \mathbb{O}_{\mathbf{d}}^{3}$. Corollary $5.20(3)$ says that the signature of $\langle\cdot, \cdot\rangle$ restricted to $M(d-1)$ is

$$
\left(\left(\operatorname{dim}_{\mathbb{D}} M(d-1)+q_{d}-p_{d}\right) / 2,\left(\operatorname{dim}_{\mathbb{D}} M(d-1)+p_{d}-q_{d}\right) / 2\right)
$$

when $d \in \mathbb{O}_{\mathbf{d}}^{3}$. Set the signs in first column of the matrix $\left(m_{i j}^{d}\right)$ as in Yd.1, and thus define $m_{i 1}^{d}=+1$ when $1 \leq i \leq p_{d}$, and define $m_{i 1}^{d}=-1$ when $p_{d}<i \leq t_{d}$.

However, in the case of $d \in \mathbb{O}_{\mathbf{d}}^{3}$, if we, following [CoMc, p. 139, Lemma 9.3.1], define

$$
m_{i j}^{d}=(-1)^{j+1} m_{i 1}^{d}
$$

for $1<j \leq d$, then it can be easily verified that

$$
\left(\operatorname{sgn}_{+}\left(m_{i j}^{d}\right), \operatorname{sgn}_{-}\left(m_{i j}^{d}\right)\right)=\left(\left(\operatorname{dim}_{\mathbb{D}} M(d-1)+p_{d}-q_{d}\right) / 2,\left(\operatorname{dim}_{\mathbb{D}} M(d-1)+q_{d}-p_{d}\right) / 2\right) .
$$

Thus, if $d \in \mathbb{O}_{\mathbf{d}}^{3}$ and $p_{d} \neq q_{d}$, then appealing to Corollary 5.20(3) we see that the signature of $\langle\cdot, \cdot\rangle$ restricted to $M(d-1)$ does not coincide with $\left(\operatorname{sgn}_{+}\left(m_{i j}^{d}\right), \operatorname{sgn}_{-}\left(m_{i j}^{d}\right)\right)$. This shows that the second statement of [CoMc, p. 139, Lemma 9.3.1] is not true when $d \in \mathbb{O}_{\mathbf{d}}^{3}$ and $p_{d} \neq q_{d}$ (this means that $r \equiv 2(\bmod 4)$ in the notation of [CoMc, p. 139, Lemma 9.3.1]). Recall that in Yd.2 (see Section 2.4), when $d \in \mathbb{O}_{\mathbf{d}}^{3}$ we have defined $m_{i j}^{d}=(-1)^{j+1} m_{i 1}^{d}$ when $1<j \leq d-1$ while $m_{i d}^{d}:=-m_{i 1}^{d}$. Using the definitions of $m_{i 1}^{d}$ as above we have that

$$
\left(\operatorname{sgn}_{+}\left(m_{i j}^{d}\right), \operatorname{sgn}_{-}\left(m_{i j}^{d}\right)\right)=\left(\left(\operatorname{dim}_{\mathbb{D}} M(d-1)+q_{d}-p_{d}\right) / 2,\left(\operatorname{dim}_{\mathbb{D}} M(d-1)+p_{d}-q_{d}\right) / 2\right) .
$$

Thus, if we define $m_{i j}^{d}$ as in $\mathbf{Y d} .1$ and $\mathbf{Y d} .2$, then the signature of $\langle\cdot, \cdot\rangle$ on $M(d-1)$ does coincide with $\left(\operatorname{sgn}_{+}\left(m_{i j}^{d}\right), \operatorname{sgn}_{-}\left(m_{i j}^{d}\right)\right)$ for $d \in \mathbb{N}_{\mathbf{d}}$.

\section{REFERENCES}

[Al] A. Alexeevski, Component groups of the centralizers of unipotent elements in semisimple algebraic groups, in: Lie groups and invariant theory, Amer. Math. Soc. Transl. Ser. 2, 213, (Amer. Math. Soc., Providence, RI, 2005), pp. 15-31.

[ABB] H. Azad, E. van den Ban and I. Biswas, Symplectic geometry of semisimple orbits. Indag. Math. 19 (2008), no. 4, 507-533.

[Bo1] A. Borel, Sur la cohomologie des espaces fibrés principaux et des espaces homogènes de groupes de Lie compacts, Ann. of Math. 57 (1953), 115-207.

[Bo2] A. Borel, Linear algebraic groups, Second Enlarged Edition, Graduate Texts in Mathematics, 126, Springer-Verlag, New York, 1991.

[Br] G. Bredon, Topology and geometry, Graduate Texts in Mathematics, 139, Springer-Verlag, New York, 1997. 
[BC1] I. Biswas and P. Chatterjee, On the exactness of Kostant-Kirillov form and the second cohomology of nilpotent orbits, Internat. J. Math. 23 (2012), no. 8, 1250086.

[BC2] I. Biswas and P. Chatterjee, Second cohomology of compact homogeneous spaces, Internat. J. Math. 24 (2013), no. 9, 1350076.

[CE] C. Chevalley and S. Eilenberg, Cohomology theory of Lie groups and Lie algebras, Trans. Amer. Math. Soc. 63 (1948), 85-124.

[ChMa] P. Chatterjee and C. Maity, On the second cohomology of nilpotent orbits in exceptional Lie algebras, Bull. Sci. Math. 141 (2017), 10-24.

[CoMc] D. H. Collingwood and W. M. McGovern, Nilpotent orbits in semisimple Lie algebras, Van Nostrand Reinhold Mathematics Series, Van Nostrand Reinhold Co., New York, 1993.

[Cr] P. Crooks, The torus-equivariant cohomology of nilpotent orbits, J. Lie Theory, 25 (2015), no. 4, 1073-1087.

[GHV] W. Greub, S. Halperin and R. Vanstone, Connections, curvature, and cohomology, Volume III: Cohomology of principal bundles and homogeneous spaces, Pure and Applied Mathematics, Vol. 47-III. Academic Press, New York-London, 1976.

[He] S. Helgason, Differential Geometry, Lie Groups, and Symmetric Spaces, Academic Press, New York, 1978.

[Ho] G. Hochschild, The structure of Lie groups, Holden-Day, Inc., San Francisco, 1965.

[Ju] D. Juteau, Cohomology of the Minimal Nilpotent Orbit, Transformation Groups, Vol. 13, No. 2 (2008), $355-387$.

[Ki] D. King, The Component Groups of Nilpotents in Exceptional Simple Real Lie Algebras, Communications in Algebra, 20(1), (1992), 219-284.

[Kn] A. W. Knapp, Lie groups beyond an introduction, Second edition, Progress in Mathematics, Vol. 140, Birkhäusar, Basel, 2002.

[Mc] W. M. McGovern, The adjoint representation and the adjoint action, in: Algebraic quotients. Torus actions and cohomology. The adjoint representation and the adjoint action, 159-238, Encyclopaedia Math. Sci., 131, Springer, Berlin, 2002.

[Mo] G. D. Mostow, On covariant fiberings of Klein spaces, Amer. Jour. Math. 77 (1955), $247-278$.

[So] E. Sommers, A generalization of the Bala-Carter theorem for nilpotent orbits, Internat. Math. Res. Notices, No. 11, (1998), 539-562.

[Sp] M. Spivak, A comprehensive introduction to differential geometry, Vol. V, Third edition, Publish or Perish, Inc., Houston, Texas, 1999.

[SpSt] T. A. Springer and R. Steinberg, Conjugacy classes, in : 1970 Seminar on Algebraic Groups and Related Finite Groups (The Institute for Advanced Study, Princeton, N.J., 1968/69) pp. 167-266, Lecture Notes in Mathematics, Vol. 131 Springer, Berlin, 1970.

School of Mathematics, Tata Institute of Fundamental Research, Homi Bhabha Road, Mumbai 400005, INDIA

Email address: indranil@math.tifr.res.in

The Institute of Mathematical Sciences, HBni, Cit Campus, Tharamani, Chennai 600113, India

Email address: pralay@imsc.res.in

The Institute of Mathematical Sciences, HBni, Cit Campus, Tharamani, Chennai 600113, India

Email address: cmaity@imsc.res.in 